\title{
Thermal Stability and Explosive Hazard Assessment of Diazo Compounds and Diazo Transfer Reagents
}

\author{
Sebastian P. Green, ${ }^{\dagger, \ddagger}$ Katherine M. Wheelhouse, § Andrew D. Payne,\| Jason P. \\ Hallett, ${ }^{\star, \ddagger}$ Philip W. Miller, ${ }^{*, \dagger}$ James A. Bull ${ }^{*, \dagger}$
}

tDepartment of Chemistry, Imperial College London, Molecular Sciences Research Hub, White City Campus, 80 Wood Lane, London W12 0BZ, U.K.

抽epartment of Chemical Engineering, Imperial College London, South Kensington Campus, Exhibition Road, London SW7 2AZ, U.K.

\|Process Safety, Pilot Plant Operations, GlaxoSmithKline, GSK Medicines Research Centre, Gunnels Wood Road, Stevenage, Hertfordshire SG1 2NY, U.K.

§Global API Chemistry, Product Development \& Supply, GlaxoSmithKline, GSK Medicines Research Centre, Gunnels Wood Road, Stevenage, Hertfordshire SG1 2NY, U.K.

*E-mail: j.hallett@imperial.ac.uk

*E-mail: philip.miller@imperial.ac.uk

*E-mail: j.bull@imperial.ac.uk

General Experimental Considerations.................................................................. S2

Synthesis Experimental Procedures and Characterisation ............................................. S3

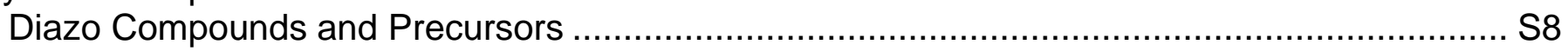

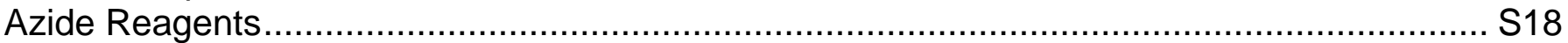

Hammett $\sigma$ Parameters Correlated with DSC Results (Table S1) ................................. S20

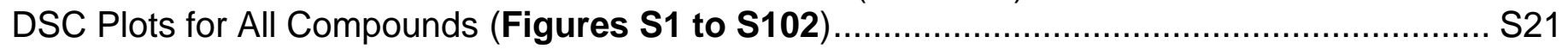

Abnormalities Observed in DSC plots of Diazo Compounds (Figure S103) .......................... S75

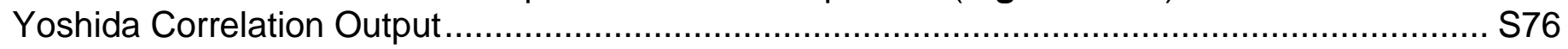

Yoshida IS and EP Correlations for Literature Diazo Transfer Reagent Data (Table S2) ....... S76

Yoshida IS and EP Correlations for All Diazo Compound DSC Data (Table S3) ................... S77

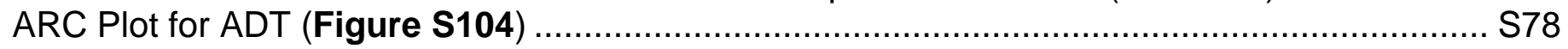

Impact Sensitivity of Well-Defined Explosives (Table S4) ........................................ S79

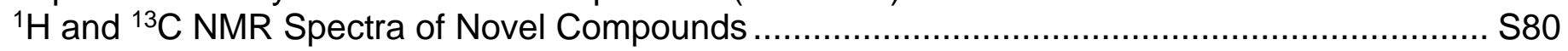

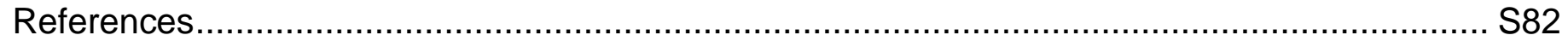

Notes:

- All raw and processed data for this manuscript can be found at the Imperial College London Research

Data Repository (https://doi.org/10.14469/hpc/6274)

- Although we have not experienced any problems in the handling of azides or diazo reagents,

care should be taken when manipulating them due to their potentially explosive nature. 


\section{General Experimental Considerations}

Experimental considerations for the DSC methods used can be found in the Experimental Section of the manuscript.

All non-aqueous reactions were carried out under an inert atmosphere (nitrogen or argon) with flame-dried glassware, using standard techniques. Anhydrous solvents were obtained by filtration through drying columns $\left(\mathrm{CH}_{2} \mathrm{Cl}_{2}, \mathrm{EtOH}, \mathrm{MeCN}\right.$, toluene) or used as supplied (methanol). Flash chromatography was performed using 230-400 mesh silica with the indicated solvent system according to standard techniques. Analytical thin-layer chromatography (TLC) was performed on precoated glass-backed silica gel plates. Visualization of the developed chromatogram was performed by UV absorbance $(254 \mathrm{~nm})$ and stained with aqueous potassium permanganate solution if required.

Infrared spectra ( $v_{\max }$, FTIR ATR) were recorded in reciprocal centimetres $\left(\mathrm{cm}^{-1}\right)$.

Nuclear magnetic resonance spectra were recorded on $400 \mathrm{MHz}$ spectrometers. The frequency used to record the NMR spectra is given in each assignment and spectrum $\left({ }^{1} \mathrm{H} \mathrm{NMR}\right.$ at $400 \mathrm{MHz} ;{ }^{13} \mathrm{C} \mathrm{NMR}$ at $101 \mathrm{MHz} ;{ }^{19} \mathrm{~F}$ NMR at $377 \mathrm{MHz}$ ). Chemical shifts for ${ }^{1} \mathrm{H}$ NMR spectra are recorded in parts per million with the residual protic solvent resonance as the internal standard $\left(\mathrm{CDCl}_{3}: \delta=7.26 \mathrm{ppm}, \mathrm{MeOD}-d_{4}\right.$ : $\delta=3.35 \mathrm{ppm}, \mathrm{C}_{6} \mathrm{D}_{6}: \delta=7.16 \mathrm{ppm}$ ). Data is reported as follows: chemical shift (multiplicity $[\mathrm{s}=$ singlet, $\mathrm{d}=$ doublet, $\mathrm{t}=$ triplet, $\mathrm{q}=$ quartet, $\mathrm{p}=$ pentet, $\mathrm{m}=$ multiplet and $\mathrm{br}=$ broad], integration, coupling constant (in $\mathrm{Hz}$ ), and assignment). ${ }^{13} \mathrm{C}$ NMR spectra were recorded with complete proton decoupling. Chemical shifts are reported in parts per million with the residual protic solvent resonance as the internal standard $\left(\mathrm{CDCl}_{3}\right.$ : $\delta=77.2 \mathrm{ppm}$, MeOD- $d_{4}: \delta=49.0 \mathrm{ppm}, \mathrm{C}_{6} \mathrm{D}_{6}: \delta=128.1 \mathrm{ppm}$ ). Assignments of ${ }^{1} \mathrm{H}$ and ${ }^{13} \mathrm{C}$ spectra were based upon the analysis of $\delta$ and $J$ values. ${ }^{19} \mathrm{~F}$ NMR spectra were recorded with complete proton decoupling. ${ }^{19} \mathrm{~F}$ NMR spectra are indirectly referenced to $\mathrm{CFCl}_{3}$ automatically via direct measurement of the absolute frequency of the deuterium lock signal by the spectrometer hardware. For clarity NMR spectra are displayed as follows unless this would obscure signals: ${ }^{1} \mathrm{H}$ NMR spectra are displayed between $10 \mathrm{ppm}$ and $-0.2 \mathrm{ppm} ;{ }^{13} \mathrm{C}$ NMR spectra are displayed between $210 \mathrm{ppm}$ and $0 \mathrm{ppm} ;{ }^{19} \mathrm{~F}$ NMR spectra displayed for the full sweep width as acquired.

For ${ }^{13} \mathrm{C}$ NMR of the diazo compounds, the resonance for the fully substituted diazo-bearing carbon atom is often not observed due to quadrupole coupling to ${ }^{14} \mathrm{~N}$, in which cases it is not reported.

Melting points were not obtained for diazo compounds or azides, unless there was a clear melting point observed in the DSC thermogram, when this is reported, in which case the temperature of the endothermic peak ( $\left.T_{\text {peak }}\right)$ is rounded to the nearest ${ }^{\circ} \mathrm{C}$.

The high resolution mass spectrometry (HRMS) analyses were performed using electrospray ion source $(\mathrm{ESI}+)$ or pneumatically assisted electrospray $(\mathrm{pNSI})$. ESI+ was performed using a Waters LCT Premier equipped with an ESI source operated in positive ion mode. The software used was MassLynx 4.1, this software does not account for the electron and all the calibrations/references are calculated accordingly, i.e. $[\mathrm{M}+\mathrm{H}]+$ is detected and the mass is calibrated to output $[\mathrm{M}+\mathrm{H}]$. pNSI was performed using an Orbitrap XL in positive ion mode. Samples are loop injected into or infused in a stream of $\mathrm{H}_{2} \mathrm{O} / \mathrm{CH}_{3} \mathrm{OH}\left(1: 1\right.$ at $\left.50 \mu \mathrm{L} \mathrm{min}{ }^{-1}\right)$ using an appropriate solvent for dissolution of the sample. Nebulization was pneumatically assisted by a flow of $\mathrm{N}_{2}$ through a sheath around the capillary. 


\section{Synthetic Experimental Procedures and Characterisation}

The following reagents were commercially available and were used as received:

Ethyl (phenyl)acetate was purchased from Sigma Aldrich (product code:108049). ADMP was purchased from Tokyo Chemical Industry UK Ltd (product code: A2457). p-ABSA was purchased from Tokyo Chemical Industry UK Ltd (product code: A1786) or Fluorochem Ltd (product code: 450489). The DSC and ARC data for $p$-ABSA was obtained from the TCl sample. Ethyl diazoacetate (EDA) was purchased from Sigma Aldrich as a solution containing $13 \%$ weight $\mathrm{CH}_{2} \mathrm{Cl}_{2}$ (product code: E22201) and used as received.

\section{Preparation of alkyl arylacetates (S1-S9):}

\section{tert-Butyl (phenyl)acetate (S1)}<smiles>CCCCOC(=O)Cc1ccccc1</smiles>

DMAP (0.49 g, $4.0 \mathrm{mmol}, 0.8$ equiv) and phenylacetic acid $(0.68 \mathrm{~g}, 5.0 \mathrm{mmol}, 1.0$ equiv) were added to a stirred solution of DCC $\left(1.13 \mathrm{~g}, 5.5 \mathrm{mmol}, 1.1\right.$ equiv) in $\mathrm{CH}_{2} \mathrm{Cl}_{2}(27.3 \mathrm{~mL})$ at $0{ }^{\circ} \mathrm{C}$. tert-Butanol $(1.5 \mathrm{~mL}$, $15 \mathrm{mmol}, 3.0$ equiv) was then slowly added before the mixture was then warmed to rt and stirred for $18 \mathrm{~h}$. The mixture was concentrated in vacuo, diluted in $\mathrm{Et}_{2} \mathrm{O}(30 \mathrm{~mL})$ and filtered through Celite to remove a yellow-white precipitate. The filtrate was washed with $1 \mathrm{M} \mathrm{aq} \mathrm{NaOH}(25 \mathrm{~mL}), 1 \mathrm{M}$ aq HCl $(25 \mathrm{~mL})$ and brine $(25 \mathrm{~mL})$, dried over sodium sulfate and concentrated in vacuo. The residue was then purified by column chromatography using $5 \%$ EtOAc in hexane affording $\mathbf{S 1}(0.21 \mathrm{~g}, 22 \%)$ as a colourless liquid. $\mathbf{R}_{f}=0.48(5 \%$ EtOAc in hexane). IR (ATR)/cm ${ }^{-1}$ 2978, 2931, 1729 (C=O), 1455, 1367, 1254, 1137, 953, 743, 695. ${ }^{1} \mathrm{H}$ NMR $\left(400 \mathrm{MHz}, \mathrm{CDCl}_{3}\right) \delta 7.34-7.23(\mathrm{~m}, 5 \mathrm{H}, 5 \times \mathrm{Ar}-\mathrm{H}), 3.54\left(\mathrm{~s}, 2 \mathrm{H}, \mathrm{CH}_{2}\right), 1.44\left(\mathrm{~s}, 9 \mathrm{H}, 3 \times \mathrm{CH}_{3}\right) .{ }^{13} \mathrm{C} \mathrm{NMR}(101$ $\left.\mathrm{MHz}, \mathrm{CDCl}_{3}\right) \delta 171.1(\mathrm{C}=\mathrm{O}), 134.8\left(\mathrm{Ar} \mathrm{C}_{\mathrm{q}}\right), 129.3(2 \times \mathrm{Ar} \mathrm{CH}), 128.6(2 \times \mathrm{Ar} \mathrm{CH}), 126.9(\mathrm{Ar} \mathrm{CH}), 80.9(\mathrm{tBu}$ $\left.\mathrm{C}_{\mathrm{q}}\right), 42.8\left(\mathrm{CH}_{2}\right), 28.2\left(3 \times \mathrm{CH}_{3}\right)$. The observed characterisation data (IR, ${ }^{1} \mathrm{H}$ and ${ }^{13} \mathrm{C}$ NMR) was consistent with that previously reported in the literature. ${ }^{1,2}$

SMILES: $\mathrm{O}=\mathrm{C}(\mathrm{OC}(\mathrm{C})(\mathrm{C}) \mathrm{C}) \mathrm{CC} 1=\mathrm{CC}=\mathrm{CC}=\mathrm{C} 1$

InChI = 1S/C12H16O2/c1-12(2,3)14-11(13)9-10-7-5-4-6-8-10/h4-8H,9H2,1-3H3

(1R,2S,5R)-2-Isopropyl-5-methylcyclohexyl 2-phenylacetate (S2)<smiles>CC1CC[C@H](OC(=O)Cc2ccccc2)C(C(C)C)C1</smiles>

DMAP (1.30 g, $10.7 \mathrm{mmol}, 0.8$ equiv), phenylacetic acid ( $1.82 \mathrm{~g}, 13.3 \mathrm{mmol}, 1.0$ equiv) then (-)-menthol $(6.25 \mathrm{~g}, 40 \mathrm{mmol}, 3.0$ equiv) were added to a stirred solution of DCC (3.03 g, $14.7 \mathrm{mmol}, 1.1$ equiv) in $\mathrm{CH}_{2} \mathrm{Cl}_{2}(73 \mathrm{~mL})$ at $0{ }^{\circ} \mathrm{C}$. The resulting mixture was stirred at $\mathrm{rt}$ for $20 \mathrm{~h}$. The mixture was concentrated in vacuo, diluted with $\mathrm{Et}_{2} \mathrm{O}(100 \mathrm{~mL})$ and filtered through Celite. The filtrate was washed with $1 \mathrm{M}$ aq $\mathrm{NaOH}$ $(50 \mathrm{~mL}), 1 \mathrm{M}$ aq HCl $(50 \mathrm{~mL})$, brine $(50 \mathrm{~mL})$, dried over sodium sulfate and concentrated in vacuo. Purification by column chromatography using 5\% EtOAc in hexane afforded $\mathbf{S 2}(2.92 \mathrm{~g}, 80 \%)$ as a colourless oil. $R_{f}=0.39$ (5\% EtOAc in hexane). IR (film)/cm-1 3033, 2953, 2927, 2870, $1728(\mathrm{C}=0), 1604$, 1497, 1454, 1369, 1255, 1219, 1145, 1097, 759, 721, 695. ${ }^{1} \mathrm{H}$ NMR $\left(400 \mathrm{MHz}, \mathrm{CDCl}_{3}\right) \delta$ 7.37-7.26 (m, 5H, $5 \times \mathrm{Ar}-\mathrm{H}), 4.71(\mathrm{td}, 1 \mathrm{H}, J=11.2,4.0 \mathrm{~Hz}, \mathrm{OCH}), 3.63\left(\mathrm{~s}, 2 \mathrm{H}, \mathrm{PhCH}_{2}\right), 2.03-1.94\left(\mathrm{~m}, 1 \mathrm{H},(\mathrm{Me})_{2} \mathrm{CH}\right)$, 1.80-1.74 (m, $1 \mathrm{H}, \mathrm{MeCH}), 1.73-1.65\left(\mathrm{~m}, 2 \mathrm{H}, \mathrm{OCHCH}_{2}\right), 1.54-1.33\left(\mathrm{~m}, 2 \mathrm{H}, \mathrm{PrCHCH}_{2}\right), 1.11-1.01(\mathrm{~m}, 1 \mathrm{H}$, IPrCH), $1.00-0.89\left(\mathrm{~m}, 2 \mathrm{H}, \mathrm{MeCHCH}_{2}\right), 0.94-0.86\left(\mathrm{~m}, 6 \mathrm{H}, J=6.8 \mathrm{~Hz},\left(\mathrm{CH}_{3}\right)_{2} \mathrm{CH}\right), 0.72(\mathrm{~d}, 3 \mathrm{H}, J=6.8 \mathrm{~Hz}$, $\left.\mathrm{CHCH}_{3}\right) .{ }^{13} \mathrm{C} \operatorname{NMR}\left(101 \mathrm{MHz}, \mathrm{CDCl}_{3}\right) \delta 171.2(\mathrm{C}=\mathrm{O}), 134.4\left(\operatorname{Ar~} \mathrm{C}_{q}\right), 129.2(2 \times \mathrm{Ar} \mathrm{CH}), 128.5(2 \times \mathrm{Ar} \mathrm{CH})$, 
$126.9(\mathrm{Ar} \mathrm{CH}), 74.7(\mathrm{OCH}), 47.1(\mathrm{PrCH}), 41.9\left(\mathrm{OCHCH}_{2}\right), 40.8\left(\mathrm{PhCH}_{2}\right), 34.3\left(\mathrm{MeCHCH}_{2}\right), 31.4(\mathrm{MeCH})$, $26.1\left(\mathrm{CH}(\mathrm{Me})_{2}\right), 23.4\left(\mathrm{CH}_{2} \mathrm{CH}(\mathrm{Pr})\right), 22.0\left(\mathrm{CH}\left(\mathrm{CH}_{3}\right) \mathrm{CH}_{3}\right), 20.7\left(\mathrm{CH}\left(\mathrm{CH}_{3}\right) \mathbf{C H}_{3}\right), 16.3\left(\mathrm{CH}\left(\mathrm{CH}_{3}\right)\right)$. HRMS (pNSI) $\mathrm{m} / \mathrm{z}$ calculated for $\mathrm{C}_{18} \mathrm{H}_{30} \mathrm{NO}_{2}{ }^{+}\left[\mathrm{M}+\mathrm{NH}_{4}\right]^{+}:$292.2271. Found: 292.2269. The observed characterisation data $\left(\mathrm{IR},{ }^{1} \mathrm{H}\right.$ and ${ }^{13} \mathrm{C} \mathrm{NMR}$ ) was consistent with that previously reported in the literature. ${ }^{3}$

SMILES: O=C(O[C@H]1[C@H](C(C)C)CC[C@@H](C)C1)CC2=CC=CC=C2

$\mathrm{InChI}=1 \mathrm{~S} / \mathrm{C} 18 \mathrm{H} 26 \mathrm{O} 2 / \mathrm{c} 1-13(2) 16-10-9-14(3) 11-17(16) 20-18(19) 12-15-7-5-4-6-8-15 / \mathrm{h} 4-8,13-14,16-17 \mathrm{H}, 9-$ $12 \mathrm{H} 2,1-3 \mathrm{H} 3 / \mathrm{t} 14-, 16+, 17-/ \mathrm{m} 1 / \mathrm{s} 1$

\section{2,2,2-Trichloroethyl (phenyl)acetate (S3)}<smiles>O=C(Cc1ccccc1)OCC(Cl)(Cl)Cl</smiles>

DCC (3.40 g, $16.5 \mathrm{mmol}, 1.1$ equiv) dissolve in $\mathrm{CH}_{2} \mathrm{Cl}_{2}$ was added to a solution of phenylacetic acid (2.04 g, $15.0 \mathrm{mmol}, 1.0$ equiv) and PTSA (380 mg, $2.0 \mathrm{mmol}, 0.1$ equiv). After stirring for $24 \mathrm{~h}$, the mixture was filtered, extracted with $\mathrm{Et}_{2} \mathrm{O}(20 \mathrm{ml})$ and concentrated in vacuo. Purification by column chromatography using $2 \% \mathrm{Et}_{2} \mathrm{O}$ in hexane afforded $\mathrm{S} 3(3.56 \mathrm{~g}, 89 \%)$ as a colourless liquid. $\mathrm{R}_{f}=0.18$ ( $2 \% \mathrm{Et}_{2} \mathrm{O}$ in pentane). IR (ATR)/cm ${ }^{-1} 1752$ (C=O), 1498, 1454, 1372, 1230, 1126, 1029, 798, 712. ${ }^{1} \mathrm{H} \mathrm{NMR}\left(400 \mathrm{MHz}, \mathrm{CDCl}_{3}\right) \delta$ 7.37-7.27 (m, 5H, Ar-H), $4.75\left(\mathrm{~s}, 2 \mathrm{H}, \mathrm{OCH}_{2}\right), 3.77\left(\mathrm{~s}, 2 \mathrm{H}, \mathrm{PhCH}_{2}\right) .{ }^{13} \mathrm{C} \mathrm{NMR}\left(101 \mathrm{MHz}, \mathrm{CDCl}_{3}\right) \delta 170.1$ $(\mathrm{C}=\mathrm{O}), 133.1\left(\mathrm{Ar} \mathrm{C}_{\mathrm{q}}\right), 129.6(2 \times \mathrm{Ar} \mathrm{CH}), 128.8(2 \times \mathrm{Ar} \mathrm{CH}), 127.6(\mathrm{Ar} \mathrm{CH}), 95.0\left(\mathrm{CCl}_{3}\right), 74.3\left(\mathrm{CH}_{2}\right), 41.1$ $\left(\mathrm{OCH}_{2}\right)$. The observed characterisation data $\left(\mathrm{IR},{ }^{1} \mathrm{H}\right.$ and $\left.{ }^{13} \mathrm{C} \mathrm{NMR}\right)$ was consistent with that previously reported in the literature..$^{4,5}$

SMILES: $\mathrm{O}=\mathrm{C}(\mathrm{OCC}(\mathrm{Cl})(\mathrm{Cl}) \mathrm{Cl}) \mathrm{CC} 1=\mathrm{CC}=\mathrm{CC}=\mathrm{C} 1$

InChl=1S/C10H9Cl3O2/c11-10(12,13)7-15-9(14)6-8-4-2-1-3-5-8/h1-5H,6-7H2

\section{Ethyl (3-methylphenyl)acetate (S4)}<smiles>CCOC(=O)Cc1cccc(C)c1</smiles>

A stirred solution of (3-methylphenyl)acetic acid (3.00 g, $20 \mathrm{mmol}, 1.0$ equiv) and PTSA (380 mg, $2.0 \mathrm{mmol}$, 0.1 equiv) in ethanol $(50 \mathrm{~mL})$ was heated under reflux for $18 \mathrm{~h}$. After cooling, the ethanol was evaporated in vacuo and saturated aq $\mathrm{NaHCO}_{3}(30 \mathrm{~mL})$ was added. The mixture was extracted with $\mathrm{Et}_{2} \mathrm{O}(3 \times 30 \mathrm{~mL})$, then the organic phases were combined, washed with brine $(30 \mathrm{~mL})$, dried over sodium sulfate and filtered. The filtrate was concentrated in vacuo afforded $\mathbf{S 4}(3.39 \mathrm{~g}, 95 \%)$ as a colourless liquid. $\mathrm{R}_{f}=0.44\left(3 \% \mathrm{Et}_{2} \mathrm{O}\right.$ in pentane). IR (ATR)/cm ${ }^{-1}$ 2981, $1732(\mathrm{C}=\mathrm{O}), 1609,1491,1446,1367,1254,1138,1031 .{ }^{1} \mathrm{H}$ NMR (400 $\left.\mathrm{MHz}_{\mathrm{CDCl}}\right) \delta 7.21(\mathrm{t}, 1 \mathrm{H}, J=7.3 \mathrm{~Hz}, \mathrm{Ar}-\mathrm{H}), 7.10-7.07(\mathrm{~m}, 3 \mathrm{H}, 3 \times \mathrm{Ar}-\mathrm{H}), 4.15(\mathrm{q}, 2 \mathrm{H}, J=7.1 \mathrm{~Hz}, 2 \mathrm{H}$, $\left.\mathrm{OCH}_{2}\right), 3.57\left(\mathrm{~s}, 2 \mathrm{H}, \mathrm{CH}_{2}\right), 2.34\left(\mathrm{~s}, 3 \mathrm{H}, \mathrm{Ar}-\mathrm{CH}_{3}\right), 1.25\left(\mathrm{t}, 3 \mathrm{H}, \mathrm{J}=7.1 \mathrm{~Hz}, \mathrm{CH}_{3}\right) .{ }^{13} \mathrm{C} \mathrm{NMR}\left(101 \mathrm{MHz}, \mathrm{CDCl}_{3}\right) \delta$ $171.7(\mathrm{C}=\mathrm{O})$, $138.1\left(\mathrm{Ar} \mathrm{C}_{\mathrm{q}}-\mathrm{CH}_{3}\right), 134.0(\mathrm{Ar} \mathrm{C}$ ), $130.0(\mathrm{Ar} \mathrm{CH}), 128.4(\mathrm{Ar} \mathrm{CH}), 127.7(\mathrm{Ar} \mathrm{CH}), 126.2(\mathrm{Ar} \mathrm{CH})$, $60.7\left(\mathrm{OCH}_{2}\right), 41.3\left(\mathrm{CH}_{2}\right), 21.3\left(\mathrm{Ar}-\mathrm{CH}_{3}\right), 14.1\left(\mathrm{CH}_{3}\right)$. The observed characterisation data $\left({ }^{1} \mathrm{H}\right.$ and ${ }^{13} \mathrm{C}$ NMR) was consistent with that previously reported in the literature. ${ }^{6,7}$

SMILES: $\mathrm{O}=\mathrm{C}(\mathrm{OCC}) \mathrm{CC} 1=\mathrm{CC}(\mathrm{C})=\mathrm{CC}=\mathrm{C} 1$

$\operatorname{lnChl}=1 \mathrm{~S} / \mathrm{C} 11 \mathrm{H} 14 \mathrm{O} 2 / \mathrm{c} 1-3-13-11(12) 8-10-6-4-5-9(2) 7-10 / \mathrm{h} 4-7 \mathrm{H}, 3,8 \mathrm{H} 2,1-2 \mathrm{H} 3$ 


\section{Ethyl (3-trifluoromethylphenyl)acetate (S5)}<smiles>CCOC(=O)Cc1cccc(C(F)(F)F)c1</smiles>

A stirred solution of (3-trifluoromethylphenyl)acetic acid $(2.04 \mathrm{~g}, 10 \mathrm{mmol}, 1.0$ equiv) and PTSA (190 mg, $1.0 \mathrm{mmol}, 0.1$ equiv) in ethanol $(25 \mathrm{~mL})$ was heated under reflux for $24 \mathrm{~h}$. After cooling, the ethanol was evaporated in vacuo and saturated aq $\mathrm{NaHCO}_{3}(20 \mathrm{~mL})$ was added. The mixture was extracted with $\mathrm{Et}_{2} \mathrm{O}$ $(3 \times 10 \mathrm{~mL})$, then the organic phases were combined, washed with brine $(20 \mathrm{~mL})$, dried over sodium sulfate and filtered. The filtrate was concentrated in vacuo afforded S5 $(1.98 \mathrm{~g}, 85 \%)$ as a colourless liquid.

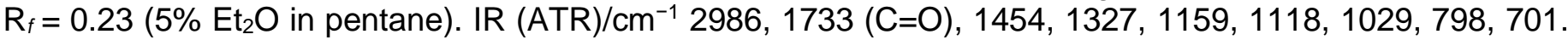
${ }^{1} \mathrm{H}$ NMR $\left(400 \mathrm{MHz}, \mathrm{CDCl}_{3}\right) \delta 7.55-7.43(\mathrm{~m}, 4 \mathrm{H}, 4 \times \mathrm{Ar}-\mathrm{H}), 4.17\left(\mathrm{q}, 2 \mathrm{H}, J=7.2 \mathrm{~Hz}, \mathrm{OCH}_{2}\right), 3.67(\mathrm{~s}, 2 \mathrm{H}$, $\left.\mathrm{CH}_{2}\right), 1.26\left(\mathrm{t}, 3 \mathrm{H}, J=7.2 \mathrm{~Hz}, \mathrm{CH}_{3}\right) .{ }^{13} \mathrm{C} \operatorname{NMR}\left(101 \mathrm{MHz}, \mathrm{CDCl}_{3}\right) \delta 171.0(\mathrm{C}=\mathrm{O}), 135.1\left(\mathrm{Ar} \mathrm{C}_{\mathrm{q}}\right), 132.9(\mathrm{Ar} \mathrm{CH})$, 131.0 (q, $\left.J_{\mathrm{CF}}=30.6 \mathrm{~Hz}, \mathrm{C}_{\mathrm{q}}-\mathrm{CF}_{3}\right), 129.1(\mathrm{ArCH}), 126.3\left(\mathrm{q}, \mathrm{J}_{\mathrm{CF}}=3.7 \mathrm{~Hz}, \mathrm{ArCH}\right), 124.2\left(\mathrm{q}, J_{\mathrm{CF}}=271.2 \mathrm{~Hz}\right.$, $\left.\mathrm{CF}_{3}\right), 124.1$ (q, J $\left.\mathrm{JFF}=3.7 \mathrm{~Hz}, \mathrm{ArCH}\right), 61.3\left(\mathrm{OCH}_{2}\right), 41.2\left(\mathrm{CH}_{2}\right), 14.3\left(\mathrm{CH}_{3}\right) \cdot{ }^{19} \mathrm{~F}\left\{{ }^{1} \mathrm{H}\right\} \mathrm{NMR}\left(377 \mathrm{MHz}, \mathrm{CDCl}_{3}\right) \delta$ -62.7 .

SMILES: $\mathrm{O}=\mathrm{C}(\mathrm{OCC}) \mathrm{CC} 1=\mathrm{CC}(\mathrm{C}(\mathrm{F})(\mathrm{F}) \mathrm{F})=\mathrm{CC}=\mathrm{C} 1$

InChI=1S/C11H11F3O2/c1-2-16-10(15)7-8-4-3-5-9(6-8)11(12,13)14/h3-6H,2,7H2,1H3

\section{Ethyl (2-methoxyphenyl)acetate (S6)}<smiles>CCOC(=O)Cc1ccccc1OC</smiles>

A stirred solution of (2-methoxyphenyl)acetic acid $(3.32 \mathrm{~g}, 20 \mathrm{mmol}, 1.0$ equiv) and PTSA (380 mg, $2.0 \mathrm{mmol}$, 0.1 equiv) in ethanol $(50 \mathrm{~mL})$ was heated under reflux for $18 \mathrm{~h}$. After cooling, the ethanol was evaporated in vacuo and saturated aq $\mathrm{NaHCO}_{3}(30 \mathrm{~mL})$ was added. The mixture was extracted with $\mathrm{Et}_{2} \mathrm{O}(3$ $\times 30 \mathrm{~mL}$ ) and the organic phases were combined, washed with brine $(30 \mathrm{~mL})$, dried over sodium sulfate and filtered. The filtrate was concentrated in vacuo afforded $\mathbf{S 6}(3.28 \mathrm{~g}, 85 \%)$ as a faint yellow liquid. IR (ATR)/cm ${ }^{-1}$ 2981, 1732 (C=O), 1603, 1496, 1464, 1245, 1153, 1113, 1028, 753. ${ }^{1} \mathrm{H} \mathrm{NMR}\left(400 \mathrm{MHz}, \mathrm{CDCl}_{3}\right)$ $\delta 7.26(\mathrm{td}, 1 \mathrm{H}, J=7.7,1.8 \mathrm{~Hz}, \mathrm{Ar}-\mathrm{H}), 7.19(\mathrm{dd}, 1 \mathrm{H}, J=7.5,1.7 \mathrm{~Hz}, \mathrm{Ar}-\mathrm{H}), 6.92(\mathrm{td}, 1 \mathrm{H}, J=7.5,1.0 \mathrm{~Hz}$, $\mathrm{Ar}-\mathrm{H}), 6.87(\mathrm{~d}, 1 \mathrm{H}, J=8.2 \mathrm{~Hz}, \mathrm{Ar}-\mathrm{H}), 4.16\left(\mathrm{q}, 2 \mathrm{H}, J=7.1 \mathrm{~Hz}, \mathrm{OCH}_{2}\right), 3.82(\mathrm{~s}, 3 \mathrm{H}, \mathrm{OMe}), 3.62\left(\mathrm{~s}, 2 \mathrm{H}, \mathrm{CH}_{2}\right)$, 1.25 (t, 3H, $\left.J=7.1 \mathrm{~Hz}, \mathrm{CH}_{3}\right) .{ }^{13} \mathrm{C}$ NMR $\left(101 \mathrm{MHz}, \mathrm{CDCl}_{3}\right) \delta 171.8(\mathrm{C}=\mathrm{O}), 157.5\left(\mathrm{Ar} \mathrm{C}_{\mathrm{q}}-\mathrm{OMe}\right), 130.8(\mathrm{Ar}$ $\mathrm{CH}), 128.4(\mathrm{Ar} \mathrm{CH}), 123.2\left(\mathrm{Ar} \mathrm{C}_{\mathrm{q}}\right), 120.5(\mathrm{Ar} \mathrm{CH}), 110.5(\mathrm{Ar} \mathrm{CH}), 60.5\left(\mathrm{OCH}_{2}\right), 55.4(\mathrm{OMe}), 35.9\left(\mathrm{CH}_{2}\right), 14.2$ $\left(\mathrm{CH}_{3}\right)$. The observed characterisation data (IR, ${ }^{1} \mathrm{H}$ and $\left.{ }^{13} \mathrm{C} \mathrm{NMR}\right)$ was consistent with that previously reported in the literature. ${ }^{8}$

SMILES: $\mathrm{O}=\mathrm{C}(\mathrm{OCC}) \mathrm{CC} 1=\mathrm{C}(\mathrm{OC}) \mathrm{C}=\mathrm{CC}=\mathrm{C} 1$

InChI = 1S/C11H14O3/c1-3-14-11(12)8-9-6-4-5-7-10(9)13-2/h4-7H,3,8H2,1-2H3

\section{Ethyl (2-trifluoromethylphenyl)acetate (S7)}<smiles>CCOC(=O)Cc1ccccc1C(F)(F)F</smiles>

A stirred solution of (2-trifluoromethylphenyl)acetic acid $(2.041 \mathrm{~g}, 10 \mathrm{mmol}, 1.0$ equiv) and PTSA (190 mg, $1.0 \mathrm{mmol}, 0.1$ equiv) in ethanol $(25 \mathrm{~mL})$ was heated under reflux for $24 \mathrm{~h}$. After cooling, the ethanol was evaporated in vacuo and saturated $\mathrm{aq} \mathrm{NaHCO}_{3}(20 \mathrm{~mL})$ was added. The mixture was extracted with $\mathrm{Et}_{2} \mathrm{O}$ $(3 \times 10 \mathrm{~mL})$ and the organic phases were combined, washed with water $(2 \times 10 \mathrm{~mL})$, brine $(10 \mathrm{~mL})$, dried over sodium sulfate and filtered. The filtrate was concentrated in vacuo afforded $\mathbf{S 7}(2.05 \mathrm{~g}, 88 \%)$ as a 
colourless liquid. $\mathrm{R}_{f}=0.12$ (3\% $\mathrm{Et}_{2} \mathrm{O}$ in pentane). IR (ATR)/cm ${ }^{-1}$ 2986, $1737(\mathrm{C}=\mathrm{O}), 1610,1454,1312$, 1215, 1107, 1036, 768. ${ }^{1} \mathrm{H}$ NMR $\left(400 \mathrm{MHz}, \mathrm{CDCl}_{3}\right) \delta 7.66(\mathrm{~d}, 1 \mathrm{H}, \mathrm{J}=7.4 \mathrm{~Hz}, \mathrm{Ar}-\mathrm{H}), 7.52(\mathrm{t}, 1 \mathrm{H}, \mathrm{J}=7.6 \mathrm{~Hz}$, $\mathrm{Ar}-\mathrm{H}), 7.40-7.37(\mathrm{~m}, 2 \mathrm{H}, 2 \times \mathrm{Ar}-\mathrm{H}), 4.17\left(\mathrm{q}, 2 \mathrm{H}, \mathrm{J}=7.1 \mathrm{~Hz}, \mathrm{OCH}_{2}\right), 3.82\left(\mathrm{~s}, 2 \mathrm{H}, \mathrm{CH}_{2}\right), 1.25(\mathrm{t}, 3 \mathrm{H}, \mathrm{J}=7.1$ $\left.\mathrm{Hz}, \mathrm{CH}_{3}\right) .{ }^{13} \mathrm{C}$ NMR $\left(101 \mathrm{MHz}, \mathrm{CDCl}_{3}\right) \delta 170.8(\mathrm{C}=\mathrm{O}), 132.7(\mathrm{Ar} \mathrm{CH}), 132.0(\mathrm{Ar} \mathrm{CH}), 129.1\left(\mathrm{q}, \mathrm{J}_{\mathrm{CF}}=30.2 \mathrm{~Hz}\right.$, $\left.\mathrm{C}_{\mathrm{q}}-\mathrm{CF}_{3}\right), 127.5(\mathrm{Ar} \mathrm{CH}), 126.2\left(\mathrm{q}, \mathrm{J}_{\mathrm{CF}}=5.5 \mathrm{~Hz}, \mathrm{CF}_{3}\right), 124.4\left(\mathrm{q}, J_{\mathrm{CF}}=273.6 \mathrm{~Hz}, \mathrm{Ar} \mathrm{CH}\right), 61.2\left(\mathrm{OCH}_{2}\right), 38.4$ $\left(\mathrm{CH}_{2}\right), 14.2\left(\mathrm{CH}_{3}\right) .{ }^{19} \mathrm{~F}\left\{{ }^{1} \mathrm{H}\right\} \operatorname{NMR}\left(377 \mathrm{MHz}, \mathrm{CDCl}_{3}\right) \delta-60.1$.

\section{SMILES: $\mathrm{O}=\mathrm{C}(\mathrm{OCC}) \mathrm{CC} 1=\mathrm{C}(\mathrm{C}(\mathrm{F})(\mathrm{F}) \mathrm{F}) \mathrm{C}=\mathrm{CC}=\mathrm{C} 1$}

InChI = 1S/C11H11F3O2/c1-2-16-10(15)7-8-5-3-4-6-9(8)11(12,13)14/h3-6H,2,7H2,1H3

\section{Ethyl (4-phenoxyphenyl)acetate (S8)}<smiles>CCOC(=O)Cc1ccc(Oc2ccccc2)cc1</smiles>

A stirred solution of (4-phenoxyphenyl)acetic acid $(1.14 \mathrm{~g}, 5.0 \mathrm{mmol}, 1.0$ equiv) and PTSA (95 mg, $0.5 \mathrm{mmol}, 0.1$ equiv) in ethanol $(18 \mathrm{~mL})$ was heated under reflux for $18 \mathrm{~h}$. After cooling, the ethanol was evaporated in vacuo and saturated aq $\mathrm{NaHCO}_{3}(20 \mathrm{~mL})$ was added. The mixture was extracted with $\mathrm{Et}_{2} \mathrm{O}$ $(3 \times 10 \mathrm{~mL})$ and the organic phases were combined, washed with water $(2 \times 20 \mathrm{~mL})$ then brine $(20 \mathrm{~mL})$, dried over sodium sulfate and filtered. The filtrate was concentrated in vacuo afforded S8 (1.19 $\mathrm{g}, 93 \%)$ as a faint yellow liquid. $\mathrm{R}_{f}=0.19\left(5 \% \mathrm{Et}_{2} \mathrm{O}\right.$ in hexane). IR (ATR)/cm ${ }^{-1}$ 2982, $1730(\mathrm{C}=\mathrm{O}), 1588,1506,1482$, 1230, 1152, 1029, 869, 753, 693. ${ }^{1} \mathrm{H}$ NMR $\left(400 \mathrm{MHz}, \mathrm{CDCl}_{3}\right) \delta 7.36-7.30(\mathrm{~m}, 2 \mathrm{H}, \mathrm{Ar}-\mathrm{H}), 7.26-7.23(\mathrm{~m}, 2 \mathrm{H}$, $2 \times \mathrm{Ar}-\mathrm{H}), 7.12-7.08(\mathrm{~m}, 1 \mathrm{H}, \mathrm{Ar}-\mathrm{H}), 7.02-6.99(\mathrm{~m}, 2 \mathrm{H}, 2 \times \mathrm{Ar}-\mathrm{H}), 6.96(\mathrm{dt}, 2 \mathrm{H}, J=8.7,2.5 \mathrm{~Hz}, 2 \times \mathrm{Ar}-\mathrm{H})$, $4.16\left(\mathrm{q}, 2 \mathrm{H}, J=7.1 \mathrm{~Hz}, \mathrm{OCH}_{2}\right), 3.59$ (s, 2H, CH ), $1.26\left(\mathrm{t}, 3 \mathrm{H}, J=7.1 \mathrm{~Hz}, \mathrm{CH}_{3}\right) .{ }^{13} \mathrm{C} \mathrm{NMR}\left(101 \mathrm{MHz} \mathrm{CDCl}_{3}\right)$ $\delta 171.8(\mathrm{C}=\mathrm{O}), 157.3\left(\mathrm{Ar} \mathrm{C} \mathrm{q}^{-} \mathrm{O}\right), 156.5\left(\mathrm{Ar} \mathrm{C}_{\mathrm{q}}-\mathrm{O}\right), 130.7(2 \times \operatorname{Ar} \mathrm{CH}), 129.9(2 \times \mathrm{Ar} \mathrm{CH}), 129.1\left(\operatorname{Ar~} \mathrm{C}_{\mathrm{q}}\right)$, $123.4(\mathrm{Ar} \mathrm{CH}), 119.0(4 \times \mathrm{Ar} \mathrm{CH}), 61.0\left(\mathrm{OCH}_{2}\right), 40.8\left(\mathrm{CH}_{2}\right), 14.3\left(\mathrm{CH}_{3}\right)$. The observed characterisation data $\left(\mathrm{IR},{ }^{1} \mathrm{H}\right.$ and $\left.{ }^{13} \mathrm{C} \mathrm{NMR}\right)$ was consistent with that previously reported in the literature. ${ }^{9}$

SMILES: $\mathrm{O}=\mathrm{C}(\mathrm{OCC}) \mathrm{CC} 1=\mathrm{CC}=\mathrm{C}(\mathrm{OC} 2=\mathrm{CC}=\mathrm{CC}=\mathrm{C} 2) \mathrm{C}=\mathrm{C} 1$

InChI = 1S/C16H16O3/c1-2-18-16(17)12-13-8-10-15(11-9-13)19-14-6-4-3-5-7-14/h3-11H,2,12H2,1H3

\section{Ethyl 2-([1,1'-biphenyl]-4-yl)acetate (S9)}<smiles>CCOC(=O)Cc1ccc(-c2ccccc2)cc1</smiles>

A stirred solution of ([1,1'-biphenyl]-4-yl)acetic acid (1.02 g, $5.0 \mathrm{mmol}, 1.0$ equiv) and PTSA (95 mg, $0.5 \mathrm{mmol}, 0.1$ equiv) in ethanol $(12.5 \mathrm{~mL})$ was heated under reflux for $18 \mathrm{~h}$. After cooling, the ethanol was evaporated in vacuo and saturated aq $\mathrm{NaHCO}_{3}(20 \mathrm{~mL})$ was added. The mixture was extracted with $\mathrm{Et}_{2} \mathrm{O}(3$ $\times 10 \mathrm{~mL})$ and the organic phases were combined, washed with water $(2 \times 20 \mathrm{~mL})$ then brine $(20 \mathrm{~mL})$, dried over sodium sulfate and filtered. The filtrate was concentrated in vacuo afforded $\mathbf{S 9}(1.05 \mathrm{~g}, 87 \%)$ as a colourless liquid. $\mathrm{R}_{f}=0.20$ (5\% $\mathrm{Et}_{2} \mathrm{O}$ in hexane). IR (ATR)/cm ${ }^{-1}$ 2982, $1730(\mathrm{C}=\mathrm{O}), 1487,1368,1245,1148$,

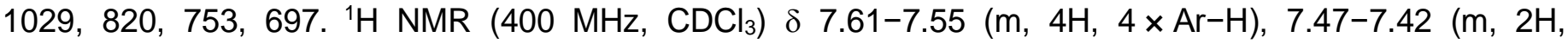
$2 \times \mathrm{Ar}-\mathrm{H}), 7.39-7.33(\mathrm{~m}, 3 \mathrm{H}, 3 \times \mathrm{Ar}-\mathrm{H}), 4.19\left(\mathrm{q}, 2 \mathrm{H}, J=7.1 \mathrm{~Hz}, \mathrm{OCH}_{2}\right), 3.67\left(\mathrm{~s}, 2 \mathrm{H}, \mathrm{CH}_{2}\right), 1.29(\mathrm{t}, 3 \mathrm{H}$, $\left.J=7.1 \mathrm{~Hz}, \mathrm{CH}_{3}\right) .{ }^{13} \mathrm{C}$ NMR $\left(101 \mathrm{MHz}, \mathrm{CDCl}_{3}\right) \delta 171.7(\mathrm{C}=\mathrm{O}), 140.9\left(\mathrm{Ar} \mathrm{C}_{\mathrm{q}}-\mathrm{Ph}\right), 140.1\left(\mathrm{Ar} \mathrm{C} \mathrm{q}_{\mathrm{q}}-\mathrm{Ph}\right), 133.3(\mathrm{Ar}$ $\left.\mathrm{C}_{\mathrm{q}}\right), 129.8(2 \times \mathrm{Ar} \mathrm{CH}), 128.9(2 \times \mathrm{Ar} \mathrm{CH}), 127.4(2 \times \mathrm{Ar} \mathrm{CH}), 127.4(\mathrm{Ar} \mathrm{CH}), 127.2(2 \times \mathrm{Ar} \mathrm{CH}), 61.1$ $\left(\mathrm{OCH}_{2}\right), 41.2\left(\mathrm{CH}_{2}\right), 14.3\left(\mathrm{CH}_{3}\right)$. The observed characterisation data $\left({ }^{1} \mathrm{H}\right.$ and $\left.{ }^{13} \mathrm{C} \mathrm{NMR}\right)$ was consistent with that previously reported in the literature. ${ }^{10,11}$ 
$\mathrm{InChI}=1 \mathrm{~S} / \mathrm{C} 16 \mathrm{H} 16 \mathrm{O} 2 / \mathrm{c} 1-2-18-16(17) 12-13-8-10-15(11-9-13) 14-6-4-3-5-7-14 / \mathrm{h} 3-11 \mathrm{H}, 2,12 \mathrm{H} 2,1 \mathrm{H} 3$

The following tosylhydrazones were prepared according to a procedure adapted from Gouverneur: ${ }^{12}$

4-Methyl-N'-(2,2,2-trifluoro-1-phenylethylidene)benzenesulfonohydrazide (S10)<smiles>FC(F)(F)/C(=N\Nc1ccccc1)c1ccccc1</smiles>

Tosylhydrazide ( $3.72 \mathrm{~g}, 20 \mathrm{mmol}, 1$ equiv) was added to trifluoroacetophenone $(2.81 \mathrm{~mL}, 20 \mathrm{mmol}, 1$ equiv) in methanol $(13 \mathrm{~mL})$ and the mixture was heated to $65^{\circ} \mathrm{C}$. After $15 \mathrm{~h}$ the mixture was cooled to $0{ }^{\circ} \mathrm{C}$ and pentane was added to aid precipitate formation. The precipitate was filtered and concentrated in vacuo affording S10 (3.14 g, 46\%) as a white crystalline solid. IR (film)/cm ${ }^{-1} 3191$ (NH), 1361, 1334, 1174, 1129, 1066, 988, 772, 682. ${ }^{1} \mathrm{H}$ NMR $\left(400 \mathrm{MHz}, \mathrm{CDCl}_{3}\right) \delta 7.99(\mathrm{~s}, 1 \mathrm{H}, \mathrm{NH}), 7.82(\mathrm{~d}, 2 \mathrm{H}, J=8.3 \mathrm{~Hz}, 2 \times \mathrm{Ar}-\mathrm{H})$, 7.58-7.50 (m, 3H, $3 \times \mathrm{Ar}-\mathrm{H}), 7.36(\mathrm{~d}, 2 \mathrm{H}, \mathrm{J}=8.2 \mathrm{~Hz}, 2 \times \mathrm{Ar}-\mathrm{H}), 7.27-7.22(\mathrm{~m}, 2 \mathrm{H}, 2 \times \mathrm{Ar}-\mathrm{H}), 2.47(\mathrm{~s}, 3 \mathrm{H}$, $\left.\mathrm{CH}_{3}\right) \cdot{ }^{13} \mathrm{C}$ NMR $\left(101 \mathrm{MHz}, \mathrm{CDCl}_{3}\right) \delta 144.9(\mathrm{Ph} \mathrm{C}), 141.6\left(\mathrm{q}, \mathrm{J}_{\mathrm{CF}}=35.9 \mathrm{~Hz}, \mathrm{C}_{\mathrm{q}}=\mathrm{N}\right), 134.5\left(\mathrm{Ts} \mathrm{C}_{\mathrm{q}}\right), 131.5(\mathrm{Ph}$ $\mathrm{CH}), 130.0(2 \times \mathrm{ArCH}), 129.8(2 \times \mathrm{Ar} \mathrm{CH}), 128.1$ (2 $\times \mathrm{Ar} \mathrm{CH}), 128.0(2 \times \mathrm{Ar} \mathrm{CH}), 125.2\left(\mathrm{C}_{\mathrm{q}}-\mathrm{CH}_{3}\right), 119.9$ (q, $\left.J_{\mathrm{CF}}=275.0 \mathrm{~Hz}, \mathrm{CF}_{3}\right), 21.7\left(\mathrm{CH}_{3}\right) .{ }^{19} \mathrm{~F}\left\{{ }^{1} \mathrm{H}\right\} \mathrm{NMR}\left(377 \mathrm{MHz}, \mathrm{CDCl}_{3}\right) \delta-63.3$. The observed characterisation data $\left({ }^{1} \mathrm{H},{ }^{13} \mathrm{C}\right.$ and ${ }^{19} \mathrm{~F}$ NMR $)$ was consistent with that previously reported in the literature. ${ }^{12}$

SMILES: $F C(C(C 1=C C=C C=C 1)=N N S(C 2=C C=C(C) C=C 2)(=O)=O)(F) F$

InChl = 1S/C15H13F3N2O2S/c1-11-7-9-13(10-8-11)23(21,22)20-19-14(15(16,17)18)12-5-3-2-4-6-12/h2$10,20 \mathrm{H}, 1 \mathrm{H} 3 / \mathrm{b} 19-14+$

N'-(1-(4-Bromophenyl)-2,2,2-trifluoroethylidene)-4-methylbenzenesulfonohydrazide (S11)<smiles>FC(F)(F)c1ccc(Br)cc1</smiles>

Tosylhydrazide (1.86 g, $10.0 \mathrm{mmol}$, 1 equiv) was added to 4-bromo-trifluoroacetophenone (2.53 g, $10.0 \mathrm{mmol}, 1$ equiv) in toluene $(5 \mathrm{~mL})$ and the mixture was heated to $100{ }^{\circ} \mathrm{C}$. After $15 \mathrm{~h}$ the mixture was cooled to $0{ }^{\circ} \mathrm{C}$ and pentane was added to aid precipitate formation. The precipitate was stirred for 30 min at $0 \stackrel{\circ}{ }$, then filtered and concentrated in vacuo affording $S 11(2.79 \mathrm{~g}, 66 \%)$ as a white crystalline solid. IR (film) $/ \mathrm{cm}^{-1}$ 3176, 1357, 1170, 1133, 1070, 999, 876, 813. ${ }^{1} \mathrm{H}$ NMR $\left(400 \mathrm{MHz}, \mathrm{CDCl}_{3}\right) \delta 8.02(\mathrm{~s}, 1 \mathrm{H}, \mathrm{NH})$, $7.80(\mathrm{~d}, 2 \mathrm{H}, J=8.3 \mathrm{~Hz}, 2 \times \mathrm{Ar}-\mathrm{H}), 7.67(\mathrm{~d}, 2 \mathrm{H}, J=8.5 \mathrm{~Hz}, 2 \times \mathrm{Ar}-\mathrm{H}), 7.37(\mathrm{~d}, 2 \mathrm{H}, J=8.3 \mathrm{~Hz}, 2 \times \mathrm{Ar}-\mathrm{H})$, $7.14(\mathrm{~d}, 2 \mathrm{H}, J=8.5 \mathrm{~Hz}, 2 \times \mathrm{Ar}-\mathrm{H}), 2.47\left(\mathrm{~s}, 3 \mathrm{H}, \mathrm{CH}_{3}\right) \cdot{ }^{13} \mathrm{C} \mathrm{NMR}\left(101 \mathrm{MHz}, \mathrm{CDCl}_{3}\right) \delta 145.1\left(\operatorname{Ar} \mathrm{C}_{\mathrm{q}}\right), 140.2(\mathrm{q}$, $\left.J_{\mathrm{CF}}=35.7 \mathrm{~Hz}, \mathrm{C}_{\mathrm{q}}=\mathrm{N}\right), 134.3\left(\mathrm{Ts} \mathrm{C}_{\mathrm{q}}\right), 133.3(2 \times \mathrm{Ar} \mathrm{CH}), 129.9(2 \times \mathrm{Ar} \mathrm{CH}), 129.8(2 \times \mathrm{Ar} \mathrm{CH}), 128.0(2 \times \mathrm{Ar}$ $\mathrm{CH}), 126.4$ and 123.9 ( $\mathrm{Ar} \mathrm{C} \mathrm{q}_{-}-\mathrm{Br}$ and $\left.\mathrm{Ar} \mathbf{C}_{\mathrm{q}}-\mathrm{CH}_{3}\right), 119.7\left(\mathrm{q}, \mathrm{J}_{\mathrm{CF}}=274.8 \mathrm{~Hz}, \mathrm{CF}_{3}\right), 21.7\left(\mathrm{CH}_{3}\right) .{ }^{19} \mathrm{~F}\left\{{ }^{1} \mathrm{H}\right\} \mathrm{NMR}$ $\left(377 \mathrm{MHz} \mathrm{CDCl}_{3}\right) \delta-68.2$. The observed characterisation data $\left({ }^{1} \mathrm{H},{ }^{13} \mathrm{C}\right.$ and $\left.{ }^{19} \mathrm{~F} \mathrm{NMR}\right)$ was consistent with that previously reported in the literature. ${ }^{12}$

SMILES: $\mathrm{BrC1}=\mathrm{CC}=\mathrm{C}(\mathrm{C}(\mathrm{C}(\mathrm{F})(\mathrm{F}) \mathrm{F})=\mathrm{NNS}(\mathrm{C} 2=\mathrm{CC}=\mathrm{C}(\mathrm{C}) \mathrm{C}=\mathrm{C} 2)(=\mathrm{O})=\mathrm{O}) \mathrm{C}=\mathrm{C} 1$

InChI = 1S/C15H12BrF3N2O2S/c1-10-2-8-13(9-3-10)24(22,23)21-20-14(15(17,18)19)11-4-6-12(16)7-511/h2-9,21H,1H3/b20-14+ 


\section{Diazo compounds:}

The following diazo compounds were prepared as previously reported. ${ }^{13-16}$

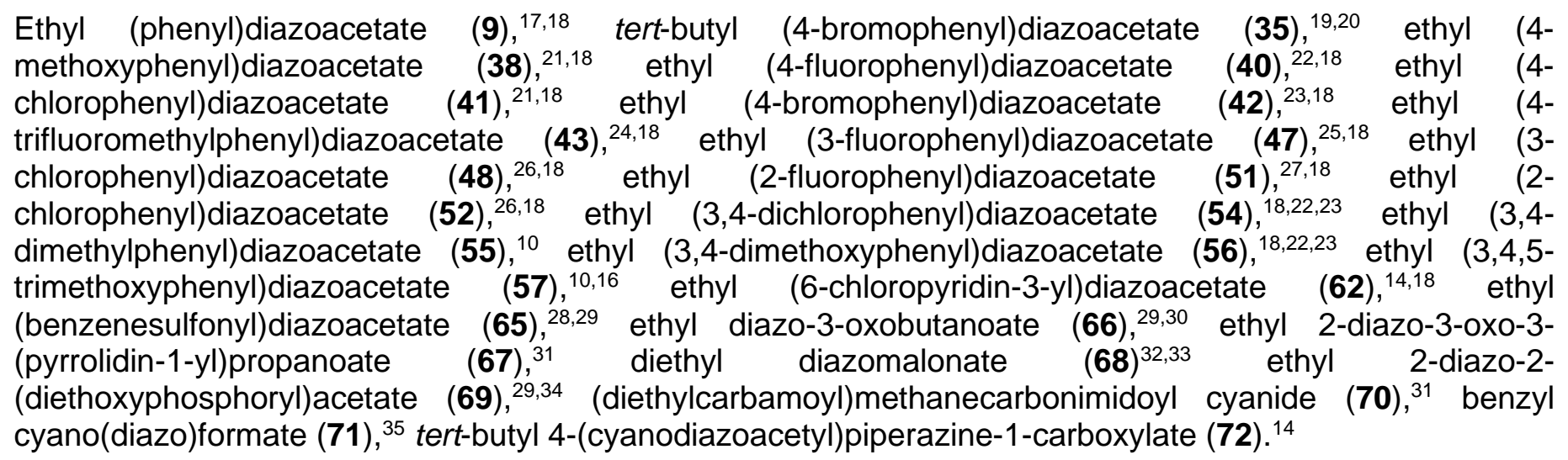

The following diazo compounds were synthesised using a procedure adapted from Davies and coworkers: ${ }^{18}$

\section{Methyl (phenyl)diazoacetate (10)}<smiles>COC(=O)C(=N)c1ccccc1</smiles>

DBU $(0.9 \mathrm{~mL}, 6.0 \mathrm{mmol}, 1.2$ equiv) was added dropwise to a stirred solution of $p$-ABSA (1.44 g, $6.0 \mathrm{mmol}$, 1.2 equiv) and methyl (phenyl)acetate $\left(750 \mathrm{mg}, 5.0 \mathrm{mmol}, 1.0\right.$ equiv) in $\mathrm{MeCN}(10 \mathrm{~mL})$ at $0{ }^{\circ} \mathrm{C}$. The mixture was then warmed to $25^{\circ} \mathrm{C}$ and stirred for $24 \mathrm{~h}$. Saturated aq $\mathrm{NH}_{4} \mathrm{Cl}(30 \mathrm{~mL})$ was added. The aqueous mixture was extracted with $\mathrm{Et}_{2} \mathrm{O}(3 \times 30 \mathrm{~mL})$. The organic extracts were dried over sodium sulfate, filtered and concentrated in vacuo. The residue was then purified by column chromatography using $3 \% \mathrm{Et}_{2} \mathrm{O}$ in pentane, affording $10(560 \mathrm{mg}, 64 \%)$ as a bright orange liquid. $\mathrm{R}_{f}=0.49\left(3 \% \mathrm{Et}_{2} \mathrm{O}\right.$ in pentane). IR $($ ATR $) / \mathrm{cm}^{-1}$ 2953, $2080(\mathrm{~N}=\mathrm{N}), 1698(\mathrm{C}=\mathrm{O}), 1598,1498,1434,1351,1246,1191,1152,1051,1024,753$, 690. ${ }^{1} \mathrm{H}$ NMR $\left(400 \mathrm{MHz}, \mathrm{CDCl}_{3}\right) \delta 7.48(\mathrm{dd}, 2 \mathrm{H}, J=8.6,1.2 \mathrm{~Hz}, 2 \times \mathrm{Ar}-\mathrm{H}), 7.39(\mathrm{t}, 2 \mathrm{H}, J=8.0 \mathrm{~Hz}$, $2 \times \mathrm{Ar}-\mathrm{H}), 7.19(\mathrm{tt}, 1 \mathrm{H}, J=7.4,1.2 \mathrm{~Hz}, \mathrm{Ar}-\mathrm{H}), 3.87\left(\mathrm{~s}, 3 \mathrm{H}, \mathrm{OCH}_{3}\right) .{ }^{13} \mathrm{C} \mathrm{NMR}\left(101 \mathrm{MHz}, \mathrm{CDCl}_{3}\right) \delta 165.8$ $(\mathrm{C}=\mathrm{O}), 129.1(2 \times \mathrm{ArCH}), 126.0(\mathrm{Ar} \mathrm{CH}), 125.6\left(\mathrm{Ar} \mathrm{C} \mathrm{C}_{\mathrm{q}}\right), 124.1(2 \times \mathrm{Ar} \mathrm{CH}), 52.1\left(\mathrm{OCH}_{3}\right)$. The observed characterisation data (IR, ${ }^{1} \mathrm{H}$ and ${ }^{13} \mathrm{C}$ NMR) was consistent with that previously reported in the literature. ${ }^{36,37}$

SMILES: $\mathrm{O}=\mathrm{C}(\mathrm{OC}) \mathrm{C}(\mathrm{C} 1=\mathrm{CC}=\mathrm{CC}=\mathrm{C} 1)=[\mathrm{N}+]=[\mathrm{N}-]$

InChl = 1S/C9H8N2O2/c1-13-9(12)8(11-10)7-5-3-2-4-6-7/h2-6H,1H3 


\section{tert-Butyl (phenyl)diazoacetate (34)}<smiles>CCCCOC(=O)C(=N)c1ccccc1</smiles>

DBU $(0.15 \mathrm{~mL}, 0.94 \mathrm{mmol}, 1.2$ equiv) was added dropwise to a stirred solution of $p$-ABSA (226 mg, 0.94 mmol, 1.2 equiv) and tert-butyl (phenyl)acetate $\mathbf{S 1}(150 \mathrm{mg}, 0.78 \mathrm{mmol}, 1.0$ equiv) in MeCN (2 mL) at $0{ }^{\circ} \mathrm{C}$. The mixture was then warmed to $25^{\circ} \mathrm{C}$ and stirred for $20 \mathrm{~h}$. Saturated aq $\mathrm{NH}_{4} \mathrm{Cl}(12 \mathrm{~mL})$ was added. The aqueous mixture was extracted with $\mathrm{Et}_{2} \mathrm{O}(3 \times 10 \mathrm{~mL})$. The organic extracts were dried over sodium sulfate, filtered and concentrated in vacuo. The residue was then purified by column chromatography using $1 \% \mathrm{Et}_{2} \mathrm{O}$ in pentane, affording $34(75 \mathrm{mg}, 44 \%)$ as a bright orange liquid. $\mathrm{R}_{f}=0.37$ (1\% $\mathrm{Et}_{2} \mathrm{O}$ in pentane). IR (ATR) $/ \mathrm{cm}^{-1}$ 2978, 2931, $2077(\mathrm{~N}=\mathrm{N}), 1696(\mathrm{C}=\mathrm{O})$, 1498, 1450, 1368, 1349, 1287, 1245, 1140, 1007, 754, 690. ${ }^{1} \mathrm{H}$ NMR $\left(400 \mathrm{MHz}, \mathrm{CDCl}_{3}\right) \delta 7.47(\mathrm{~d}, 2 \mathrm{H}, J=7.5 \mathrm{~Hz}, 2 \times \mathrm{Ar}-\mathrm{H}), 7.37(\mathrm{t}, 2 \mathrm{H}, J=7.5 \mathrm{~Hz}, 2 \times \mathrm{Ar}-\mathrm{H}), 7.16$ (t, $1 \mathrm{H}, J=7.5 \mathrm{~Hz}, \mathrm{Ar}-\mathrm{H}), 1.55\left(\mathrm{~s}, 9 \mathrm{H}, 3 \times \mathrm{CH}_{3}\right) \cdot{ }^{13} \mathrm{C} \mathrm{NMR}\left(101 \mathrm{MHz}, \mathrm{CDCl}_{3}\right) \delta 164.7(\mathrm{C}=\mathrm{O}), 130.0(2 \times \mathrm{Ar}$ $\mathrm{CH}), 126.3\left(\mathrm{Ar} \mathrm{C}_{\mathrm{q}}\right), 125.7(\mathrm{Ar} \mathrm{CH}), 124.1(2 \times \mathrm{Ar} \mathrm{CH}), 82.2\left(t \mathrm{Bu} \mathrm{C} \mathrm{C}_{\mathrm{q}}\right), 28.5\left(3 \times \mathrm{CH}_{3}\right)$. The observed characterisation data (IR, $1 \mathrm{H}$ and $13 \mathrm{C}$ NMR) was consistent with that previously reported in the literature. ${ }^{38}$

SMILES: $\mathrm{O}=\mathrm{C}(\mathrm{OC}(\mathrm{C})(\mathrm{C}) \mathrm{C}) \mathrm{C}(\mathrm{C} 1=\mathrm{CC}=\mathrm{CC}=\mathrm{C} 1)=[\mathrm{N}+]=[\mathrm{N}-]$

InChI = 1S/C12H14N2O2/c1-12(2,3)16-11(15)10(14-13)9-7-5-4-6-8-9/h4-8H,1-3H3

$(1 R, 2 S, 5 R)-2-$ Isopropyl-5-methylcyclohexyl (phenyl)diazoacetate (36)<smiles>CC(C)[C@H]1CC[C@@H](C)C[C@H]1OC(=O)C(=N)c1ccccc1</smiles>

DBU $(0.54 \mathrm{~mL}, 3.6 \mathrm{mmol}, 1.2$ equiv) was added dropwise over 2 min to a stirred solution of $p$-ABSA (865 mg, $3.6 \mathrm{mmol}, 1.2$ equiv) and $\mathbf{S 2}\left(823 \mathrm{mg}, 3.0 \mathrm{mmol}, 1.0\right.$ equiv) in MeCN $(5 \mathrm{~mL})$ at $0{ }^{\circ} \mathrm{C}$. The mixture was then warmed to $25^{\circ} \mathrm{C}$ and stirred for $24 \mathrm{~h}$. Saturated aq $\mathrm{NH}_{4} \mathrm{Cl}(50 \mathrm{~mL})$ was added. The aqueous mixture was extracted with $\mathrm{Et}_{2} \mathrm{O}(3 \times 50 \mathrm{~mL}) .50 \% \mathrm{Et}_{2} \mathrm{O}$ in pentane $(50 \mathrm{~mL})$ was added and the mixture was filtered, and solids washed with $50 \% \mathrm{Et}_{2} \mathrm{O}$ in pentane $(50 \mathrm{~mL})$. The combined organics were dried over sodium sulfate and concentrated in vacuo. Purification by flash chromatography using $2 \% \mathrm{Et}_{2} \mathrm{O}$ in pentane afforded 36 (682 mg, 76\%) as an orange oil. $\mathrm{R}_{f}=0.35\left(2 \% \mathrm{Et}_{2} \mathrm{O}\right.$ in pentane). IR (film)/cm ${ }^{-1} 2955,2925$, 2867, 2079 ( $\mathrm{C}=\mathrm{N}=\mathrm{N}$ in-phase), $1697(\mathrm{C}=\mathrm{O}), 1498,1450,1368$ ( $\mathrm{C}=\mathrm{N}=\mathrm{N}$ out-of-phase), 1353, 1253, 1239, 1163, 1148, 1040, 1013, 752, 690, 670. ${ }^{1} \mathrm{H}$ NMR $\left(400 \mathrm{MHz}, \mathrm{CDCl}_{3}\right) \delta 7.53-7.48(\mathrm{~m}, 2 \mathrm{H}, 2 \times \mathrm{Ar}-\mathrm{H})$, 7.42-7.36 (m, 2H, $2 \times \mathrm{Ar}-\mathrm{H}), 7.21-7.16(\mathrm{~m}, 1 \mathrm{H}, \mathrm{Ar}-\mathrm{H}), 4.89(\mathrm{td}, 1 \mathrm{H}, \mathrm{J}=10.9,4.4 \mathrm{~Hz}, \mathrm{OCH}), 2.16-2.10(\mathrm{~m}$, $\left.2 \mathrm{H},\left(\mathrm{CH}\left(\mathrm{CH}_{3}\right)_{2}\right) \mathrm{CH}\right), 1.93\left(\mathrm{pd}, 1 \mathrm{H}, J=6.9,2.7 \mathrm{~Hz} \mathrm{CH} \mathrm{CH}_{3}, 1.75-1.68\left(\mathrm{~m}, 2 \mathrm{H}, \mathrm{OCHCH}_{2}\right), 1.60-1.41(\mathrm{~m}, 2 \mathrm{H}\right.$, $\left.\mathrm{PrCHCH}_{2}\right), 1.18-1.03\left(\mathrm{~m}, 2 \mathrm{H}, \mathrm{CH}_{3} \mathrm{CHCH}_{2}\right), 0.93\left(\mathrm{dd}, 6 \mathrm{H}, J=6.8,5.3 \mathrm{~Hz},\left(\mathrm{CH}_{3}\right)_{2} \mathrm{CH}\right), 0.82(\mathrm{~d}, 3 \mathrm{H}, J=6.9 \mathrm{~Hz}$, $\left.\mathrm{CHCH}_{3}\right) .{ }^{13} \mathrm{C}$ NMR $\left(101 \mathrm{MHz}, \mathrm{CDCl}_{3}\right) \delta 164.7(\mathrm{C}=\mathrm{O}), 128.9(2 \times \mathrm{Ar} \mathrm{CH}), 125.8\left(\mathrm{Ar} \mathrm{C}_{\mathrm{q}}\right), 125.6(\mathrm{Ar} \mathrm{CH}), 123.9$ $(2 \times \operatorname{Ar~CH}), 75.0(\mathrm{OCH}), 63.3\left(\mathrm{C}_{q}=\mathrm{N}_{2}\right), 47.1(\mathrm{PrCH}), 41.3\left(\mathrm{OCHCH}_{2}\right), 34.2\left(\mathrm{MeCHCH}_{2}\right), 31.4(\mathrm{MeCH}), 26.5$ $\left(\mathrm{CHCH}(\mathrm{Me})_{2}\right), 23.7\left(\mathrm{CH}_{2} \mathrm{CH}(\mathrm{iPr})\right), 22.0\left(\mathrm{CH}\left(\mathrm{CH}_{3}\right) \mathrm{CH}_{3}\right), 20.7\left(\mathrm{CH}\left(\mathrm{CH}_{3}\right) \mathbf{C H}_{3}\right), 16.6\left(\mathrm{CH}\left(\mathrm{CH}_{3}\right)\right)$. The observed characterisation data (IR, ${ }^{1} \mathrm{H}$ and ${ }^{13} \mathrm{C}$ NMR) was consistent with that previously reported in the literature. ${ }^{39}$

SMILES: O=C(O[C@H]1[C@H](C(C)C)CC[C@@H](C)C1)C(C2=CC=CC=C2)=[N+]=[N-]

InChI = 1S/C18H24N2O2/c1-12(2)15-10-9-13(3)11-16(15)22-18(21)17(20-19)14-7-5-4-6-8-14/h4-8,12$13,15-16 \mathrm{H}, 9-11 \mathrm{H} 2,1-3 \mathrm{H} 3 / \mathrm{t} 13-, 15+, 16-/ \mathrm{m} 1 / \mathrm{s} 1$ 


\section{2,2,2-Trichloroethyl (phenyl)diazoacetate (37)}<smiles>N#CC(=O)OCC(Cl)(Cl)Cl</smiles>

DBU (0.85 mL, $5.50 \mathrm{mmol}, 2.2$ equiv) was added dropwise to a stirred solution of o-NBSA (856 mg, $3.75 \mathrm{mmol}, 1.5$ equiv) and 2,2,2-trichloroethyl (phenyl)acetate S3 (669 mg, $2.50 \mathrm{mmol}, 1.0$ equiv) in MeCN $(16 \mathrm{~mL})$ at $0{ }^{\circ} \mathrm{C}$ and stirred for $3 \mathrm{~h}$. Saturated aq $\mathrm{NH}_{4} \mathrm{Cl}(15 \mathrm{~mL})$ was added. The aqueous mixture was extracted with $\mathrm{Et}_{2} \mathrm{O}(3 \times 20 \mathrm{~mL})$. The organic extracts were concentrated in vacuo, and to the resulting residue hexane $(50 \mathrm{~mL})$ was added. The solution was filtered and washed with hexane $(50 \mathrm{~mL})$, dried over sodium sulfate, filtered and concentrated in vacuo. Purification by column chromatography using $2 \% \mathrm{Et}_{2} \mathrm{O}$ in pentane, afforded 37 (492 mg, 67\%) as a bright yellow solid. $\mathrm{R}_{f}=0.22\left(2 \% \mathrm{Et}_{2} \mathrm{O}\right.$ in pentane). IR (ATR)/cm ${ }^{-1}$ 2993, $2095(\mathrm{~N}=\mathrm{N}), 1700(\mathrm{C}=\mathrm{O})$, 1498, 1450, 1379, 1334, 1234, 1148, 1051, 947, 753, 712. ${ }^{1} \mathrm{H}$ NMR $\left(400 \mathrm{MHz}, \mathrm{CDCl}_{3}\right) \delta 7.52-7.49(\mathrm{~m}, 2 \mathrm{H}, 2 \times \mathrm{Ar}-\mathrm{H}), 7.44-7.39(\mathrm{~m}, 2 \mathrm{H}, 2 \times \mathrm{Ar}-\mathrm{H}), 7.25-7.20(\mathrm{~m}, 1 \mathrm{H}, \mathrm{Ar}-\mathrm{H})$, $4.92\left(\mathrm{~s}, 2 \mathrm{H}, \mathrm{OCH}_{2}\right) .{ }^{13} \mathrm{C} \mathrm{NMR}\left(101 \mathrm{MHz}, \mathrm{CDCl}_{3}\right) \delta 163.5(\mathrm{C}=\mathrm{O}), 129.2(2 \times \mathrm{Ar} \mathrm{CH}), 126.5(2 \times \mathrm{Ar} \mathrm{CH}), 124.8$ $\left(\operatorname{Ar~} \mathrm{C}_{\mathrm{q}}\right), 124.2(\mathrm{Ar} \mathrm{CH}), 95.2\left(\mathrm{CCl}_{3}\right), 74.0\left(\mathrm{OCH}_{2}\right)$. The observed characterisation data $\left(\mathrm{IR},{ }^{1} \mathrm{H}\right.$ and ${ }^{13} \mathrm{C}$ NMR) was consistent with that previously reported in the literature. ${ }^{40}$

SMILES: $\mathrm{O}=\mathrm{C}(\mathrm{OCC}(\mathrm{Cl})(\mathrm{Cl}) \mathrm{Cl}) \mathrm{C}(\mathrm{C} 1=\mathrm{CC}=\mathrm{CC}=\mathrm{C} 1)=[\mathrm{N}+]=[\mathrm{N}-]$

InChI = 1S/C10H7Cl3N2O2/c11-10(12,13)6-17-9(16)8(15-14)7-4-2-1-3-5-7/h1-5H,6H2

\section{Ethyl (4-methylphenyl)diazoacetate (39)}<smiles>CCOC(=O)C(=N)c1ccc(C)cc1</smiles>

DBU (0.18 mL, $1.2 \mathrm{mmol}, 1.2$ equiv) was added dropwise to a stirred solution of $p$-ABSA (288 mg, $1.2 \mathrm{mmol}$, 1.2 equiv) and ethyl (4-methylphenyl)acetate $\left(204 \mathrm{mg}, 1.0 \mathrm{mmol}, 1.0\right.$ equiv) in $\mathrm{MeCN}(2 \mathrm{~mL})$ at $0{ }^{\circ} \mathrm{C}$. The mixture was then warmed to $25^{\circ} \mathrm{C}$ and stirred for $24 \mathrm{~h}$. Saturated aq $\mathrm{NH}_{4} \mathrm{Cl}(10 \mathrm{~mL})$ was added. The aqueous mixture was extracted with $\mathrm{Et}_{2} \mathrm{O}(3 \times 10 \mathrm{~mL})$. The organic extracts were concentrated in vacuo, and to the resulting residue hexane $(30 \mathrm{~mL})$ was added. The solution was filtered and washed with hexane $(30 \mathrm{~mL})$, dried over sodium sulfate, filtered and concentrated in vacuo. Purification by column chromatography using $3 \% \mathrm{Et}_{2} \mathrm{O}$ in pentane, afforded $39(98 \mathrm{mg}, 48 \%)$ as an orange solid. $\mathrm{R}_{f}=0.59(3 \%$ $\mathrm{Et}_{2} \mathrm{O}$ in pentane). IR (ATR)/cm $\mathrm{cm}^{-1}$ 2981, $2078(\mathrm{~N}=\mathrm{N}), 1698(\mathrm{C}=\mathrm{O}), 1514,1445,1369,1338,1243,1150,1044$, 809, 739. ${ }^{1} \mathrm{H}$ NMR (400 MHz, $\left.\mathrm{CDCl}_{3}\right) \delta 7.36(\mathrm{~d}, 2 \mathrm{H}, J=8.3 \mathrm{~Hz}, 2 \times \mathrm{Ar}-\mathrm{H}), 7.20(\mathrm{~d}, 2 \mathrm{H}, J=8.0,2 \times \mathrm{Ar}-\mathrm{H})$, $4.33\left(\mathrm{q}, 2 \mathrm{H}, J=7.1 \mathrm{~Hz}, \mathrm{OCH}_{2}\right), 2.34(\mathrm{~s}, 3 \mathrm{H}, \mathrm{Ar}-\mathrm{Me}), 1.34\left(\mathrm{t}, 3 \mathrm{H}, J=7.1 \mathrm{~Hz}, \mathrm{CH}_{3}\right) .{ }^{13} \mathrm{C} \mathrm{NMR}(101 \mathrm{MHz}$, $\left.\mathrm{CDCl}_{3}\right) \delta 165.6(\mathrm{C}=\mathrm{O}), 135.8\left(\mathrm{Ar} \mathrm{C}_{\mathrm{q}}-\mathrm{Me}\right), 129.8(2 \times \mathrm{Ar} \mathrm{CH}), 124.3(2 \times \mathrm{Ar} \mathrm{CH}), 122.5\left(\operatorname{Ar~} \mathrm{C}_{\mathrm{q}}\right), 61.1\left(\mathrm{OCH}_{2}\right)$, $21.1\left(\mathrm{Ar}-\mathrm{CH}_{3}\right), 14.7\left(\mathrm{CH}_{3}\right)$. The observed characterisation data $\left({ }^{1} \mathrm{H}\right.$ and $\left.{ }^{13} \mathrm{C} \mathrm{NMR}\right)$ was consistent with that previously reported in the literature. ${ }^{21,41}$

SMILES: $\mathrm{O}=\mathrm{C}(\mathrm{OCC}) \mathrm{C}(\mathrm{C} 1=\mathrm{CC}=\mathrm{C}(\mathrm{C}) \mathrm{C}=\mathrm{C} 1)=[\mathrm{N}+]=[\mathrm{N}-]$

InChI = 1S/C11H12N2O2/c1-3-15-11(14)10(13-12)9-6-4-8(2)5-7-9/h4-7H,3H2,1-2H3 


\section{Ethyl (4-nitrophenyl)diazoacetate (44)}<smiles>CCOC(=O)C(=N)c1ccc([N+](=O)[O-])cc1</smiles>

DBU ( $0.25 \mathrm{~mL}, 1.50 \mathrm{mmol} 1.2$ equiv) was added dropwise to a stirred solution of ethyl (4-nitrophenyl)acetate (262 mg $1.25 \mathrm{mmol}, 1.0$ equiv) and $p$-ABSA (361 mg, $1.50 \mathrm{mmol}, 1.2$ equiv) in $\mathrm{MeCN}\left(3 \mathrm{~mL}\right.$ ) at $0{ }^{\circ} \mathrm{C}$. The mixture was then warmed to $25^{\circ} \mathrm{C}$ and stirred for $24 \mathrm{~h}$. The reaction was quenched with saturated aq $\mathrm{NH}_{4} \mathrm{Cl}$ $(15 \mathrm{~mL})$ and the mixture extracted with $\mathrm{Et}_{2} \mathrm{O}(3 \times 15 \mathrm{~mL})$. The organic extracts were washed with brine $(15 \mathrm{~mL})$, dried over sodium sulfate and filtered. The filtrate was concentrated in vacuo and purified by column chromatography using $5 \% \mathrm{Et}_{2} \mathrm{O}$ in pentane, affording $44(175 \mathrm{mg}, 60 \%)$ as a bright yellow solid. $\mathrm{R}_{f}=0.30\left(5 \% \mathrm{Et}_{2} \mathrm{O}\right.$ in pentane). $\mathrm{mp}\left(\mathrm{DSC}\right.$ peak) $=85^{\circ} \mathrm{C} . \mathrm{IR}(\mathrm{ATR}) / \mathrm{cm}^{-1} 2989,2091(\mathrm{~N}=\mathrm{N}), 1689(\mathrm{C}=\mathrm{O})$, $1588,1495,1320,1234,1152,1036,842,749,686 .{ }^{1} \mathrm{H} \mathrm{NMR}\left(400 \mathrm{MHz}, \mathrm{CDCl}_{3}\right) \delta 8.23(\mathrm{t}, 2 \mathrm{H}, J=9.2 \mathrm{~Hz}$, $2 \times \mathrm{Ar}-\mathrm{H}), 7.67(\mathrm{t}, 2 \mathrm{H}, J=9.2 \mathrm{~Hz}, 2 \times \mathrm{Ar}-\mathrm{H}), 4.37\left(\mathrm{q}, 2 \mathrm{H}, J=7.1 \mathrm{~Hz}, \mathrm{OCH}_{2}\right), 1.37\left(\mathrm{t}, 3 \mathrm{H}, J=7.1 \mathrm{~Hz}, \mathrm{CH}_{3}\right)$. ${ }^{13} \mathrm{C} \mathrm{NMR}\left(101 \mathrm{MHz}, \mathrm{CDCl}_{3}\right) \delta 163.9(\mathrm{C}=\mathrm{O}), 145.2\left(\mathrm{Ar} \mathrm{C}_{\mathrm{q}}-\mathrm{NO}_{2}\right), 134.2\left(\mathrm{Ar} \mathrm{C}_{\mathrm{q}}\right), 124.5(2 \times \mathrm{Ar} \mathrm{CH}), 123.3$ $(2 \times \mathrm{Ar} \mathrm{CH}), 61.8\left(\mathrm{OCH}_{2}\right), 14.6\left(\mathrm{CH}_{3}\right)$. The observed characterisation data $\left({ }^{1} \mathrm{H}\right.$ and $\left.{ }^{13} \mathrm{C} \mathrm{NMR}\right)$ are consistent with that previously reported in the literature. ${ }^{17,25}$

SMILES: $\mathrm{O}=\mathrm{C}(\mathrm{OCC}) \mathrm{C}(\mathrm{C} 1=\mathrm{CC}=\mathrm{C}([\mathrm{N}+]([\mathrm{O}-])=\mathrm{O}) \mathrm{C}=\mathrm{C} 1)=[\mathrm{N}+]=[\mathrm{N}-]$

$\mathrm{InChI}=1 \mathrm{~S} / \mathrm{C} 10 \mathrm{H} 9 \mathrm{~N} 3 \mathrm{O} 4 / \mathrm{c} 1-2-17-10(14) 9(12-11) 7-3-5-8(6-4-7) 13(15) 16 / \mathrm{h} 3-6 \mathrm{H}, 2 \mathrm{H} 2,1 \mathrm{H} 3$

\section{Ethyl (3-methoxyphenyl)diazoacetate (45)}<smiles>CCOC(=O)C(=N)c1cccc(OC)c1</smiles>

DBU $(0.9 \mathrm{~mL}, 6.0 \mathrm{mmol}, 1.2$ equiv) was added dropwise to a stirred solution of $p$-ABSA (1.44 g, $6.0 \mathrm{mmol}$, 1.2 equiv) and ethyl (3-methoxyphenyl)acetate $\left(0.97 \mathrm{~g}, 5.0 \mathrm{mmol}, 1.0\right.$ equiv) in $\mathrm{MeCN}(10 \mathrm{~mL})$ at $0{ }^{\circ} \mathrm{C}$. The mixture was then warmed to $25{ }^{\circ} \mathrm{C}$ and stirred for $24 \mathrm{~h}$. Saturated aq $\mathrm{NH}_{4} \mathrm{Cl}(30 \mathrm{~mL})$ was added. The mixture was extracted with $\mathrm{Et}_{2} \mathrm{O}(3 \times 30 \mathrm{~mL})$. The organic extracts were dried over sodium sulfate, filtered and concentrated in vacuo. The residue was then purified by column chromatography using $5 \% \mathrm{Et}_{2} \mathrm{O}$ in pentane, affording $45(0.75 \mathrm{~g}, 68 \%)$ as a bright orange solid. $\mathrm{R}_{f}=0.47\left(5 \% \mathrm{Et}_{2} \mathrm{O}\right.$ in pentane). $\mathrm{mp}$ (DSC peak $)=51^{\circ} \mathrm{C} . \mathrm{IR}($ ATR $) / \mathrm{cm}^{-1}$ 2979, $2084(\mathrm{~N}=\mathrm{N}), 1688(\mathrm{C}=\mathrm{O}), 1605,1577,1455,1374,1246,1226,1145$, 1034, 874, 768, 681. ${ }^{1} \mathrm{H}$ NMR $\left(400 \mathrm{MHz}, \mathrm{CDCl}_{3}\right) \delta 7.29$ (app t, $\left.1 \mathrm{H}, J=8.1 \mathrm{~Hz}, \mathrm{Ar}-\mathrm{H}\right), 7.17$ (app t, $1 \mathrm{H}$, $J=2.2 \mathrm{~Hz}, \mathrm{Ar}-\mathrm{H}$ ), 6.99 (ddd, $1 \mathrm{H}, J=7.9,1.8,0.8 \mathrm{~Hz}, \mathrm{Ar}-\mathrm{H}$ ), 6.73 (ddd, $1 \mathrm{H}, J=8.1,2.5,0.8 \mathrm{~Hz}, \mathrm{Ar}-\mathrm{H}$ ), 4.33 (q, 2H, J = 7.1 Hz, OCH 2 ), $3.82(\mathrm{~s}, 3 \mathrm{H}, \mathrm{OMe}), 1.34$ (t, 3H, $\left.J=7.1 \mathrm{~Hz}, \mathrm{CH}_{3}\right) \cdot{ }^{13} \mathrm{C} \mathrm{NMR}\left(101 \mathrm{MHz}, \mathrm{CDCl}_{3}\right)$ $\delta 165.3\left(\mathrm{Ar} \mathrm{C}_{\mathrm{q}}-\mathrm{OMe}\right), 160.2(\mathrm{C}=\mathrm{O}), 130.0(\mathrm{Ar} \mathrm{CH}), 127.3\left(\mathrm{Ar} \mathrm{C}_{\mathrm{q}}\right), 116.1(\mathrm{Ar} \mathrm{CH}), 111.7(\mathrm{Ar} \mathrm{CH}), 109.8(\mathrm{Ar}$ $\mathrm{CH}), 61.1\left(\mathrm{OCH}_{2}\right), 55.41(\mathrm{OMe}), 14.6\left(\mathrm{CH}_{3}\right)$. The observed characterisation data $\left(\mathrm{IR},{ }^{1} \mathrm{H}\right.$ and $\left.{ }^{13} \mathrm{C} \mathrm{NMR}\right)$ was consistent with that previously reported in the literature. ${ }^{42}$

SMILES: $\mathrm{O}=\mathrm{C}(\mathrm{OCC}) \mathrm{C}(\mathrm{C} 1=\mathrm{CC}(\mathrm{OC})=\mathrm{CC}=\mathrm{C} 1)=[\mathrm{N}+]=[\mathrm{N}-]$

InChI = 1S/C11H12N2O3/c1-3-16-11(14)10(13-12)8-5-4-6-9(7-8)15-2/h4-7H,3H2,1-2H3 


\section{Ethyl (3-methylphenyl)diazoacetate (46)}<smiles>CCOC(=O)C(=N)c1cccc(C)c1</smiles>

DBU $(0.9 \mathrm{~mL}, 6.0 \mathrm{mmol}, 1.2$ equiv) was added dropwise to a stirred solution of $p$-ABSA (1.44 g, $6.0 \mathrm{mmol}$, 1.2 equiv) and ethyl (3-methylphenyl)acetate $\mathbf{S} 4\left(0.89 \mathrm{~g}, 5.0 \mathrm{mmol}, 1.0\right.$ equiv) in $\mathrm{MeCN}(10 \mathrm{~mL})$ at $0{ }^{\circ} \mathrm{C}$. The mixture was then warmed to $25{ }^{\circ} \mathrm{C}$ and stirred for $24 \mathrm{~h}$. Saturated aq $\mathrm{NH}_{4} \mathrm{Cl}(30 \mathrm{~mL})$ was added. The aqueous mixture was extracted with $\mathrm{Et}_{2} \mathrm{O}(3 \times 30 \mathrm{~mL})$. The organic extracts were dried over sodium sulfate, filtered and concentrated in vacuo. The residue was then purified by column chromatography using $5 \% \mathrm{Et}_{2} \mathrm{O}$ in pentane affording $46(0.63 \mathrm{~g}, 62 \%)$ as a bright orange liquid. $\mathrm{R}_{f}=0.68\left(5 \% \mathrm{Et}_{2} \mathrm{O}\right.$ in pentane $)$. IR (ATR)/cm ${ }^{-1}$ 2981, $2079(\mathrm{~N}=\mathrm{N}), 1700(\mathrm{C}=\mathrm{O}), 1604,1492,1445,1369,1245,1144,1094,1054,777,690 .{ }^{1} \mathrm{H}$ NMR (400 MHz, CDCl $) \delta$ 7.31-7.26 (m, 3H, $3 \times \mathrm{Ar}-\mathrm{H}), 6.99(\mathrm{~m}, 1 \mathrm{H}, \mathrm{Ar}-\mathrm{H}), 4.33\left(\mathrm{q}, 2 \mathrm{H}, J=7.1 \mathrm{~Hz}, \mathrm{OCH}_{2}\right)$, $2.36(\mathrm{~s}, 3 \mathrm{H}, \mathrm{Ar}-\mathrm{Me}), 1.34\left(\mathrm{t}, 3 \mathrm{H}, J=7.1 \mathrm{~Hz}, \mathrm{CH}_{3}\right) .{ }^{13} \mathrm{C} \mathrm{NMR}\left(101 \mathrm{MHz}, \mathrm{CDCl}_{3}\right) \delta 165.4(\mathrm{C}=\mathrm{O}), 138.7(\mathrm{Ar}$ $\left.\mathrm{C}_{\mathrm{q}}-\mathrm{Me}\right), 128.9(\mathrm{ArCH}), 126.7(\mathrm{Ar} \mathrm{CH}), 125.5(\mathrm{Ar} \mathrm{C})$, $124.7(\mathrm{Ar} \mathrm{CH}), 121.2(\mathrm{Ar} \mathrm{CH}), 61.0\left(\mathrm{OCH}_{2}\right), 21.7$ $(\mathrm{Ar}-\mathrm{Me}), 14.6\left(\mathrm{CH}_{3}\right)$. The observed characterisation data (IR, ${ }^{1} \mathrm{H}$ and $\left.{ }^{13} \mathrm{C} \mathrm{NMR}\right)$ was consistent with that previously reported in the literature. ${ }^{21}$

SMILES: $\mathrm{O}=\mathrm{C}(\mathrm{OCC}) \mathrm{C}(\mathrm{C} 1=\mathrm{CC}(\mathrm{C})=\mathrm{CC}=\mathrm{C} 1)=[\mathrm{N}+]=[\mathrm{N}-]$

InChl = 1S/C11H12N2O2/c1-3-15-11(14)10(13-12)9-6-4-5-8(2)7-9/h4-7H,3H2,1-2H3

\section{Ethyl (3-trifluoromethylphenyl)diazoacetate (49)}<smiles>CCOC(=O)C(=N)c1cccc(C(F)(F)F)c1</smiles>

DBU $(0.90 \mathrm{~mL}, 6.0 \mathrm{mmol} 1.2$ equiv) was added dropwise to a stirred solution of $\mathbf{S} 5$ (1.16 g $5.0 \mathrm{mmol}$, 1.0 equiv) and $p$-ABSA (1.44 g, $6.0 \mathrm{mmol}, 1.2$ equiv) in $\mathrm{MeCN}(10 \mathrm{~mL})$ at $0{ }^{\circ} \mathrm{C}$. The mixture was then warmed to $25^{\circ} \mathrm{C}$ and stirred for $24 \mathrm{~h}$. The reaction was quenched with saturated aq $\mathrm{NH}_{4} \mathrm{Cl}(30 \mathrm{~mL})$ and the mixture extracted with $\mathrm{Et}_{2} \mathrm{O}(3 \times 30 \mathrm{~mL})$. The organic extracts were washed with brine $(30 \mathrm{~mL})$, dried over sodium sulfate and filtered. The filtrate was concentrated in vacuo and purified by column chromatography using $5 \% \mathrm{Et}_{2} \mathrm{O}$ in pentane, affording $49(1.15 \mathrm{~g}, 89 \%)$ as a bright orange liquid. $\mathrm{R}_{f}=0.40\left(5 \% \mathrm{Et}_{2} \mathrm{O}\right.$ in pentane). IR (ATR)/cm ${ }^{-1}$ 2986, $2095(\mathrm{~N}=\mathrm{N}), 1696(\mathrm{C}=\mathrm{O}), 1603,1495,1454,1312,1282,1252,1152,1111$, 1033, 768, 678. ${ }^{1} \mathrm{H}$ NMR $\left(400 \mathrm{MHz}, \mathrm{CDCl}_{3}\right) \delta 7.80(\mathrm{~s}, 1 \mathrm{H}, \mathrm{Ar}-\mathrm{H}), 7.64(\mathrm{~d}, 1 \mathrm{H}, J=7.8 \mathrm{~Hz}, \mathrm{Ar}-\mathrm{H}), 7.49(\mathrm{t}, 1 \mathrm{H}$, $J=7.8 \mathrm{~Hz}, \mathrm{Ar}-\mathrm{H}), 7.42(\mathrm{~d}, 1 \mathrm{H}, J=7.8 \mathrm{~Hz}, \mathrm{Ar}-\mathrm{H}), 4.35\left(\mathrm{q}, 2 \mathrm{H}, J=7.1 \mathrm{~Hz}, \mathrm{OCH}_{2}\right), 1.36(\mathrm{t}, 3 \mathrm{H}, J=7.1 \mathrm{~Hz}$, $\left.\mathrm{CH}_{3}\right) .{ }^{13} \mathrm{C}$ NMR $\left(101 \mathrm{MHz}, \mathrm{CDCl}_{3}\right) \delta 164.7(\mathrm{C}=\mathrm{O}), 131.6\left(\mathrm{q}, \mathrm{J}_{\mathrm{CF}}=32.1 \mathrm{~Hz}, \mathrm{C}_{\mathrm{q}}-\mathrm{CF}_{3}\right), 129.5(\mathrm{Ar} \mathrm{CH}), 127.4(\mathrm{Ar}$ $\left.\mathrm{C}_{\mathrm{q}}\right), 126.7(\mathrm{ArCH}), 124.1$ (q, J JF $\left.=272.6 \mathrm{~Hz}, \mathrm{CF}_{3}\right), 122.4\left(\mathrm{q}, \mathrm{J}_{\mathrm{CF}}=3.7 \mathrm{~Hz}, \mathrm{ArCH}\right), 120.6\left(\mathrm{q}, J_{\mathrm{CF}}=3.7 \mathrm{~Hz}, \mathrm{Ar}\right.$ $\mathrm{CH}), 61.5\left(\mathrm{OCH}_{2}\right), 14.6\left(\mathrm{CH}_{3}\right) .{ }^{19} \mathrm{~F}\left\{{ }^{1} \mathrm{H}\right\} \mathrm{NMR}\left(377 \mathrm{MHz}, \mathrm{CDCl}_{3}\right) \delta-62.9$. HRMS (TOF ESI+) $\mathrm{m} / z$ calculated for $\mathrm{C}_{13} \mathrm{H}_{13} \mathrm{~F}_{3} \mathrm{~N}_{3} \mathrm{O}_{2}{ }^{+}[\mathrm{M}+\mathrm{MeCN}+\mathrm{H}]$ 300.0954. Found: 300.0960 .

SMILES: $\mathrm{O}=\mathrm{C}(\mathrm{OCC}) \mathrm{C}(\mathrm{C} 1=\mathrm{CC}(\mathrm{C}(\mathrm{F})(\mathrm{F}) \mathrm{F})=\mathrm{CC}=\mathrm{C} 1)=[\mathrm{N}+]=[\mathrm{N}-]$

InChI=1S/C11H9F3N2O2/c1-2-18-10(17)9(16-15)7-4-3-5-8(6-7)11(12,13)14/h3-6H,2H2,1H3 


\section{Ethyl (2-methoxyphenyl)diazoacetate (50)}<smiles>CCOC(=O)C(=N)c1ccccc1OC</smiles>

DBU $(0.9 \mathrm{~mL}, 6.0 \mathrm{mmol}, 1.2$ equiv) was added dropwise to a stirred solution of ethyl (2-methoxyphenyl)acetate S6 (0.97 g, $5.0 \mathrm{mmol}, 1.0$ equiv) and p-ABSA (1.44 g, $6.0 \mathrm{mmol}, 1.2$ equiv) in $\operatorname{MeCN}(10 \mathrm{~mL})$ at $0{ }^{\circ} \mathrm{C}$. The mixture was then warmed to $25^{\circ} \mathrm{C}$ and stirred for $25 \mathrm{~h}$. The reaction was quenched with saturated aq $\mathrm{NH}_{4} \mathrm{Cl}(30 \mathrm{~mL})$ and the mixture extracted with $\mathrm{Et}_{2} \mathrm{O}(3 \times 30 \mathrm{~mL})$. The organic extracts were washed with brine $(30 \mathrm{~mL})$, dried over sodium sulfate and filtered. The filtrate was concentrated in vacuo and purified by column chromatography using $8 \% \mathrm{Et}_{2} \mathrm{O}$ in pentane, affording $\mathbf{5 0}$ $(0.38 \mathrm{~g}, 34 \%)$ as a bright yellow liquid. $\mathrm{R}_{f}=0.29\left(8 \% \mathrm{Et}_{2} \mathrm{O}\right.$ in pentane). IR (ATR)/cm ${ }^{-1} 2982,2087(\mathrm{~N}=\mathrm{N})$, 1692 (C=O), 1595, 1495, 1461, 1368, 1290, 1245, 1148, 1021, 746. ${ }^{1} \mathrm{H}$ NMR $\left(400 \mathrm{MHz}, \mathrm{CDCl}_{3}\right) \delta 7.56$ (dd, $1 \mathrm{H}, J=7.8,1.7 \mathrm{~Hz}, \mathrm{Ar}-\mathrm{H}), 7.24-7.28(\mathrm{~m}, 1 \mathrm{H}, \mathrm{Ar}-\mathrm{H}), 7.02(\mathrm{td}, 1 \mathrm{H}, J=7.7,1.2 \mathrm{~Hz}, \mathrm{Ar}-\mathrm{H}), 6.89(\mathrm{dd}, 1 \mathrm{H}$, $J=8.33,1.0 \mathrm{~Hz}, \mathrm{Ar}-\mathrm{H}), 4.30\left(\mathrm{q}, 2 \mathrm{H}, J=7.2 \mathrm{~Hz}, \mathrm{OCH}_{2}\right), 3.86(\mathrm{~s}, 3 \mathrm{H}, \mathrm{OMe}), 1.32\left(\mathrm{t}, 3 \mathrm{H}, J=7.2 \mathrm{~Hz}, \mathrm{CH}_{3}\right) .{ }^{13} \mathrm{C}$ $\operatorname{NMR}\left(101 \mathrm{MHz}, \mathrm{CDCl}_{3}\right) \delta 166.4(\mathrm{C}=\mathrm{O}), 155.6\left(\mathrm{Ar} \mathrm{C} \mathrm{q}^{-} \mathrm{OMe}\right), 130.3(\mathrm{Ar} \mathrm{CH}), 128.7(\mathrm{Ar} \mathrm{CH}), 121.3(\mathrm{Ar} \mathrm{CH})$, 114.0 $\left(\mathrm{Ar}-\mathrm{C}_{\mathrm{q}}\right), 111.0(\mathrm{Ar} \mathrm{CH}), 61.0\left(\mathrm{OCH}_{2}\right), 55.7(\mathrm{OMe}), 14.7\left(\mathrm{CH}_{3}\right)$. The observed characterisation data (IR, ${ }^{1} \mathrm{H}$ and ${ }^{13} \mathrm{C}$ NMR) was consistent with that previously reported in the literature. ${ }^{43}$

SMILES: $\mathrm{O}=\mathrm{C}(\mathrm{OCC}) \mathrm{C}(\mathrm{C} 1=\mathrm{C}(\mathrm{OC}) \mathrm{C}=\mathrm{CC}=\mathrm{C} 1)=[\mathrm{N}+]=[\mathrm{N}-]$

InChI = 1S/C11H12N2O3/c1-3-16-11(14)10(13-12)8-6-4-5-7-9(8)15-2/h4-7H,3H2,1-2H3

\section{Ethyl (2-trifluoromethylphenyl)diazoacetate (53)}<smiles>CCOC(=O)C(=N)c1ccccc1C(F)(F)F</smiles>

DBU $(0.9 \mathrm{~mL}, \quad 6.0 \mathrm{mmol}, 1.2$ equiv) was added dropwise to a stirred solution of ethyl (2-trifluoromethylphenyl)acetate $\mathbf{S} 7$ (1.2 g, $5.0 \mathrm{mmol}, 1.0$ equiv) and $p$-ABSA (1.44 g, $6.0 \mathrm{mmol}, 1.2$ equiv) in $\mathrm{MeCN}(10 \mathrm{~mL})$ at $0^{\circ} \mathrm{C}$. The mixture was then warmed to $25^{\circ} \mathrm{C}$ and stirred for $25 \mathrm{~h}$. The reaction was quenched with saturated aq $\mathrm{NH}_{4} \mathrm{Cl}(30 \mathrm{~mL})$ and the mixture extracted with $\mathrm{Et}_{2} \mathrm{O}(3 \times 30 \mathrm{~mL})$. The organic extracts were washed with brine $(30 \mathrm{~mL})$, dried over sodium sulfate and filtered. The filtrate was concentrated in vacuo and purified by column chromatography using $8 \% \mathrm{Et}_{2} \mathrm{O}$ in pentane, affording 53 $(0.79 \mathrm{~g}, 62 \%)$ as a bright yellow-orange liquid. $\mathrm{R}_{f}=0.19\left(10 \% \mathrm{Et}_{2} \mathrm{O}\right.$ in pentane). IR $(\mathrm{ATR}) / \mathrm{cm}^{-1} 2986,2095$ $(\mathrm{N}=\mathrm{N}), 1696(\mathrm{C}=\mathrm{O}), 1603,1495,1454,1312,1282,1252,1152,1111,1033,768,678 .{ }^{1} \mathrm{H} \mathrm{NMR}(400 \mathrm{MHz}$, $\left.\mathrm{CDCl}_{3}\right) \delta 7.75(\mathrm{~d}, 1 \mathrm{H}, J=8.2 \mathrm{~Hz}, \mathrm{Ar}-\mathrm{H}), 7.63-7.56(\mathrm{~m}, 2 \mathrm{H}, 2 \times \mathrm{Ar}-\mathrm{H}), 7.50(\mathrm{t}, 1 \mathrm{H}, J=7.4 \mathrm{~Hz}, \mathrm{Ar}-\mathrm{H}), 4.29$ (q, $\left.2 \mathrm{H}, J=7.1 \mathrm{~Hz}, \mathrm{OCH}_{2}\right), 1.29\left(\mathrm{t}, 3 \mathrm{H}, J=7.2 \mathrm{~Hz}, \mathrm{CH}_{3}\right) .{ }^{13} \mathrm{C} \mathrm{NMR}\left(101 \mathrm{MHz}, \mathrm{CDCl}_{3}\right) \delta 166.0(\mathrm{C}=\mathrm{O}), 134.5$ ( $\mathrm{Ar} \mathrm{CH}$ ), $132.3(\mathrm{Ar} \mathrm{CH}), 129.5$ (q, J JF $=30.2 \mathrm{~Hz}, \mathrm{C}_{\mathrm{q}}-\mathrm{CF}_{3}$ ), $129.4(\mathrm{Ar} \mathrm{CH}), 127.0$ (q, J $\left.\mathrm{CF}=5.3 \mathrm{~Hz}, \mathrm{CF}_{3}\right), 123.9$ (q, $\left.J_{\mathrm{CF}}=273.4 \mathrm{~Hz}, \mathrm{Ar} \mathrm{CH}\right), 123.8\left(\mathrm{Ar} \mathrm{C}_{\mathrm{q}}\right), 61.5\left(\mathrm{OCH}_{2}\right), 14.6\left(\mathrm{CH}_{3}\right) .{ }^{19} \mathrm{~F}\left\{{ }^{1} \mathrm{H}\right\} \mathrm{NMR}\left(377 \mathrm{MHz}, \mathrm{CDCl}_{3}\right) \delta-61.6$. The observed characterisation data (IR, ${ }^{1} \mathrm{H}$ and ${ }^{13} \mathrm{C}$ NMR) was consistent with that previously reported in the literature. ${ }^{44}$

SMILES: $\mathrm{O}=\mathrm{C}(\mathrm{OCC}) \mathrm{C}(\mathrm{C} 1=\mathrm{C}(\mathrm{C}(\mathrm{F})(\mathrm{F}) \mathrm{F}) \mathrm{C}=\mathrm{CC}=\mathrm{C} 1)=[\mathrm{N}+]=[\mathrm{N}-]$

InChI = 1S/C11H9F3N2O2/c1-2-18-10(17)9(16-15)7-5-3-4-6-8(7)11(12,13)14/h3-6H,2H2,1H3 


\section{Ethyl (4-phenoxyphenyl)diazoacetate (58)}<smiles>CCOC(=O)C(=N)c1ccc(Oc2ccccc2)cc1</smiles>

DBU (0.36 mL, $2.4 \mathrm{mmol}, 1.2$ equiv) was added dropwise to a stirred solution of ethyl (4-phenoxyphenyl)acetate S8 (513 mg, $2.0 \mathrm{mmol}, 1.0$ equiv) and p-ABSA (577 mg, $2.4 \mathrm{mmol}, 1.2$ equiv) in $\operatorname{MeCN}(5 \mathrm{~mL})$ at $0{ }^{\circ} \mathrm{C}$. The mixture was then warmed to $25{ }^{\circ} \mathrm{C}$ and stirred for $24 \mathrm{~h}$. The reaction was quenched with saturated aq $\mathrm{NH}_{4} \mathrm{Cl}(20 \mathrm{~mL})$ and the mixture extracted with $\mathrm{Et}_{2} \mathrm{O}(3 \times 20 \mathrm{~mL})$. The organic extracts were washed with water $(2 \times 20 \mathrm{~mL})$ then brine $(20 \mathrm{~mL})$, dried over sodium sulfate and filtered. The filtrate was concentrated in vacuo and purified by column chromatography using $5 \% \mathrm{Et}_{2} \mathrm{O}$ in pentane, affording $58(439 \mathrm{mg}, 78 \%)$ as a bright orange solid. $\mathrm{R}_{f}=0.34\left(5 \% \mathrm{Et}_{2} \mathrm{O}\right.$ in pentane). $\mathrm{mp}$ (DSC peak) = $42{ }^{\circ} \mathrm{C}$. IR (ATR) $/ \mathrm{cm}^{-1}$ 2982, $2076(\mathrm{~N}=\mathrm{N}), 1689(\mathrm{C}=\mathrm{O}), 1588,1476,1372,1334,1230,1152,1036,846,738$. ${ }^{1} \mathrm{H}$ NMR $\left(400 \mathrm{MHz}, \mathrm{CDCl}_{3}\right) \delta$ 7.46-7.41 (m, 2H, $\left.2 \times \mathrm{Ar}-\mathrm{H}\right), 7.37-7.31(\mathrm{~m}, 2 \mathrm{H}, 2 \times \mathrm{Ar}-\mathrm{H}), 7.13-7.09(\mathrm{~m}, 1 \mathrm{H}$, $\mathrm{Ar}-\mathrm{H}), 7.07-6.99(\mathrm{~m}, 4 \mathrm{H}, 4 \times \mathrm{Ar}-\mathrm{H}), 4.33\left(\mathrm{q}, 2 \mathrm{H}, J=7.1 \mathrm{~Hz}, \mathrm{OCH}_{2}\right), 1.34\left(\mathrm{t}, 3 \mathrm{H}, J=7.1 \mathrm{~Hz}, \mathrm{CH}_{3}\right) .{ }^{13} \mathrm{C} \mathrm{NMR}$ $\left(101 \mathrm{MHz}, \mathrm{CDCl}_{3}\right) \delta 165.6(\mathrm{C}=\mathrm{O}), 157.2\left(\mathrm{Ar} \mathrm{C}_{\mathrm{q}}-\mathrm{O}\right), 155.6\left(\mathrm{Ar} \mathrm{C}_{\mathrm{q}}-\mathrm{O}\right), 129.9(2 \times \mathrm{Ar} \mathrm{CH}), 126.0(2 \times \mathrm{Ar} \mathrm{CH})$, 123.5 ( $\mathrm{Ar} \mathrm{CH}), 120.3\left(\mathrm{Ar} \mathrm{C}_{\mathrm{q}}\right), 119.7(2 \times \mathrm{Ar} \mathrm{CH}), 119.0(2 \times \mathrm{Ar} \mathrm{CH}), 61.2\left(\mathrm{OCH}_{2}\right), 14.7\left(\mathrm{CH}_{3}\right)$. HRMS (TOF ESI+) $\mathrm{m} / z$ calculated for $\mathrm{C}_{16} \mathrm{H}_{15} \mathrm{O}_{3}{ }^{+}\left[\mathrm{M}-\mathrm{N}_{2}+\mathrm{H}\right]$ 255.1021. Found: 296.1020.

SMILES: $\mathrm{O}=\mathrm{C}(\mathrm{OCC}) \mathrm{C}(\mathrm{C} 1=\mathrm{CC}=\mathrm{C}(\mathrm{OC} 2=\mathrm{CC}=\mathrm{CC}=\mathrm{C} 2) \mathrm{C}=\mathrm{C} 1)=[\mathrm{N}+]=[\mathrm{N}-]$

InChl = 1S/C16H14N2O3/c1-2-20-16(19)15(18-17)12-8-10-14(11-9-12)21-13-6-4-3-5-7-13/h3-11H,2H2,1H3

\section{Ethyl 2-([1,1'-biphenyl]-4-yl)-2-diazoacetate (59)}<smiles>CCOC(=O)C(=N)c1ccc(-c2ccccc2)cc1</smiles>

DBU $(0.36 \mathrm{~mL}, 2.4 \mathrm{mmol}, 1.2$ equiv) was added dropwise to a stirred solution of ethyl ([1,1'-biphenyl]-4yl)acetate S9 (481 mg, $2.0 \mathrm{mmol}, 1.0$ equiv) and p-ABSA (577 mg, $2.4 \mathrm{mmol}, 1.2$ equiv) in MeCN (5 mL) at $0{ }^{\circ} \mathrm{C}$. The mixture was then warmed to $25^{\circ} \mathrm{C}$ and stirred for $24 \mathrm{~h}$. The reaction was quenched with saturated aq $\mathrm{NH}_{4} \mathrm{Cl}(20 \mathrm{~mL})$ and the mixture extracted with $\mathrm{Et}_{2} \mathrm{O}(3 \times 20 \mathrm{~mL})$. The organic extracts were washed with water $(2 \times 20 \mathrm{~mL})$ then brine $(20 \mathrm{~mL})$, dried over sodium sulfate and filtered. The filtrate was concentrated in vacuo and purified by column chromatography using $10 \% \mathrm{Et}_{2} \mathrm{O}$ in pentane, affording $59(378 \mathrm{mg}, 71 \%)$ as a bright orange solid. $\mathrm{R}_{f}=0.25\left(10 \% \mathrm{Et}_{2} \mathrm{O}\right.$ in pentane). $\mathrm{mp}$ (DSC peak) $=94^{\circ} \mathrm{C}$. IR (ATR)/ $/ \mathrm{cm}^{-1} 2980,2084$ $(\mathrm{N}=\mathrm{N}), 1696(\mathrm{C}=\mathrm{O}), 1487,1372,1338,1238,1163,1047,1033,846,818,760,693 .{ }^{1} \mathrm{H}$ NMR $(400 \mathrm{MHz}$, $\left.\mathrm{CDCl}_{3}\right) \delta$ 7.65-7.54 (m, 6H, $\left.6 \times \mathrm{Ar}-\mathrm{H}\right), 7.44(\mathrm{t}, 2 \mathrm{H}, J=7.4 \mathrm{~Hz}, 2 \times \mathrm{Ar}-\mathrm{H}), 7.34(\mathrm{t}, 1 \mathrm{H}, J=7.4 \mathrm{~Hz}, \mathrm{Ar}-\mathrm{H})$, $4.36\left(\mathrm{q}, 2 \mathrm{H}, J=7.2 \mathrm{~Hz}, \mathrm{OCH}_{2}\right), 1.36$ (t, $\left.3 \mathrm{H}, J=7.2 \mathrm{~Hz}, \mathrm{CH}_{3}\right) .{ }^{13} \mathrm{C} \mathrm{NMR}\left(101 \mathrm{MHz}, \mathrm{CDCl}_{3}\right) \delta 165.4(\mathrm{C}=\mathrm{O})$, 140.1 ( $\left.\mathrm{Ar} \mathrm{C} \mathrm{C}_{\mathrm{q}}-\mathrm{Ph}\right), 138.7\left(\mathrm{Ar} \mathrm{C} \mathrm{q}_{\mathrm{q}}-\mathrm{Ph}\right), 129.0(2 \times \mathrm{Ar} \mathrm{CH}), 127.7(2 \times \mathrm{Ar} \mathrm{CH}), 127.5(\mathrm{Ar} \mathrm{CH}), 127.0(2 \times \mathrm{Ar}$ $\mathrm{CH}), 124.7\left(\mathrm{Ar} \mathrm{C}_{\mathrm{q}}\right), 124.4(2 \times \mathrm{Ar} \mathrm{CH}), 61.2\left(\mathrm{OCH}_{2}\right), 14.7\left(\mathrm{CH}_{3}\right)$. The observed characterisation data $\left(\mathrm{IR},{ }^{1} \mathrm{H}\right.$ and ${ }^{13} \mathrm{C}$ NMR) was consistent with that previously reported in the literature..$^{45}$

SMILES: $\mathrm{O}=\mathrm{C}(\mathrm{OCC}) \mathrm{C}(\mathrm{C} 1=\mathrm{CC}=\mathrm{C}(\mathrm{C} 2=\mathrm{CC}=\mathrm{CC}=\mathrm{C} 2) \mathrm{C}=\mathrm{C} 1)=[\mathrm{N}+]=[\mathrm{N}-]$

$\mathrm{InChI}=1 \mathrm{~S} / \mathrm{C} 16 \mathrm{H} 14 \mathrm{~N} 2 \mathrm{O} 2 / \mathrm{c} 1-2-20-16(19) 15(18-17) 14-10-8-13(9-11-14) 12-6-4-3-5-7-12 / \mathrm{h} 3-11 \mathrm{H}, 2 \mathrm{H} 2,1 \mathrm{H} 3$ 


\section{Ethyl (pyridin-4-yl)diazoacetate (60)}<smiles>CCOC(=O)C(=N)c1ccncc1</smiles>

DBU $(0.9 \mathrm{~mL}, 6.0 \mathrm{mmol}, 1.2$ equiv) was added dropwise to a stirred solution of ethyl (pyridin-4-yl)acetate (826 mg, $5.0 \mathrm{mmol}, 1.0$ equiv) and p-ABSA (1.44 g, $6.0 \mathrm{mmol}, 1.2$ equiv) in MeCN (15 mL) at $0{ }^{\circ} \mathrm{C}$. The mixture was then warmed to $25^{\circ} \mathrm{C}$ and stirred for $24 \mathrm{~h}$. The reaction was quenched with saturated aq $\mathrm{NH}_{4} \mathrm{Cl}$ $(30 \mathrm{~mL})$ and the mixture extracted with $\mathrm{Et}_{2} \mathrm{O}(3 \times 30 \mathrm{~mL})$. The organic extracts were combined and hexane $(70 \mathrm{~mL})$ was added. The mixture was filtered and precipitate washed with hexane $(50 \mathrm{~mL})$, dried over sodium sulfate and filtered. The filtrate was concentrated in vacuo and purified by column chromatography using $30 \% \mathrm{MeCN}$ in toluene, affording $60(829 \mathrm{mg}, 87 \%)$ as a bright yellow solid. $\mathrm{R}_{f}=0.27(30 \% \mathrm{MeCN}$ in toluene). IR (ATR)/cm ${ }^{-1}$ 2986, 2907, $2102(\mathrm{~N}=\mathrm{N}), 1696(\mathrm{C}=\mathrm{O}), 1584,1539,1495,1372,1245,1167,1047$, 865, 813, 738. ${ }^{1} \mathrm{H}$ NMR (400 MHz, $\left.\mathrm{CDCl}_{3}\right) \delta 8.53$ (dd, $\left.2 \mathrm{H}, J=4.6,1.6 \mathrm{~Hz}, 2 \times \mathrm{Ar}-\mathrm{H}\right), 7.42(\mathrm{dd}, 2 \mathrm{H}, J=4.6$, $1.6 \mathrm{~Hz}, 2 \times \mathrm{Ar}-\mathrm{H}), 4.36\left(\mathrm{q}, 2 \mathrm{H}, J=7.1 \mathrm{~Hz}, \mathrm{OCH}_{2}\right), 1.36\left(\mathrm{t}, 3 \mathrm{H}, J=7.1 \mathrm{~Hz}, \mathrm{CH}_{3}\right) .{ }^{13} \mathrm{C} \mathrm{NMR}\left(101 \mathrm{MHz}^{\mathrm{C}} \mathrm{CDCl}_{3}\right)$ $\delta 163.8(\mathrm{C}=\mathrm{O}), 150.1(2 \times \mathrm{Ar} \mathrm{CH}), 135.8\left(\mathrm{Ar} \mathrm{C}_{\mathrm{q}}\right), 117.4(2 \times \mathrm{Ar} \mathrm{CH}), 61.6\left(\mathrm{OCH}_{2}\right), 14.6\left(\mathrm{CH}_{3}\right)$. The observed characterisation data (IR, ${ }^{1} \mathrm{H}$ and ${ }^{13} \mathrm{C}$ NMR) was consistent with that previously reported in the literature. ${ }^{24}$

SMILES: $\mathrm{O}=\mathrm{C}(\mathrm{OCC}) \mathrm{C}(\mathrm{C} 1=\mathrm{CC}=\mathrm{NC}=\mathrm{C} 1)=[\mathrm{N}+]=[\mathrm{N}-]$

InChI = 1S/C9H9N3O2/c1-2-14-9(13)8(12-10)7-3-5-11-6-4-7/h3-6H,2H2,1H3

\section{Ethyl (pyridin-3-yl)diazoacetate (61)}<smiles>CCOC(=O)C(=N)c1cccnc1</smiles>

DBU ( $0.45 \mathrm{~mL}, 3.0 \mathrm{mmol}, 1.2$ equiv) was added dropwise to a stirred solution of ethyl (pyridine-3-yl)acetate (413 mg, $2.5 \mathrm{mmol}, 1.0$ equiv) and p-ABSA (721 mg, $3.0 \mathrm{mmol}, 1.2$ equiv) in MeCN (6 mL) at $0^{\circ} \mathrm{C}$. The mixture was then warmed to $25^{\circ} \mathrm{C}$ and stirred for $24 \mathrm{~h}$. The reaction was quenched with saturated aq $\mathrm{NH}_{4} \mathrm{Cl}$ $(30 \mathrm{~mL})$ and the mixture extracted with $\mathrm{Et}_{2} \mathrm{O}(3 \times 30 \mathrm{~mL})$. The organic extracts were washed with brine $(30$ $\mathrm{mL}$ ), dried over sodium sulfate and filtered. The filtrate was concentrated in vacuo and purified by column chromatography using 20\% MeCN in toluene, affording 61 (437 mg, 91\%) as a bright yellow solid. This compound was liquid at $25^{\circ} \mathrm{C} . \mathrm{R}_{f}=0.29\left(20 \% \mathrm{MeCN}\right.$ in toluene). IR (ATR)/cm ${ }^{-1} 2982,2084(\mathrm{~N}=\mathrm{N}), 1696$ $(\mathrm{C}=\mathrm{O}), 1484,1420,1372,1252,1156,1059,801,738,705 .{ }^{1} \mathrm{H}$ NMR $\left(400 \mathrm{MHz}, \mathrm{CDCl}_{3}\right) \delta 8.68(\mathrm{dd}, 1 \mathrm{H}$, $\mathrm{J}=2.5,0.8 \mathrm{~Hz}, \mathrm{Ar}-\mathrm{H}), 8.41(\mathrm{dd}, 1 \mathrm{H}, \mathrm{J}=4.8,1.6 \mathrm{~Hz}, \mathrm{Ar}-\mathrm{H}), 7.87(\mathrm{dq}, 1 \mathrm{H}, \mathrm{J}=8.2,1.6 \mathrm{~Hz}, \mathrm{Ar}-\mathrm{H}), 7.29$ (ddd, $1 \mathrm{H}, \mathrm{J}=4.8,3.4,0.8, \mathrm{Ar}-\mathrm{H}), 4.34\left(\mathrm{q}, 2 \mathrm{H}, \mathrm{J}=7.1 \mathrm{~Hz}, \mathrm{OCH}_{2}\right), 1.34\left(\mathrm{t}, 3 \mathrm{H}, \mathrm{J}=7.1 \mathrm{~Hz}, \mathrm{CH}_{3}\right) .{ }^{13} \mathrm{C} \mathrm{NMR}^{\mathrm{N}}$ $(101 \mathrm{MHz}) \delta 164.7(\mathrm{C}=\mathrm{O}), 146.9(\mathrm{Ar} \mathrm{CH}), 145.0(\mathrm{Ar} \mathrm{CH}), 131.3(\mathrm{Ar} \mathrm{CH}), 123.6(\mathrm{Ar} \mathrm{CH}), 122.8\left(\operatorname{Ar~} \mathrm{C}_{\mathrm{q}}\right), 61.5$ $\left(\mathrm{OCH}_{2}\right), 14.6\left(\mathrm{CH}_{3}\right)$. The observed characterisation data $\left(\mathrm{IR},{ }^{1} \mathrm{H}\right.$ and $\left.{ }^{13} \mathrm{C} \mathrm{NMR}\right)$ are consistent with that previously reported in the literature. ${ }^{46}$

SMILES: $\mathrm{O}=\mathrm{C}(\mathrm{OCC}) \mathrm{C}(\mathrm{C} 1=\mathrm{CN}=\mathrm{CC}=\mathrm{C} 1)=[\mathrm{N}+]=[\mathrm{N}-]$

InChI = 1S/C9H9N3O2/c1-2-14-9(13)8(12-10)7-4-3-5-11-6-7/h3-6H,2H2,1H3 


\section{Ethyl $[1,2,3]$ triazolo[1,5-a]pyridine-3-carboxylate $(63)$}<smiles>CCOC(=O)c1nnn2ccccc12</smiles>

DBU ( $0.45 \mathrm{~mL}, 3.0 \mathrm{mmol}, 1.2$ equiv) was added dropwise to a stirred solution of ethyl (pyridine-2-yl)acetate (413 mg, $2.5 \mathrm{mmol}, 1.0$ equiv) and p-ABSA (721 mg, $3.0 \mathrm{mmol}, 1.2$ equiv) in $\mathrm{MeCN}(6 \mathrm{~mL})$ at $0{ }^{\circ} \mathrm{C}$. The mixture was then warmed to $25^{\circ} \mathrm{C}$ and stirred for $24 \mathrm{~h}$. The reaction was quenched with saturated aq $\mathrm{NH}_{4} \mathrm{Cl}$ $(30 \mathrm{~mL})$ and the mixture extracted with $\mathrm{Et}_{2} \mathrm{O}(3 \times 30 \mathrm{~mL})$. The organic extracts were washed with brine $(30$ $\mathrm{mL}$ ), dried over sodium sulfate and filtered. The filtrate was concentrated in vacuo and purified by column chromatography using $20 \% \mathrm{MeCN}$ in toluene, affording $63(241 \mathrm{mg}, 50 \%)$ as a white solid. $\mathrm{R}_{f}=0.42(20 \%$ MeCN in toluene). $\mathrm{mp}(\mathrm{DSC}$ peak $)=111^{\circ} \mathrm{C}$. IR $(\mathrm{ATR}) / \mathrm{cm}^{-1}$ 3094, 2982, $1696(\mathrm{C}=\mathrm{O})$, 1636, 1521, 1439, 1267, 1215, 1156, 1066, 787, 678. ${ }^{1} \mathrm{H}$ NMR $\left(400 \mathrm{MHz}, \mathrm{CDCl}_{3}\right) \delta 8.84$ (dt, $\left.1 \mathrm{H}, J=7.0,1.0 \mathrm{~Hz}, \mathrm{Ar}-\mathrm{H}\right), 8.28(\mathrm{dt}$, $1 \mathrm{H}, J=8.9,1.2 \mathrm{~Hz}, \mathrm{Ar}-\mathrm{H}), 7.55$ (ddd, $1 \mathrm{H}, J=8.9,6.9,1.0 \mathrm{~Hz}, \mathrm{Ar}-\mathrm{H}), 7.16(\mathrm{dt}, 1 \mathrm{H}, J=6.9,1.2 \mathrm{~Hz}, \mathrm{Ar}-\mathrm{H})$, $4.52\left(\mathrm{q}, 2 \mathrm{H}, J=7.2 \mathrm{~Hz}, \mathrm{OCH}_{2}\right), 1.48\left(\mathrm{t}, 3 \mathrm{H}, J=7.2 \mathrm{~Hz}, \mathrm{CH}_{3}\right) .{ }^{13} \mathrm{C}$ NMR $(101 \mathrm{MHz}) \delta 161.6(\mathrm{C}=\mathrm{O}), 135.3(\mathrm{Ar}$ $\left.\mathrm{C}_{\mathrm{q}}\right), 129.7(\mathrm{Ar} \mathrm{C}$ ) 129.2 ( $\mathrm{Ar} \mathrm{CH}), 126.1(\mathrm{Ar} \mathrm{CH}), 119.6(\mathrm{Ar} \mathrm{CH}), 116.5(\mathrm{Ar} \mathrm{CH}), 61.3\left(\mathrm{OCH}_{2}\right), 14.6\left(\mathrm{CH}_{3}\right)$. The observed characterisation data (IR, ${ }^{1} \mathrm{H}$ and ${ }^{13} \mathrm{C}$ NMR) are consistent with that previously reported in the literature. ${ }^{43}$

SMILES: $\mathrm{O}=\mathrm{C}(\mathrm{OCC}) \mathrm{C} 1=\mathrm{C} 2 \mathrm{~N}(\mathrm{C}=\mathrm{CC}=\mathrm{C} 2) \mathrm{N}=\mathrm{N} 1$

InChl = 1S/C9H9N3O2/c1-2-14-9(13)8-7-5-3-4-6-12(7)11-10-8/h3-6H,2H2,1H3

The following diazo compound was synthesised using a procedure adapted from Chiara and Suárez: ${ }^{47}$

\section{Ethyl (nitro)diazoacetate (64)}<smiles>CCOC(=O)C(=[W])[N+](=O)[O-]</smiles>

2,6-Lutidine ( $0.41 \mathrm{~mL}, 3.5 \mathrm{mmol}, 1.4$ equiv) was added dropwise to a stirred solution of ethyl nitroacetate ( $0.28 \mathrm{~mL}, 2.5 \mathrm{mmol}, 1.0$ equiv) and $\mathrm{NfN}_{3}$ (894 mg, $3.0 \mathrm{mmol}, 1.2$ equiv) in $\mathrm{CH}_{2} \mathrm{Cl}_{2}(3 \mathrm{~mL})$ at $0{ }^{\circ} \mathrm{C}$. The mixture was then warmed to $25{ }^{\circ} \mathrm{C}$ and stirred for $24 \mathrm{~h}$. The reaction was quenched with saturated aq $\mathrm{NaHCO}_{3}(20 \mathrm{~mL})$ and the mixture extracted with $\mathrm{CH}_{2} \mathrm{Cl}_{2}(2 \times 25 \mathrm{~mL})$. The organic extracts were washed with saturated aq $\mathrm{NaHCO}_{3}(2 \times 20 \mathrm{~mL})$, dried over sodium sulfate and filtered. The filtrate was concentrated in vacuo and $\mathrm{CHCl}_{3}(40 \mathrm{~mL})$ was added before the solution was filtered over a plug of silica, which caused the brightly coloured diazo compound to remain on the silica. After washing with $\mathrm{CHCl}_{3}(100 \mathrm{~mL})$, the filtrate was discarded and the diazo compound eluted with MeCN. The MeCN filtrate was concentrated in vacuo afforded 64 (82 mg, 21\%) as a dark orange liquid. $\mathrm{R}_{f}=0.79\left(50 \% \mathrm{EtOAc}\right.$ in MeCN). IR (ATR)/cm ${ }^{-1} 2989$, $2147(\mathrm{~N}=\mathrm{N}), 1730(\mathrm{C}=\mathrm{O}), 1513,1320,1193,1111,1010,869,746 .{ }^{1} \mathrm{H} \mathrm{NMR}\left(400 \mathrm{MHz}, \mathrm{CDCl}_{3}\right) \delta 4.41(\mathrm{q}, 2 \mathrm{H}$, $\left.J=7.1 \mathrm{~Hz}, \mathrm{OCH}_{2}\right), 1.37\left(\mathrm{t}, 3 \mathrm{H}, J=7.1 \mathrm{~Hz}, \mathrm{CH}_{3}\right) \cdot{ }^{13} \mathrm{C} \mathrm{NMR}(101 \mathrm{MHz}) \delta 155.5(\mathrm{C}=\mathrm{O}), 63.3\left(\mathrm{OCH}_{2}\right), 14.4$ $\left(\mathrm{CH}_{3}\right)$. The observed characterisation data (IR, ${ }^{1} \mathrm{H}$ and $\left.{ }^{13} \mathrm{C} N \mathrm{NMR}\right)$ are consistent with that previously reported in the literature..$^{48,49}$

SMILES: $\mathrm{O}=\mathrm{C}(\mathrm{OCC}) \mathrm{C}([\mathrm{N}+]([\mathrm{O}-])=\mathrm{O})=[\mathrm{N}+]=[\mathrm{N}-]$

InChI = 1S/C4H5N3O4/c1-2-11-4(8)3(6-5)7(9)10/h2H2,1H3 
The following diazo compounds were synthesised using a procedure adapted from Gouverneur: ${ }^{12}$

\section{(1-Diazo)-2,2,2-trifluoroethyl)benzene (73)}<smiles>N#CC(F)(F)c1ccccc1</smiles>

$\mathrm{KOH}$ (988 mg, $17.6 \mathrm{mmol}, 2.0$ equiv) was added to a solution of 4-methyl- $N$-(2,2,2-trifluoro-1phenylethylidene)benzenesulfonohydrazide $\mathbf{S 1 0}(3.0 \mathrm{~g}, 8.8 \mathrm{mmol}, 1.0$ equiv) in methanol $(22 \mathrm{~mL})$. The mixture was heated to $65^{\circ} \mathrm{C}$ for $1 \mathrm{~h}$ and then cooled to rt. $\mathrm{H}_{2} \mathrm{O}(100 \mathrm{~mL})$ was added and the aqueous mixture was extracted with pentane $(3 \times 100 \mathrm{~mL})$. The combined organic layers were washed with saturated aq $\mathrm{NaHCO}_{3}(50 \mathrm{~mL})$, then brine $(50 \mathrm{~mL})$ and dried over sodium sulfate, filtered and concentrated in vacuo. Purification by flash chromatography using pentane, followed by distillation to remove residual pentane by gentle heating $\left(T<50{ }^{\circ} \mathrm{C}\right.$ ) afforded 73 as a volatile orange oil. (413 mg, 25\%). $\mathrm{R}_{f}=0.68$ (pentane). IR (film) $/ \mathrm{cm}^{-1} 2080$ (C=N=N in-phase), 1502, 1342 ( $\mathrm{C}=\mathrm{N}=\mathrm{N}$ out-of-phase), 1297, 1141, 1096, 954, 746, 686. ${ }^{1} \mathrm{H}$ NMR $\left(400 \mathrm{MHz}, \mathrm{CDCl}_{3}\right) \delta$ 7.45-7.39 (m, 2H, $\left.2 \times \mathrm{Ar}-\mathrm{H}\right), 7.25-7.20(\mathrm{~m}, 1 \mathrm{H}, \mathrm{Ar}-\mathrm{H}), 7.15-7.10(\mathrm{~m}, 2 \mathrm{H}, 2 \times$ $\mathrm{Ar}-\mathrm{H}) .{ }^{1} \mathrm{H}$ NMR $\left(400 \mathrm{MHz}, \mathrm{C}_{6} \mathrm{D}_{6}\right) \delta 6.99-6.93(\mathrm{~m}, 2 \mathrm{H}, 2 \times \mathrm{Ar}-\mathrm{H}), 6.85-6.80(\mathrm{~m}, 1 \mathrm{H}, \mathrm{Ar}-\mathrm{H}), 6.77-6.73(\mathrm{~m}$, $2 \mathrm{H}, 2 \times \mathrm{Ar}-\mathrm{H}) \cdot{ }^{13} \mathrm{C} \mathrm{NMR}\left(101 \mathrm{MHz}, \mathrm{CDCl}_{3}\right) \delta 129.4(2 \times \mathrm{Ar} \mathrm{CH}), 125.9(\mathrm{Ar} \mathrm{CH}), 125.6\left(\mathrm{q}, J_{\mathrm{CF}}=268.7 \mathrm{~Hz}\right.$, $\left.\mathrm{CF}_{3}\right), 123.5\left(\mathrm{Ar} \mathrm{C}_{\mathrm{q}}\right), 122.2(2 \times \mathrm{Ar} \mathrm{CH}) .{ }^{13} \mathrm{C} \mathrm{NMR}\left(101 \mathrm{MHz}, \mathrm{C}_{6} \mathrm{D}_{6}\right) \delta 130.1(2 \times \mathrm{Ar} \mathrm{CH}), 126.8\left(\mathrm{q}, \mathrm{J}_{\mathrm{CF}}=\right.$ $\left.269.8 \mathrm{~Hz}, \mathrm{CF}_{3}\right), 126.5(\mathrm{Ar} \mathrm{CH}), 124.1\left(\mathrm{Ar} \mathrm{C}\right.$ ), $123.0(2 \times \mathrm{Ar} \mathrm{CH}) .{ }^{19} \mathrm{~F}\left\{{ }^{1} \mathrm{H}\right\} \mathrm{NMR}\left(377 \mathrm{MHz}, \mathrm{CDCl}_{3}\right) \delta-57.4$. ${ }^{19} \mathrm{~F}\left\{{ }^{1} \mathrm{H}\right\}$ NMR $\left(377 \mathrm{MHz}, \mathrm{C}_{6} \mathrm{D}_{6}\right) \delta-57.6$. The observed characterisation data $\left({ }^{1} \mathrm{H} \mathrm{NMR}\right.$ in $\mathrm{CDCl}_{3},{ }^{13} \mathrm{C}$ and ${ }^{19} \mathrm{~F}$ NMR in $\mathrm{C}_{6} \mathrm{D}_{6}$ ) was consistent with that previously reported in the literature. ${ }^{50,12}$

SMILES: $\mathrm{FC}(\mathrm{C}(\mathrm{C} 1=\mathrm{CC}=\mathrm{CC}=\mathrm{C} 1)=[\mathrm{N}+]=[\mathrm{N}-])(\mathrm{F}) \mathrm{F}$

InChl = 1S/C8H5F3N2/c9-8(10,11)7(13-12)6-4-2-1-3-5-6/h1-5H

\section{1-Bromo-4-(1-diazo-2,2,2-trifluoroethyl)benzene (74)}<smiles>N#CC(F)(F)C(F)(F)F</smiles>

$\mathrm{KOH}$ (662 mg, $11.8 \mathrm{mmol}, 2.0$ equiv) was added to a solution of $\mathrm{N}^{\prime}$-(1-(4-bromophenyl)-2,2,2trifluoroethylidene)-4-methylbenzenesulfonohydrazide $S 11$ (2.50 g, $5.9 \mathrm{mmol}, 1.0 \mathrm{equiv})$ in methanol $(14.8 \mathrm{~mL})$. The mixture was heated to $70{ }^{\circ} \mathrm{C}$ for $1 \mathrm{~h}$ and then cooled to rt. $\mathrm{H}_{2} \mathrm{O}(100 \mathrm{~mL})$ was added and the aqueous mixture was extracted with $\mathrm{CH}_{2} \mathrm{Cl}_{2}(3 \times 100 \mathrm{~mL})$. The combined organic layers were washed with brine $(50 \mathrm{~mL})$, dried over sodium sulfate, filtered and concentrated in vacuo. Purification by flash chromatography using pentane afforded $74(738 \mathrm{mg}, 47 \%)$ as an orange oil. $\mathrm{R}_{f}=0.75$ (pentane). IR (film)/cm ${ }^{-1} 2080$ ( $\mathrm{C}=\mathrm{N}=\mathrm{N}$ out-of-phase), 1491, 1327 ( $\mathrm{C}=\mathrm{N}=\mathrm{N}$ in-phase), 1301, 1264, 1170, 1144, 1100, 954, 813. ${ }^{1} \mathrm{H}$ NMR $\left(400 \mathrm{MHz} \mathrm{CDCl}_{3}\right) \delta 7.52(\mathrm{~d}, 2 \mathrm{H}, \mathrm{J}=8.9 \mathrm{~Hz}, 2 \times \mathrm{Ar}-\mathrm{H}), 6.99-6.94(\mathrm{~m}, 2 \mathrm{H}, 2 \times \mathrm{Ar}-\mathrm{H}) .{ }^{13} \mathrm{C} \mathrm{NMR}$ $\left(101 \mathrm{MHz}, \mathrm{CDCl}_{3}\right) \delta 132.6$ and $132.5(2 \times \mathrm{Ar} \mathrm{CH}), 131.4\left(\mathrm{Ar} \mathrm{C}\right.$ ), $125.3\left(\mathrm{q}, \mathrm{J}_{\mathrm{CF}}=269.1 \mathrm{~Hz}, \mathrm{CF}_{3}\right), 123.6$ $(2 \times \mathrm{Ar} \mathrm{CH}), 122.7\left(\mathrm{C}=\mathrm{N}_{2}\right), 119.5\left(\mathrm{C}_{\mathrm{q}}-\mathrm{Br}\right) .{ }^{19} \mathrm{~F}\left\{{ }^{1} \mathrm{H}\right\} \quad \mathrm{NMR}\left(377 \mathrm{MHz}, \mathrm{CDCl}_{3}\right) \delta$-57.5. The observed characterisation data $\left({ }^{1} \mathrm{H},{ }^{13} \mathrm{C}\right.$ and ${ }^{19} \mathrm{~F}$ NMR $)$ was consistent with that previously reported in the literature. ${ }^{12}$

SMILES: $\mathrm{BrC} 1=\mathrm{CC}=\mathrm{C}(\mathrm{C}(\mathrm{C}(\mathrm{F})(\mathrm{F}) \mathrm{F})=[\mathrm{N}+]=[\mathrm{N}-]) \mathrm{C}=\mathrm{C} 1$

InChI = 1S/C8H4BrF3N2/c9-6-3-1-5(2-4-6)7(14-13)8(10,11)12/h1-4H 


\section{Azide reagents:}

The following diazo transfer reagents were prepared according to previous reports:

Tosyl azide $\left(\mathrm{TsN}_{3}\right)$ was synthesised using a procedure by Zhao. ${ }^{51}$

$\boldsymbol{p}$-CBSA and $\boldsymbol{m}$-CBSA were synthesised using the procedure by Collins and Maguire. ${ }^{52}$

o-NBSA was synthesised using a procedure by Du Bois. ${ }^{53}$

$\mathrm{NfN}_{3}$ was synthesised using a procedure by Zhu. ${ }^{54}$

Data for ADT is from our previous report ${ }^{55}$ and was synthesised using a procedure by Ma. ${ }^{56}$

\section{4-Carboxybenzenesulfonyl azide ( $p$-CBSA)}<smiles>[N+]S(=O)(=O)c1ccc(C(=O)O)cc1</smiles>

Sodium azide (130 mg, $2.0 \mathrm{mmol}, 1.0$ equiv) was dissolved in $5 \mathrm{~mL}$ of water and added dropwise to a stirred solution of 4-carboxybenzenesulfonyl chloride ( $441 \mathrm{mg}, 2.0 \mathrm{mmol}, 1.0$ equiv) in MeCN (7.5 mL). After $25 \mathrm{~min}$, the MeCN was removed in vacuo and the remaining aqueous solution was extracted with $\mathrm{Et}_{2} \mathrm{O}$ $(20 \mathrm{~mL})$. The organic phase was washed with water $(10 \mathrm{~mL})$, dried over sodium sulfate, filtered and concentrated in vacuo affording $p$-CBSA (359 mg, 81\%) as a white solid. $\mathrm{mp}$ (DSC peak) $=173{ }^{\circ} \mathrm{C}$. IR (ATR)/cm ${ }^{-1} 2140(\mathrm{~N}=\mathrm{N}=\mathrm{N}), 1689(\mathrm{C}=\mathrm{O}), 1424,1400,1372,1315,1163,760 .{ }^{1} \mathrm{H}$ NMR $\left(400 \mathrm{MHz}, \mathrm{MeOD}-d_{4}\right)$ $\delta 8.31(\mathrm{dt}, 2 \mathrm{H}, J=8.8,1.9 \mathrm{~Hz}, 2 \times \mathrm{Ar}-\mathrm{H}), 8.10(\mathrm{dt}, 2 \mathrm{H}, J=8.8,1.9 \mathrm{~Hz}, 2 \times \mathrm{Ar}-\mathrm{H}) .{ }^{13} \mathrm{C} \mathrm{NMR}(101 \mathrm{MHz}$, $\left.\mathrm{MeOD}-d_{4}\right) \delta 167.5(\mathrm{C}=\mathrm{O}), 143.3\left(\mathrm{Ar} \mathrm{C}_{\mathrm{q}}-\mathrm{SO}_{2}\right), 138.0\left(\mathrm{Ar} \mathrm{C}_{\mathrm{q}}-\mathrm{COOH}\right), 132.1(2 \times \operatorname{Ar} \mathrm{CH}), 128.7(2 \times \mathrm{Ar} \mathrm{CH})$. The observed characterisation data (IR, ${ }^{1} \mathrm{H}$ and ${ }^{13} \mathrm{C}$ NMR) are consistent with that previously reported in the literature..$^{57,58}$

SMILES: $\mathrm{O}=\mathrm{S}(\mathrm{C} 1=\mathrm{CC}=\mathrm{C}(\mathrm{C}(\mathrm{O})=\mathrm{O}) \mathrm{C}=\mathrm{C} 1)(\mathrm{N}=[\mathrm{N}+]=[\mathrm{N}-])=\mathrm{O}$

InChI = 1S/C7H5N3O4S/c8-9-10-15(13,14)6-3-1-5(2-4-6)7(11)12/h1-4H,(H,11,12)

\section{3-Carboxybenzenesulfonyl azide ( $m$-CBSA)}<smiles>NS(=O)(=O)c1cccc(C(=O)O)c1</smiles>

Sodium azide (130 mg, $2.0 \mathrm{mmol}, 1.0$ equiv) was dissolved in $5 \mathrm{~mL}$ of water and added dropwise to a stirred solution of 3-carboxybenzenesulfonyl chloride $(441 \mathrm{mg}, 2.0 \mathrm{mmol}, 1.0$ equiv) in MeCN (7.5 mL). After $25 \mathrm{~min}$, the MeCN was removed in vacuo and the remaining aqueous solution was extracted with $\mathrm{Et}_{2} \mathrm{O}$ $(20 \mathrm{~mL})$. The organic phase was washed with water $(10 \mathrm{~mL})$, dried over sodium sulfate, filtered and concentrated in vacuo affording $\boldsymbol{m}$-CBSA (336 mg, 76\%) as a white solid. $\mathrm{mp}$ (DSC peak) $=124^{\circ} \mathrm{C}$. IR (ATR)/cm ${ }^{-1} 2140(\mathrm{~N}=\mathrm{N}=\mathrm{N}), 1685(\mathrm{C}=\mathrm{O}), 1599,1413,1368,1290,1170,768 .{ }^{1} \mathrm{H}$ NMR $\left(400 \mathrm{MHz}, \mathrm{MeOD}-d_{4}\right)$ $\delta 8.53(\mathrm{~s}, 1 \mathrm{H}, \mathrm{Ar}-\mathrm{H}), 8.43(\mathrm{~d}, 1 \mathrm{H}, J=7.9 \mathrm{~Hz}, \mathrm{Ar}-\mathrm{H}), 8.21(\mathrm{~d}, 1 \mathrm{H}, J=7.9 \mathrm{~Hz}, A r-\mathrm{H}), 7.84(\mathrm{t}, 1 \mathrm{H}$, $J=7.9 \mathrm{~Hz}, \mathrm{Ar}-\mathrm{H}) .{ }^{13} \mathrm{C} \mathrm{NMR}\left(101 \mathrm{MHz}, \mathrm{MeOD}-d_{4}\right) \delta 167.2(\mathrm{C}=\mathrm{O}), 140.4\left(\mathrm{Ar} \mathrm{C}_{\mathrm{q}}-\mathrm{SO}_{2}\right), 136.8(\mathrm{Ar} \mathrm{CH}), 134.1$ $\left(\mathrm{Ar} \mathrm{C}_{\mathrm{q}}-\mathrm{COOH}\right), 132.4(\mathrm{Ar} \mathrm{CH}), 131.6(\mathrm{Ar} \mathrm{CH}), 129.4(\mathrm{Ar} \mathrm{CH})$. The observed characterisation data $\left(\mathrm{IR},{ }^{1} \mathrm{H}\right.$ and $\left.{ }^{13} \mathrm{C} N M R\right)$ are consistent with that previously reported in the literature..$^{52,59}$

SMILES: $\mathrm{O}=\mathrm{S}(\mathrm{C} 1=\mathrm{CC}=\mathrm{CC}(\mathrm{C}(\mathrm{O})=\mathrm{O})=\mathrm{C} 1)(\mathrm{N}=[\mathrm{N}+]=[\mathrm{N}-])=\mathrm{O}$

InChI = 1S/C7H5N3O4S/c8-9-10-15(13,14)6-3-1-2-5(4-6)7(11)12/h1-4H,(H,11,12) 


\section{2-Nitrobenzenesulfonyl azide (o-NBSA)}<smiles>O=[N+]([O-])c1ccccc1S(=O)(=O)[O-]</smiles>

o-Nitrobenzenesulfonyl chloride $(6.65 \mathrm{~g}, 30 \mathrm{mmol}, 1.0$ equiv) was dissolved in acetone $(14.6 \mathrm{~mL})$; the resulting solution was added to a stirred solution of sodium azide $(1.95 \mathrm{~g}, 33 \mathrm{mmol}, 1.1$ equiv) in acetone $(12.8 \mathrm{~mL})$ and water $(9.3 \mathrm{~mL})$ and stirred at $\mathrm{rt}$ for $2 \mathrm{~h}$. The acetone was removed in vacuo and the mixture extracted into $\mathrm{CH}_{2} \mathrm{Cl}_{2}(2 \times 50 \mathrm{~mL})$. The organic extracts were combined, washed with water $(25 \mathrm{~mL})$ then brine $(25 \mathrm{~mL})$, dried over $\mathrm{MgSO}_{4}$ and concentrated in vacuo affording o-NBSA $(6.51 \mathrm{~g}, 95 \%)$ as a yellow solid. $R_{f}=0.56\left(25 \%\right.$ hexane in $\left.\mathrm{CH}_{2} \mathrm{Cl}_{2}\right)$. IR (film)/cm ${ }^{-1} 3100,2337,2140(\mathrm{~N}=\mathrm{N}=\mathrm{N}), 1592,1541,1363,1307$, 1166, 1057, 853, 781, 752, 731, 653. ${ }^{1} \mathrm{H}$ NMR $\left(400 \mathrm{MHz}, \mathrm{CDCl}_{3}\right) \delta 8.17(\mathrm{~d}, 1 \mathrm{H}, \mathrm{J}=8 \mathrm{~Hz}, \mathrm{Ar}-\mathrm{H}), 7.95-7.83$ $(\mathrm{m}, 3 \mathrm{H}, 3 \times \mathrm{Ar}-\mathrm{H}) .{ }^{13} \mathrm{C} \mathrm{NMR}\left(101 \mathrm{MHz}, \mathrm{CDCl}_{3}\right) \delta 147.6\left(\mathrm{Ar} \mathrm{C}_{\mathrm{q}}-\mathrm{SO}_{2}\right), 136.1(\mathrm{Ar} \mathrm{CH}), 133.3(\mathrm{Ar} \mathrm{CH}), 132.2$ $\left(\mathrm{Ar} \mathrm{C}_{\mathrm{q}}-\mathrm{NO}_{2}\right), 131.6(\mathrm{Ar} \mathrm{CH}), 125.5(\mathrm{Ar} \mathrm{CH})$. The observed characterisation data (IR, ${ }^{1} \mathrm{H}$ and $\left.{ }^{13} \mathrm{C} \mathrm{NMR}\right)$ are consistent with that previously reported in the literature. ${ }^{53}$

SMILES: $\mathrm{O}=\mathrm{S}(\mathrm{C} 1=\mathrm{CC}=\mathrm{CC}=\mathrm{C} 1[\mathrm{~N}+]([\mathrm{O}-])=\mathrm{O})(\mathrm{N}=[\mathrm{N}+]=[\mathrm{N}-])=\mathrm{O}$

InChI = 1S/C6H4N4O4S/c7-8-9-15(13,14)6-4-2-1-3-5(6)10(11)12/h1-4H

\section{Nonafluorobutanesulfonyl azide $\left(\mathrm{NfN}_{3}\right)$}<smiles>O=S(=O)(O[Na])C(F)(F)C(F)(F)C(F)(F)C(F)(F)F</smiles>

Nonafluorobutanesulfonyl fluoride $(1.8 \mathrm{~mL}, 10 \mathrm{mmol}, 1.0$ equiv) was added dropwise to a stirred solution of sodium azide $\left(716 \mathrm{mg}, 11 \mathrm{mmol}, 1.1\right.$ equiv) in methanol $(20 \mathrm{~mL})$. The mixture was stirred at $25^{\circ} \mathrm{C}$ for $18 \mathrm{~h}$. The crude was added to $80 \mathrm{~mL}$ of ice-water in a separating funnel. The mixture was shaken and allowed to separate for $25 \mathrm{~min}$, then the bottom crude product layer was drained, dried over sodium sulfate and decanted giving neat $\mathbf{N f N}_{3}(1.86 \mathrm{~g}, 57 \%)$ as a clear faint yellow liquid. Caution $-\mathbf{N f N _ { 3 }}$ is semi-volatile (b.p. of

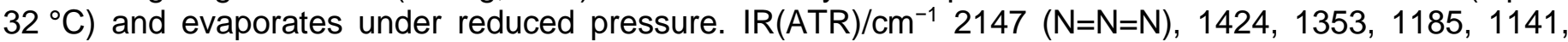
1029, 760. ${ }^{13} \mathrm{C} \mathrm{NMR}\left(101 \mathrm{MHz}, \mathrm{CDCl}_{3}\right) \delta 117.4$ (qt, $\left.\mathrm{J}_{\mathrm{CF}}=288,32.6 \mathrm{~Hz}, \mathrm{CF}_{3}\right) 115.6$ (tt, $J_{\mathrm{CF}}=303,36.8 \mathrm{~Hz}$, $\left.\mathrm{CF}_{2} \mathrm{SO}_{2}\right), 110.4\left(\mathrm{ttm}, \mathrm{J}_{\mathrm{CF}}=270,31.1 \mathrm{~Hz}, \mathrm{CF}_{2} \mathrm{CF}_{2} \mathrm{SO}_{2}\right), 108.7\left(\mathrm{tqm}, \mathrm{J}_{\mathrm{CF}}=270.9,40.1, \mathrm{CF}_{3} \mathrm{CF}_{2}\right) .{ }^{19} \mathrm{~F}\left\{{ }^{1} \mathrm{H}\right\} \mathrm{NMR}$ $\left(377 \mathrm{MHz}, \mathrm{CDCl}_{3}\right) \delta-81.6\left(\mathrm{CF}_{3}\right),-110.1\left(\mathrm{CF}_{2} \mathrm{SO}_{2}\right),-121.5\left(\mathrm{CF}_{2} \mathrm{CF}_{2} \mathrm{SO}_{2}\right),-126.6\left(\mathrm{CF}_{3} \mathrm{CF}_{2}\right)$. The observed characterisation data $\left({ }^{13} \mathrm{C}\right.$ and $\left.{ }^{19} \mathrm{~F} \mathrm{NMR}\right)$ are consistent with that previously reported in the literature. ${ }^{54,60}$

SMILES: $F C(C(F)(F) C(F)(S(=O)(N=[N+]=[N-])=O) F)(F) C(F)(F) F$

InChI = 1S/C4F9N3O2S/c5-1(6,3(9,10)11)2(7,8)4(12,13)19(17,18)16-15-14 


\section{Table S1. Hammett $\sigma$ Parameters Correlated with DSC Results}

Hammett substituent constants quoted from the survey by Hansch. ${ }^{61}$ Compounds are sorted by $\sigma$.

\begin{tabular}{|c|c|c|c|c|c|}
\hline entry & compound & $\mathrm{R}$ & $\sigma_{\mathrm{m}} / \sigma_{\mathrm{p}}{ }^{+}$ & $\begin{array}{l}\mathrm{T}_{\text {init }} \\
\left({ }^{\circ} \mathrm{C}\right)\end{array}$ & $\begin{array}{l}\text { Tonset } \\
\left({ }^{\circ} \mathrm{C}\right)\end{array}$ \\
\hline 1 & 38 & 4-OMe & -0.78 & 63 & 81 \\
\hline 2 & 39 & 4-Me & -0.31 & 79 & 103 \\
\hline 3 & 40 & $4-F$ & -0.07 & 72 & 101 \\
\hline 4 & 46 & 3-Me & -0.07 & 82 & 109 \\
\hline 5 & 9 & $\mathrm{H}$ & 0.00 & 87 & 109 \\
\hline 6 & 41 & $4-\mathrm{Cl}$ & 0.11 & 97 & 115 \\
\hline 7 & 45 & 3-OMe & 0.12 & 86 & 110 \\
\hline 8 & 42 & $4-\mathrm{Br}$ & 0.15 & 98 & 114 \\
\hline 9 & 47 & $3-F$ & 0.34 & 88 & 116 \\
\hline 10 & 48 & $3-\mathrm{Cl}$ & 0.37 & 95 & 117 \\
\hline 11 & 49 & $3-\mathrm{CF}_{3}$ & 0.43 & 92 & 117 \\
\hline 12 & 43 & $4-\mathrm{CF}_{3}$ & 0.61 & 96 & 123 \\
\hline 13 & 44 & $4-\mathrm{NO}_{2}$ & 0.79 & 113 & 131 \\
\hline 14 & 59 & 4-OPh & -0.50 & 81 & 95 \\
\hline 15 & 60 & 4-Ph & -0.18 & 80 & 108 \\
\hline
\end{tabular}




\section{DSC Plots of All Compounds}

The DSC experiment was repeated 6 times for ethyl (phenyl) diazoacetate 9 to establish repeatability. DSC for all other compounds exhibiting exothermic decomposition was conducted twice. The averaged DSC results in the manuscript used the results taken from each of the following plots. For compounds where DSC results were also obtained using the $2{ }^{\circ} \mathrm{C} \mathrm{min}^{-1}$ temperature ramp at GlaxoSmithKline (GSK), the plots are also given below.

Figure S1. DSC Plot for ethyl (phenyl)acetate (33)

Sample mass $-3.5 \mathrm{mg}$

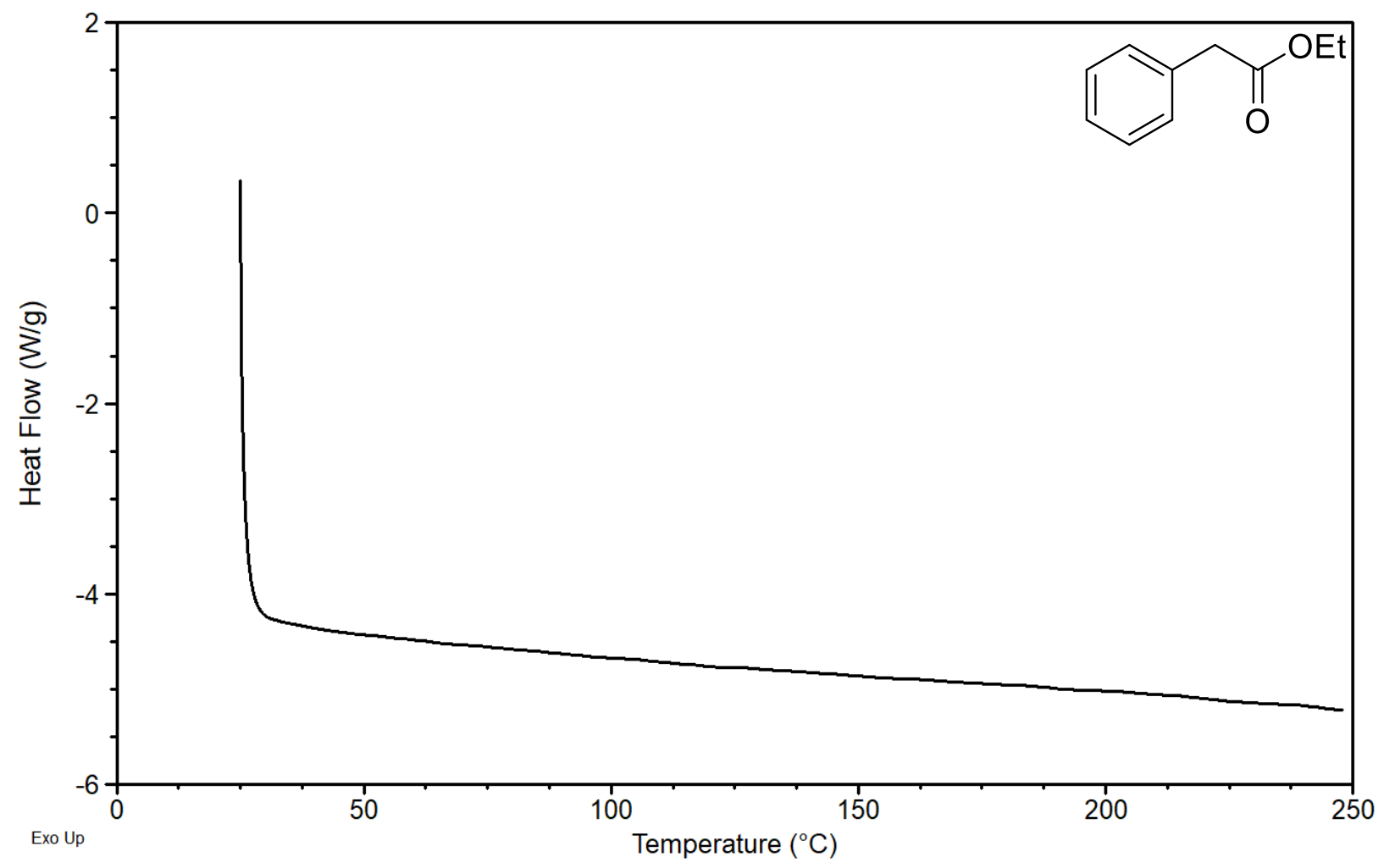


Figure S2. DSC Plot 1 for ethyl (phenyl)diazoacetate (9)

Sample mass $-4.1 \mathrm{mg}$

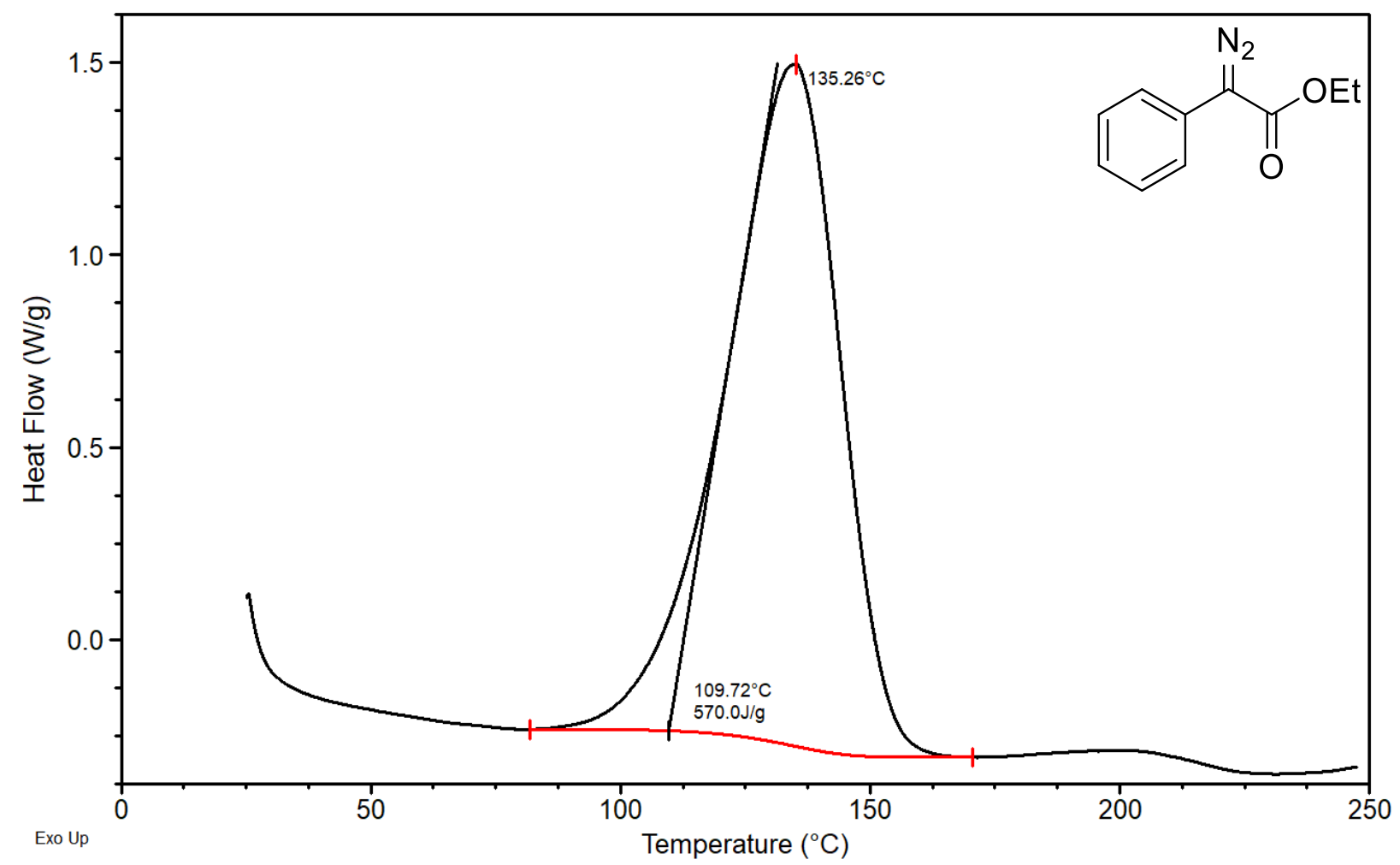

Figure S3. DSC Plot 2 for ethyl (phenyl)diazoacetate (9)

Sample mass $-4.0 \mathrm{mg}$

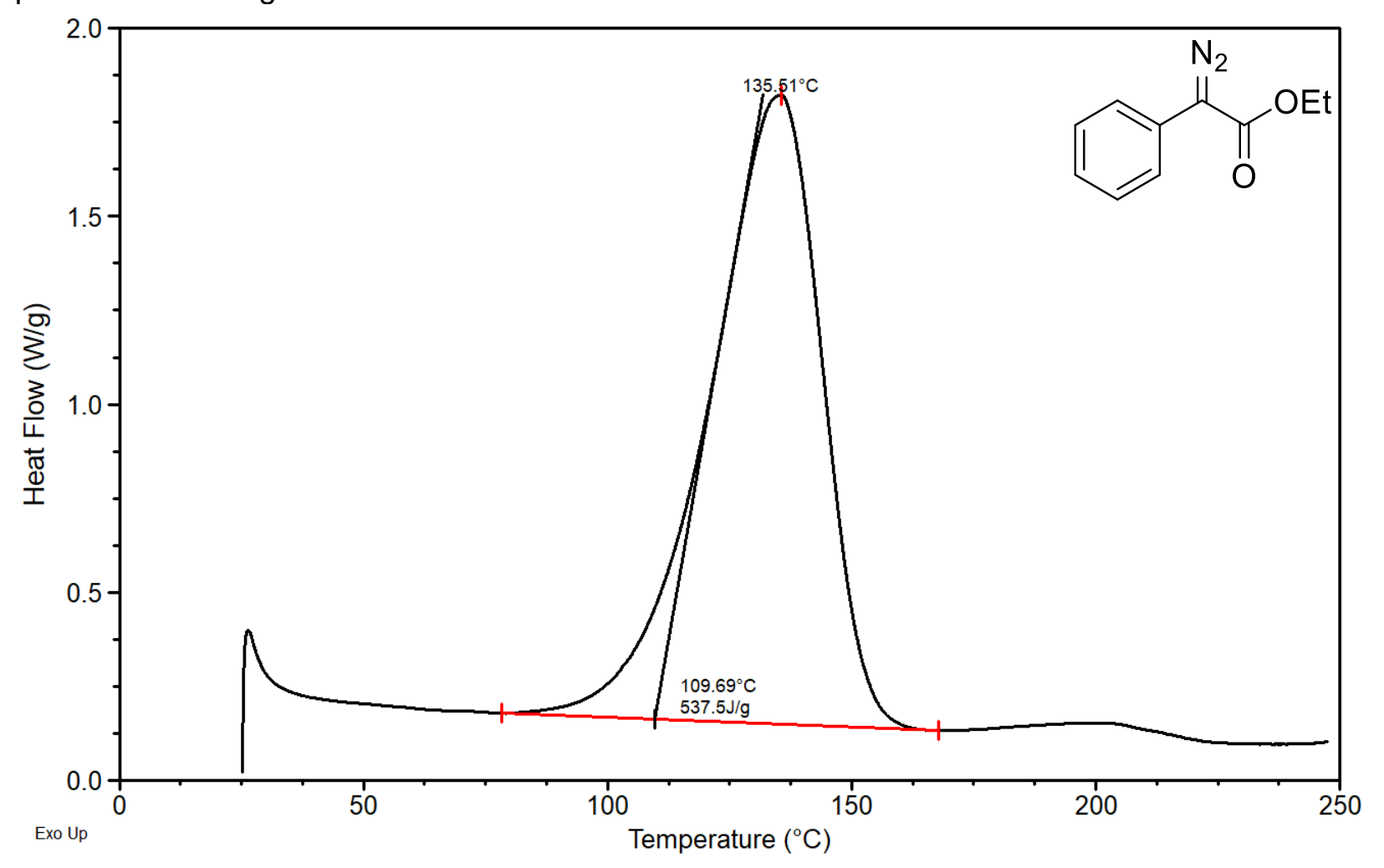


Figure S4. DSC Plot 3 for ethyl (phenyl)diazoacetate (9)

Sample mass $-4.9 \mathrm{mg}$

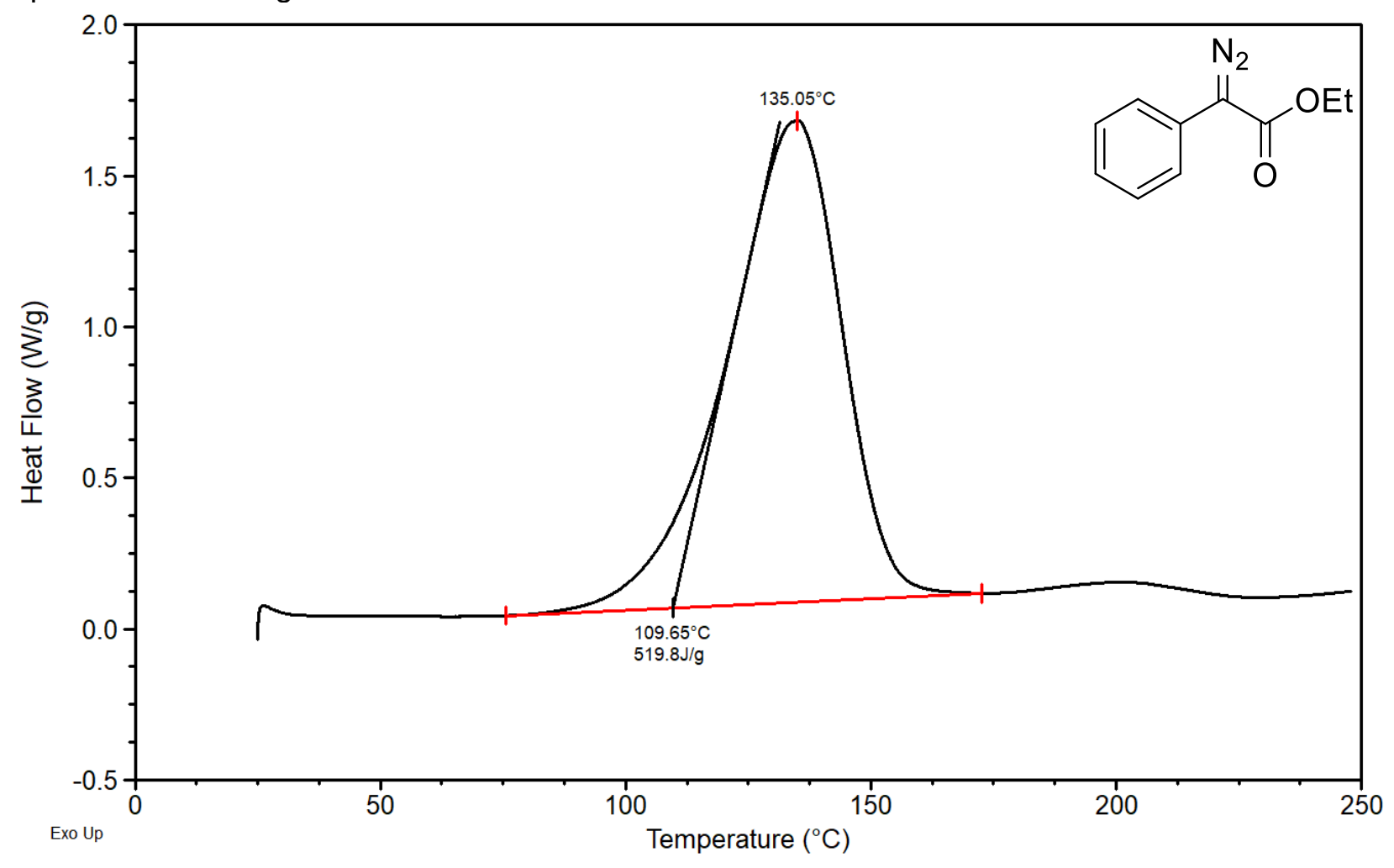

Figure S5. DSC Plot 4 for ethyl (phenyl)diazoacetate (9)

Sample mass $-5.5 \mathrm{mg}$

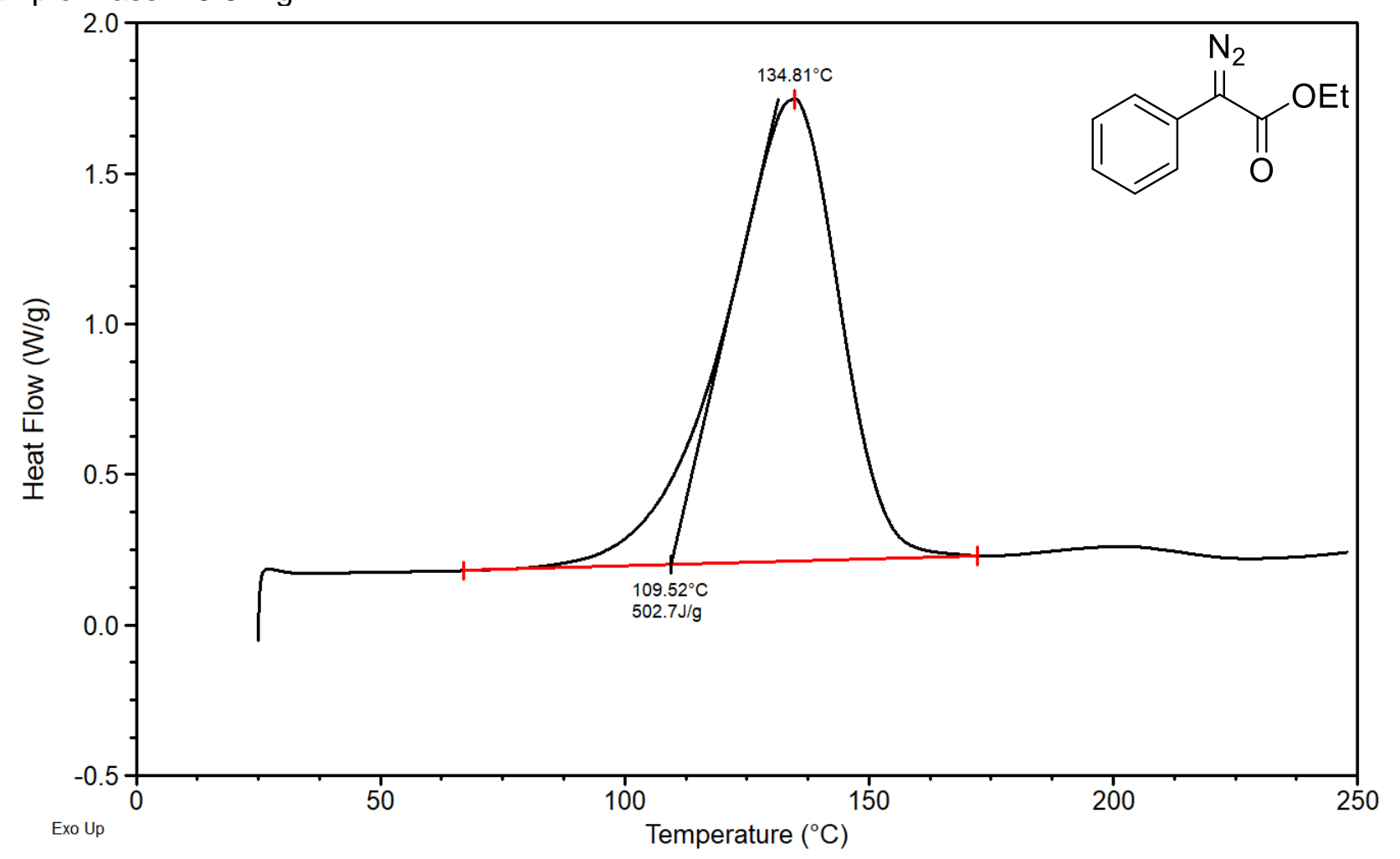


Figure S6. DSC Plot 5 for ethyl (phenyl)diazoacetate (9)

Sample mass $-2.1 \mathrm{mg}$

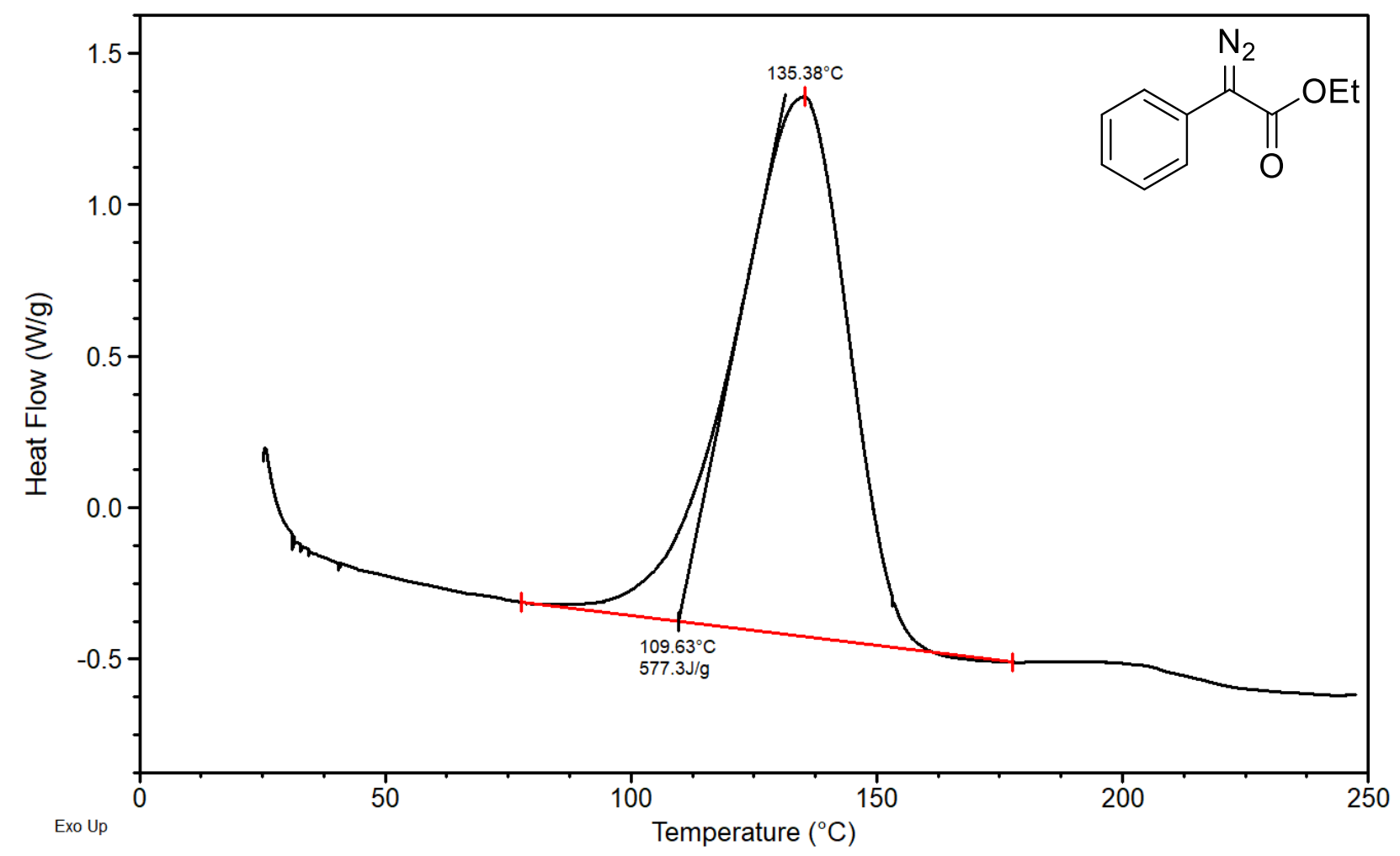

Figure S7. DSC Plot 6 for ethyl (phenyl)diazoacetate (9)

Sample mass $-5.0 \mathrm{mg}$

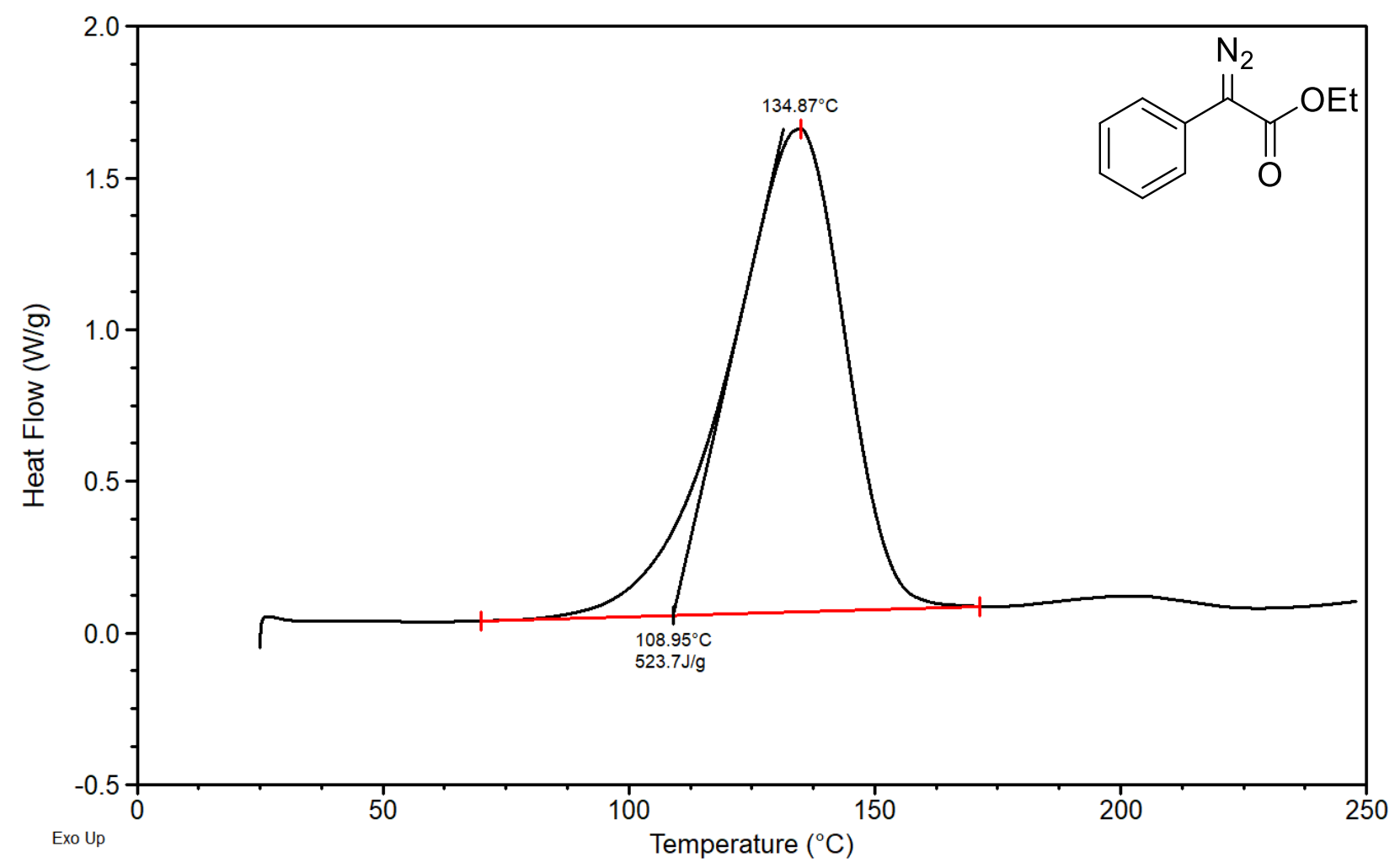


Figure S8. DSC Plot using Method B for ethyl (phenyl)diazoacetate (9)

Sample mass $-5.2 \mathrm{mg}$

^exo

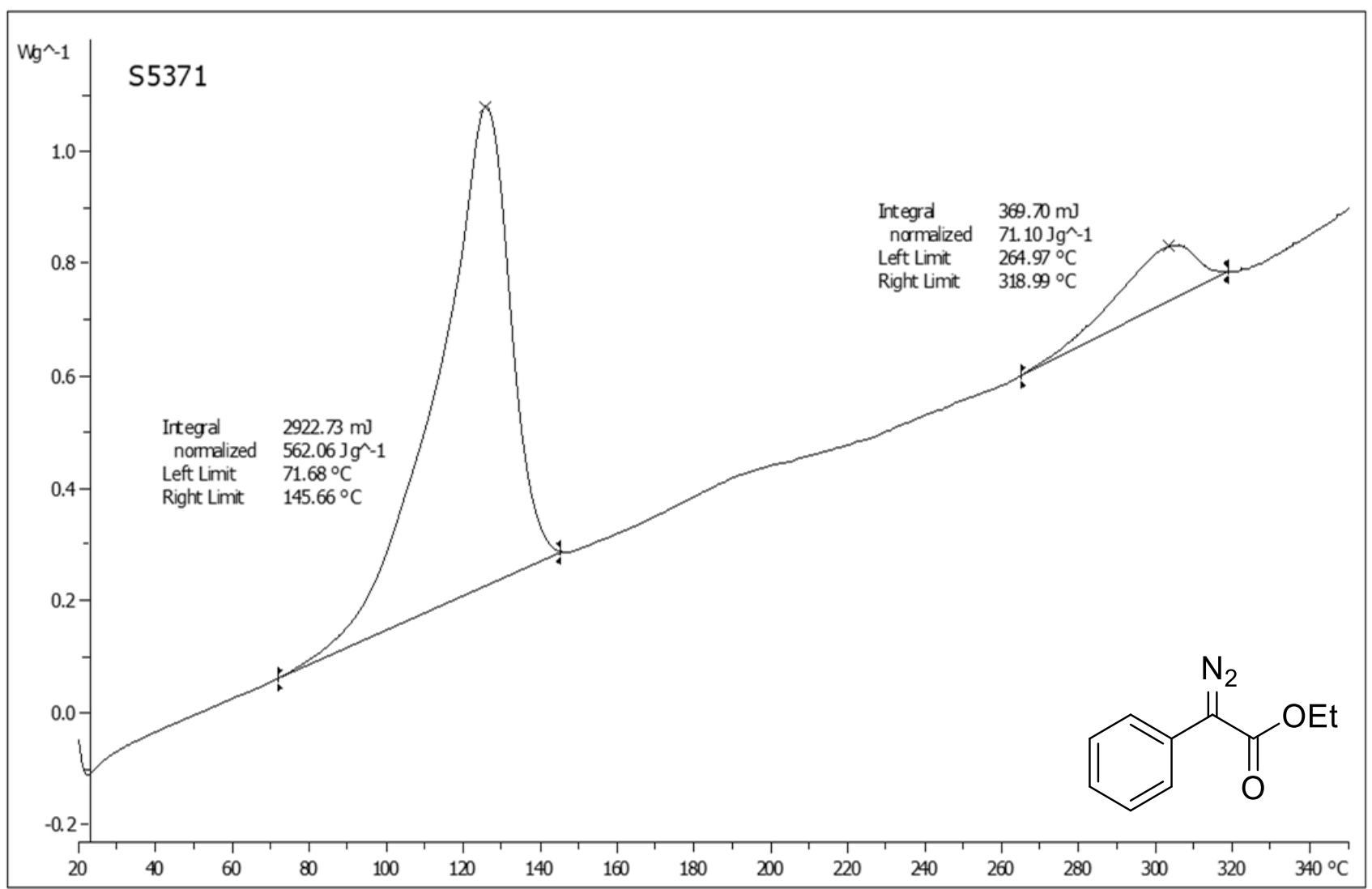


Figure S9. DSC Plot 1 for methyl (phenyl)diazoacetate (10)

Sample mass $-3.6 \mathrm{mg}$

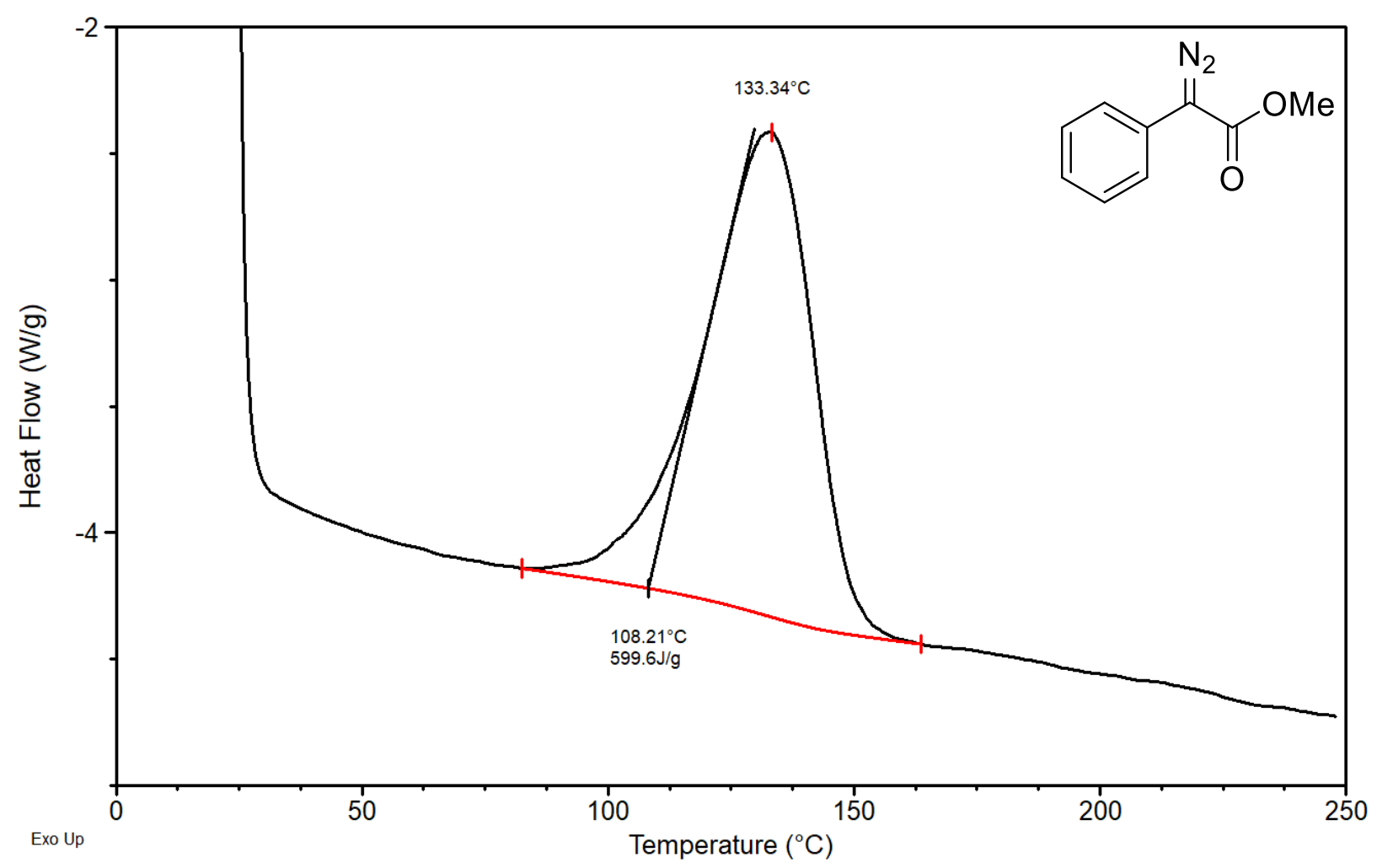

Figure S10. DSC Plot 2 for methyl (phenyl)diazoacetate (10)

Sample mass $-3.0 \mathrm{mg}$

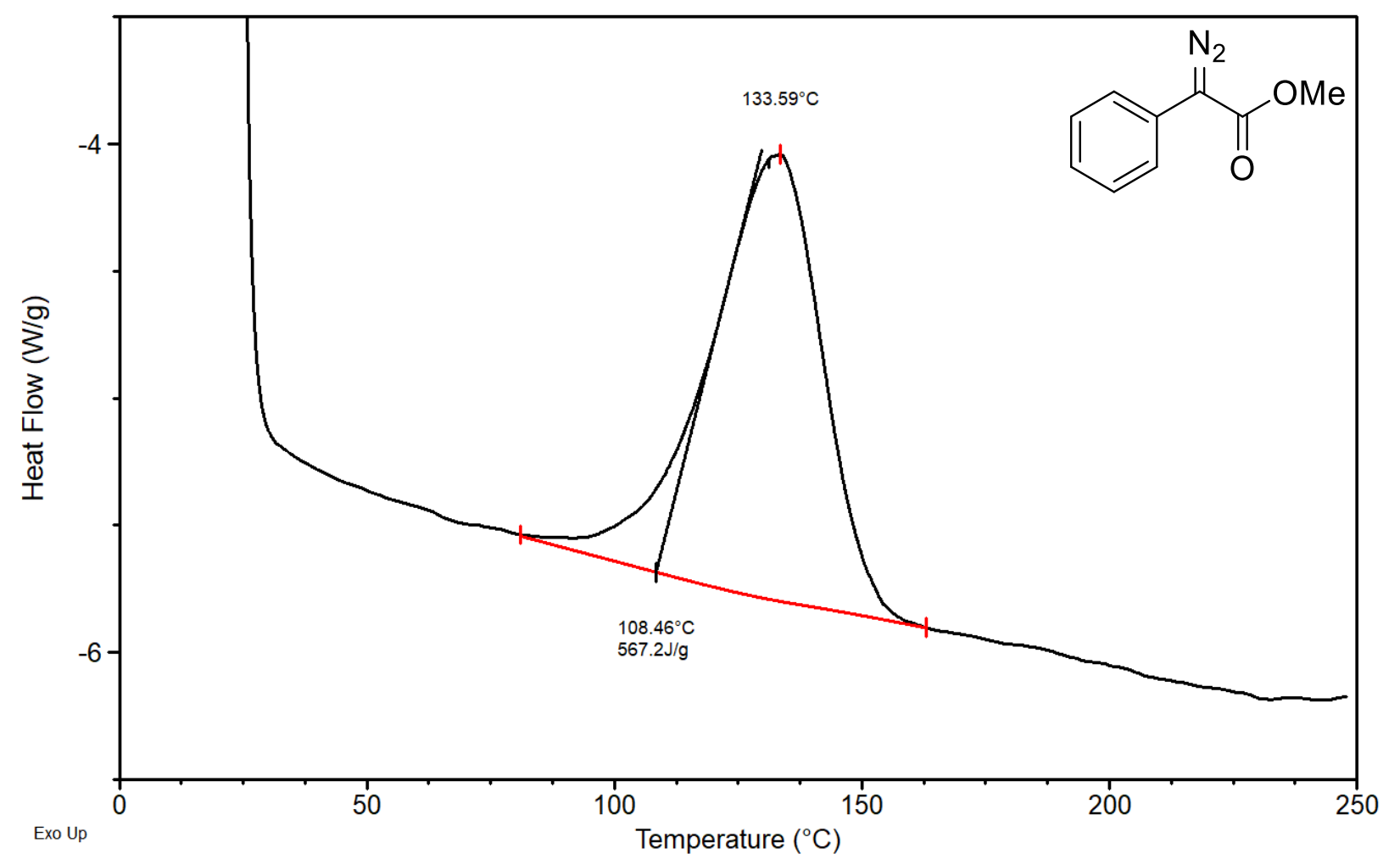


Figure S11. DSC Plot using Method B for methyl (phenyl)diazoacetate (10) Sample mass $-5.1 \mathrm{mg}$

^exo

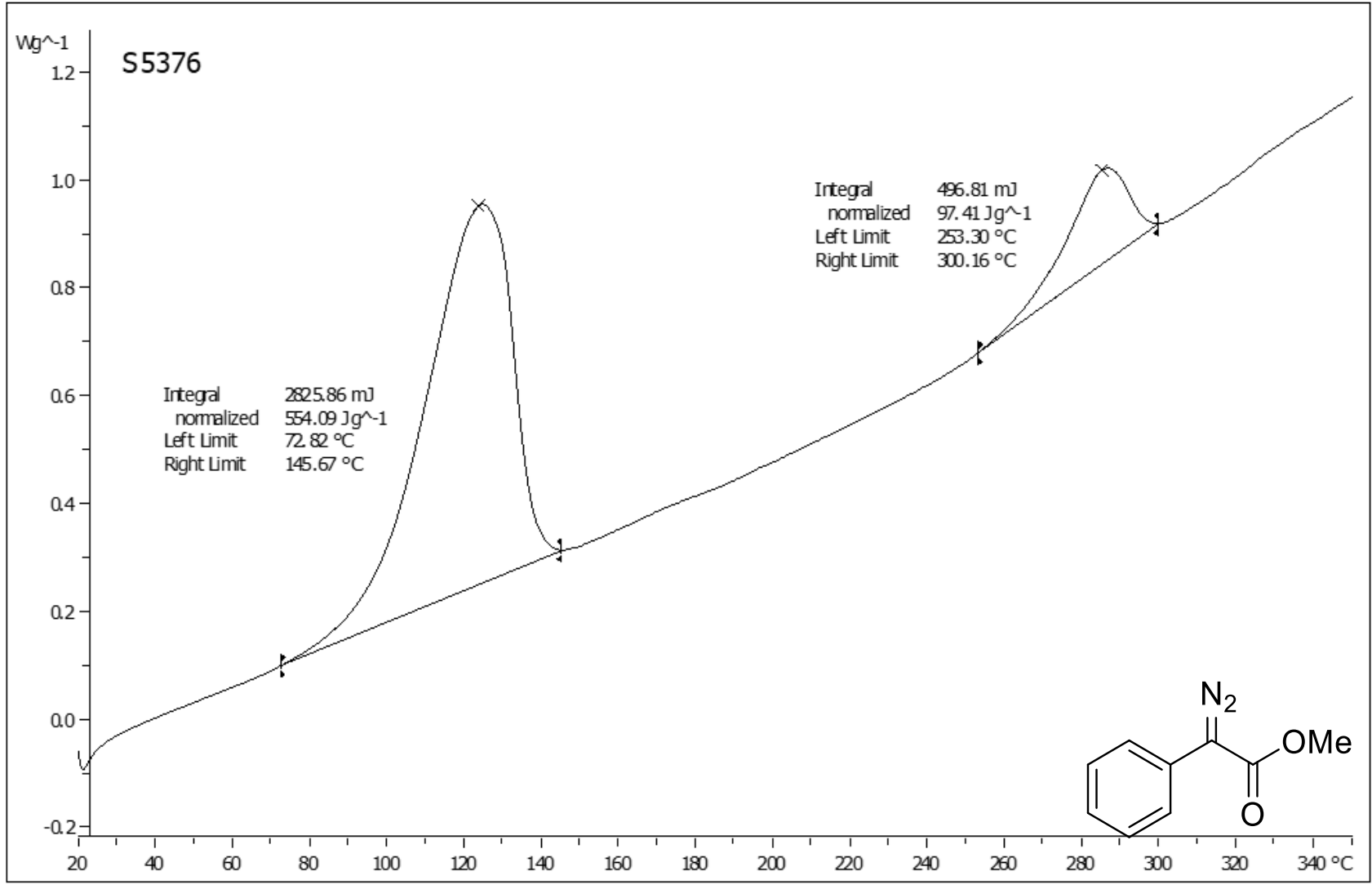


Figure S12. DSC Plot 1 for tert-butyl (phenyl)diazoacetate (34)

Sample mass $-2.5 \mathrm{mg}$

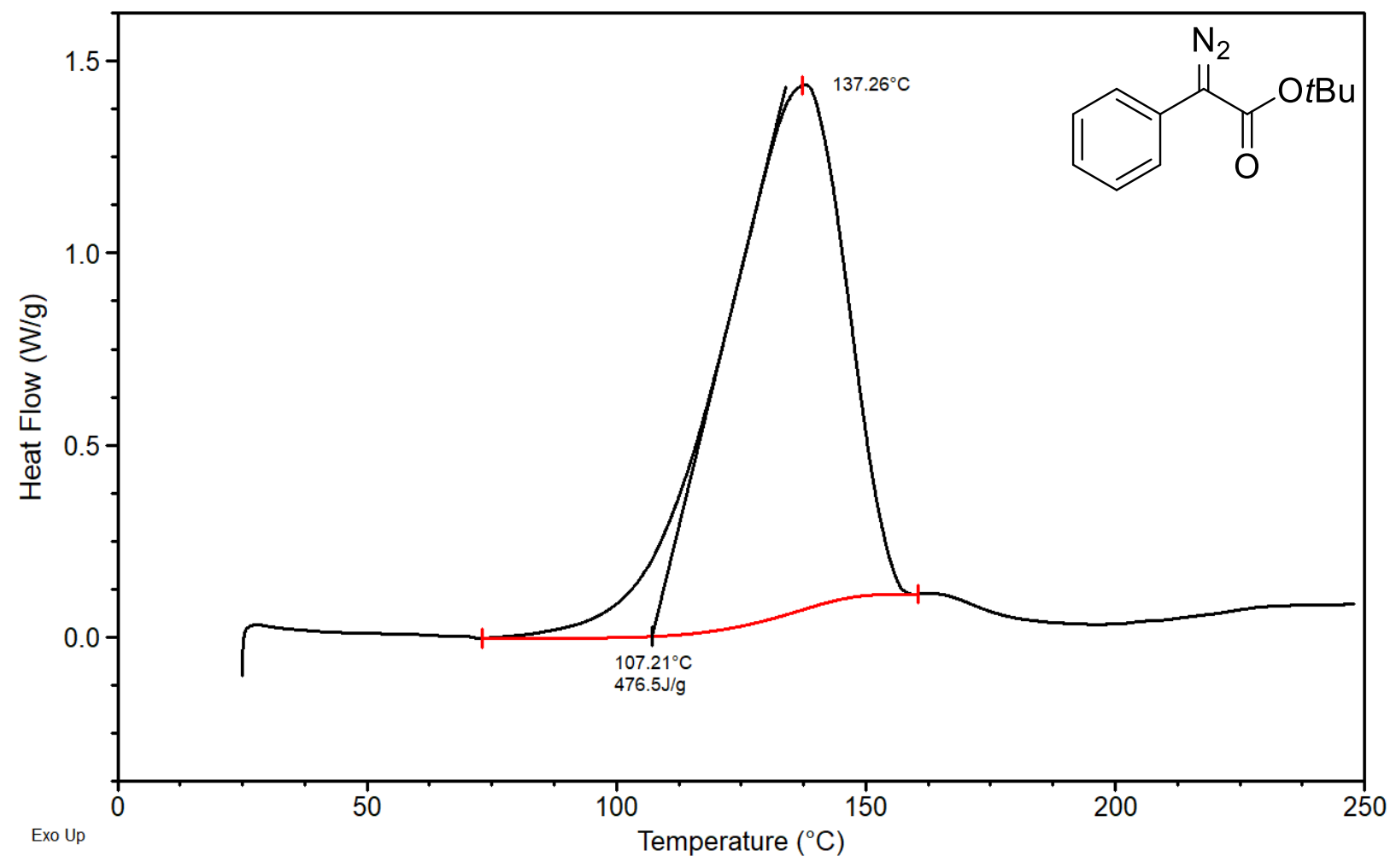

Figure S13. DSC Plot 2 for tert-butyl (phenyl)diazoacetate (34)

Sample mass $-3.9 \mathrm{mg}$

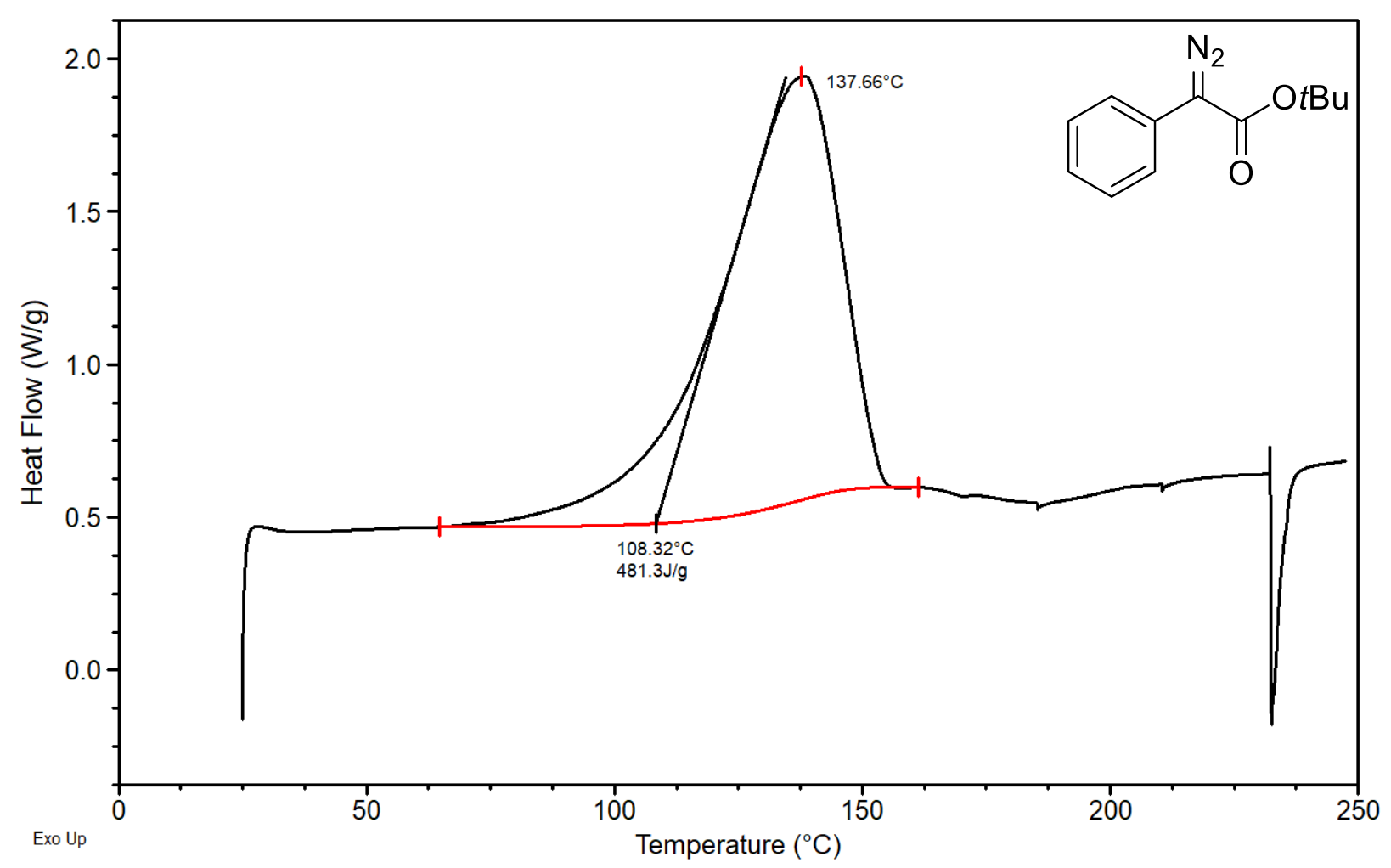


Figure S14. DSC Plot 1 for tert-butyl (4-bromophenyl)diazoacetate (35)

Sample mass $-5.9 \mathrm{mg}$

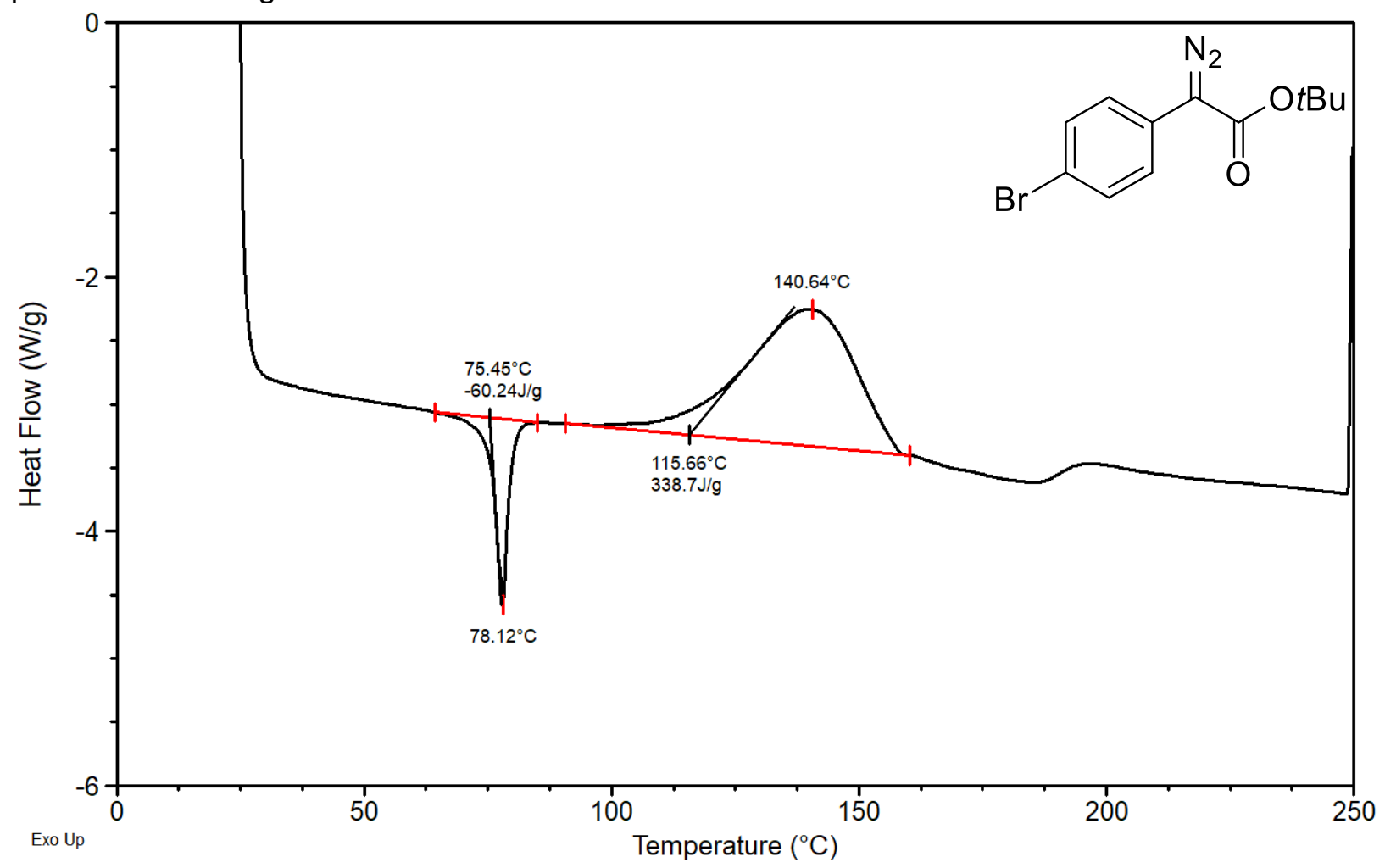

Figure S15. DSC Plot 2 for tert-butyl (4-bromophenyl)diazoacetate (35)

Sample mass $-3.6 \mathrm{mg}$

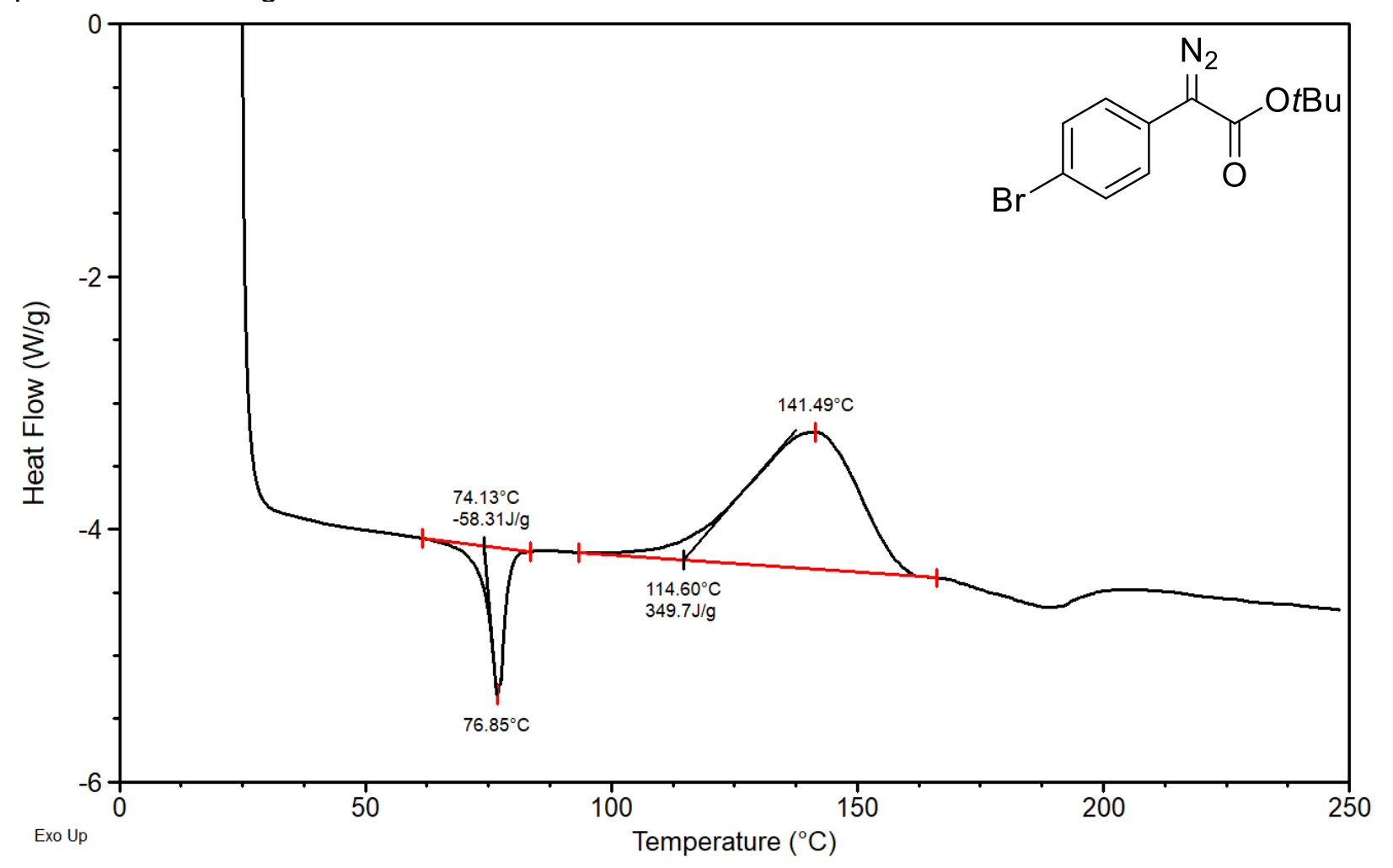


Figure S16. DSC Plot 1 for (1R,2S,5R)-2-isopropyl-5-methylcyclohexyl (phenyl)diazoacetate (36)

Sample mass $-3.5 \mathrm{mg}$

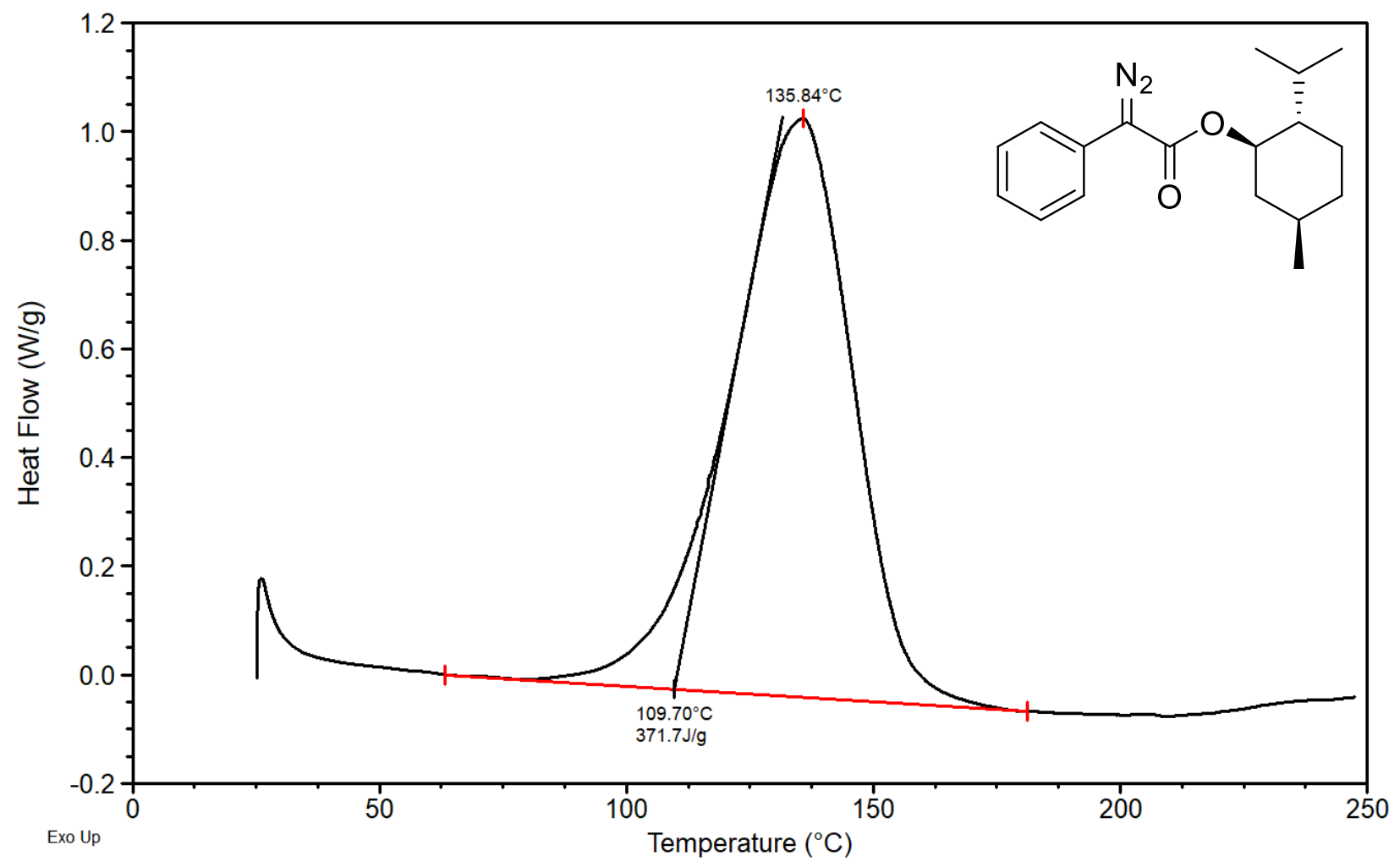

Figure S17. DSC Plot 2 for (1R,2S,5R)-2-isopropyl-5-methylcyclohexyl (phenyl)diazoacetate (36)

Sample mass $-5.3 \mathrm{mg}$

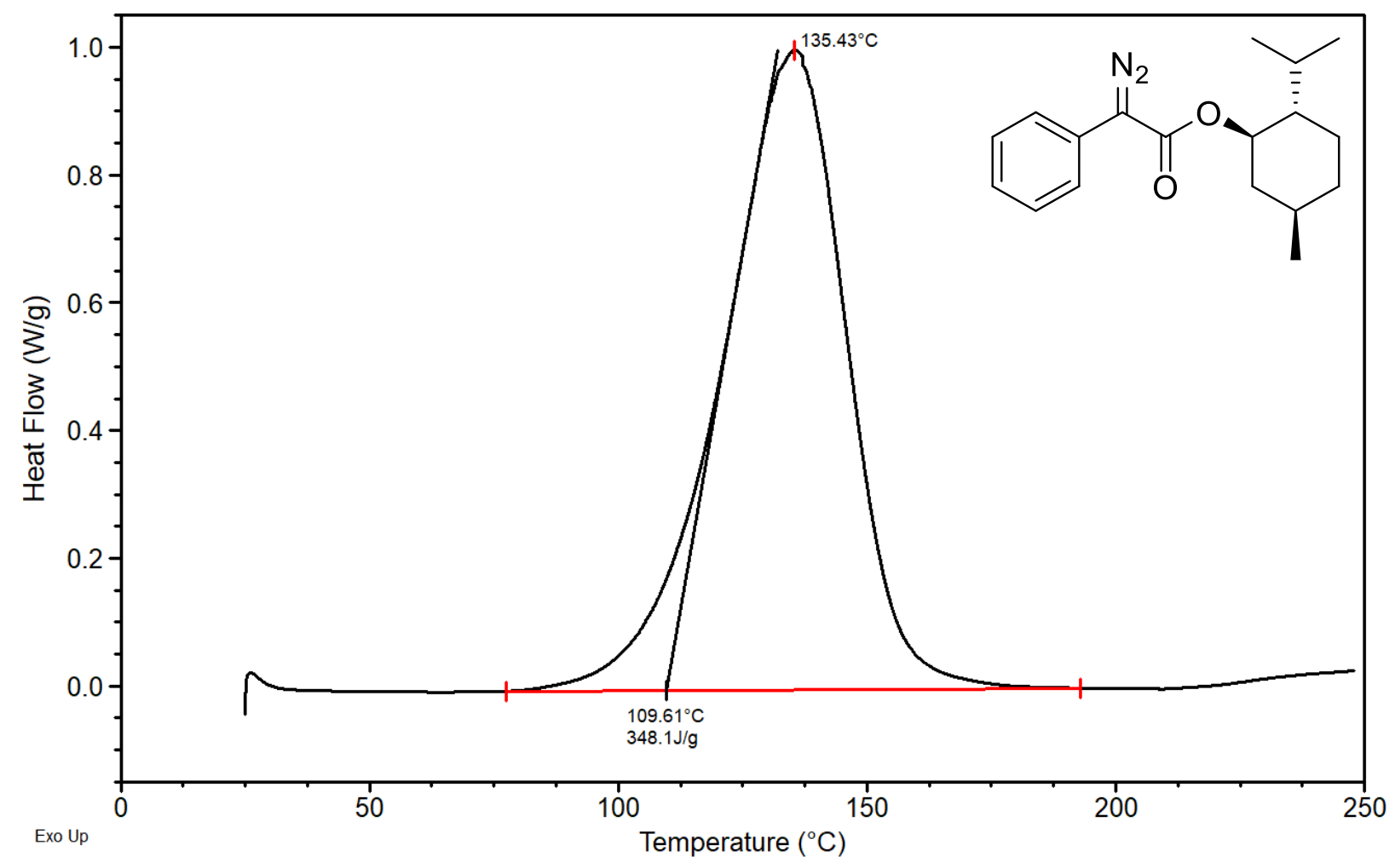


Figure S18. DSC Plot 12,2,2-trichloroethyl (phenyl)diazoacetate (37)

Sample mass $-5.5 \mathrm{mg}$

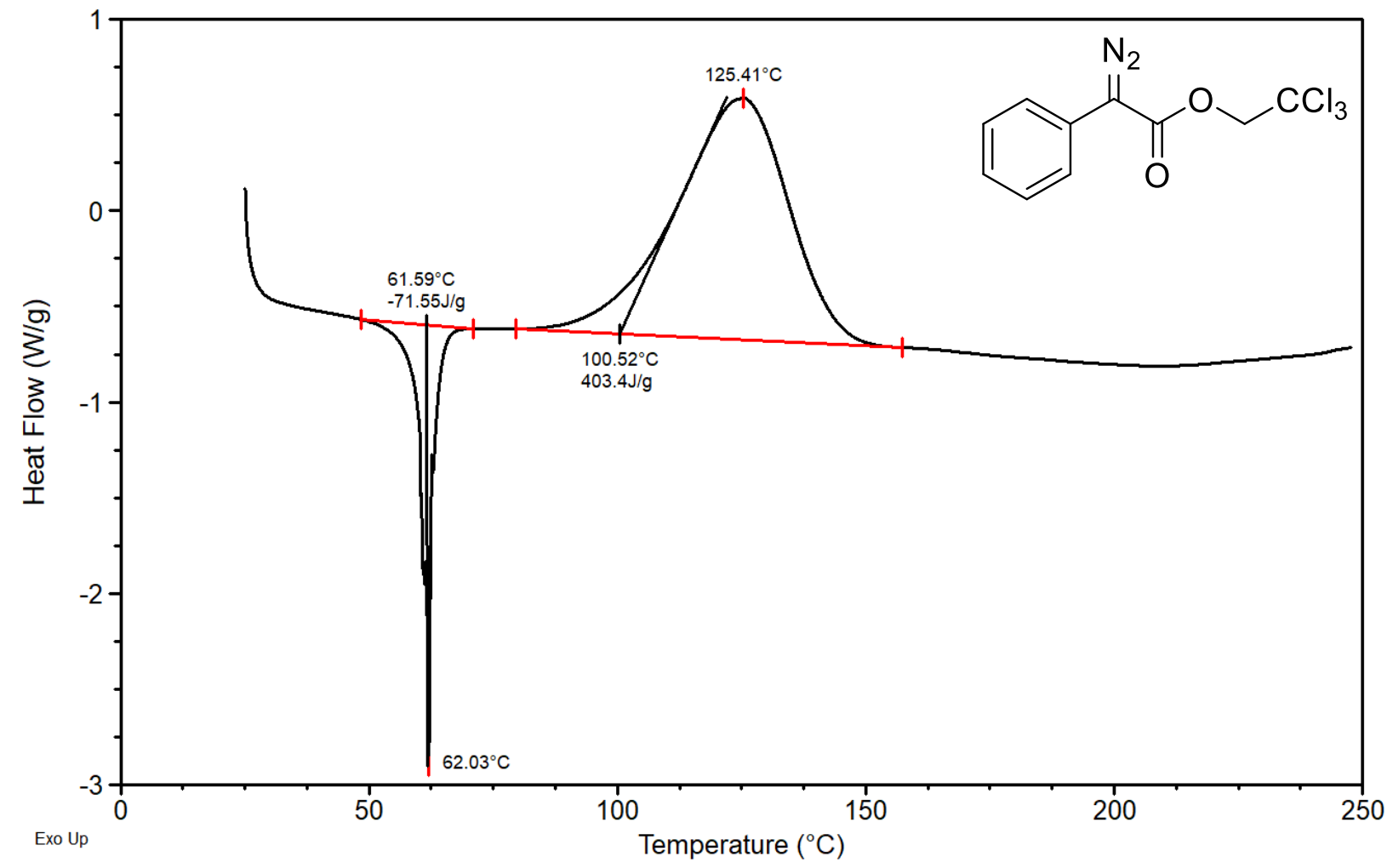

Figure S19. DSC Plot 2 for 2,2,2-trichloroethyl (phenyl)diazoacetate (37)

Sample mass $-5.2 \mathrm{mg}$

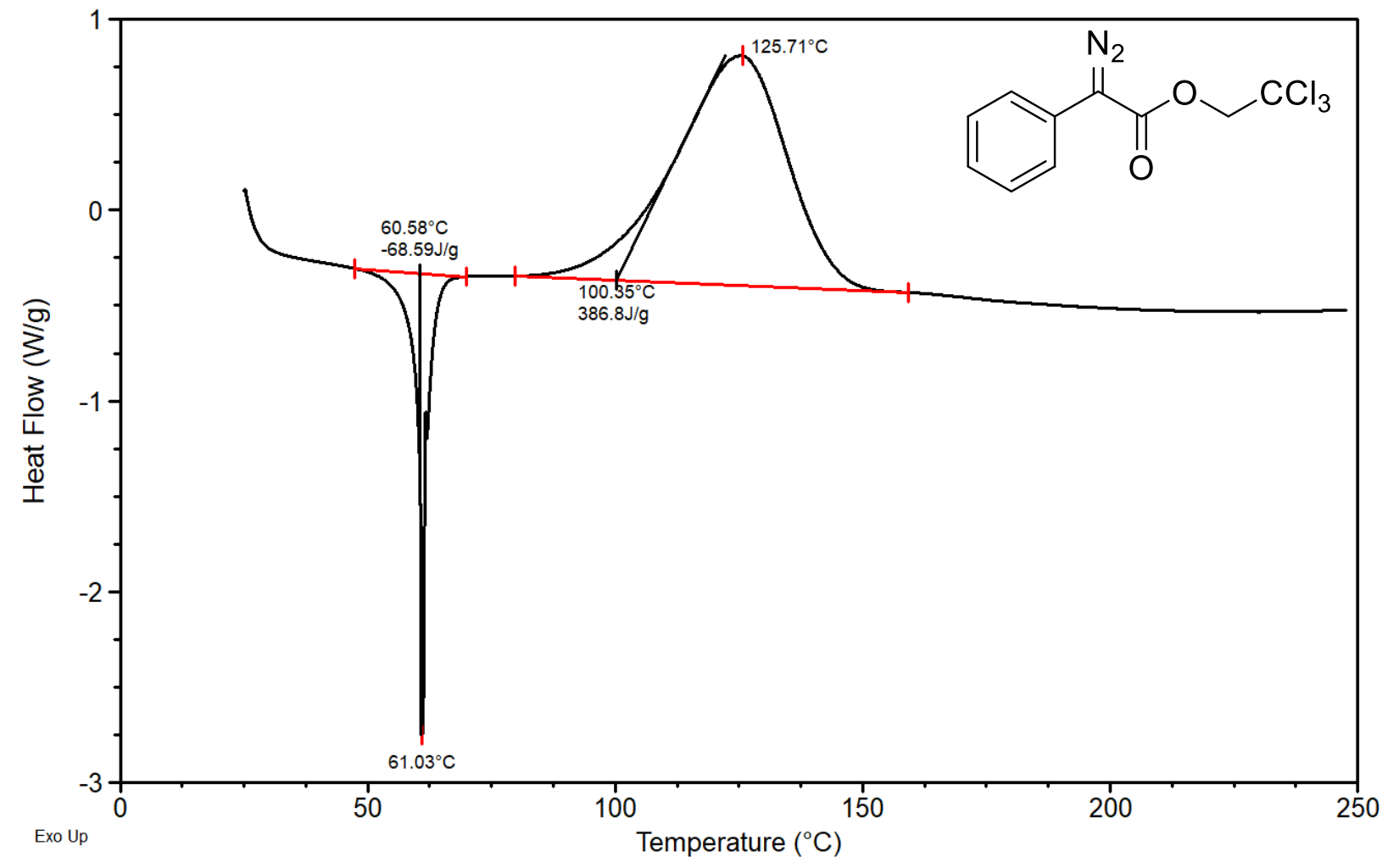


Figure S20. DSC Plot 1 for ethyl (4-methoxyphenyl)diazoacetate (38)

Sample mass $-3.2 \mathrm{mg}$

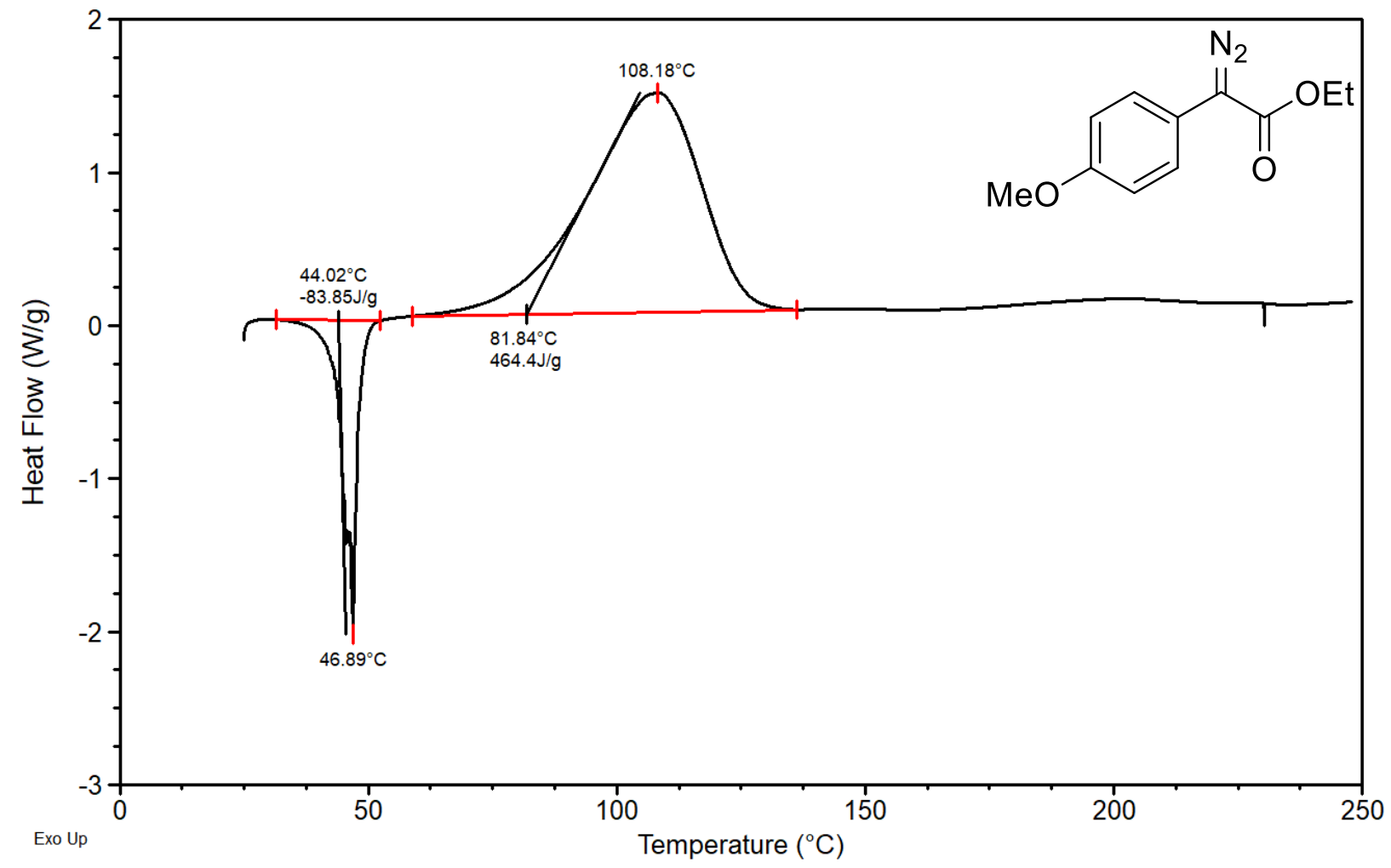

Figure S21. DSC Plot 2 for ethyl (4-methoxyphenyl)diazoacetate (38)

Sample mass $-4.7 \mathrm{mg}$

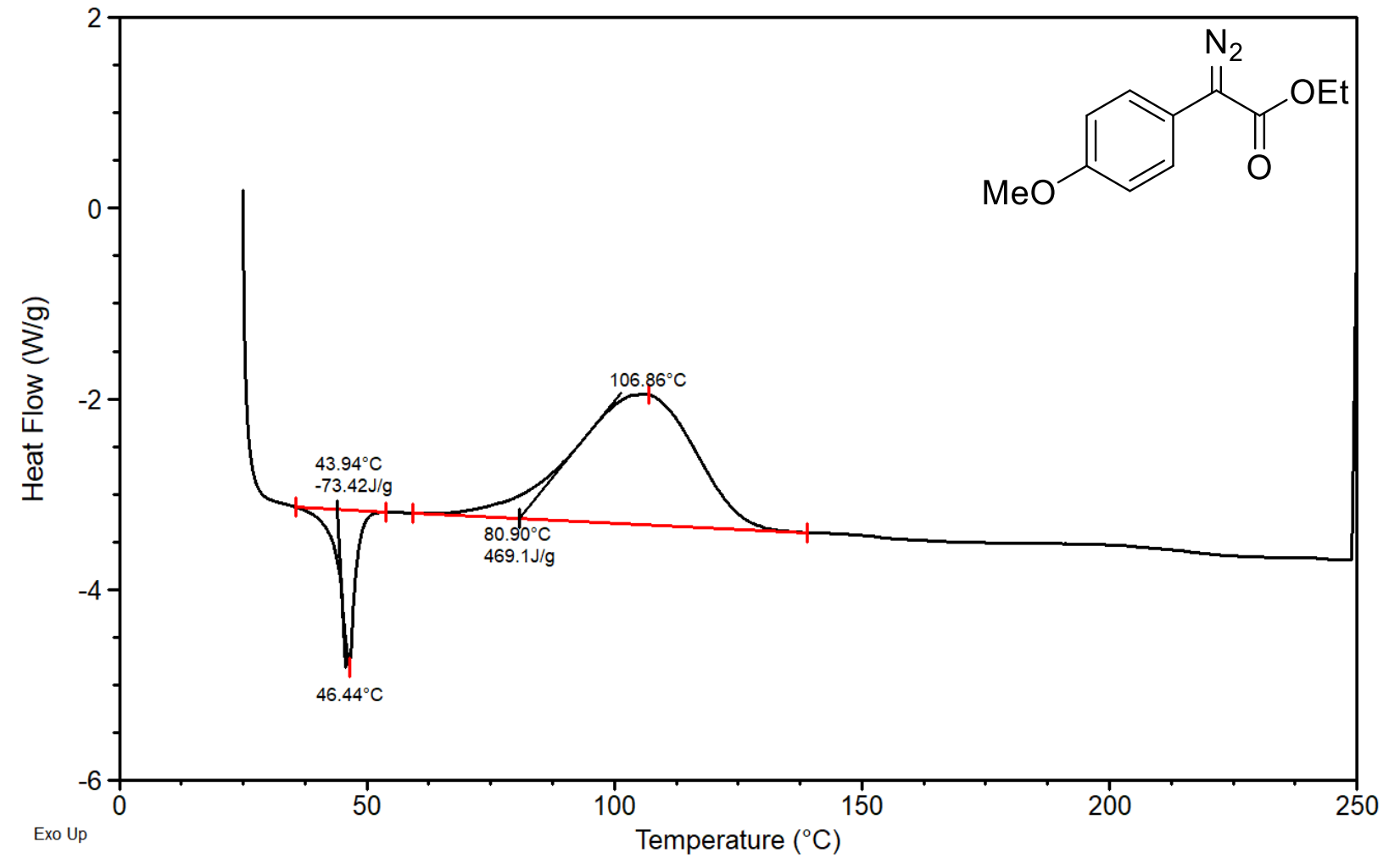


Figure S22. DSC Plot 1 for ethyl (4-methylphenyl)diazoacetate (39)

Sample mass $-5.3 \mathrm{mg}$

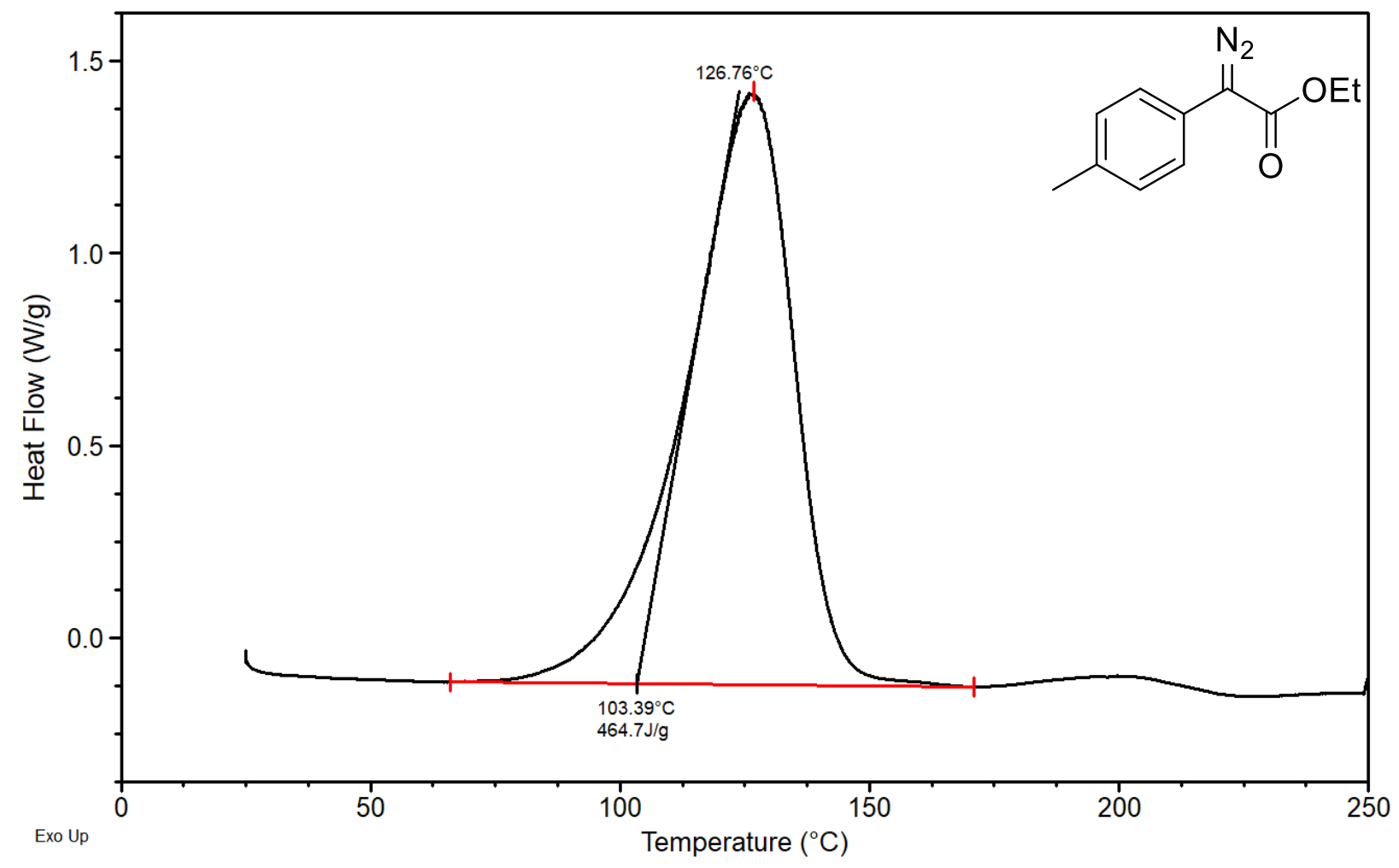

Figure S23. DSC Plot 2 for ethyl (4-methylphenyl)diazoacetate (39)

Sample mass $-3.9 \mathrm{mg}$

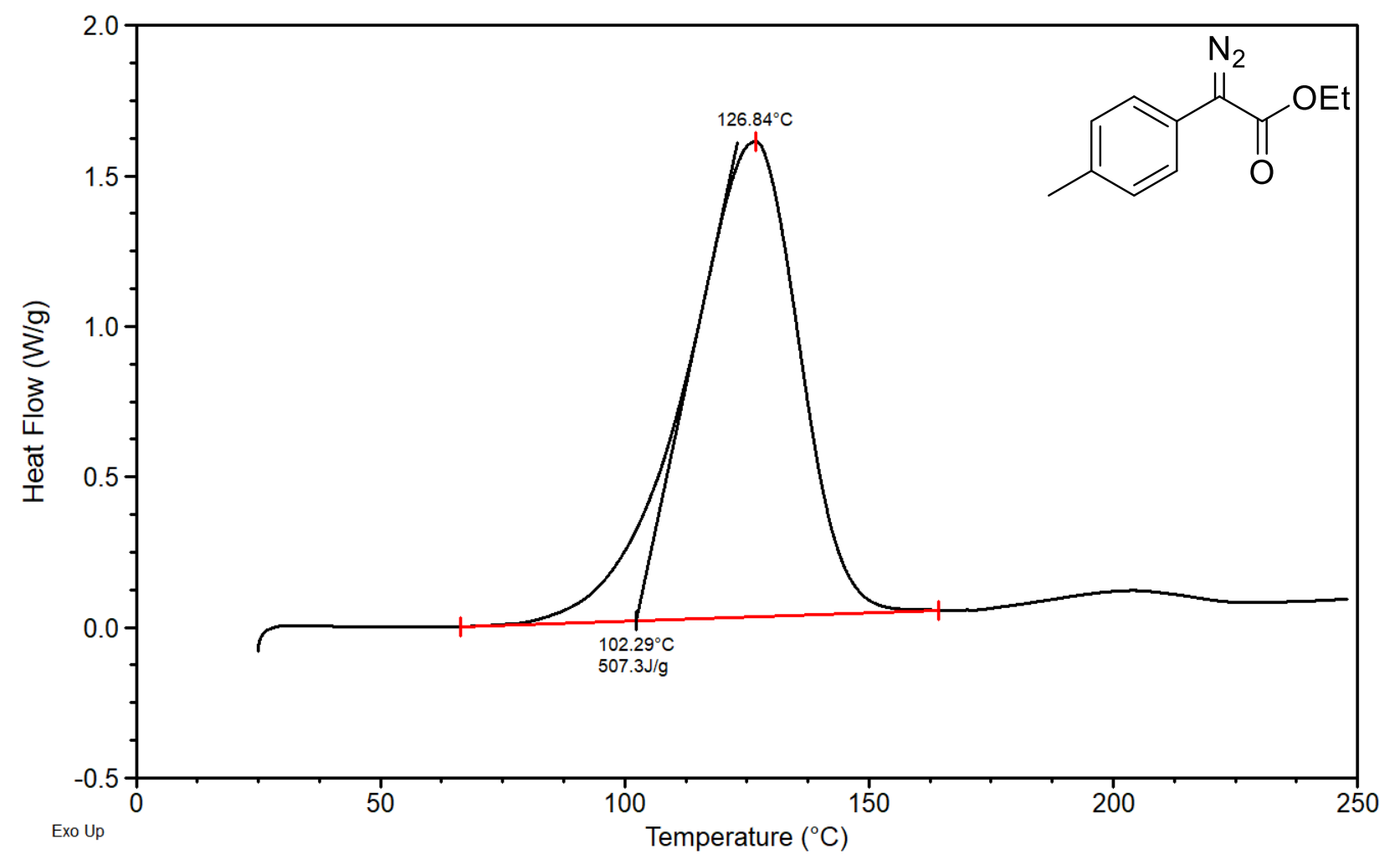


Figure S24. DSC Plot 1 for ethyl (4-fluorophenyl)diazoacetate (40)

Sample mass $-6.4 \mathrm{mg}$

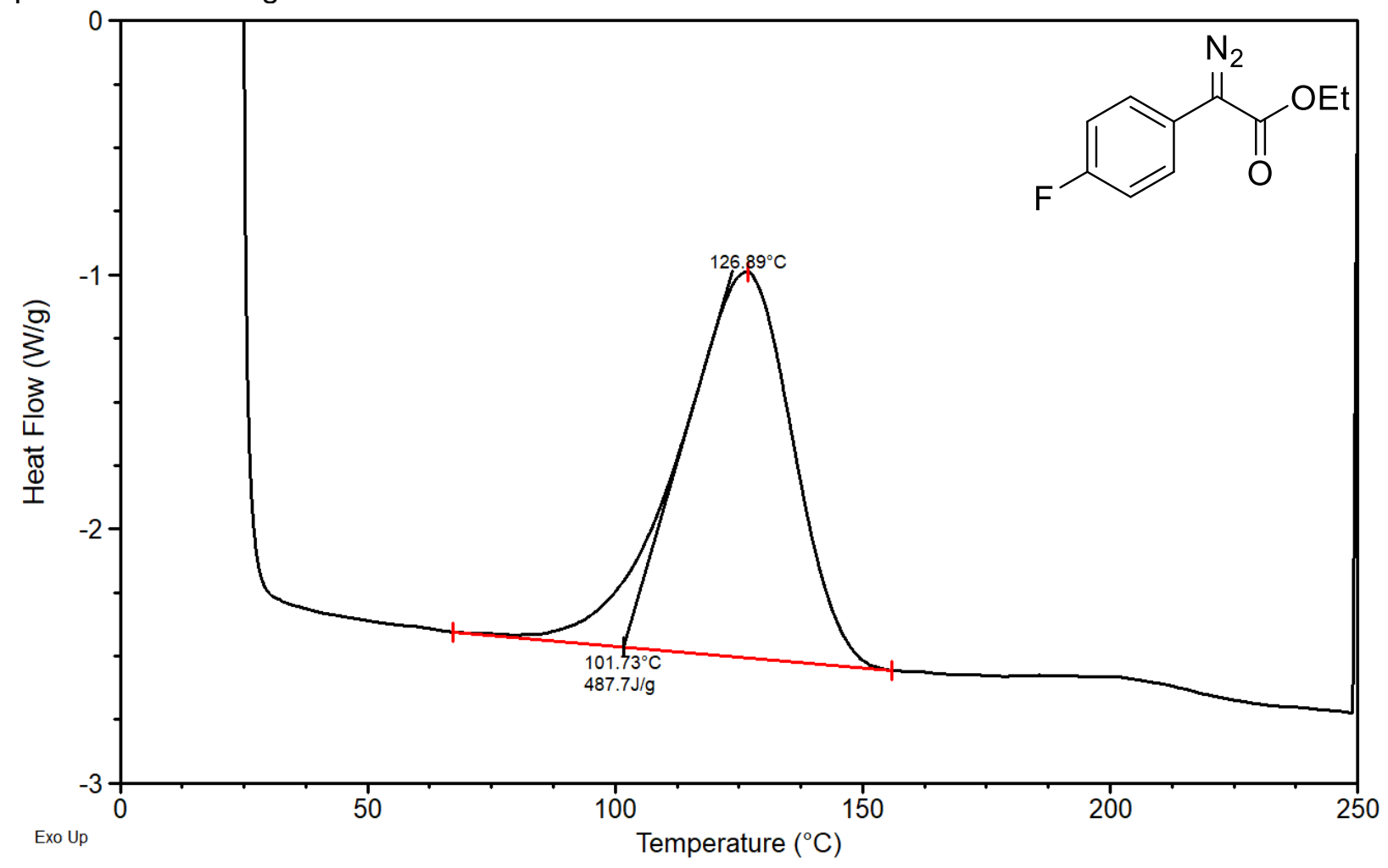

Figure S25. DSC Plot 2 for ethyl (4-fluorophenyl)diazoacetate (40)

Sample mass $-3.4 \mathrm{mg}$

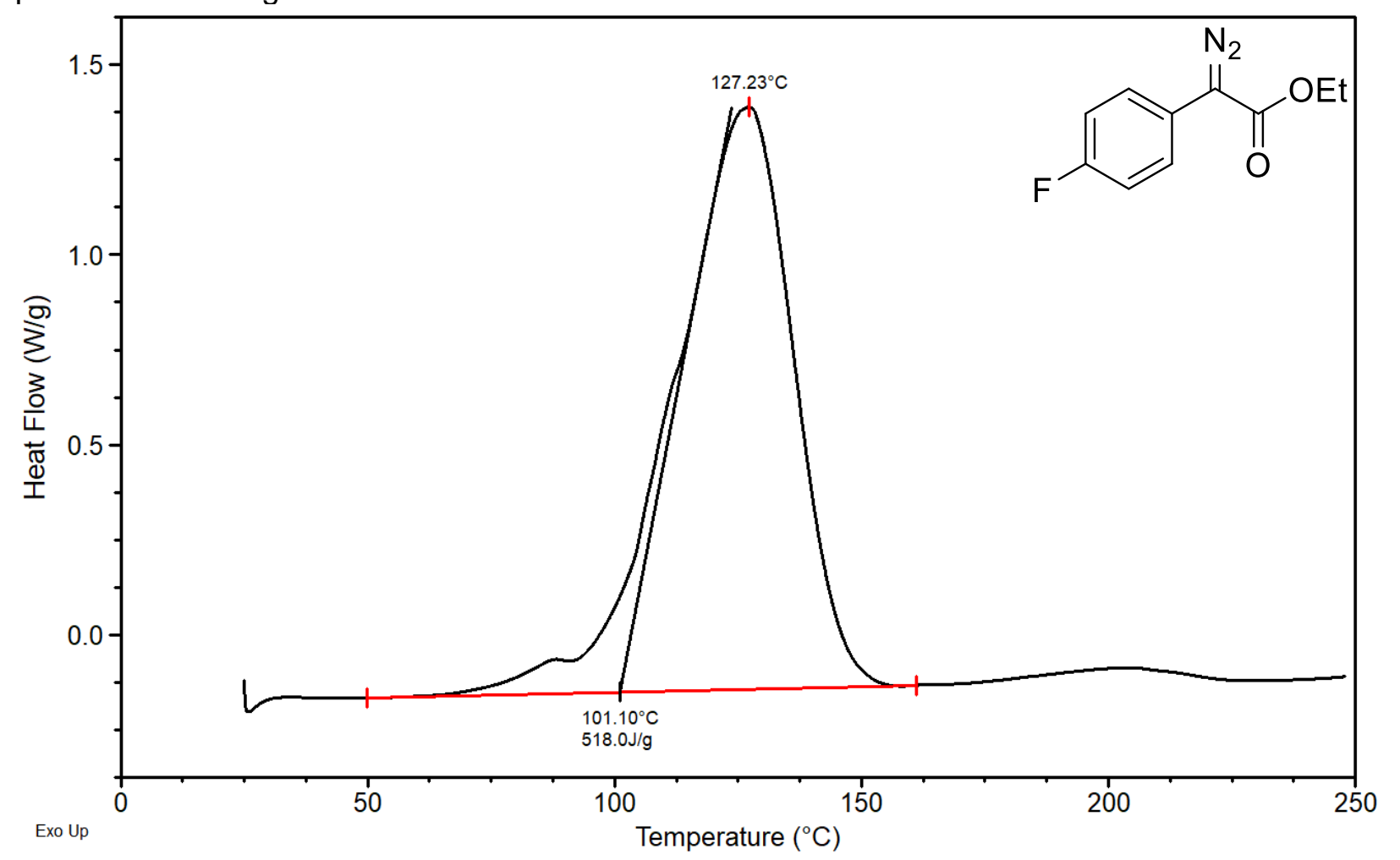


Figure S26. DSC Plot 1 for ethyl (4-chlorophenyl)diazoacetate (41)

Sample mass $-4.6 \mathrm{mg}$

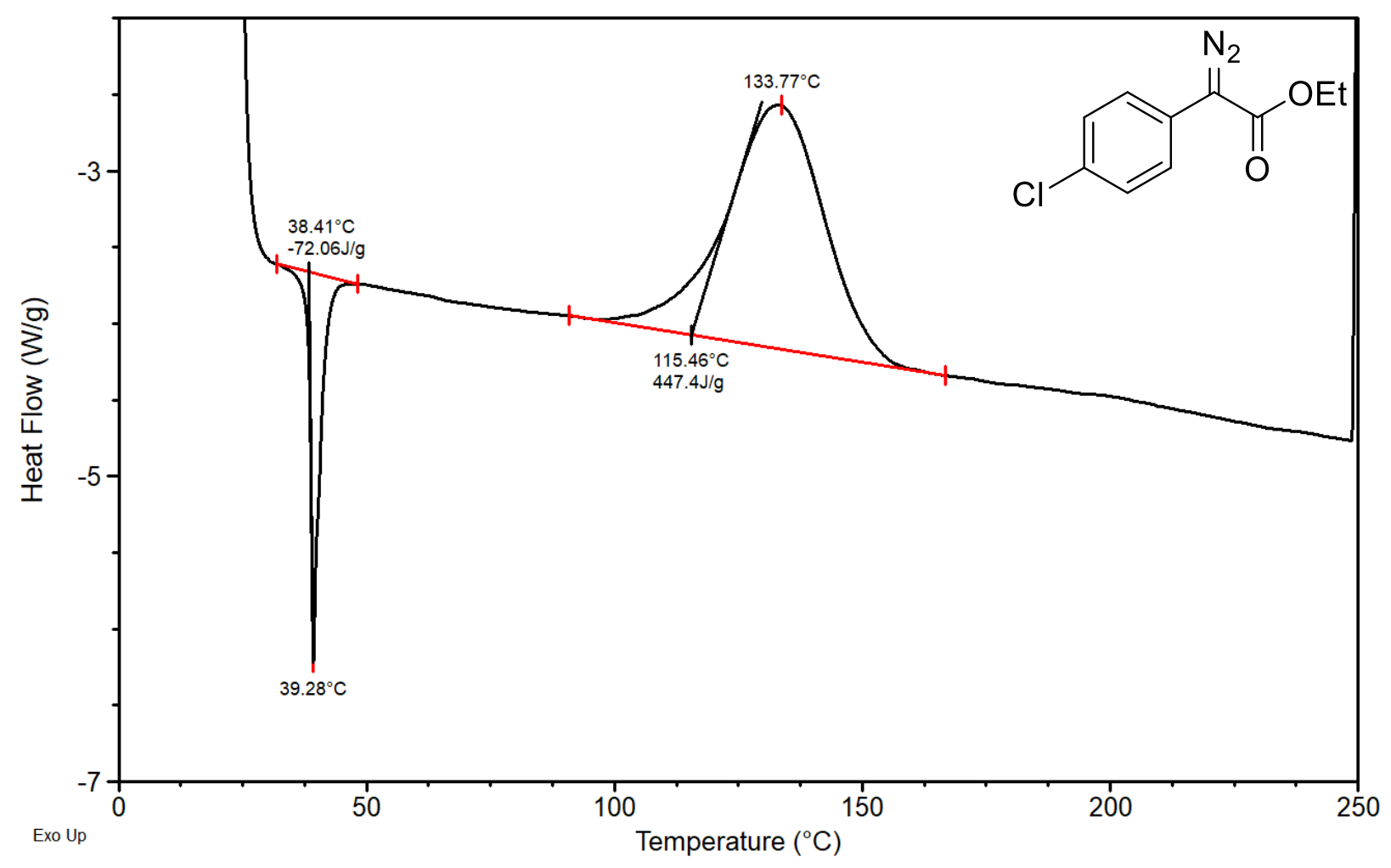

Figure S27. DSC Plot 2 for ethyl (4-chlorophenyl)diazoacetate (41)

Sample mass $-2.0 \mathrm{mg}$

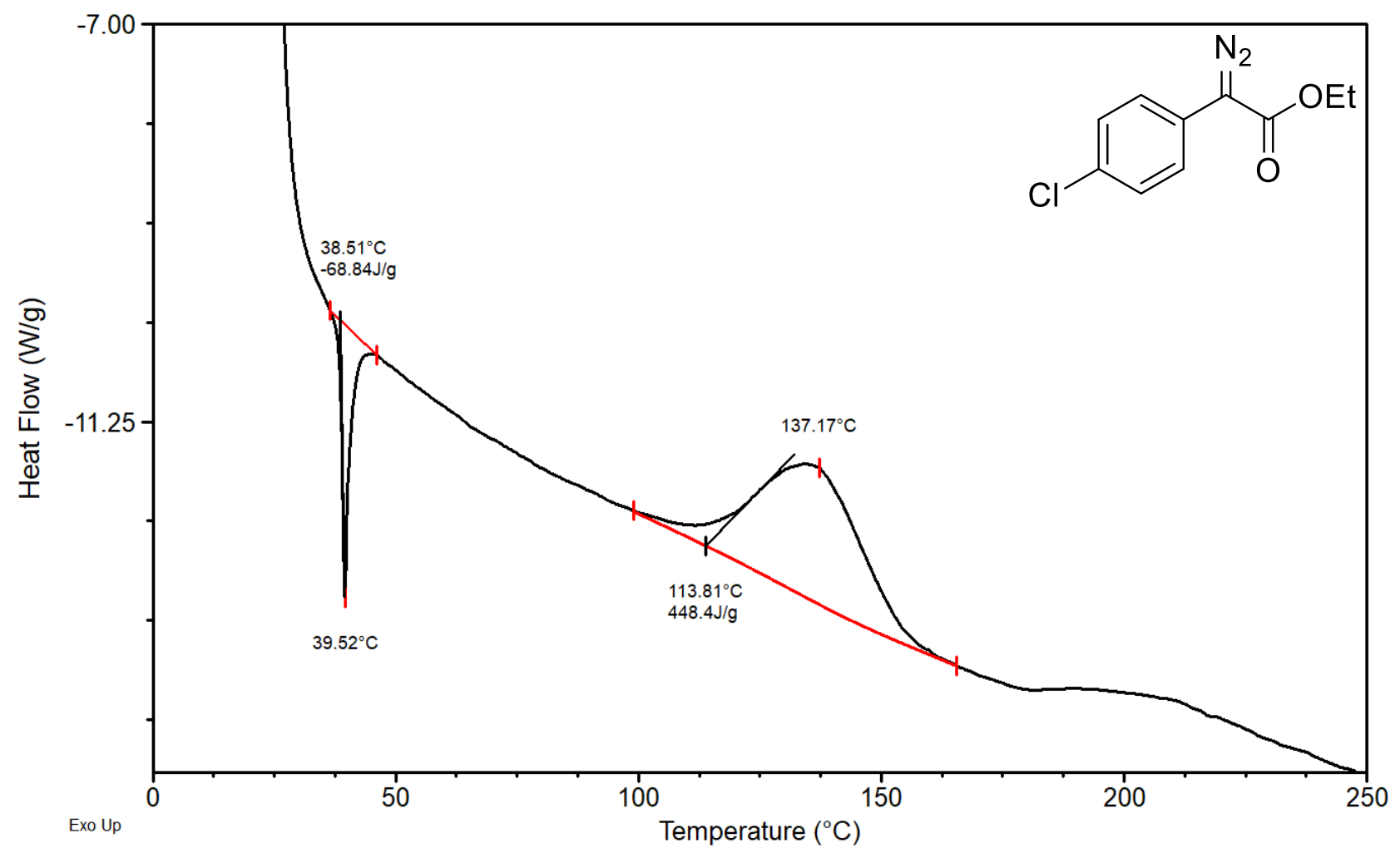


Figure S28. DSC Plot 1 for ethyl (4-bromophenyl)diazoacetate (42)

Sample mass $-5.4 \mathrm{mg}$

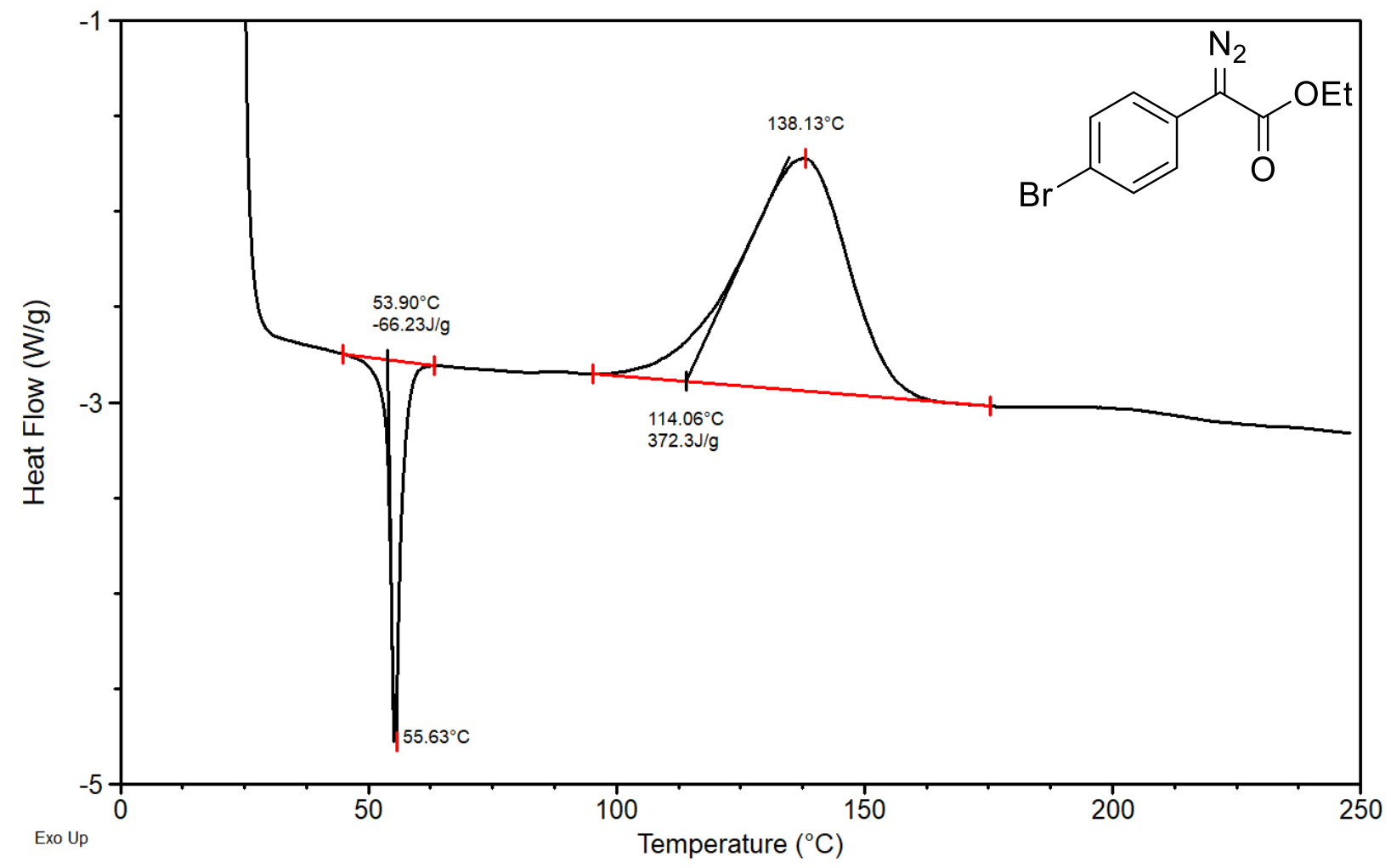

Figure S29. DSC Plot 2 for ethyl (4-bromophenyl)diazoacetate (42)

Sample mass $-3.1 \mathrm{mg}$

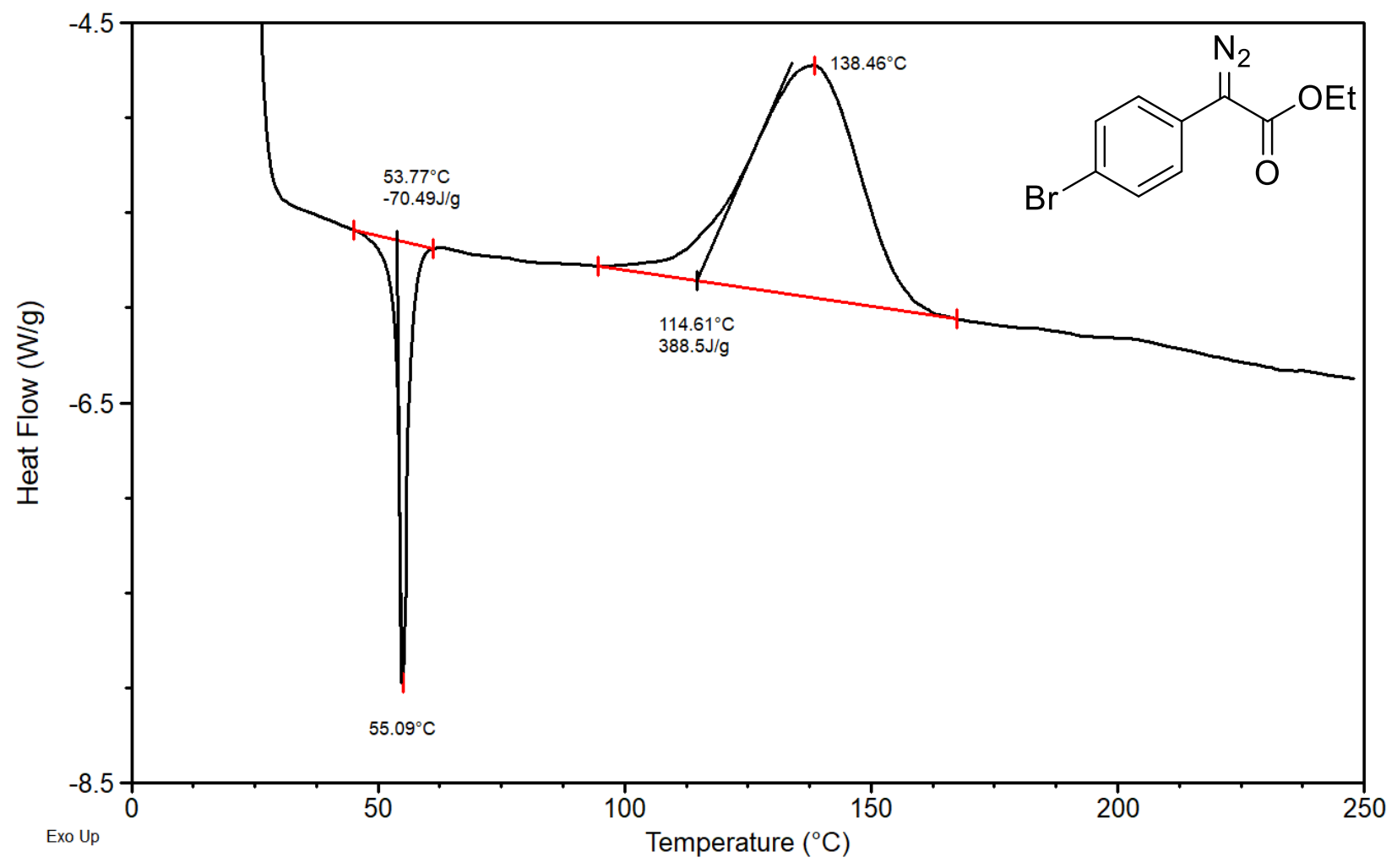


Figure S30. DSC Plot 1 for ethyl (4-trifluoromethylphenyl)diazoacetate (43)

Sample mass $-3.0 \mathrm{mg}$

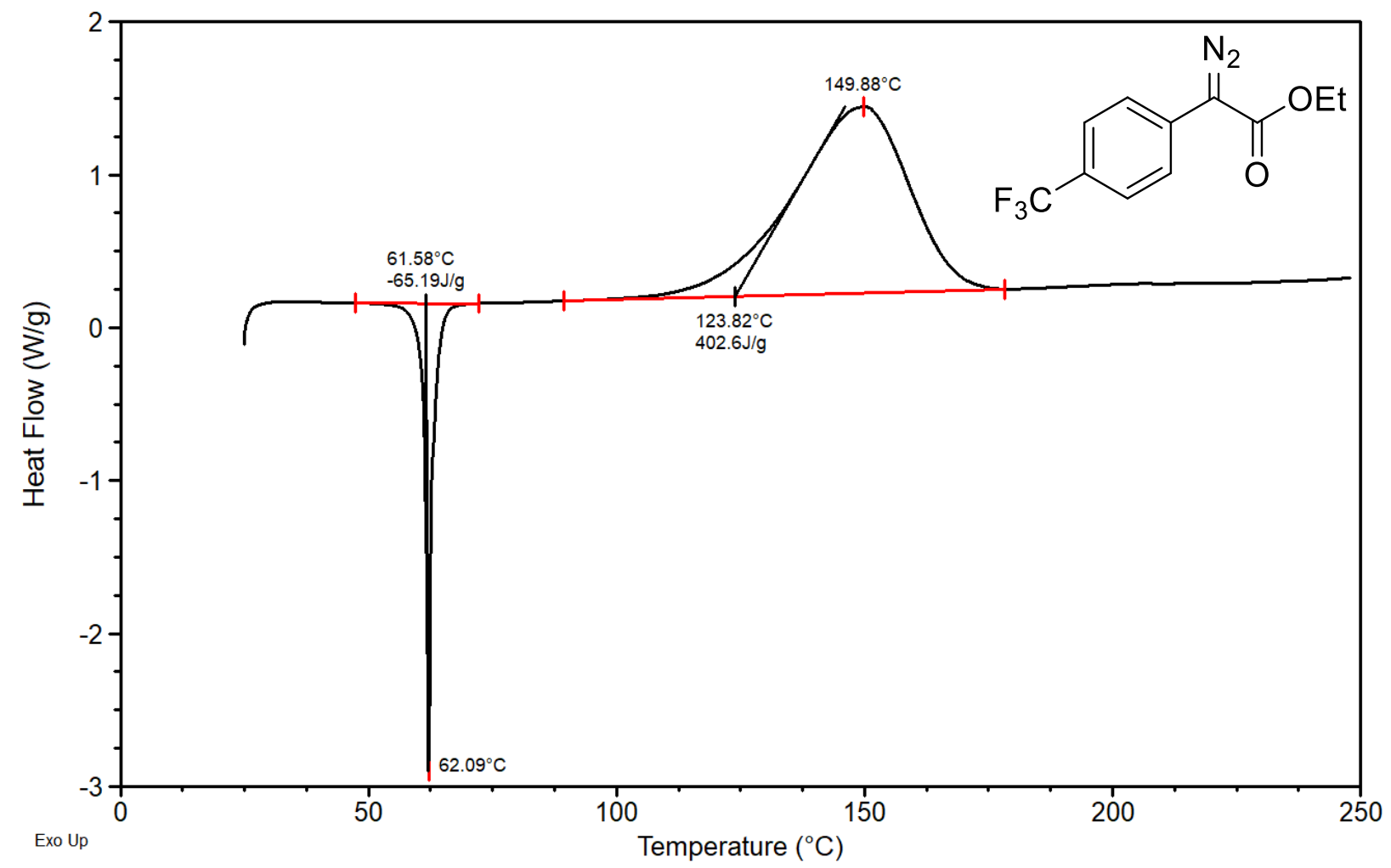

Figure S31. DSC Plot 2 for ethyl (4-trifluoromethylphenyl)diazoacetate (43)

Sample mass $-1.4 \mathrm{mg}$

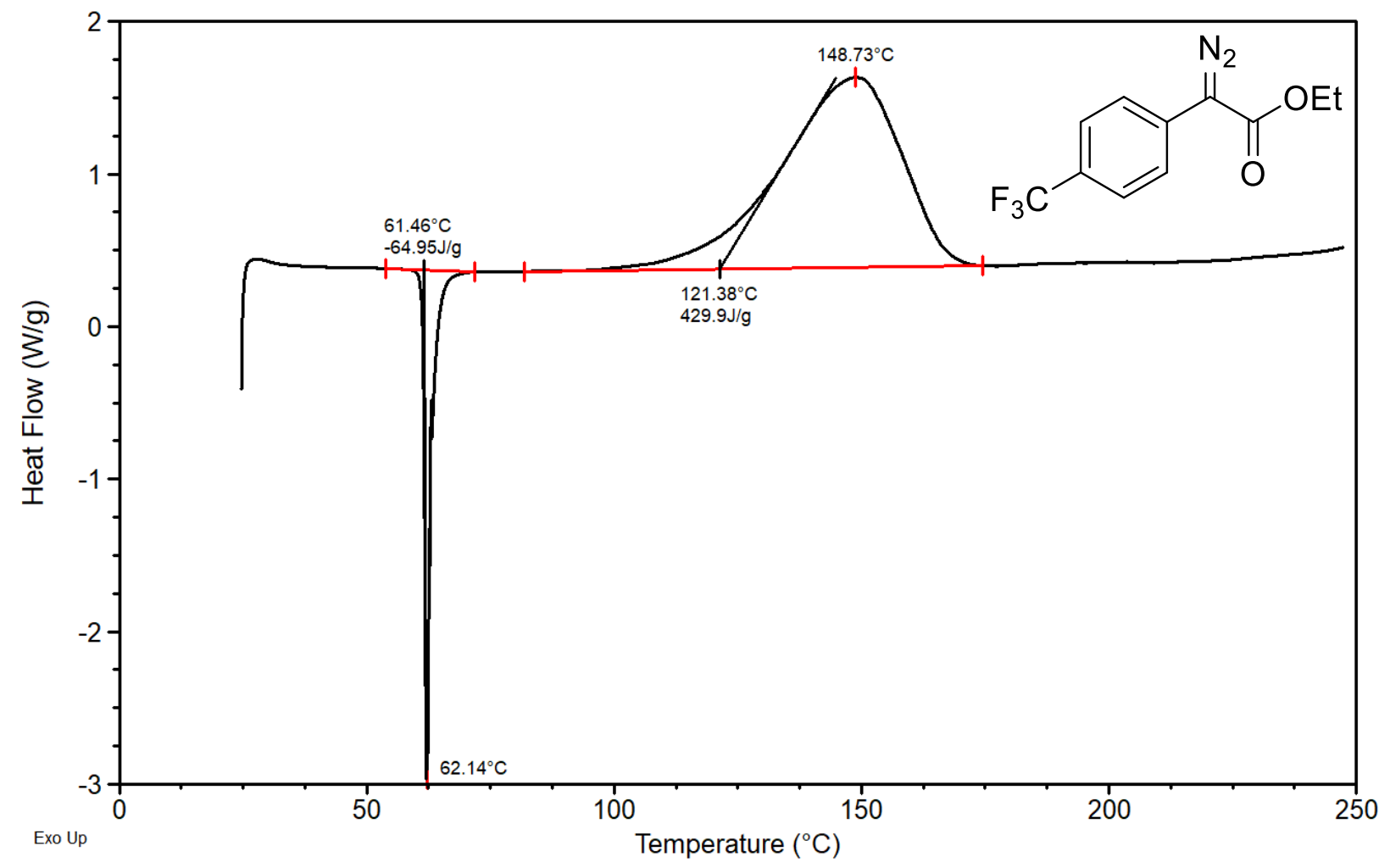


Figure S32. DSC Plot 1 for ethyl (4-nitrophenyl)diazoacetate (44)

Sample mass $-2.1 \mathrm{mg}$

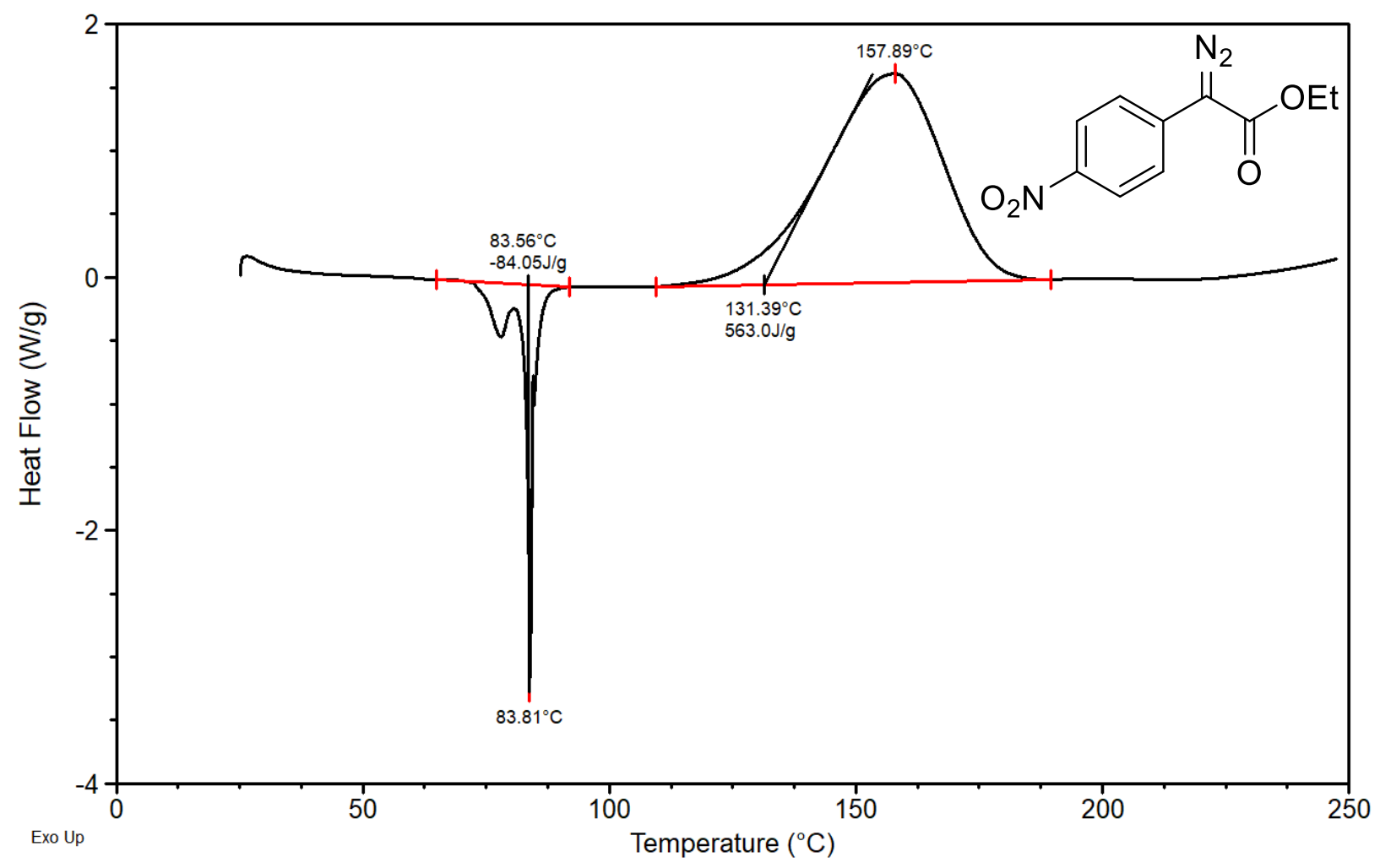

Figure S33. DSC Plot 2 for ethyl (4-nitrophenyl)diazoacetate (44)

Sample mass $-1.5 \mathrm{mg}$

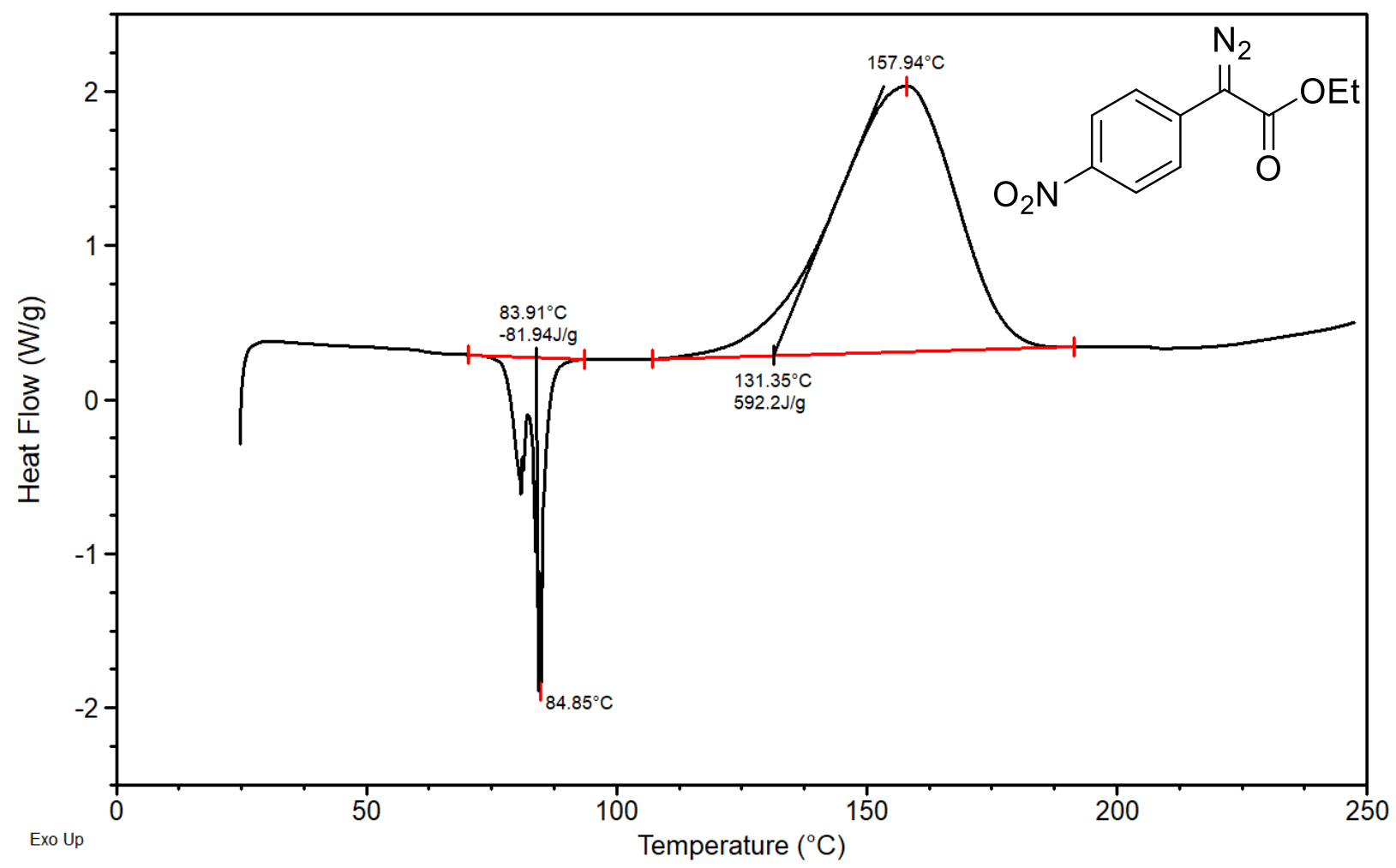


Figure S34. DSC Plot 1 for ethyl (3-methoxyphenyl)diazoacetate (45)

Sample mass $-3.2 \mathrm{mg}$

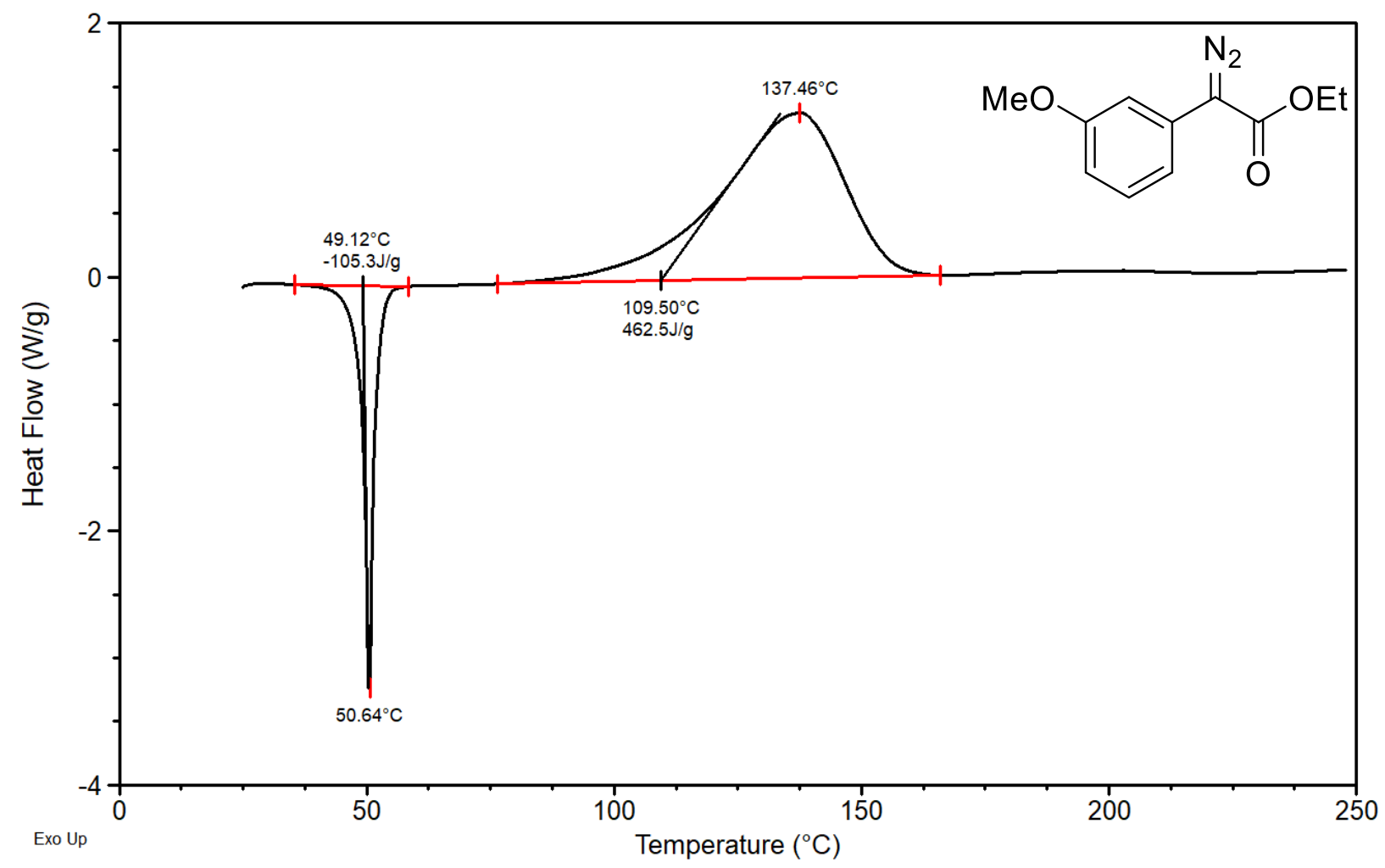

Figure S35. DSC Plot 2 for ethyl (3-methoxyphenyl)diazoacetate (45)

Sample mass $-4.2 \mathrm{mg}$

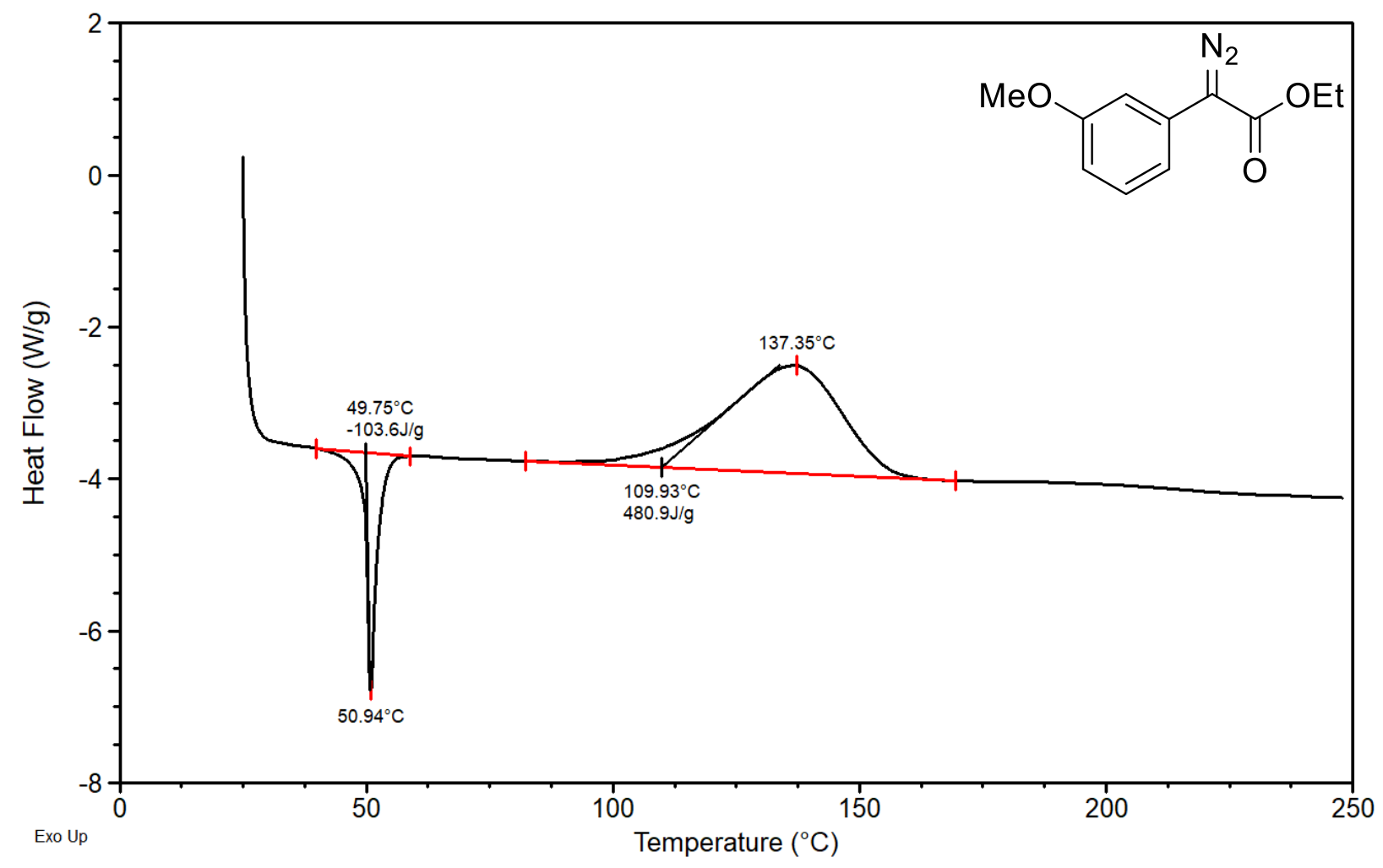


Figure S36. DSC Plot 1 for ethyl (3-methylphenyl)diazoacetate (46)

Sample mass $-5.5 \mathrm{mg}$

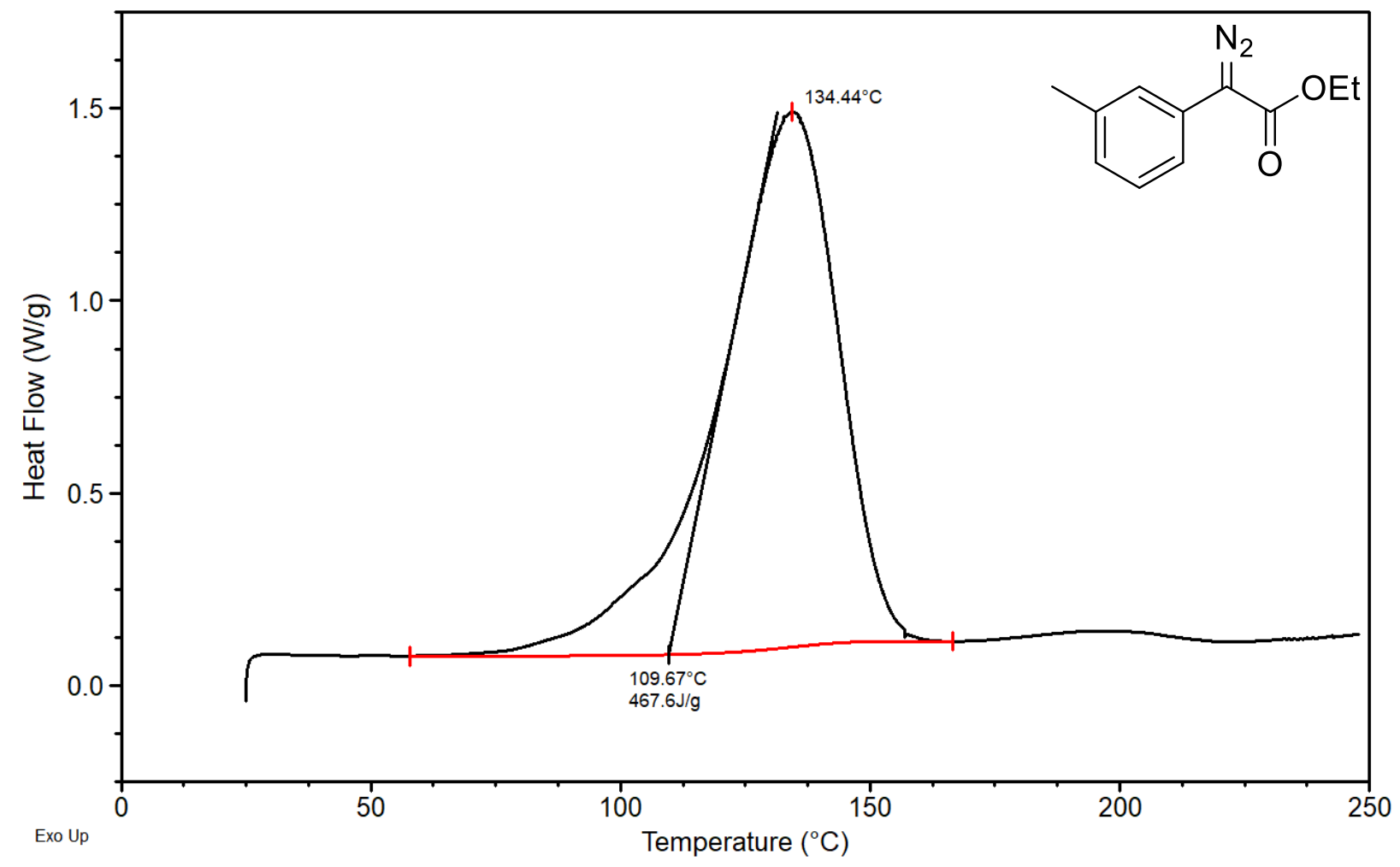

Figure S37. DSC Plot 2 for ethyl (3-methylphenyl)diazoacetate (46)

Sample mass $-3.4 \mathrm{mg}$

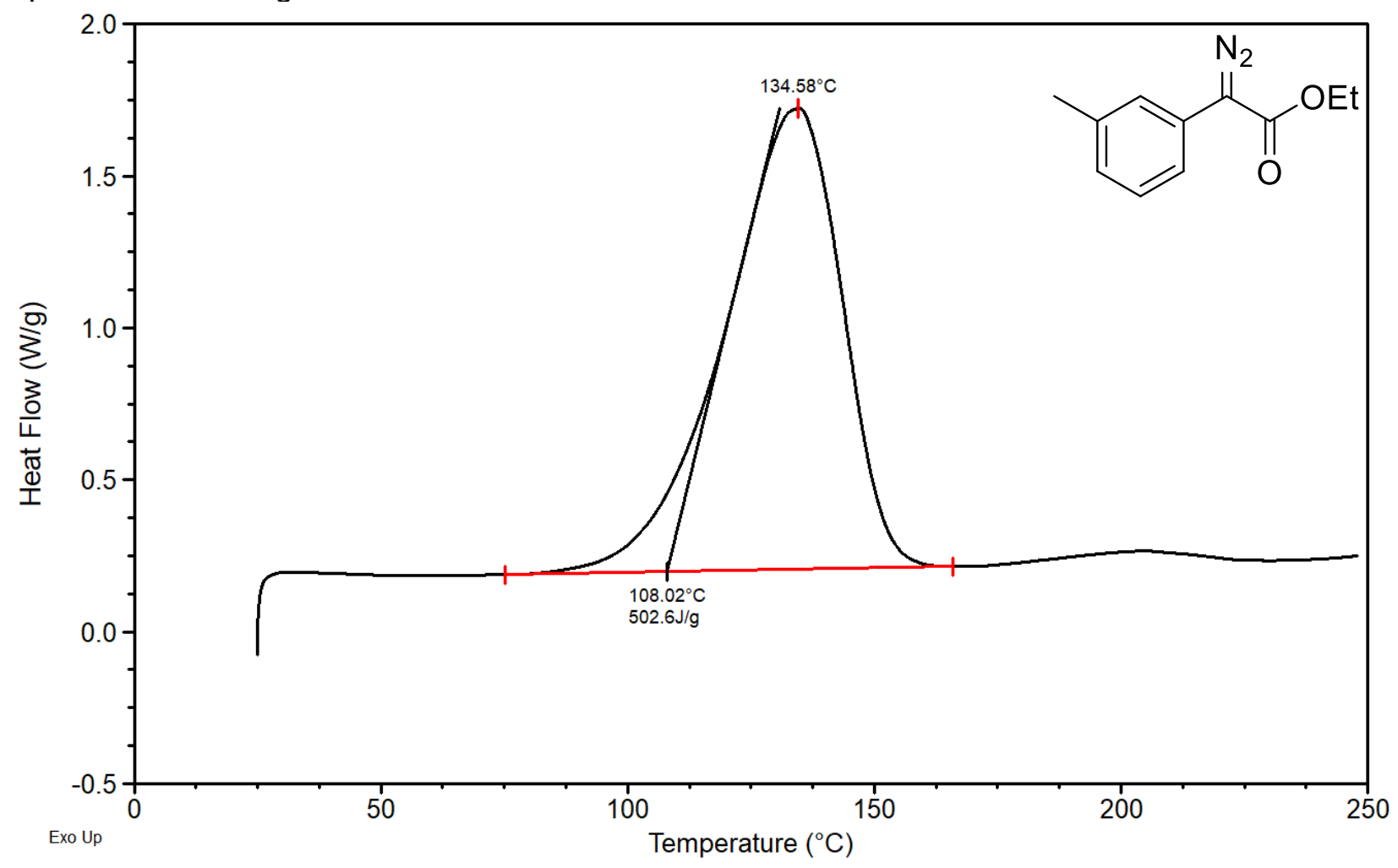


Figure S38. DSC Plot 1 for ethyl (3-fluorophenyl)diazoacetate (47)

Sample mass $-5.1 \mathrm{mg}$

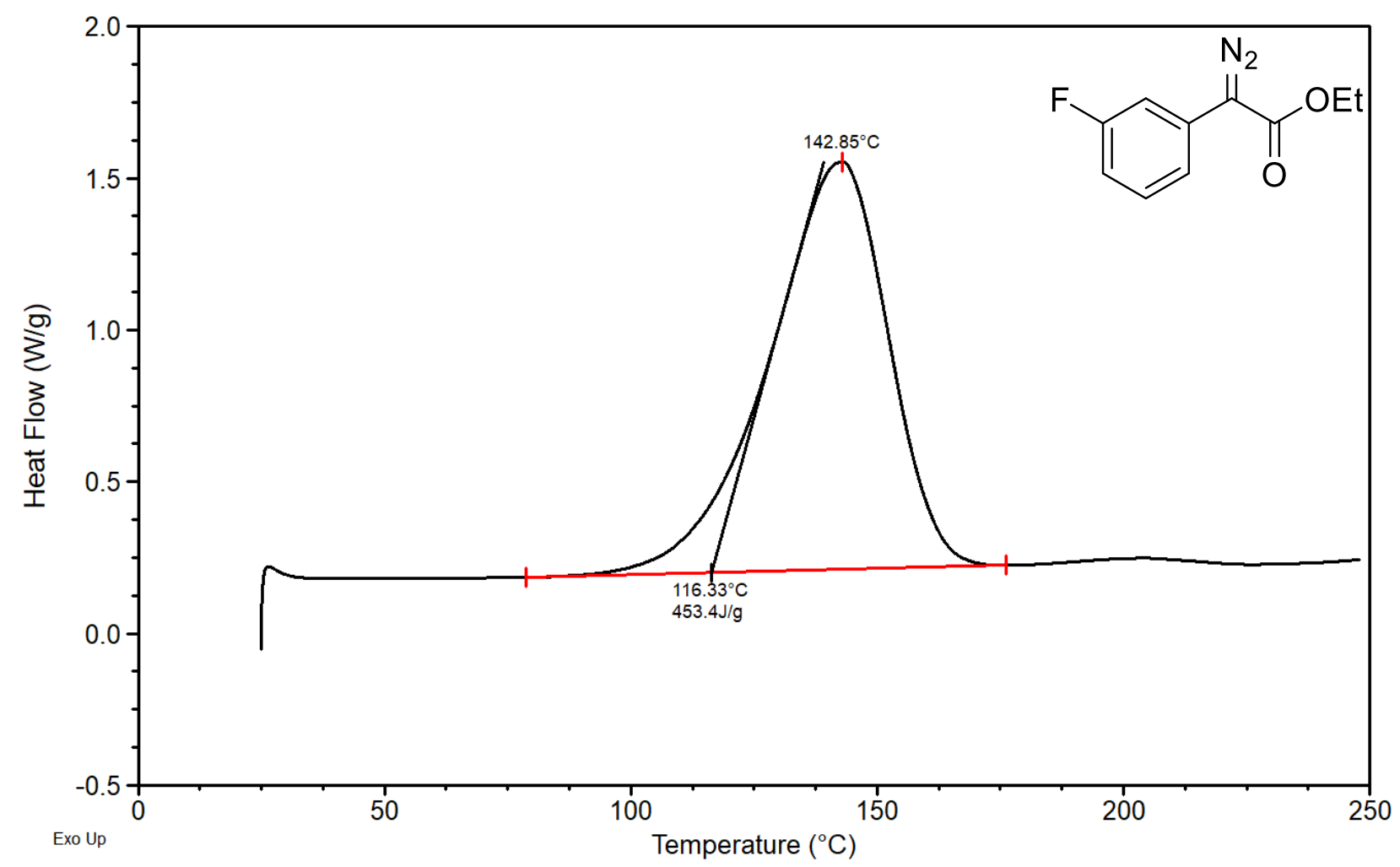

Figure S39. DSC Plot 2 for ethyl (3-fluorophenyl)diazoacetate (47)

Sample mass $-5.1 \mathrm{mg}$

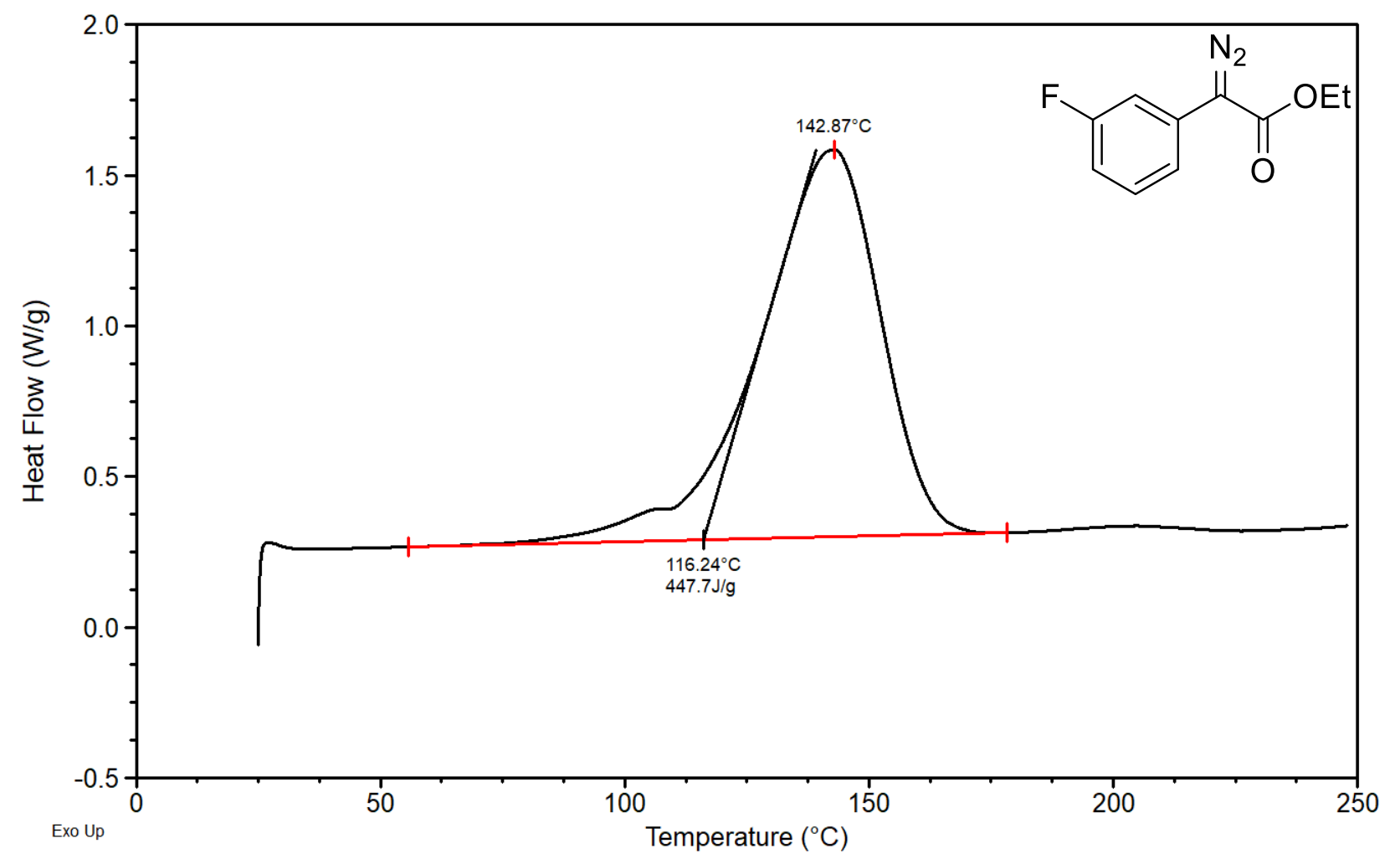


Figure S40. DSC Plot 1 for ethyl (3-chlorophenyl)diazoacetate (48)

Sample mass $-4.1 \mathrm{mg}$

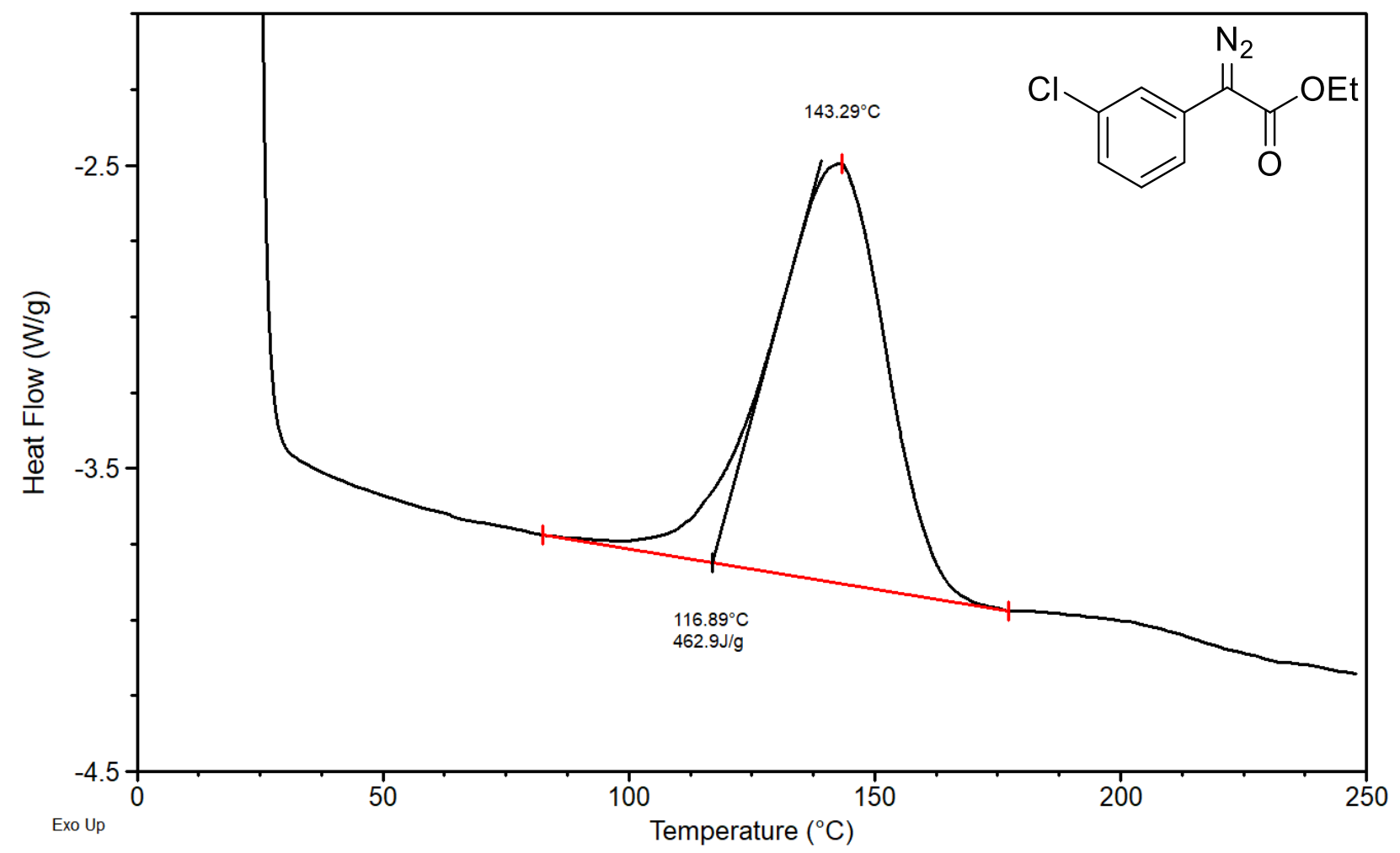

Figure S41. DSC Plot 2 for ethyl (3-chlorophenyl)diazoacetate (48)

Sample mass $-6.2 \mathrm{mg}$

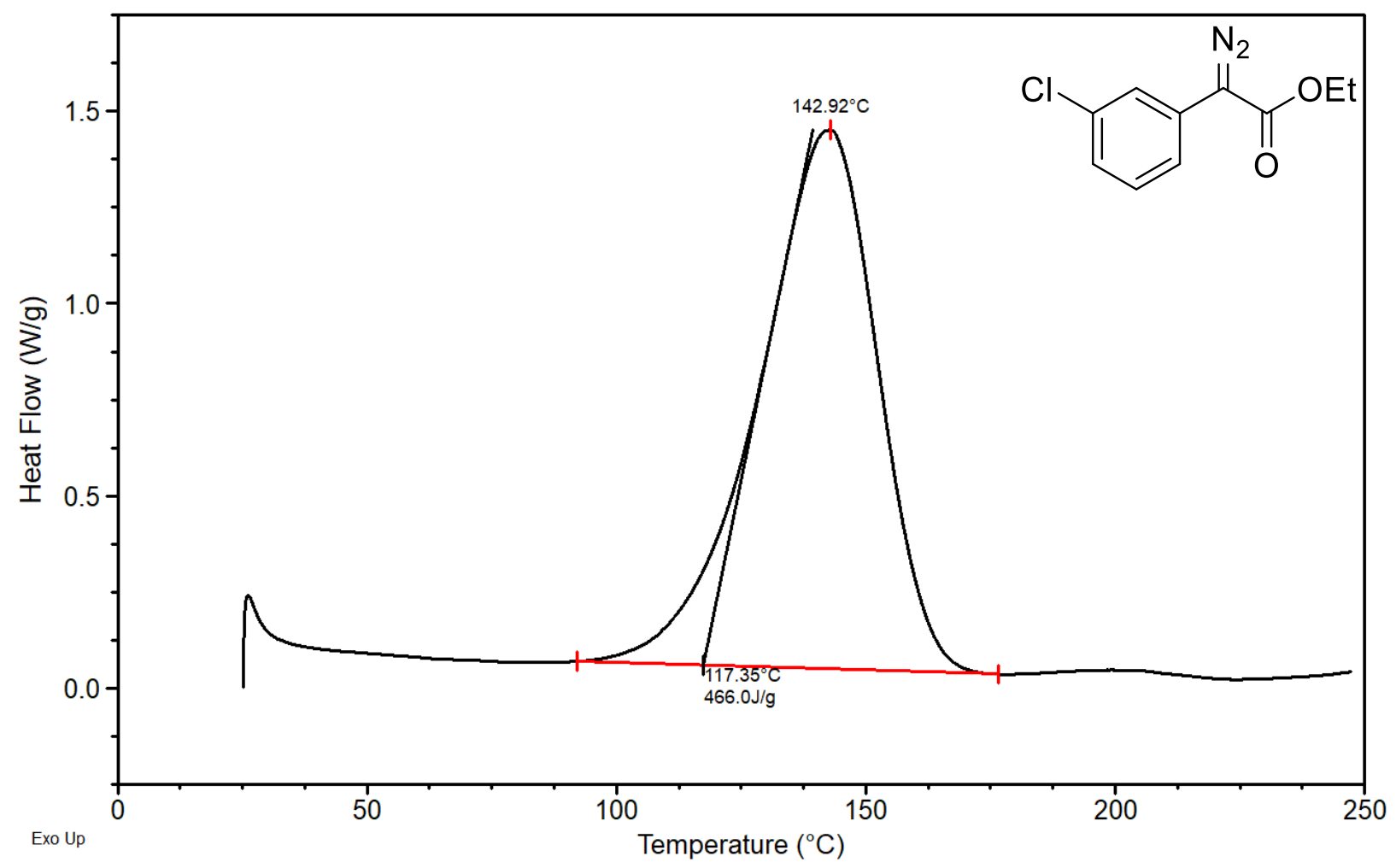


Figure S42. DSC Plot 1 for ethyl (3-trifluoromethylphenyl)diazoacetate (49)

Sample mass $-5.2 \mathrm{mg}$

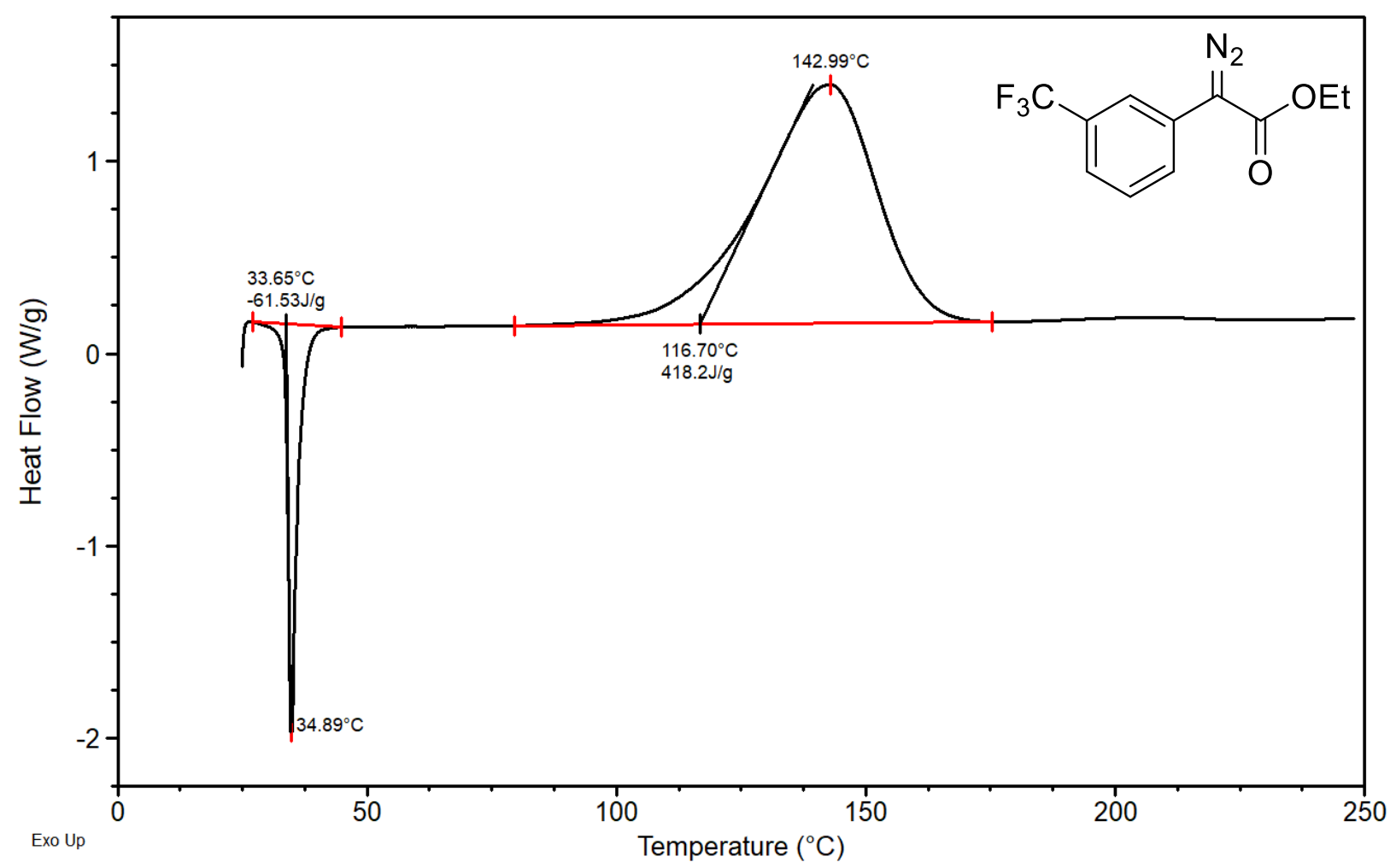

Figure S43. DSC Plot 2 for ethyl (3-trifluoromethylphenyl)diazoacetate (49)

Sample mass $-5.3 \mathrm{mg}$

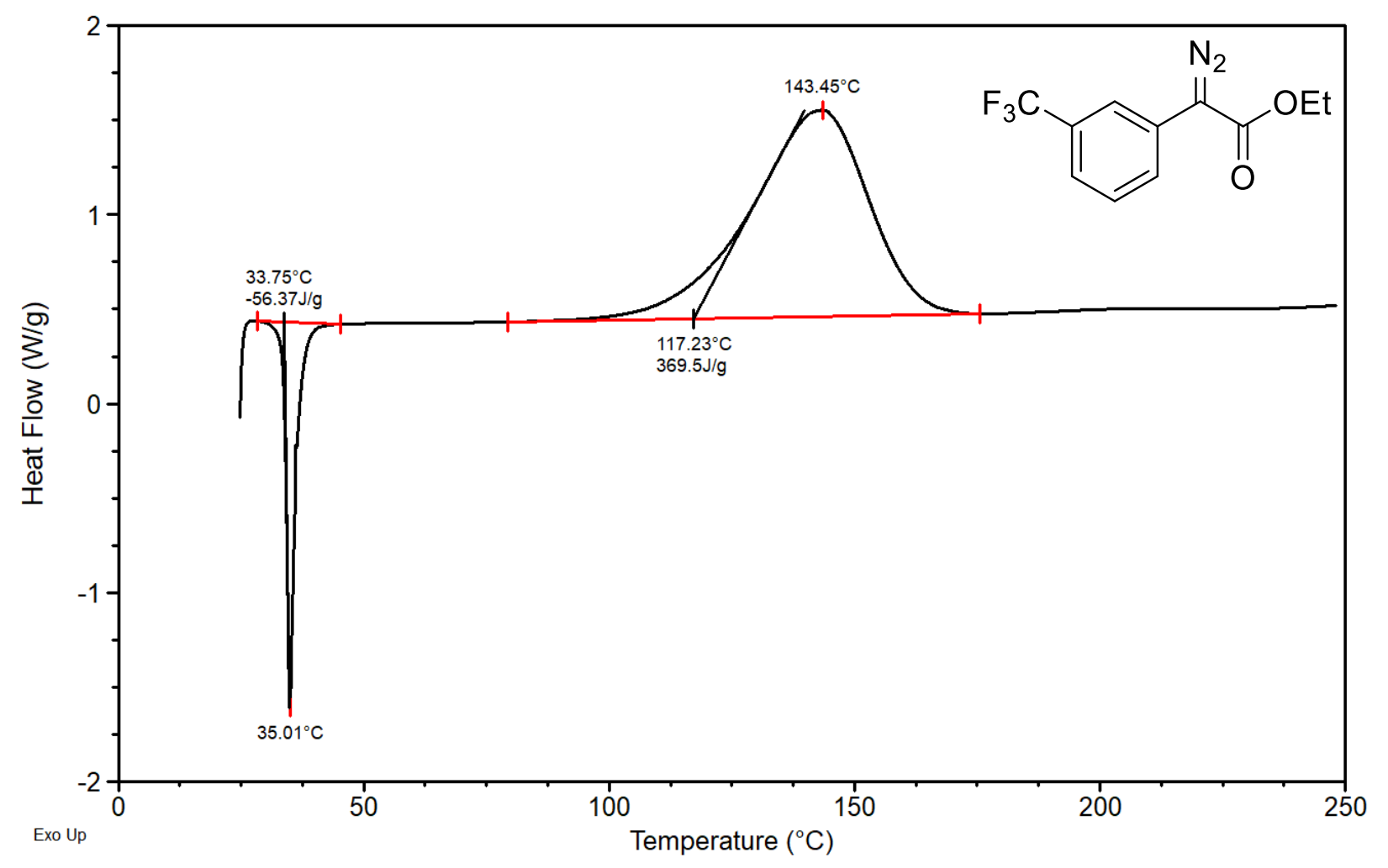


Figure S44. DSC Plot 1 for ethyl (2-methoxyphenyl)diazoacetate (50)

Sample mass $-3.1 \mathrm{mg}$

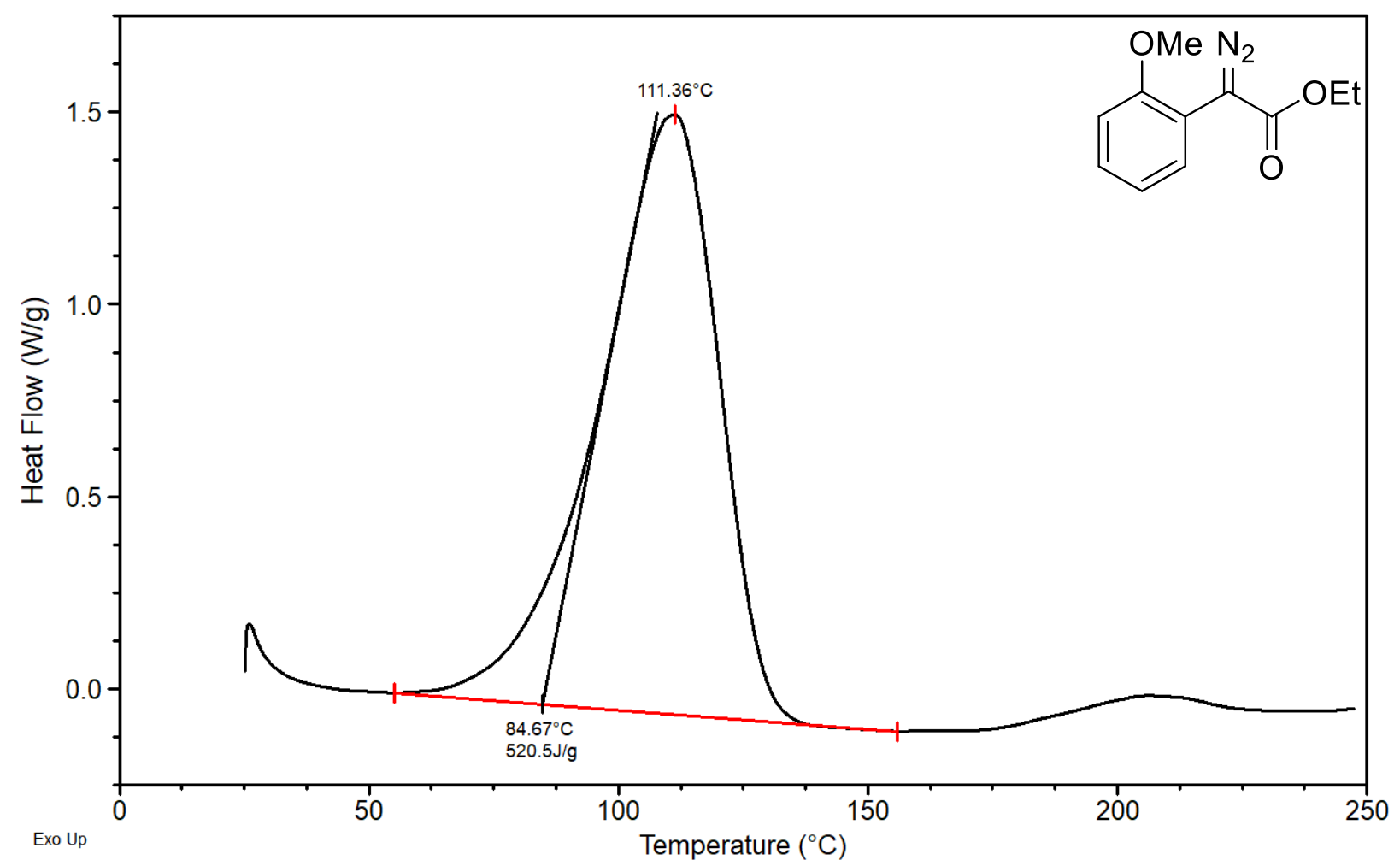

Figure S45. DSC Plot 2 for ethyl (2-methoxyphenyl)diazoacetate (50)

Sample mass $-4.7 \mathrm{mg}$

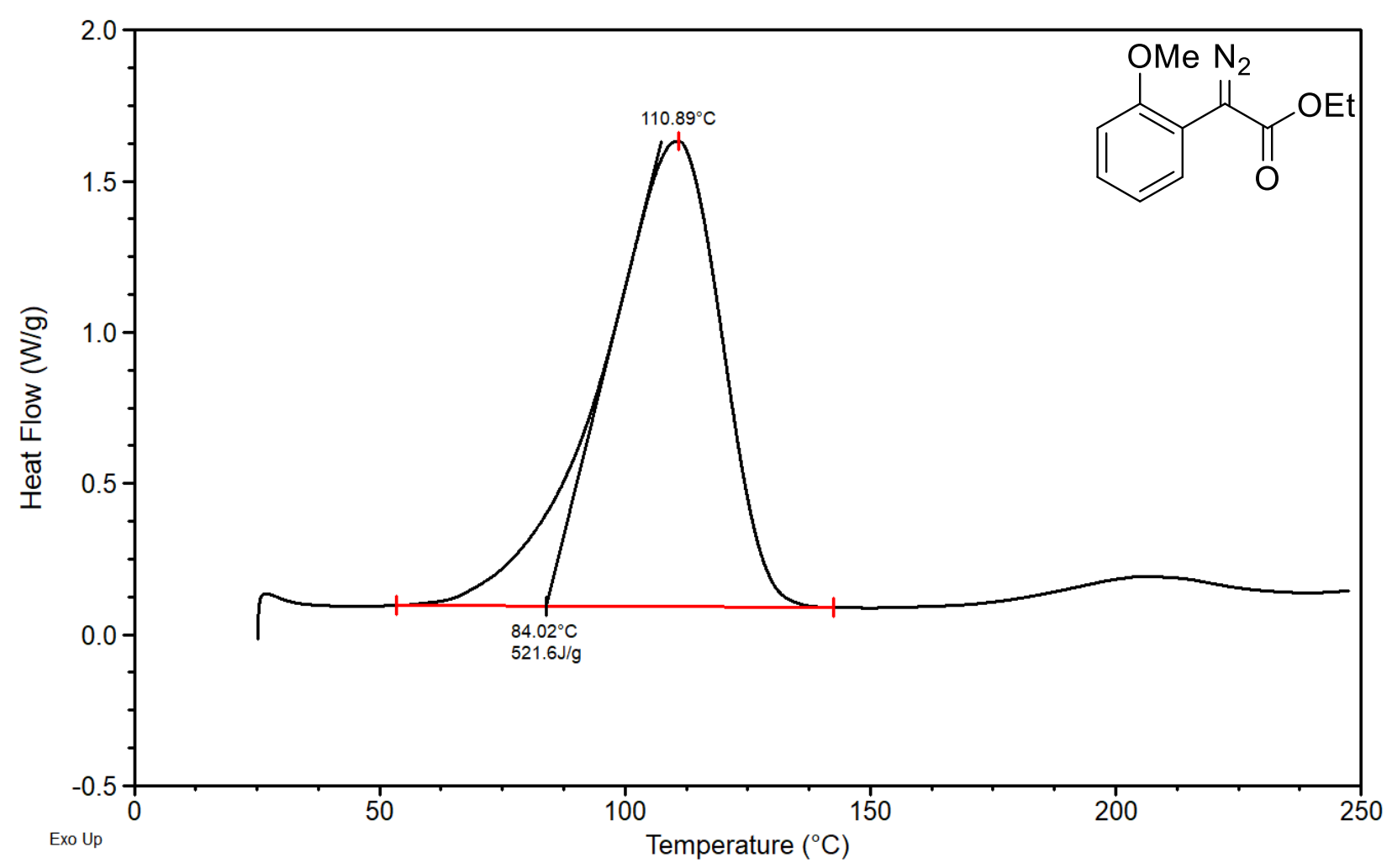


Figure S46. DSC Plot using Method B for ethyl (2-methoxyphenyl)diazoacetate (50) Sample mass $-4.7 \mathrm{mg}$

^exo

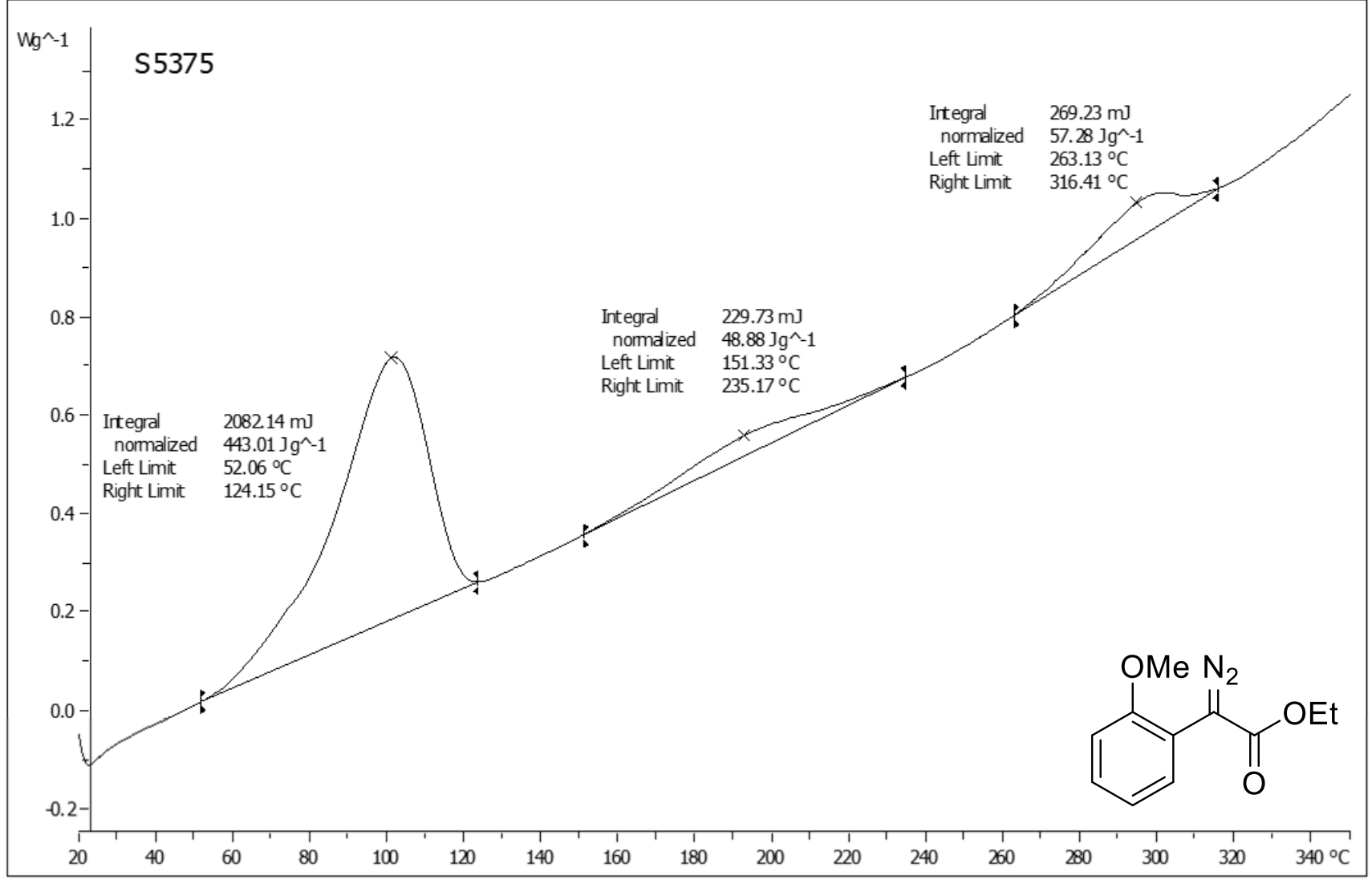


Figure S47. DSC Plot 1 for ethyl (2-fluorophenyl)diazoacetate (51)

Sample mass $-2.7 \mathrm{mg}$

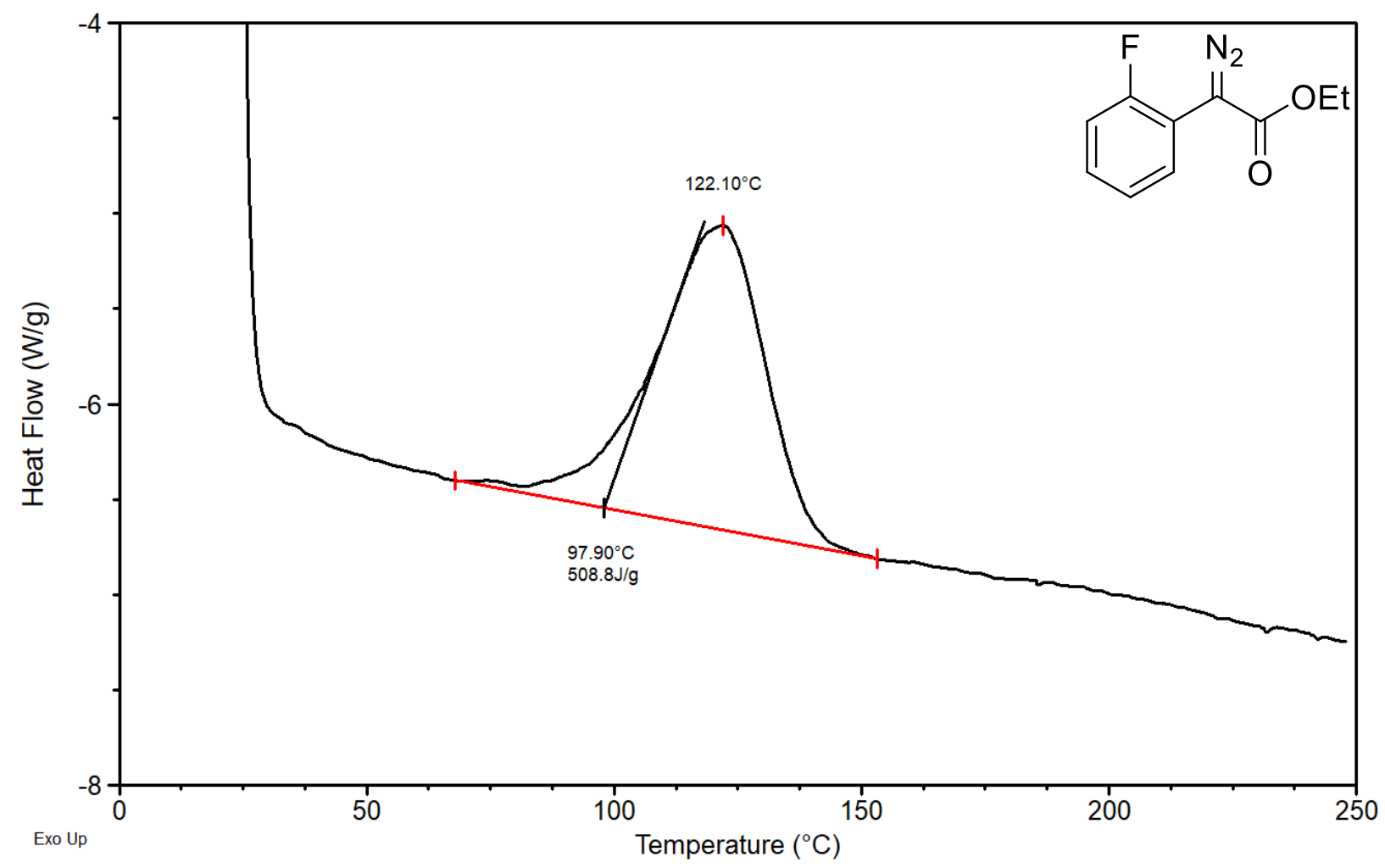

Figure S48. DSC Plot 2 for ethyl (2-fluorophenyl)diazoacetate (51)

Sample mass $-6.1 \mathrm{mg}$

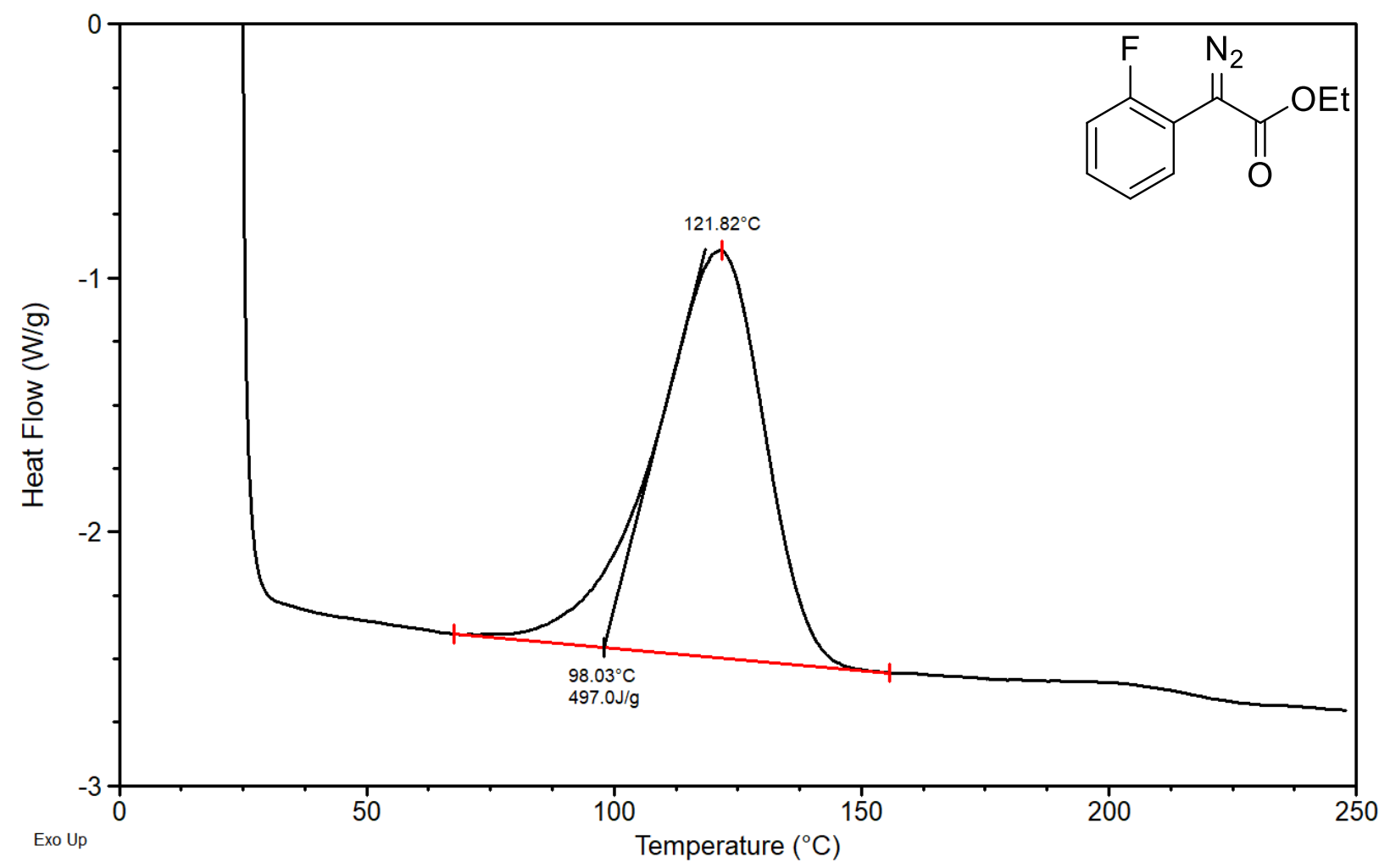


Figure S49. DSC Plot 1 for ethyl (2-chlorophenyl)diazoacetate (52)

Sample mass $-3.4 \mathrm{mg}$

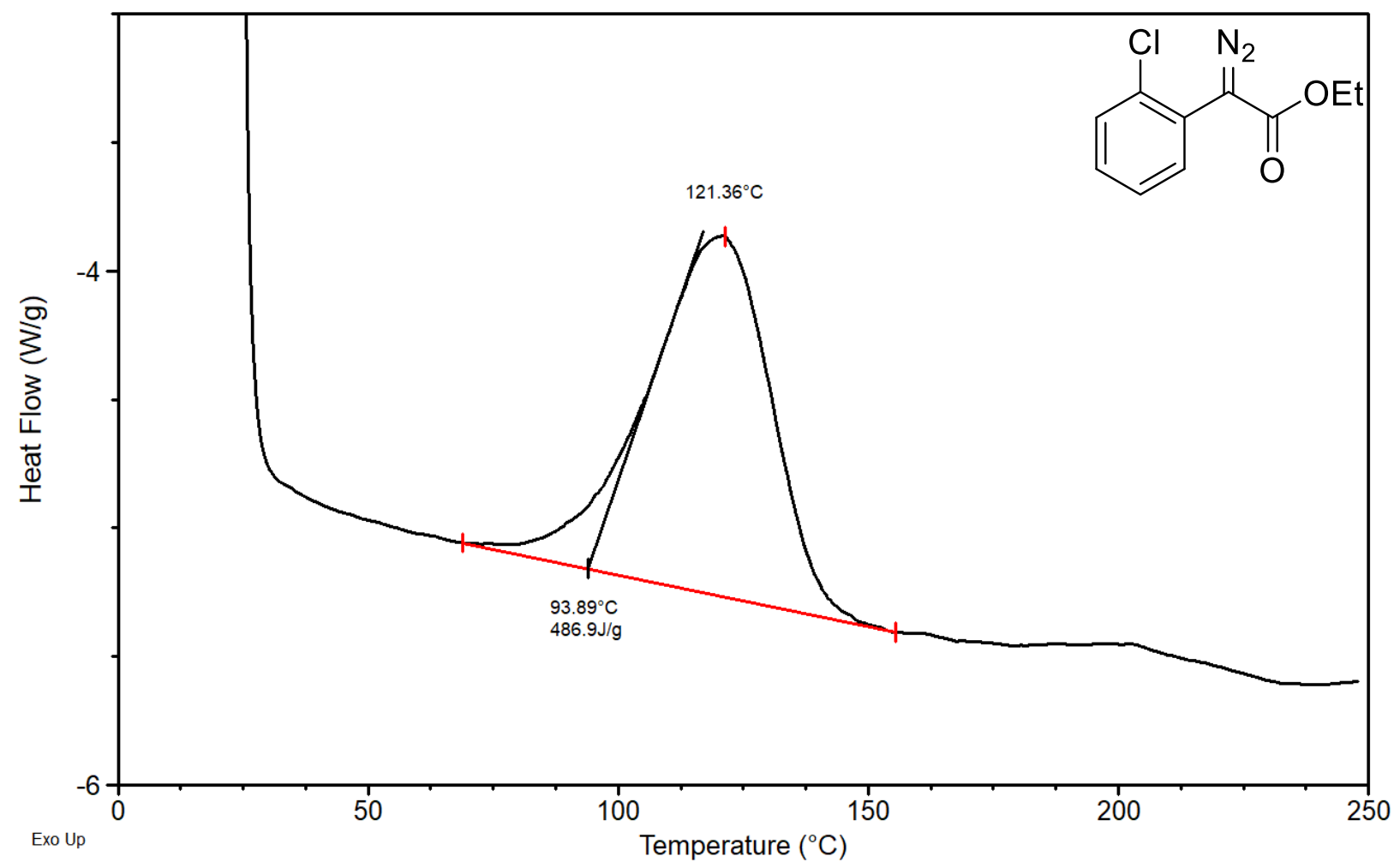

Figure S50. DSC Plot 2 for ethyl (2-chlorophenyl)diazoacetate (52)

Sample mass $-5.5 \mathrm{mg}$

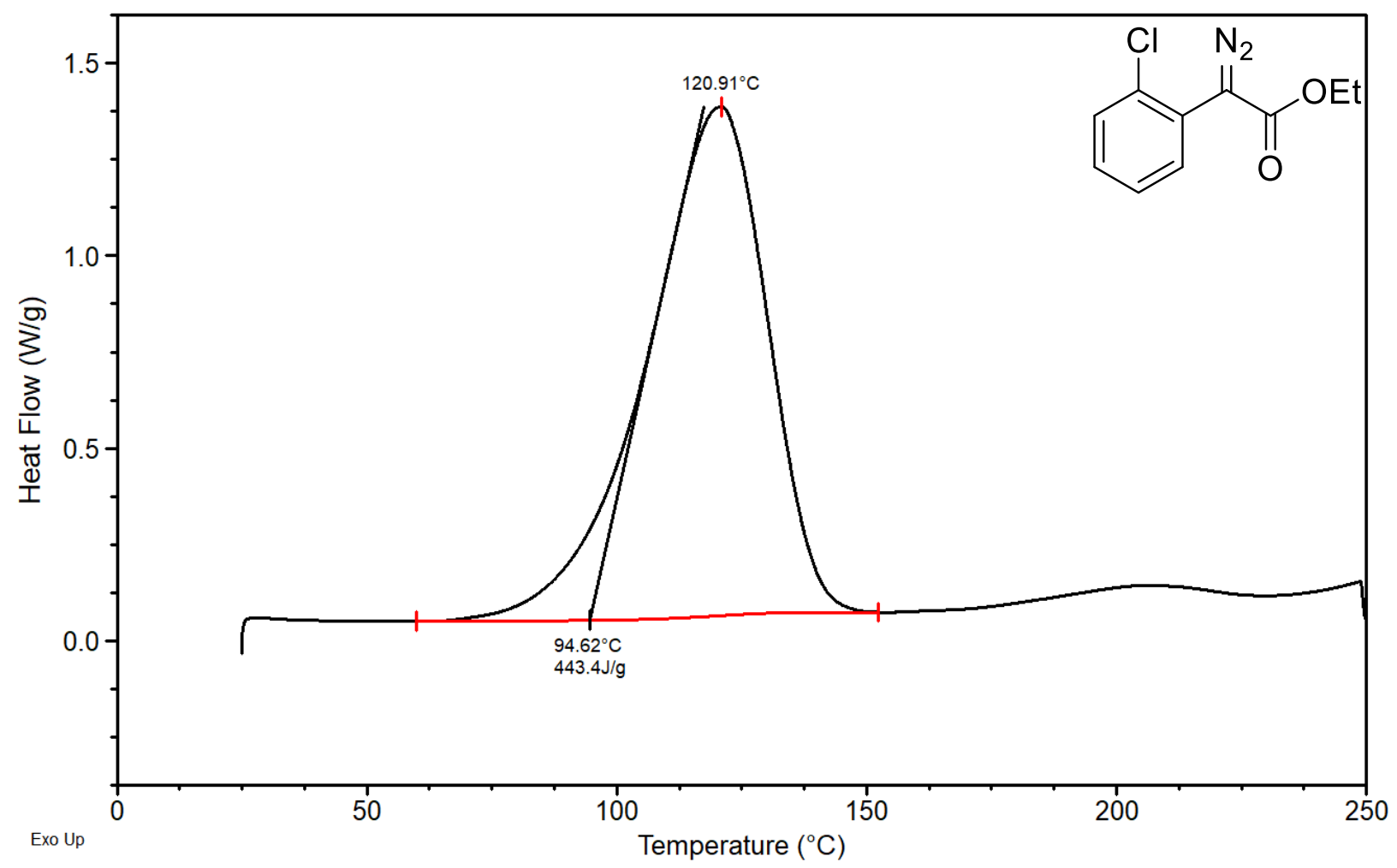


Figure S51. DSC Plot 1 for ethyl (2-trifluoromethylphenyl)diazoacetate (53)

Sample mass $-4.2 \mathrm{mg}$

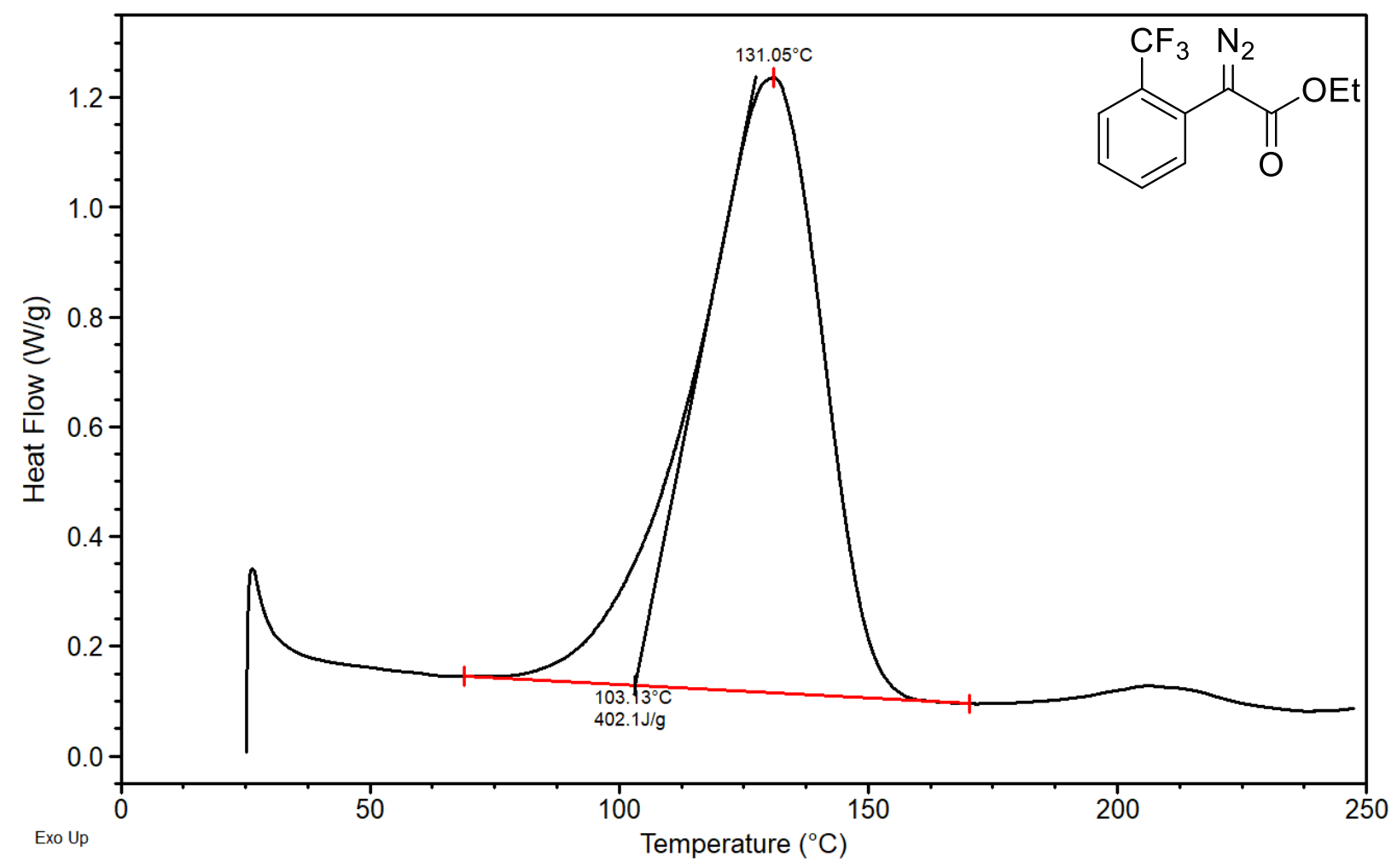

Figure S52. DSC Plot 2 for ethyl (2-trifluoromethylphenyl)diazoacetate (53)

Sample mass $-8.0 \mathrm{mg}$

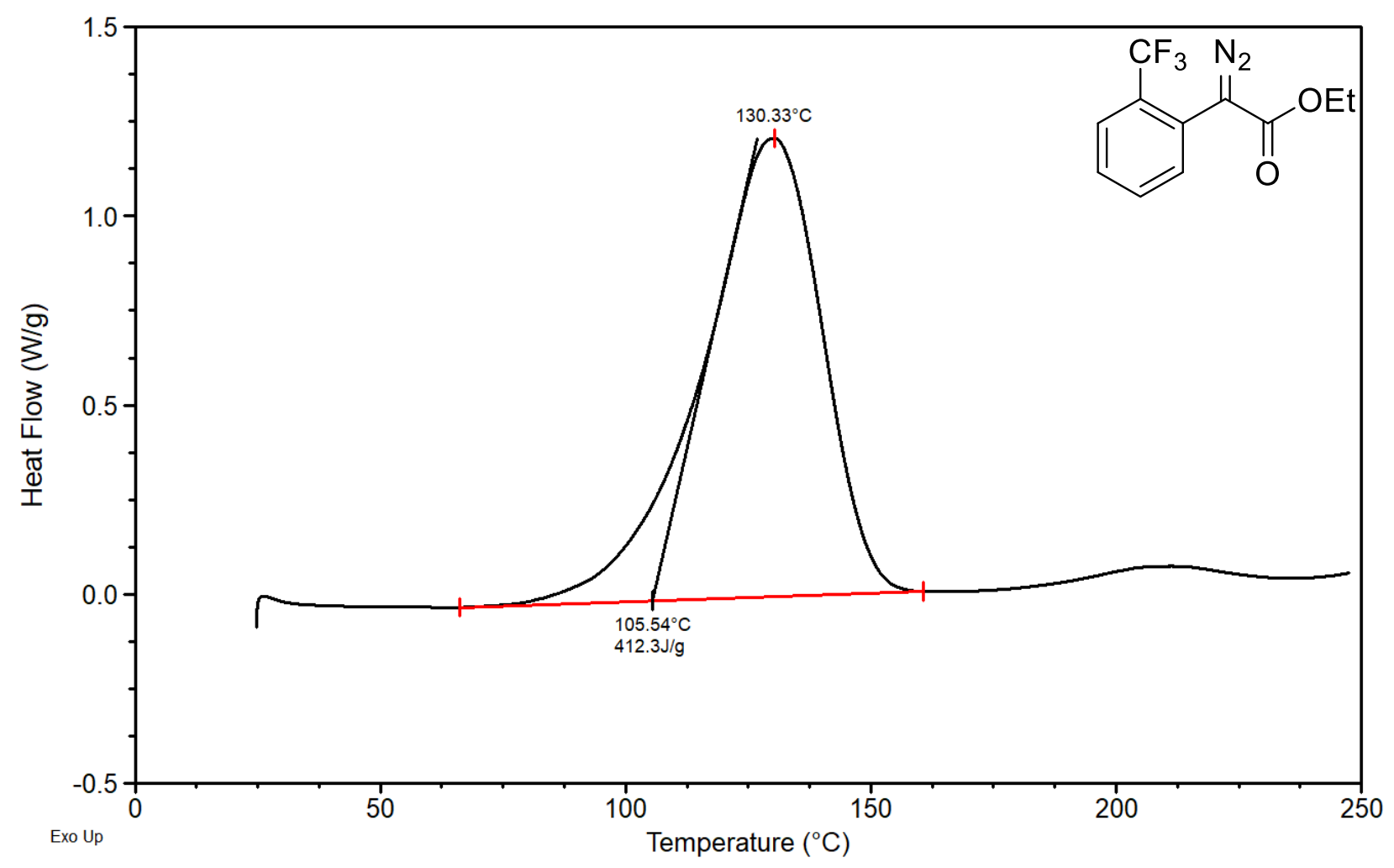


Figure S53. DSC Plot 1 for ethyl (3,4-dichlorophenyl)diazoacetate (54)

Sample mass $-3.9 \mathrm{mg}$

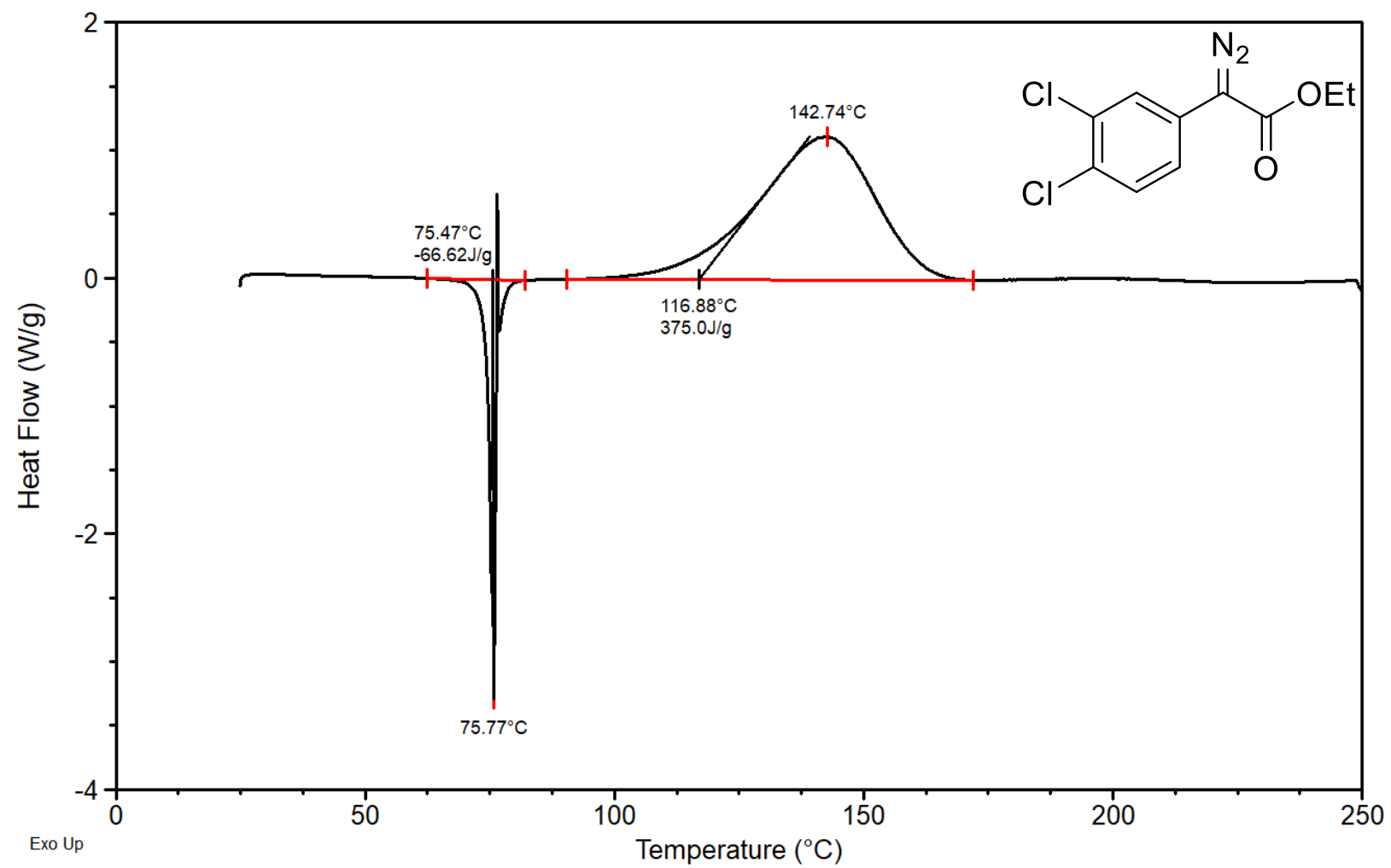

Figure S54. DSC Plot 2 for ethyl (3,4-dichlorophenyl)diazoacetate (54)

Sample mass $-2.7 \mathrm{mg}$

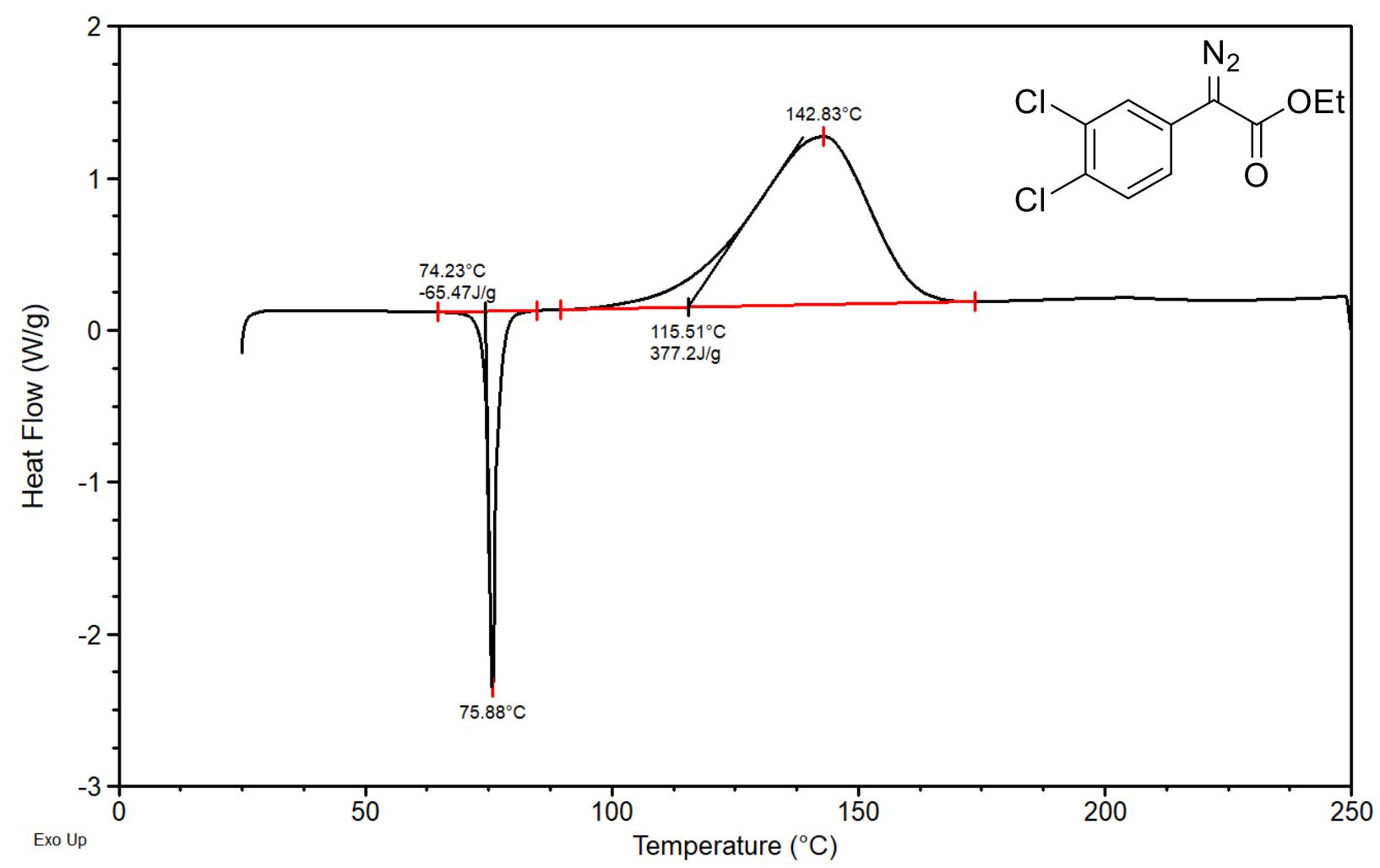


Figure S55. DSC Plot 1 for ethyl (3,4-dimethylphenyl)diazoacetate (55)

Sample mass $-3.1 \mathrm{mg}$

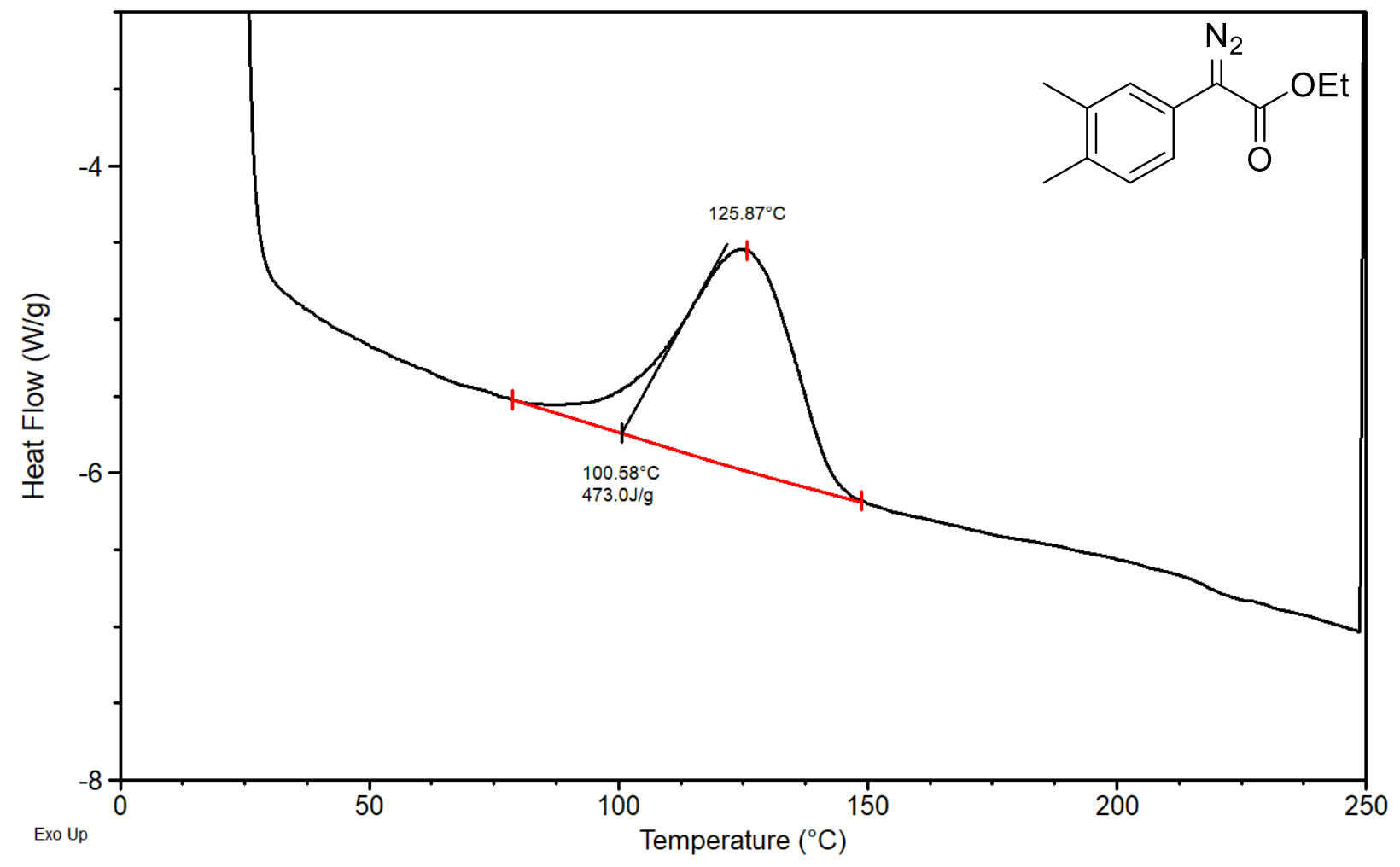

Figure S56. DSC Plot 2 for ethyl (3,4-dimethylphenyl)diazoacetate (55)

Sample mass $-5.4 \mathrm{mg}$

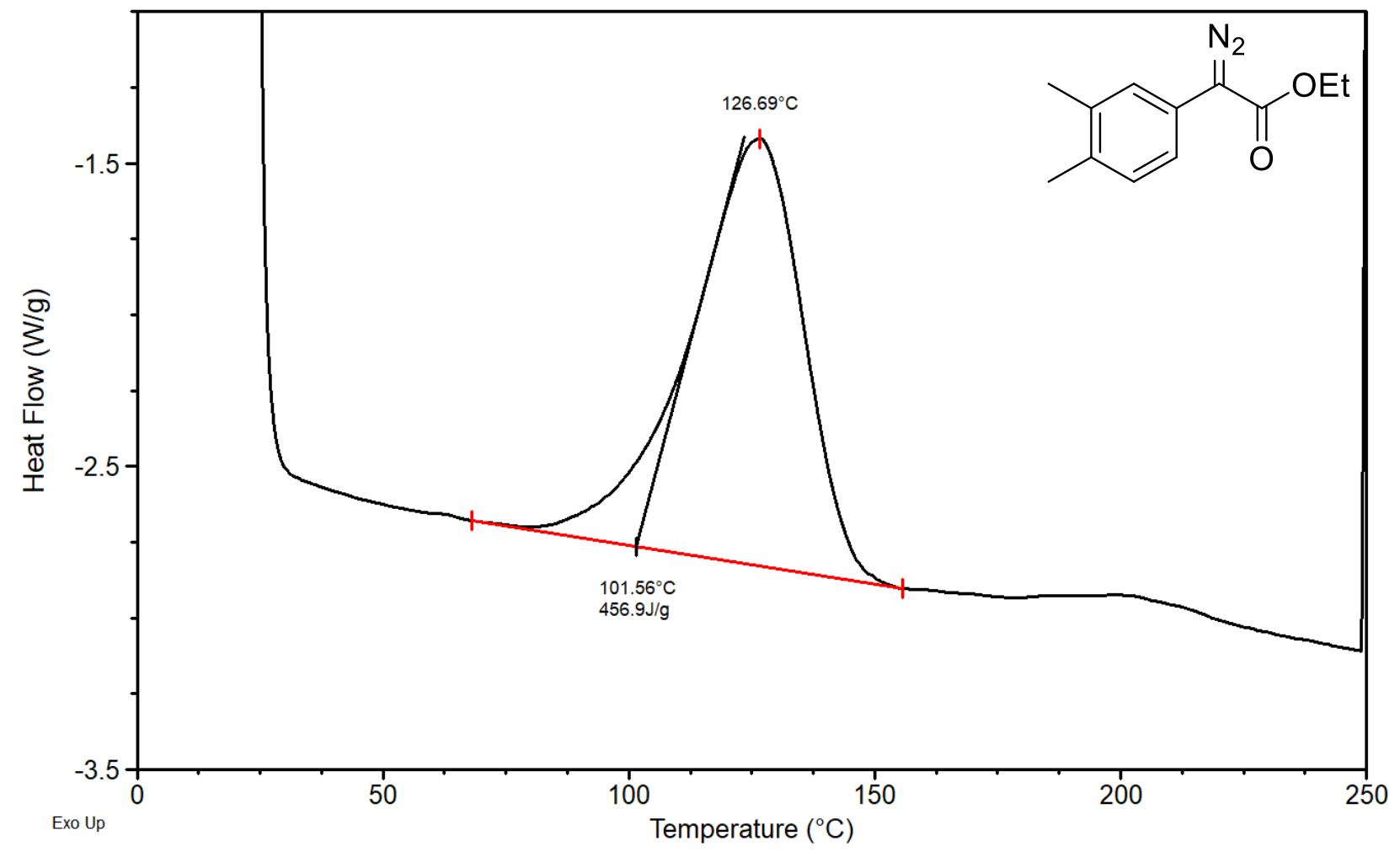


Figure S57. DSC Plot 1 for ethyl (3,4-dimethoxyphenyl)diazoacetate (56)

Sample mass $-1.3 \mathrm{mg}$

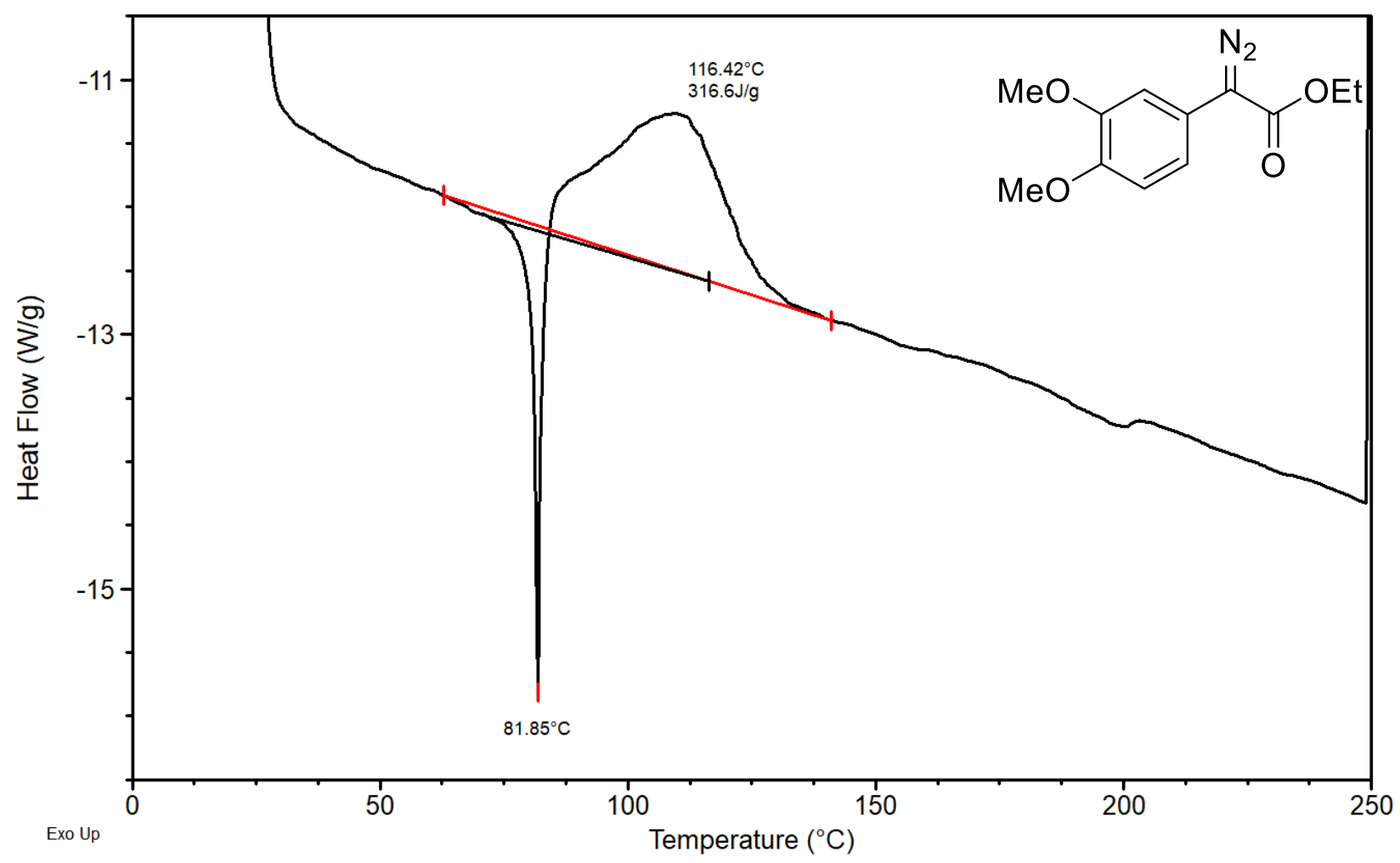

Figure S58. DSC Plot 1 for ethyl (3,4-dimethoxyphenyl)diazoacetate (56) with melting endotherm removed and data points extrapolated

Sample mass $-1.3 \mathrm{mg}$

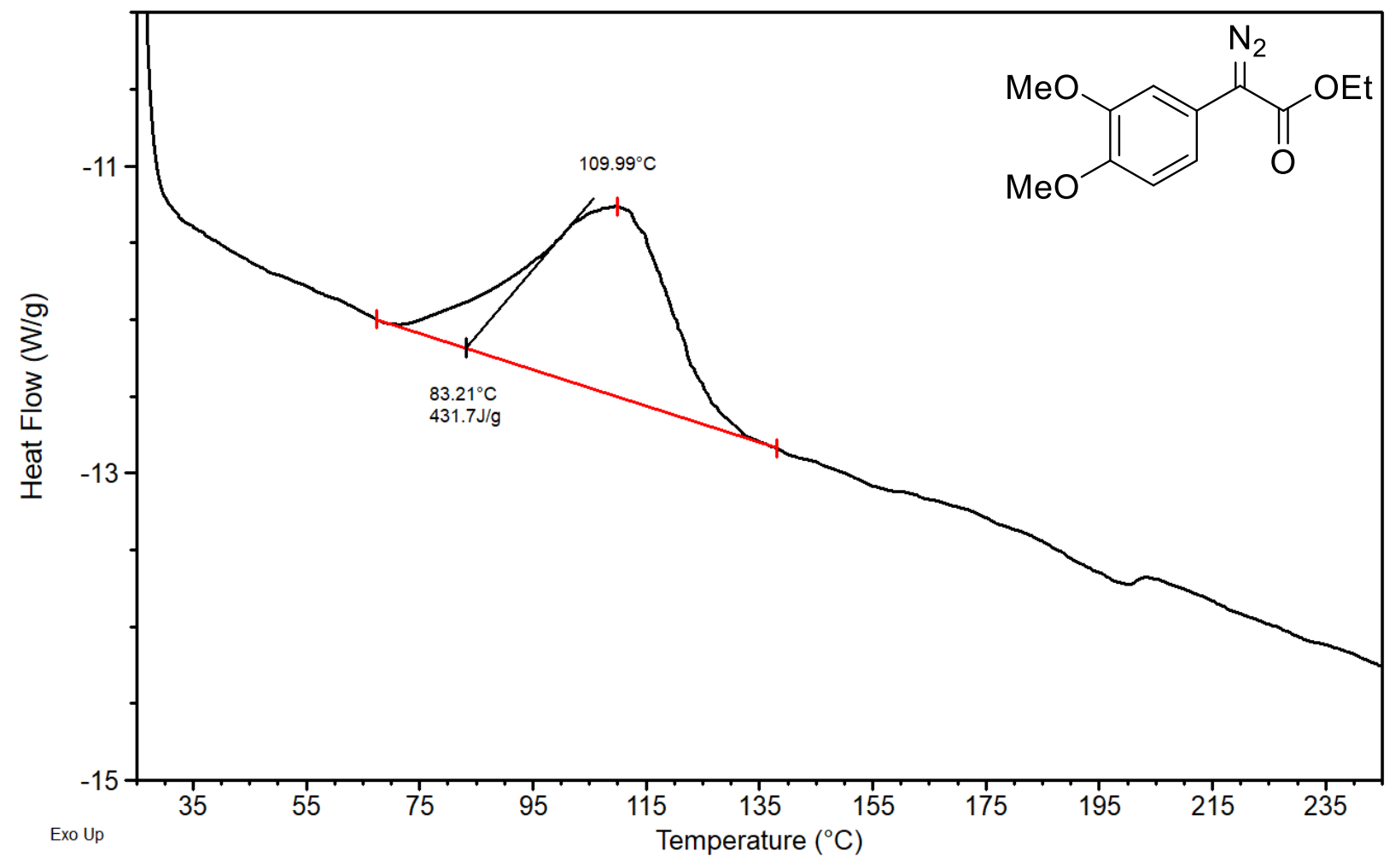


Figure S59. DSC Plot 2 for ethyl (3,4-dimethoxyphenyl)diazoacetate (56)

Sample mass $-2.3 \mathrm{mg}$

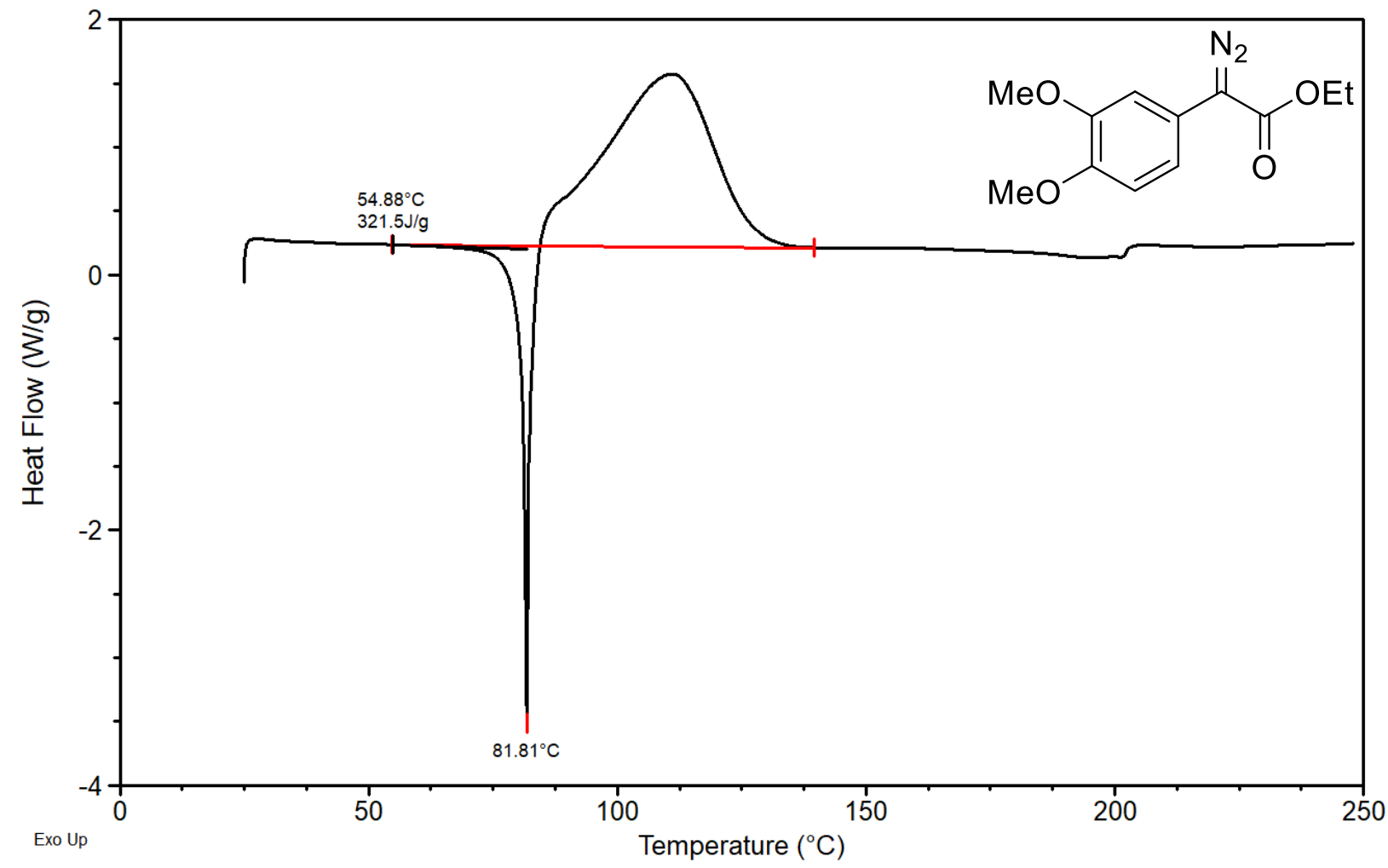

Figure S60. DSC Plot 2 for ethyl (3,4-dimethoxyphenyl)diazoacetate (56) with melting endotherm removed and data points extrapolated

Sample mass $-2.3 \mathrm{mg}$

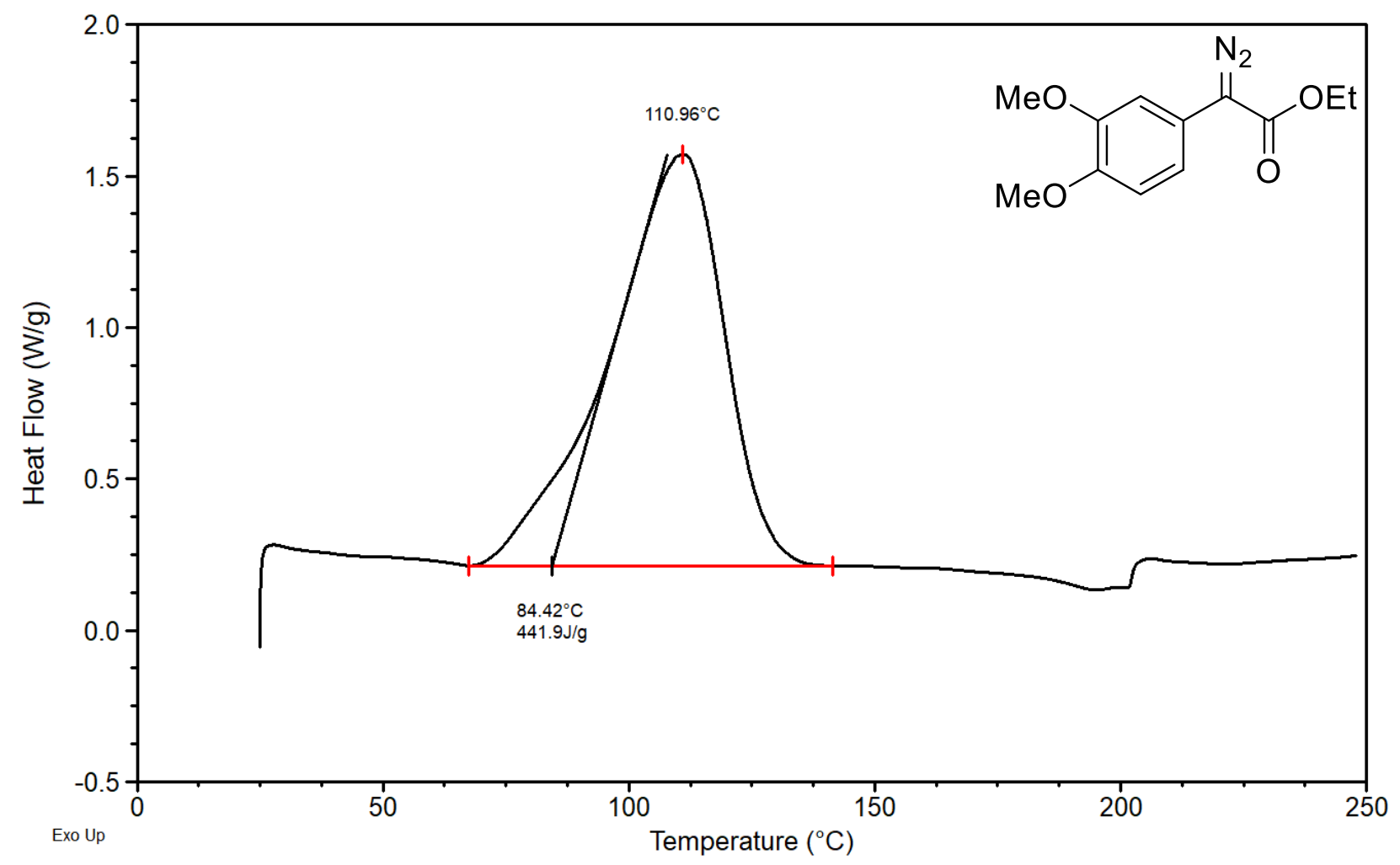


Figure S61. DSC Plot 1 for ethyl (3,4,5-trimethoxyphenyl)diazoacetate (57)

Sample mass $-1.7 \mathrm{mg}$

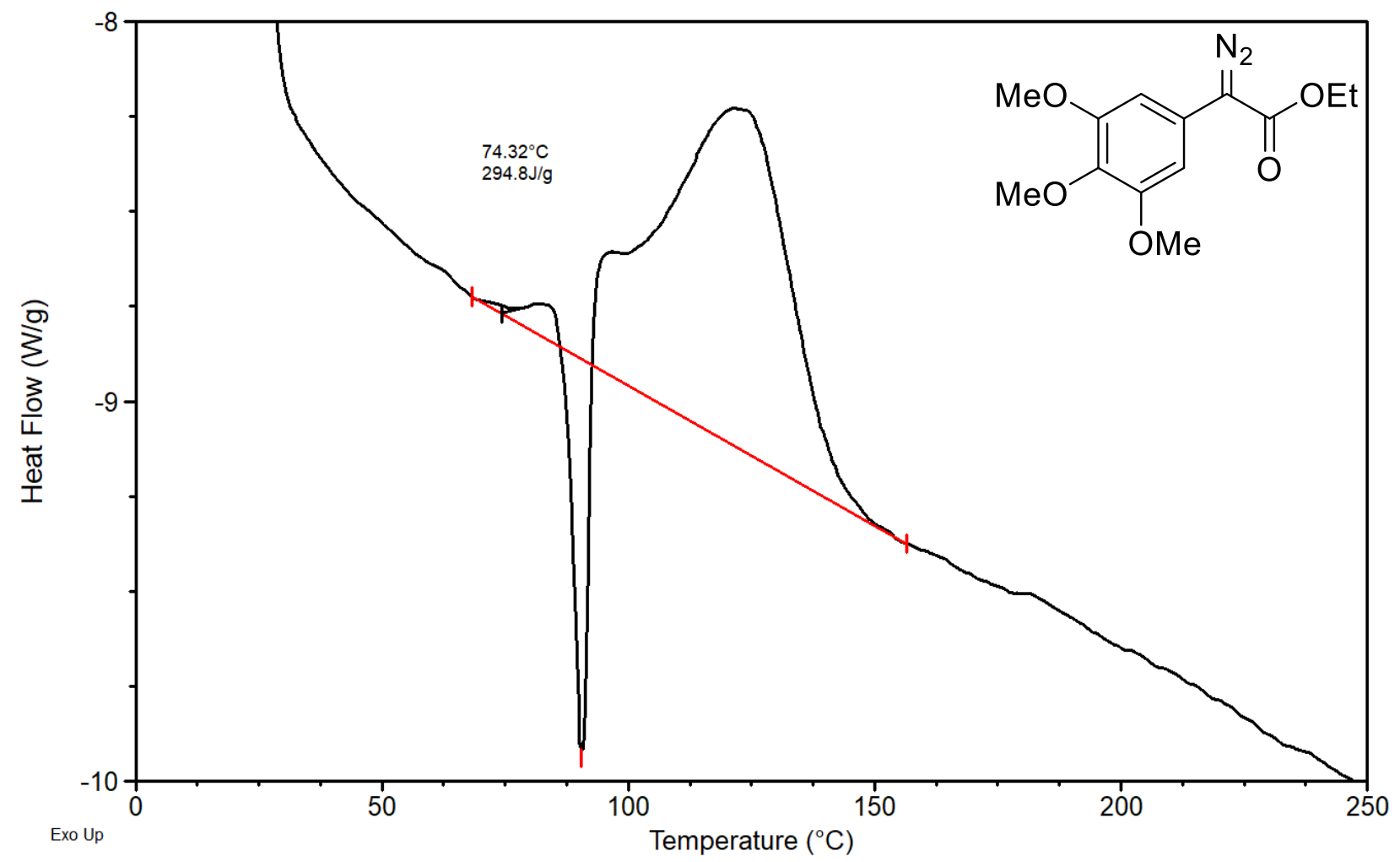

Figure S62. DSC Plot 1 for ethyl (3,4,5-trimethoxyphenyl)diazoacetate (57) with melting endotherm removed and data points extrapolated

Sample mass $-1.7 \mathrm{mg}$

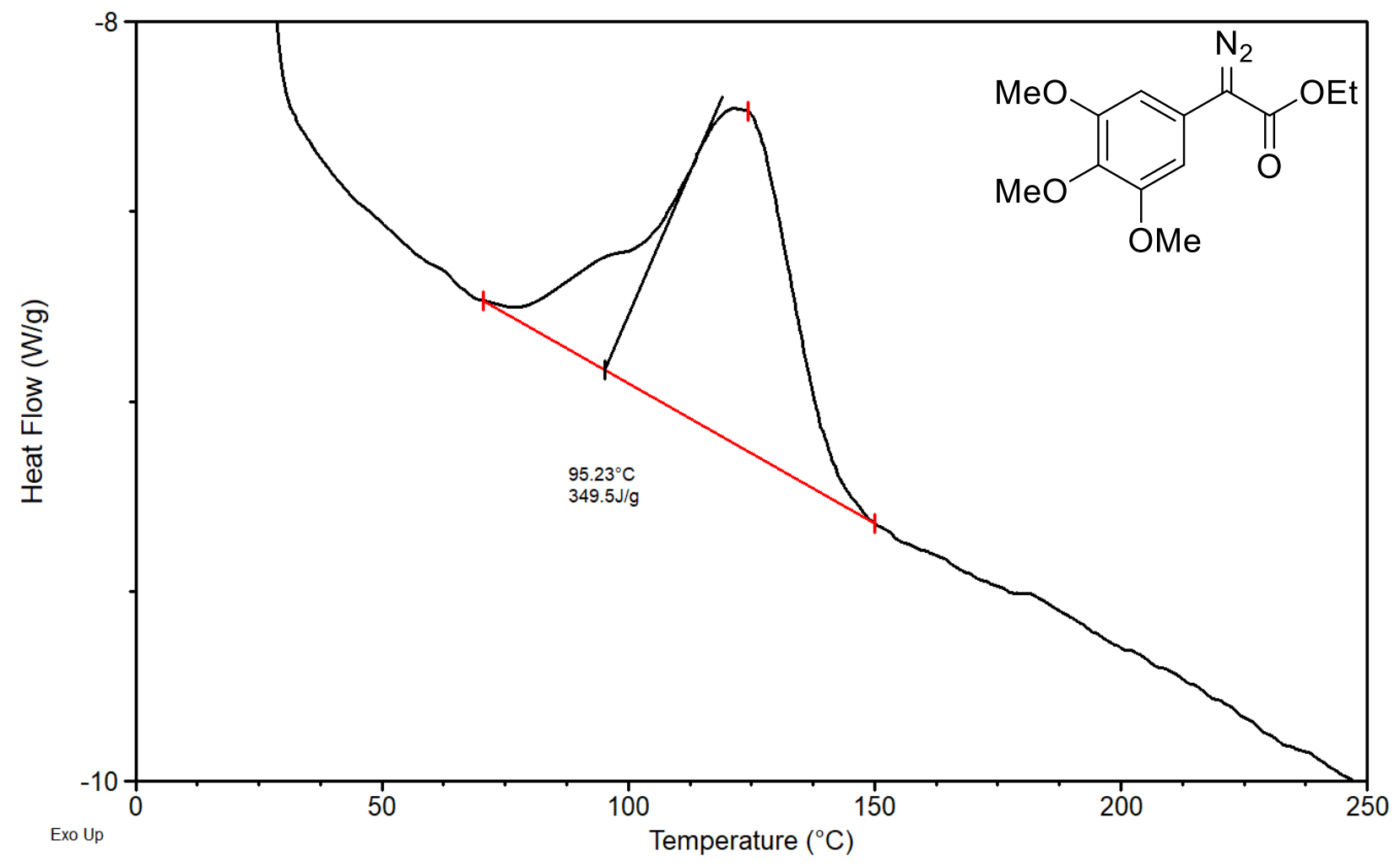


Figure S63. DSC Plot 2 for ethyl (3,4,5-trimethoxyphenyl)diazoacetate (57)

Sample mass $-1.6 \mathrm{mg}$

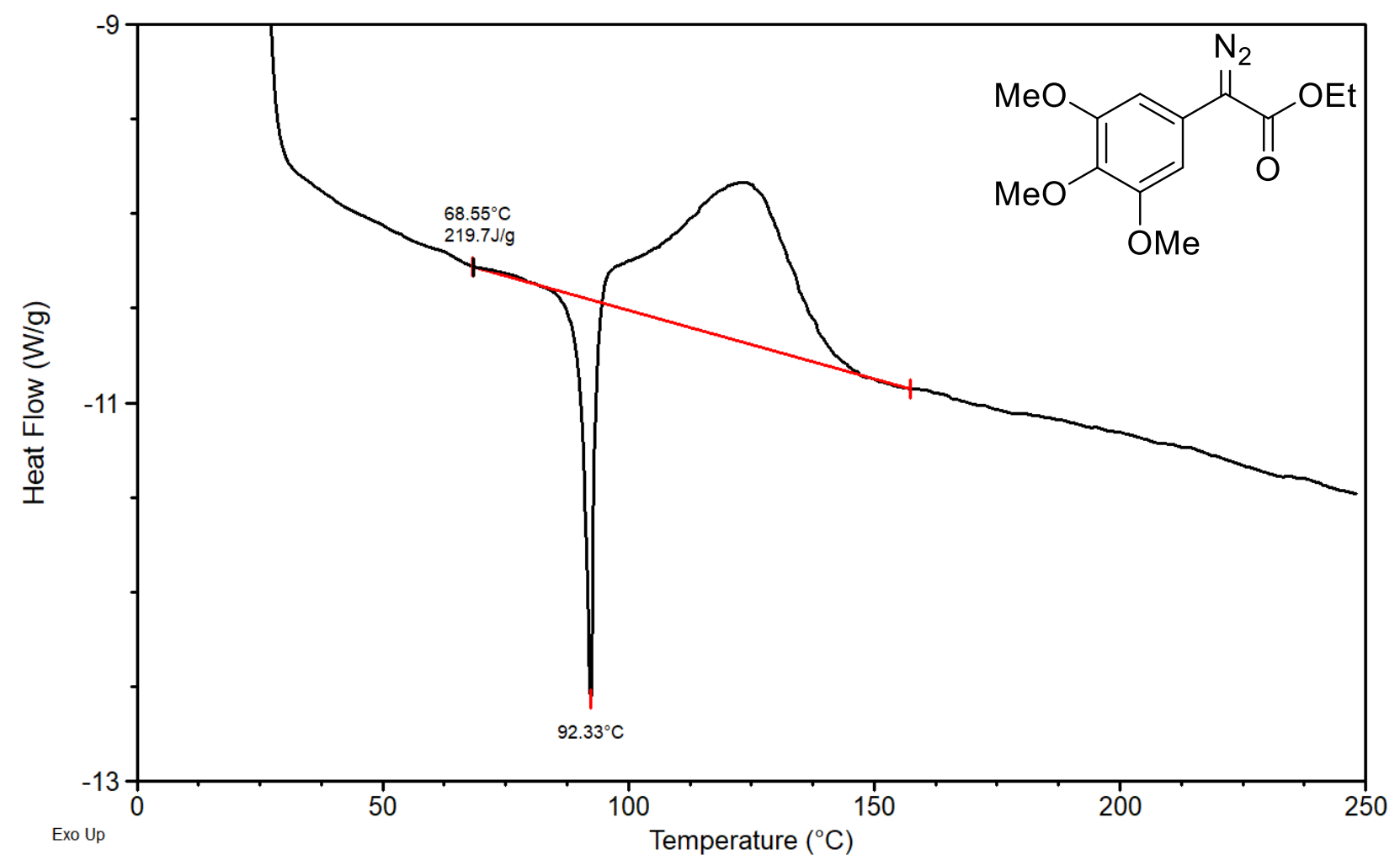

Figure S64. DSC Plot 2 for ethyl (3,4,5-trimethoxyphenyl)diazoacetate (57) with melting endotherm removed and data points extrapolated

Sample mass $-1.6 \mathrm{mg}$

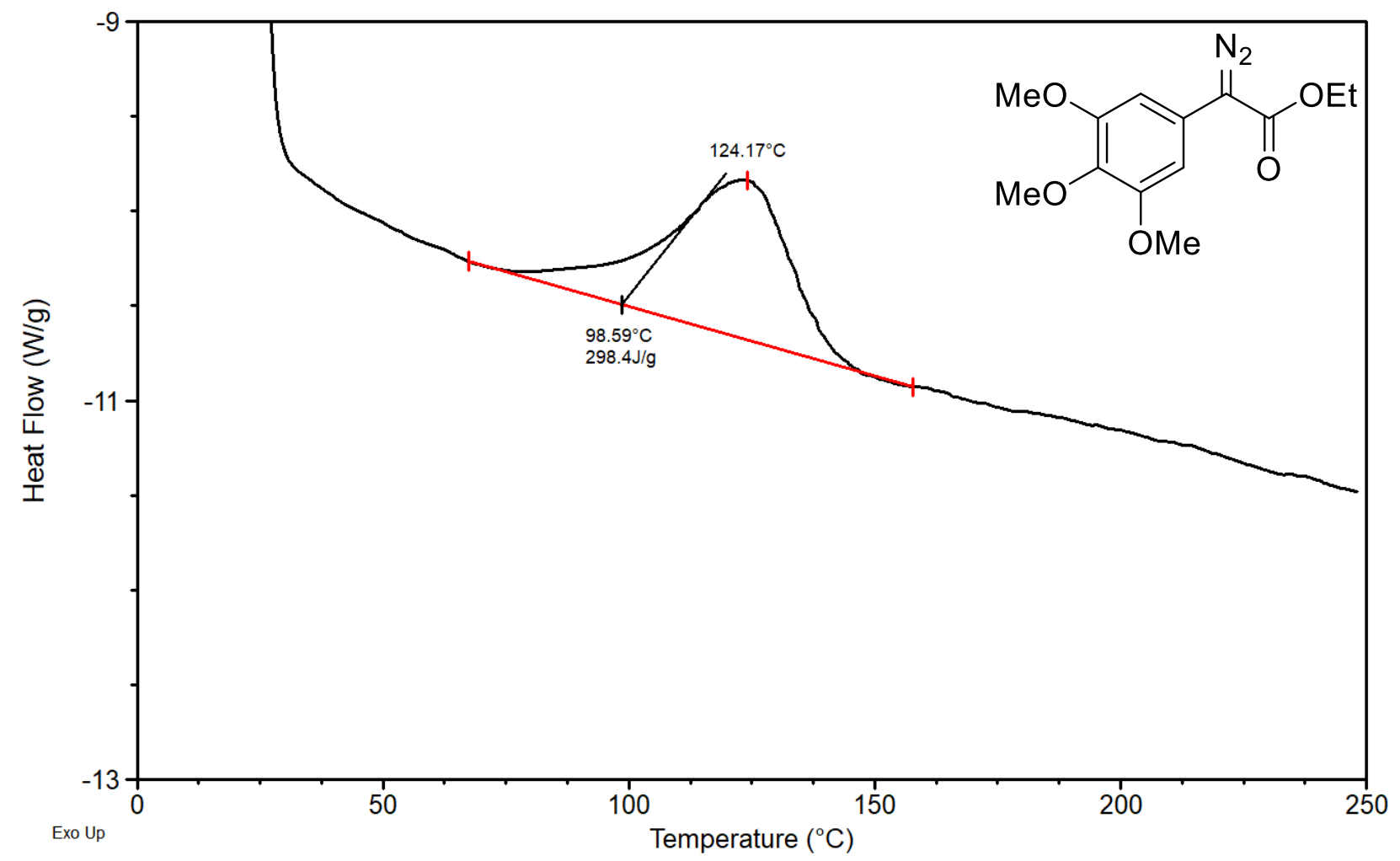


Figure S65. DSC Plot using Method B for ethyl (3,4,5-trimethoxyphenyl)diazoacetate (57) Sample mass $-3.8 \mathrm{mg}$ ^exo

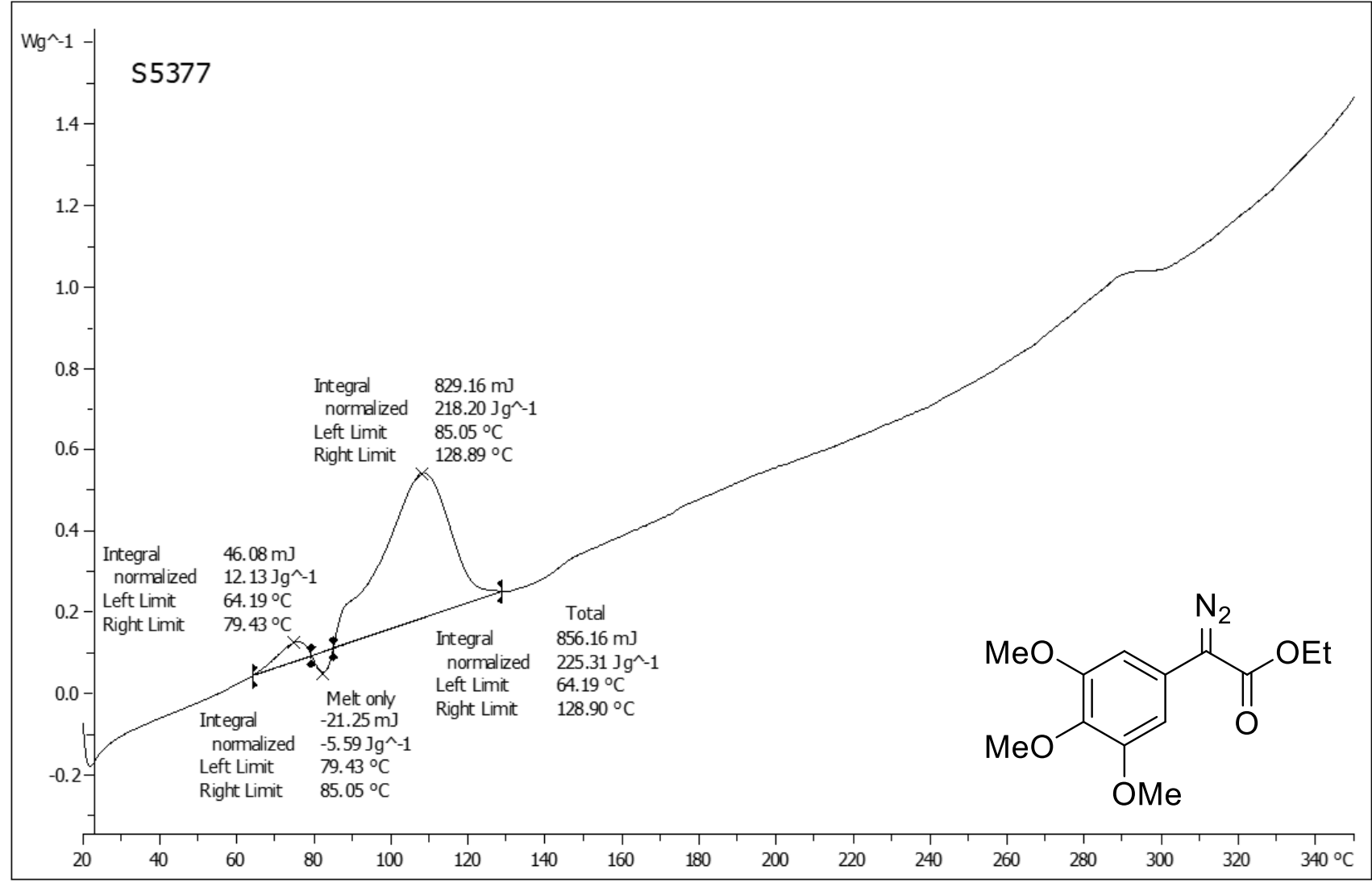


Figure S66. DSC Plot 1 for ethyl (4-phenoxyphenyl)diazoacetate (58)

Sample mass $-4.8 \mathrm{mg}$

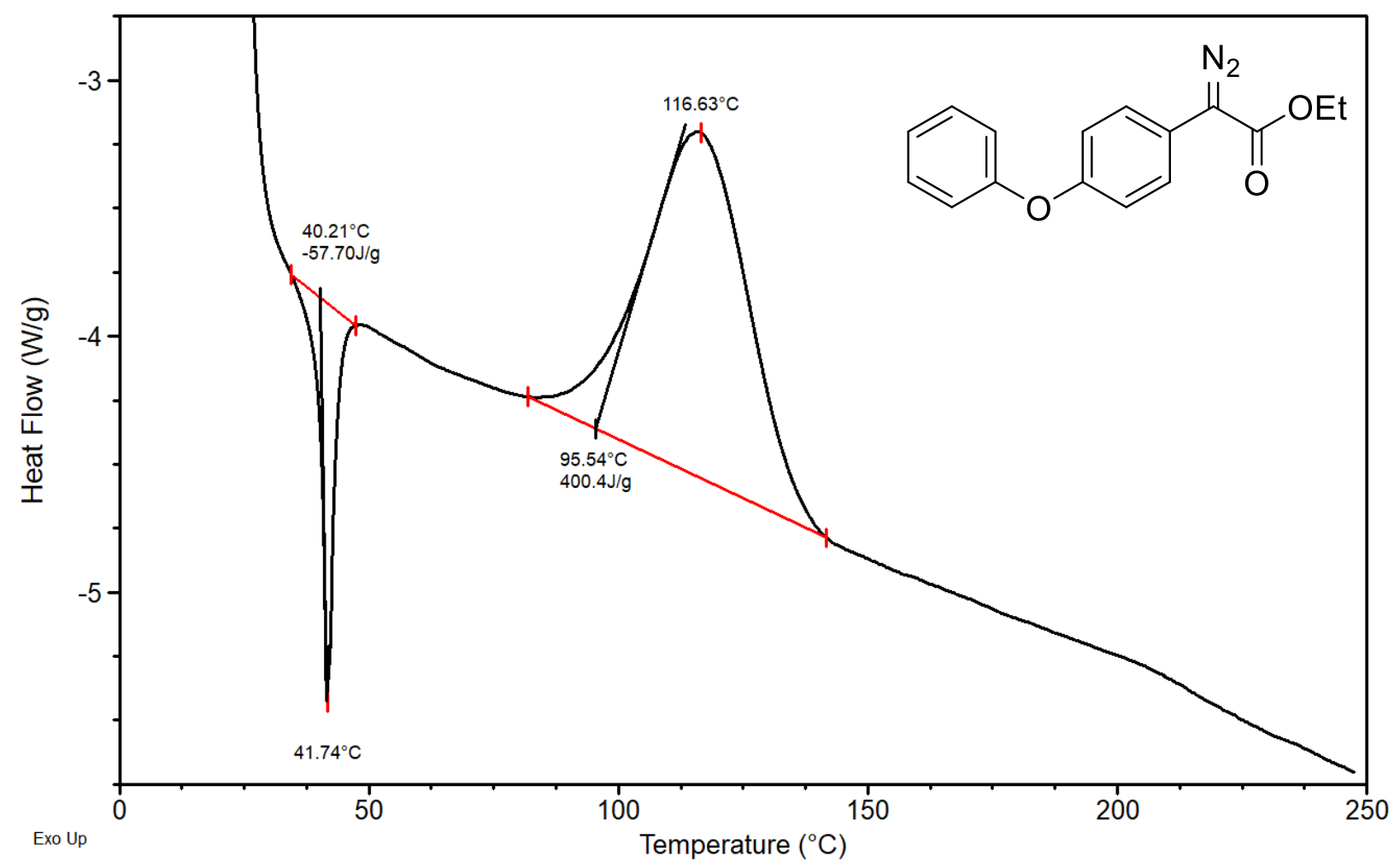

Figure S67. DSC Plot 2 for ethyl (4-phenoxyphenyl)diazoacetate (58)

Sample mass $-5.2 \mathrm{mg}$

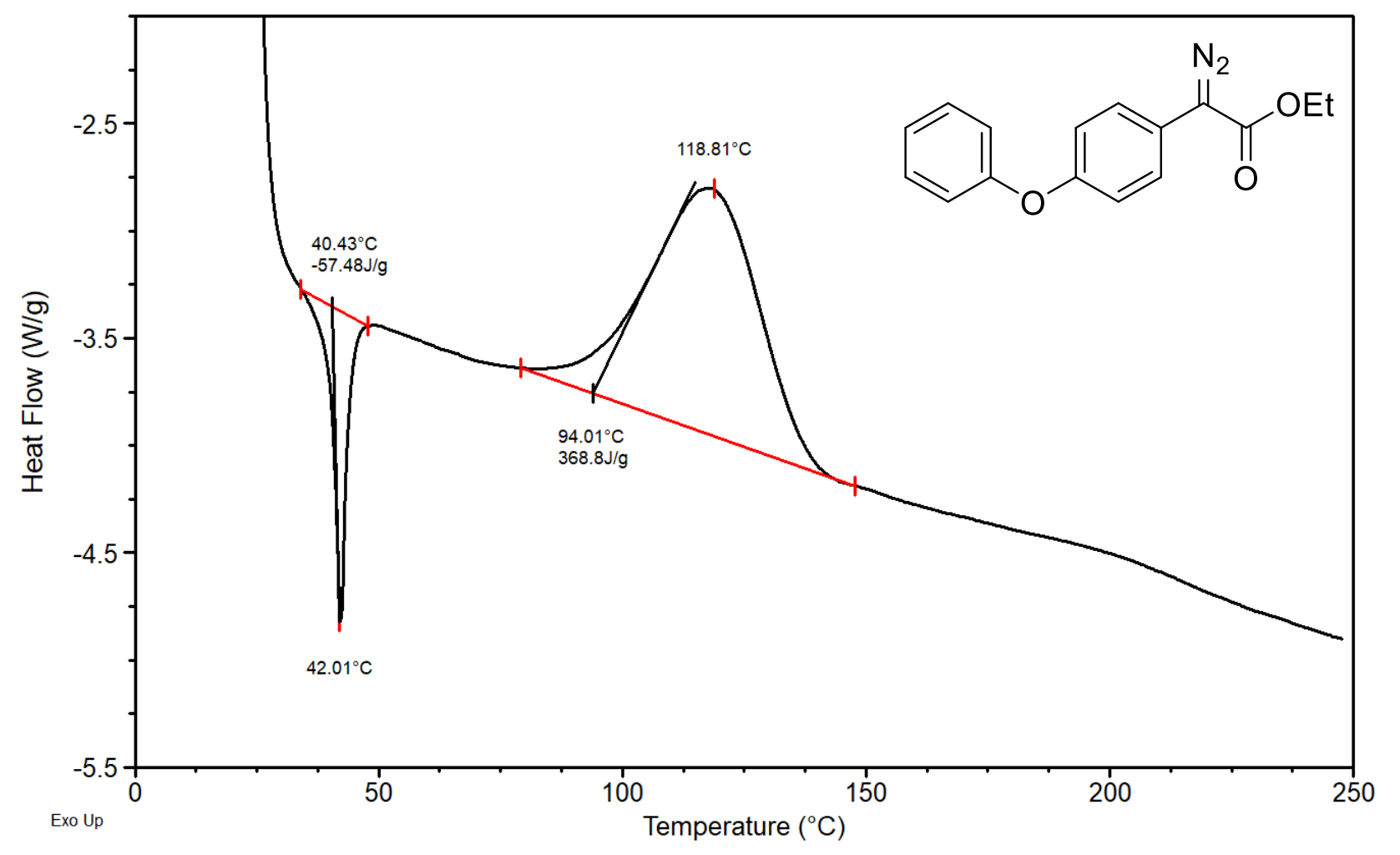


Figure S68. DSC Plot 1 for ethyl ([1,1'-biphenyl]-4-yl)diazoacetate (59)

Sample mass $-5.4 \mathrm{mg}$

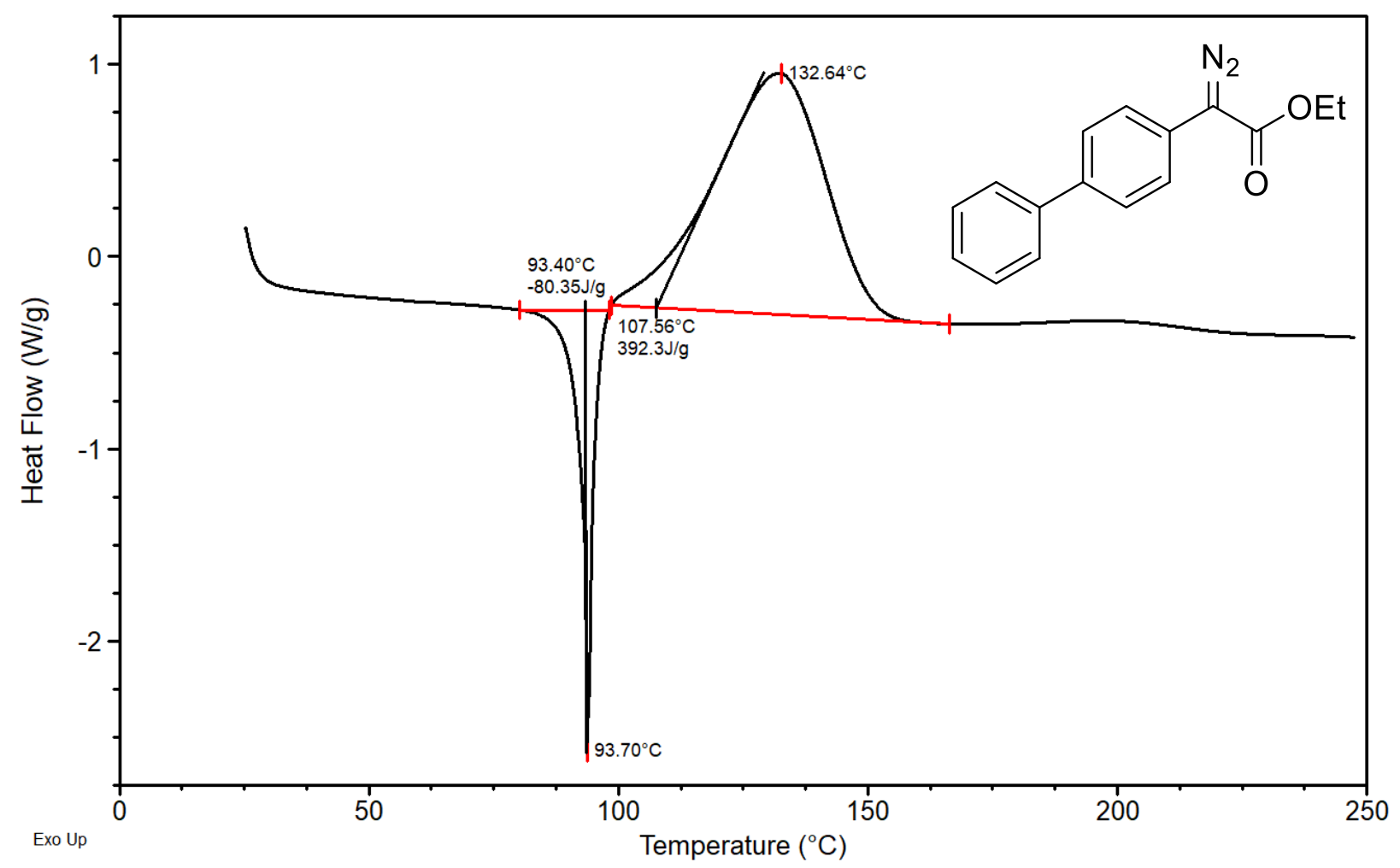

Figure S69. DSC Plot 2 for ethyl ([1,1'-biphenyl]-4-yl)diazoacetate (59)

Sample mass $-5.16 \mathrm{mg}$

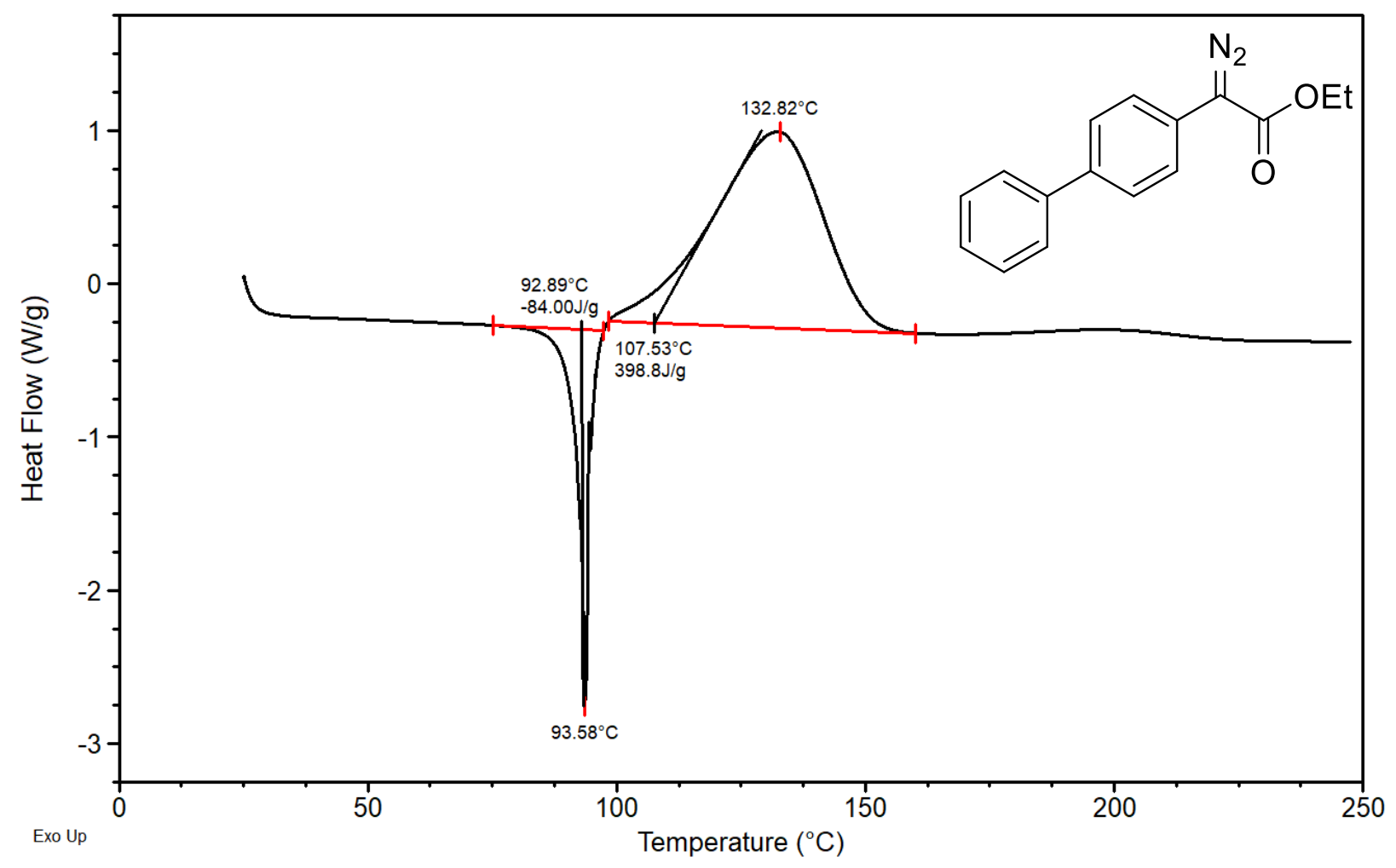


Figure S70. DSC Plot 1 for ethyl (pyridin-4-yl)diazoacetate (60)

Sample mass $-5.5 \mathrm{mg}$

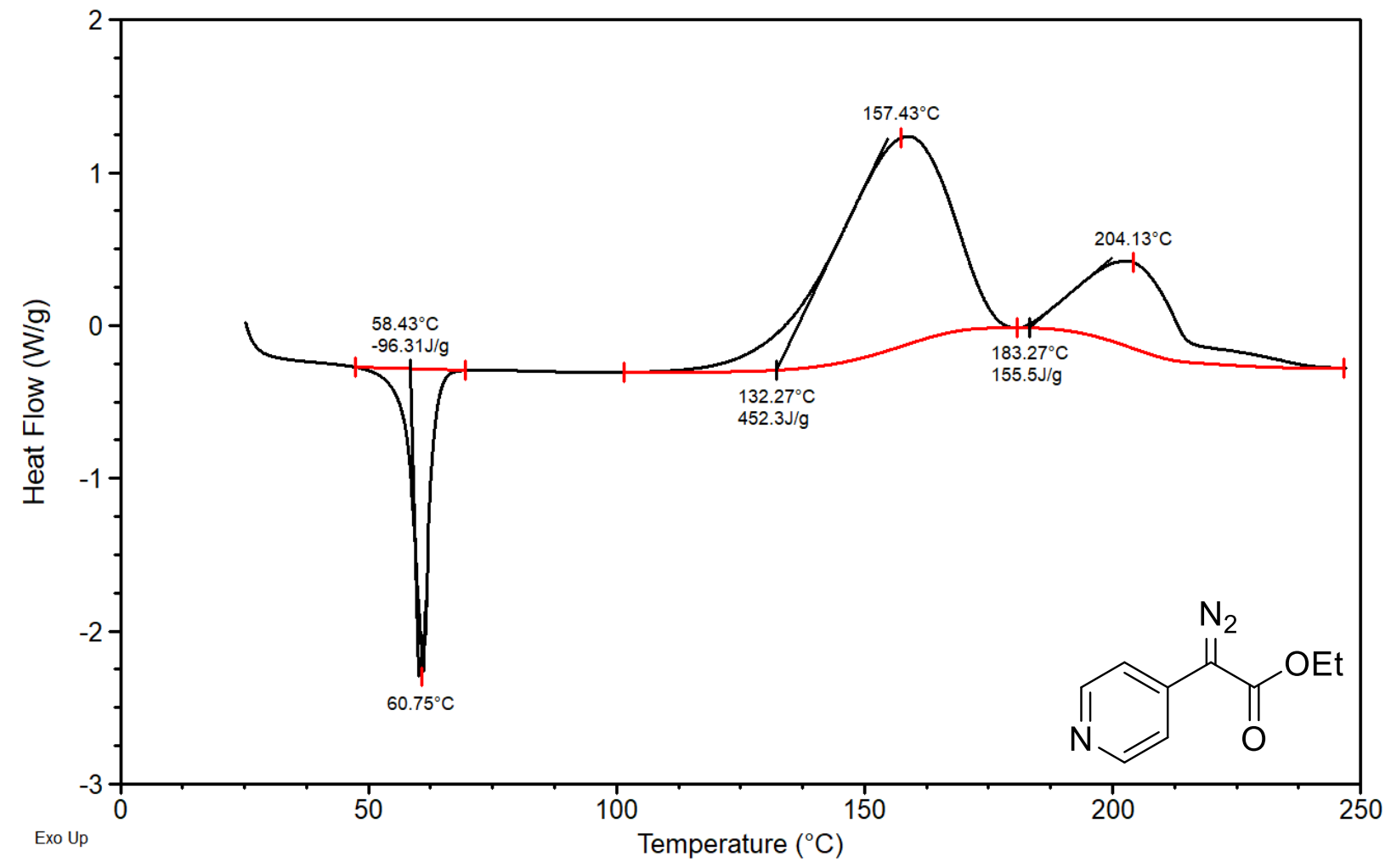

Figure S71. DSC Plot 2 for ethyl (pyridin-4-yl)diazoacetate (60)

Sample mass $-5.5 \mathrm{mg}$

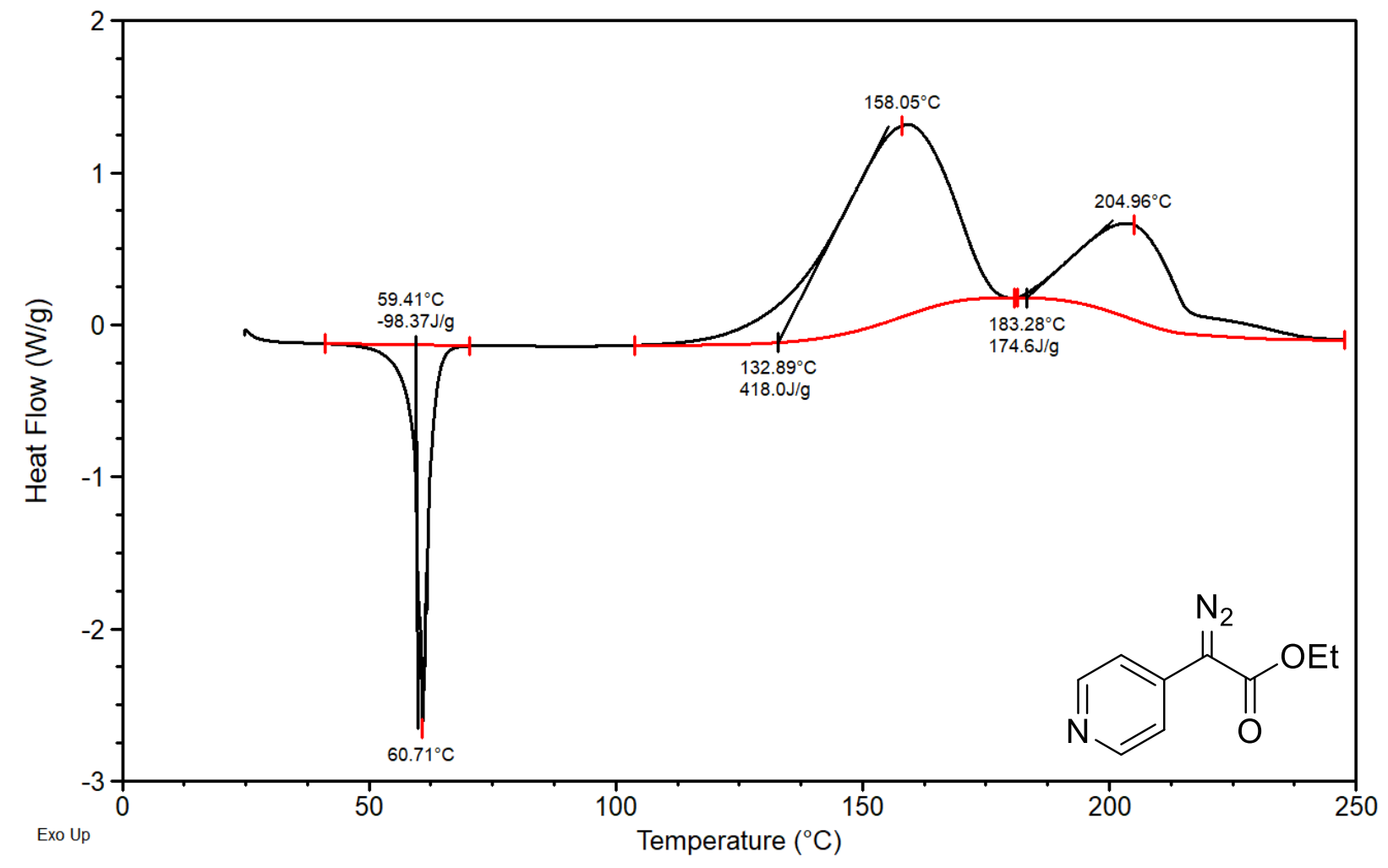


Figure S72. DSC Plot 1 for ethyl (pyridin-3-yl)diazoacetate (61)

Sample mass $-5.3 \mathrm{mg}$

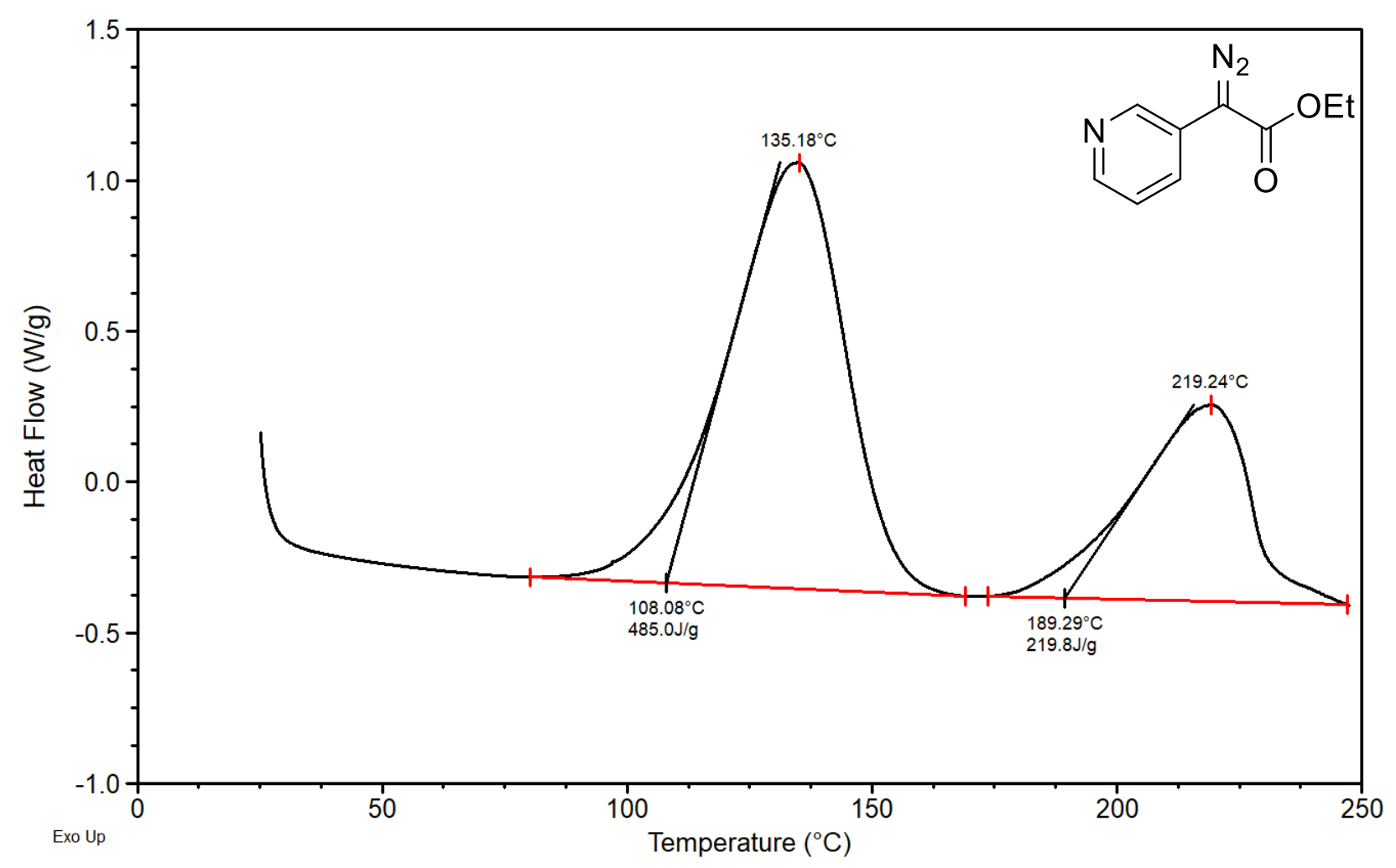

Figure S73. DSC Plot 2 for ethyl (pyridin-3-yl)diazoacetate (61)

Sample mass $-4.7 \mathrm{mg}$

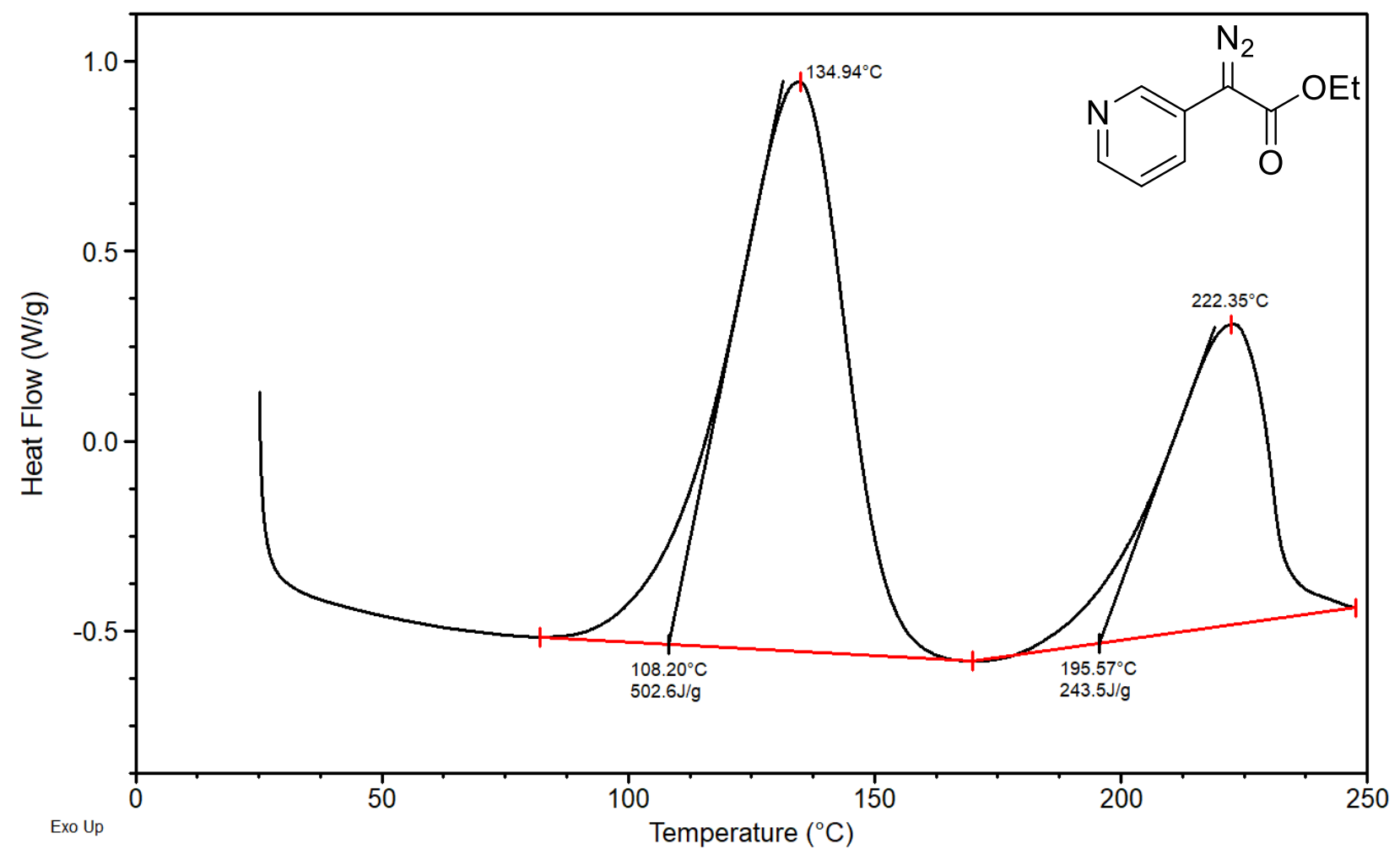


Figure S74. DSC Plot 1 for ethyl (6-chloropyridin-3-yl)diazoacetate (62)

Sample mass $-2.1 \mathrm{mg}$

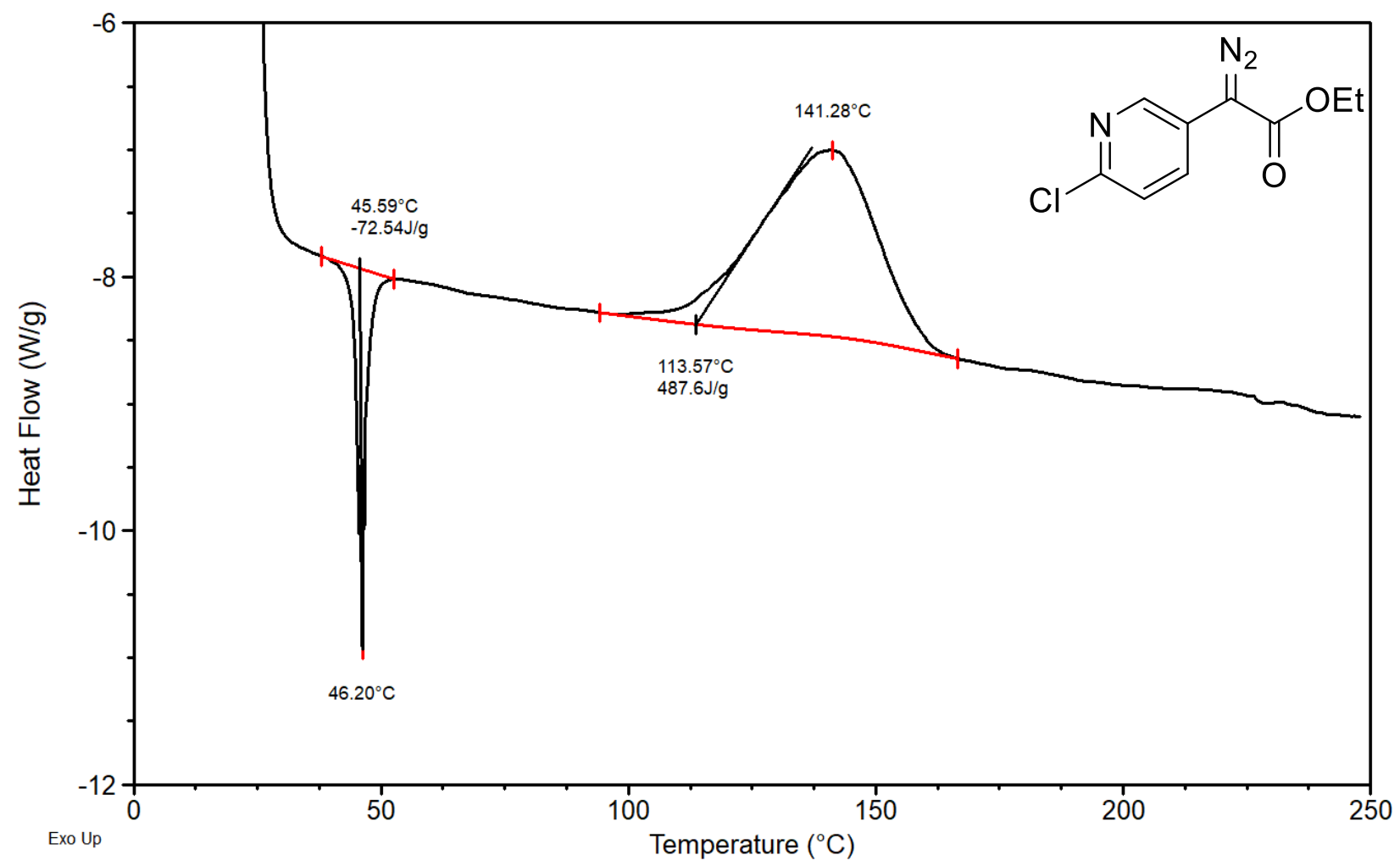

Figure S75. DSC Plot 2 for ethyl (6-chloropyridin-3-yl)diazoacetate (62)

Sample mass $-1.2 \mathrm{mg}$

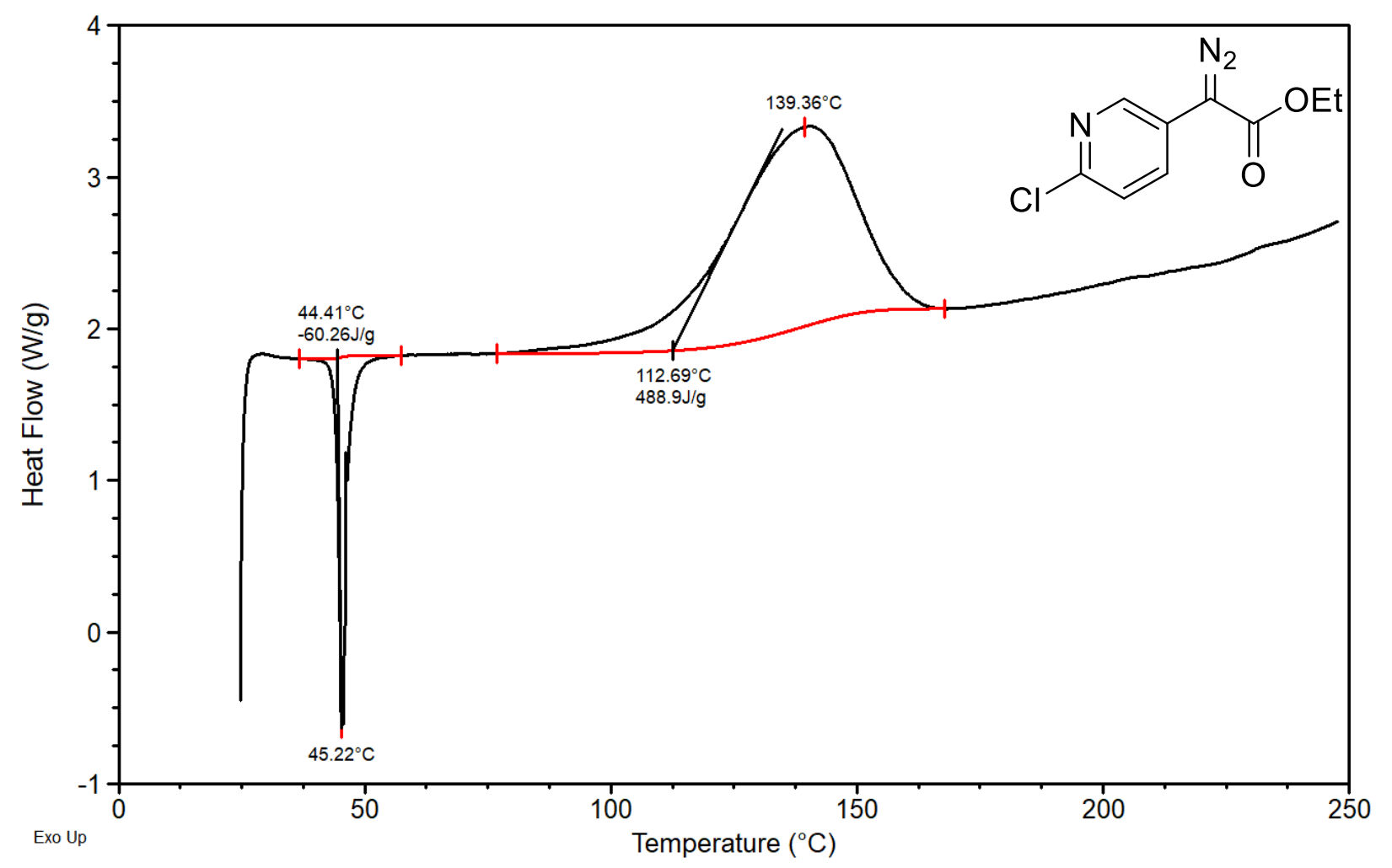


Figure S76. DSC Plot 1 for ethyl [1,2,3]triazolo[1,5-a]pyridine-3-carboxylate (63) Sample mass $-4.8 \mathrm{mg}$

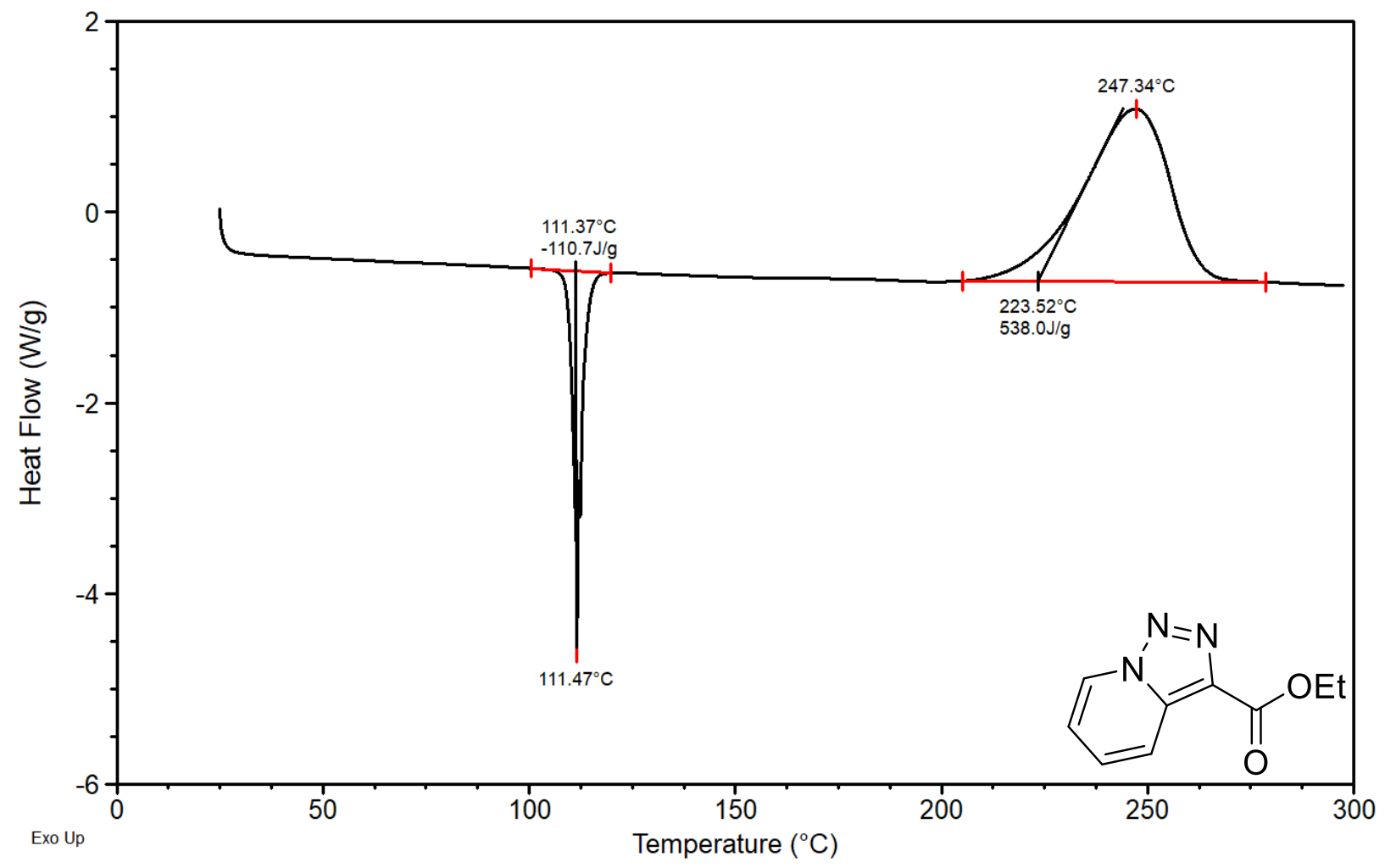

Figure S77. DSC Plot 2 for ethyl [1,2,3]triazolo[1,5-a]pyridine-3-carboxylate (63)

Sample mass $-5.1 \mathrm{mg}$

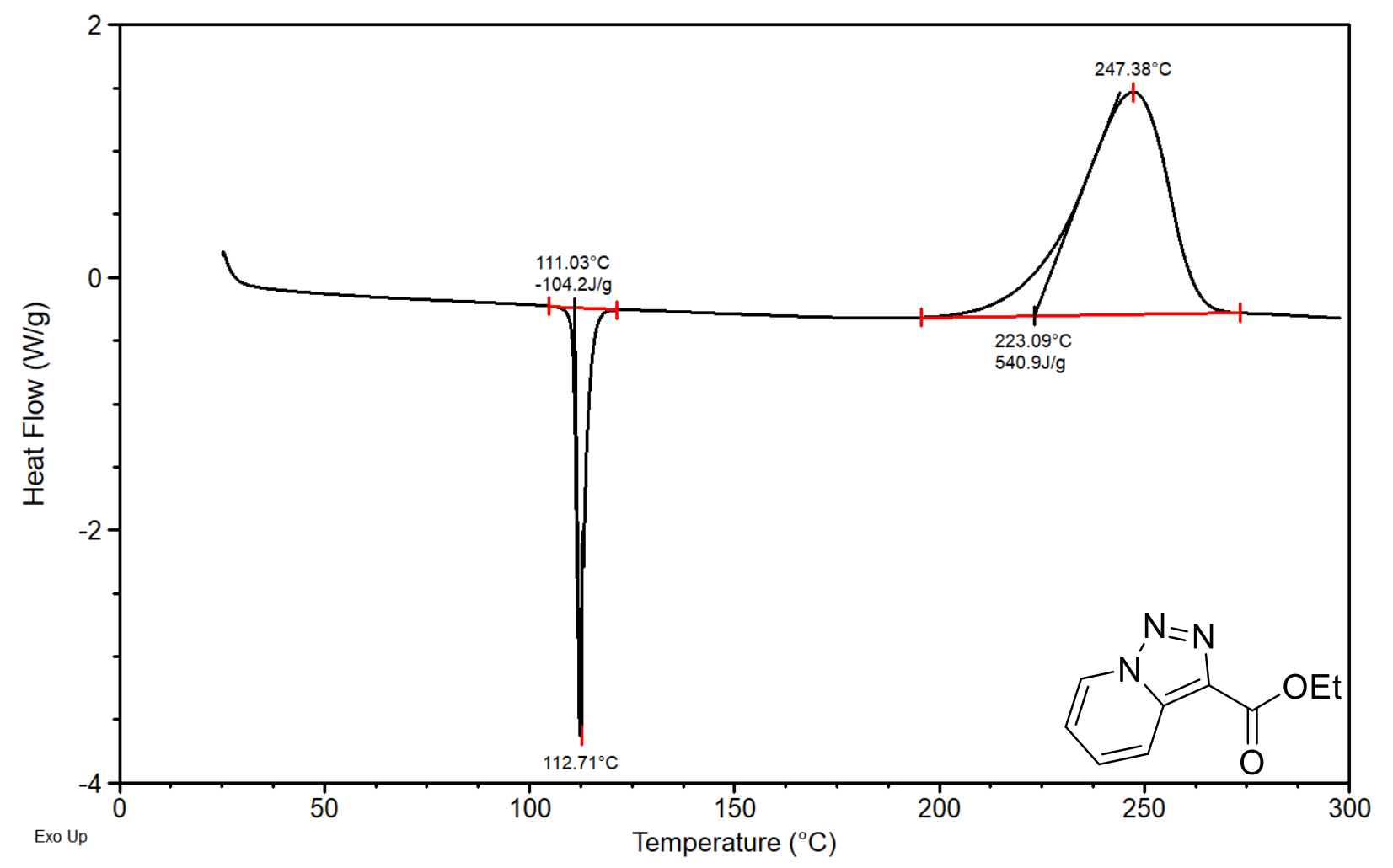


Figure S78. DSC Plot 1 for ethyl diazoacetate (EDA) with $13 \%$ wt. $\mathrm{CH}_{2} \mathrm{Cl}_{2}$

Sample mass $-6.4 \mathrm{mg}$

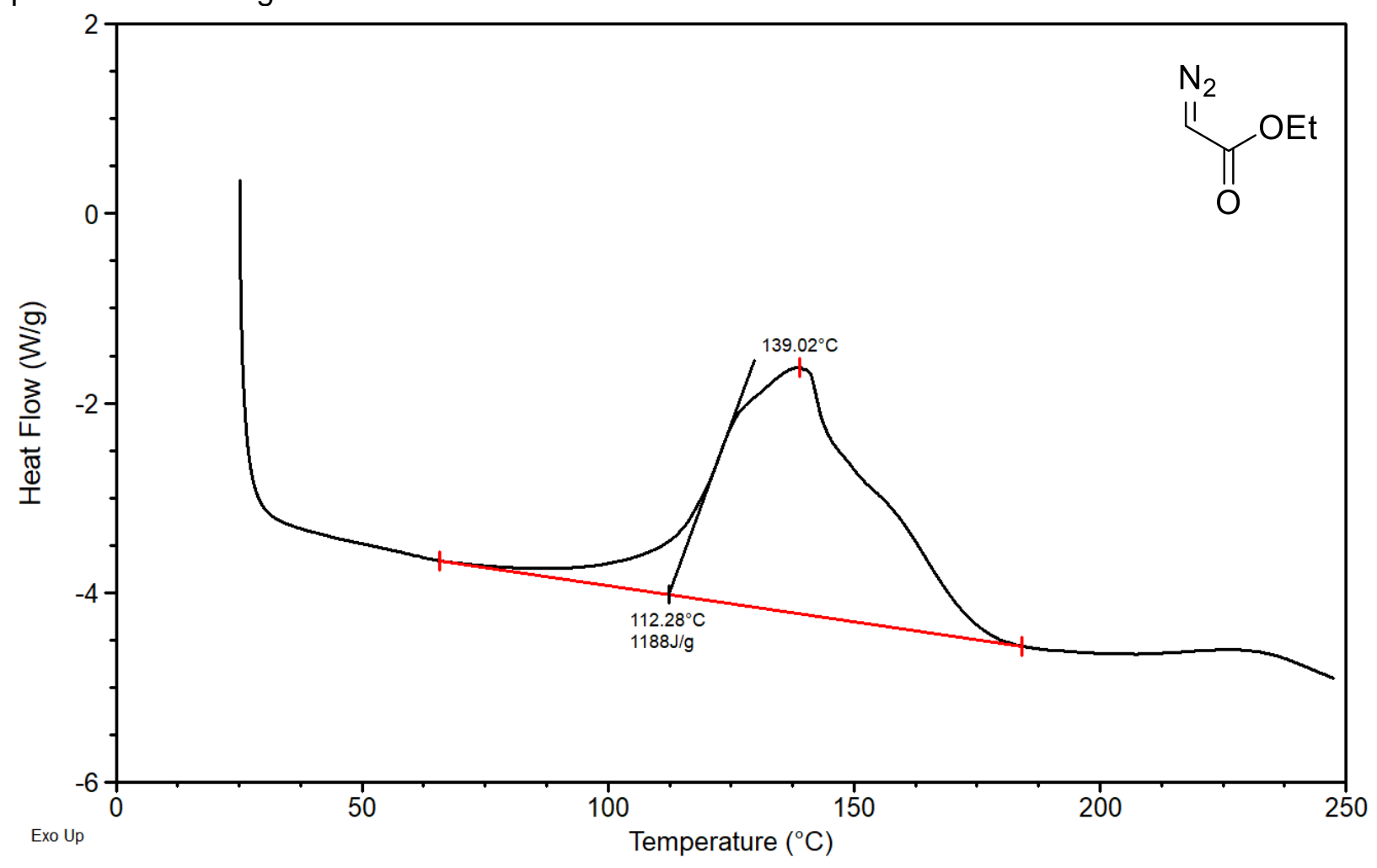

Figure S79. DSC Plot 2 for ethyl diazoacetate (EDA) with $13 \%$ wt. $\mathrm{CH}_{2} \mathrm{Cl}_{2}$

Sample mass $-4.8 \mathrm{mg}$

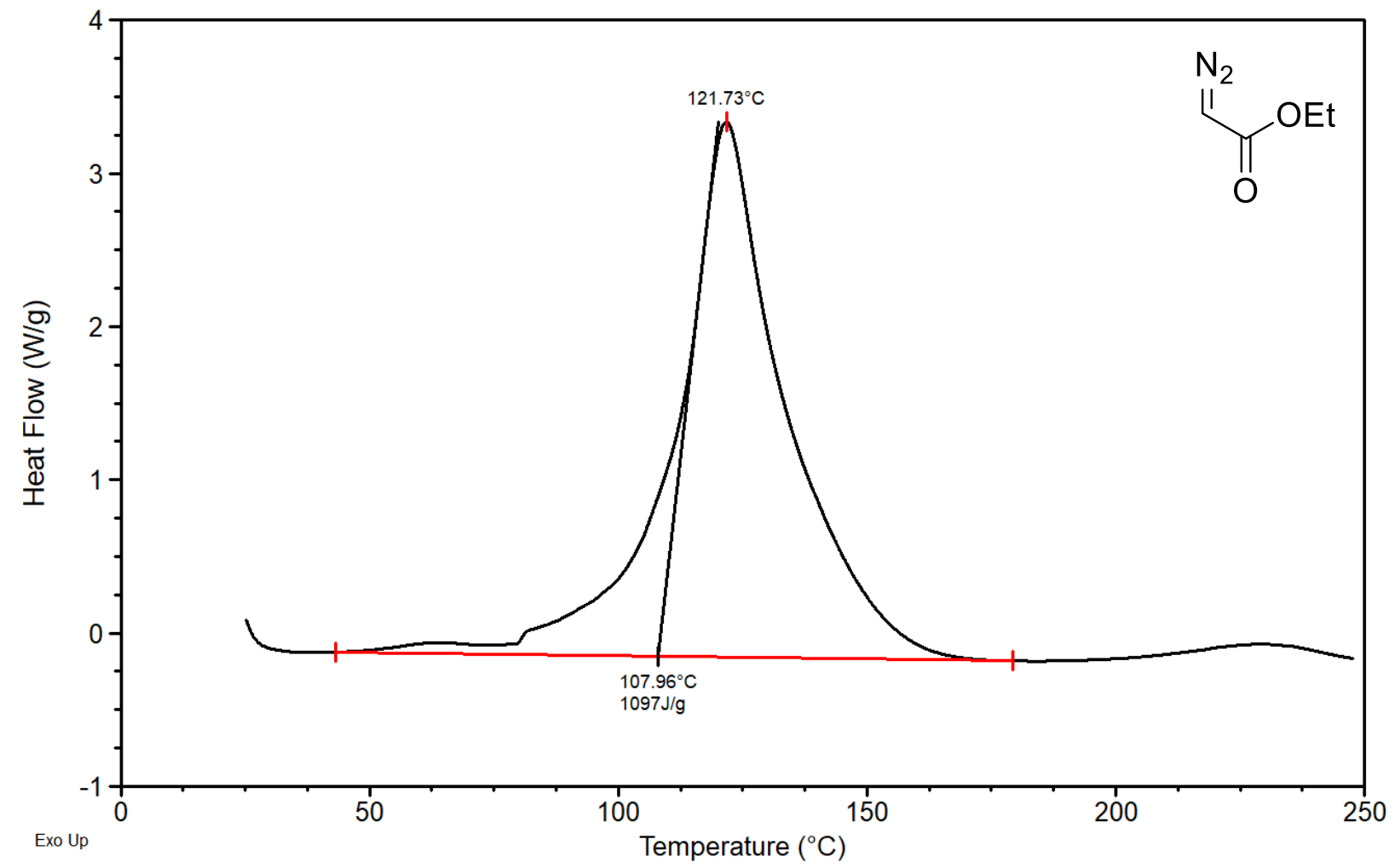


Figure S80. DSC Plot 1 for ethyl (nitro)diazoacetate (64)

Sample mass $-5.7 \mathrm{mg}$

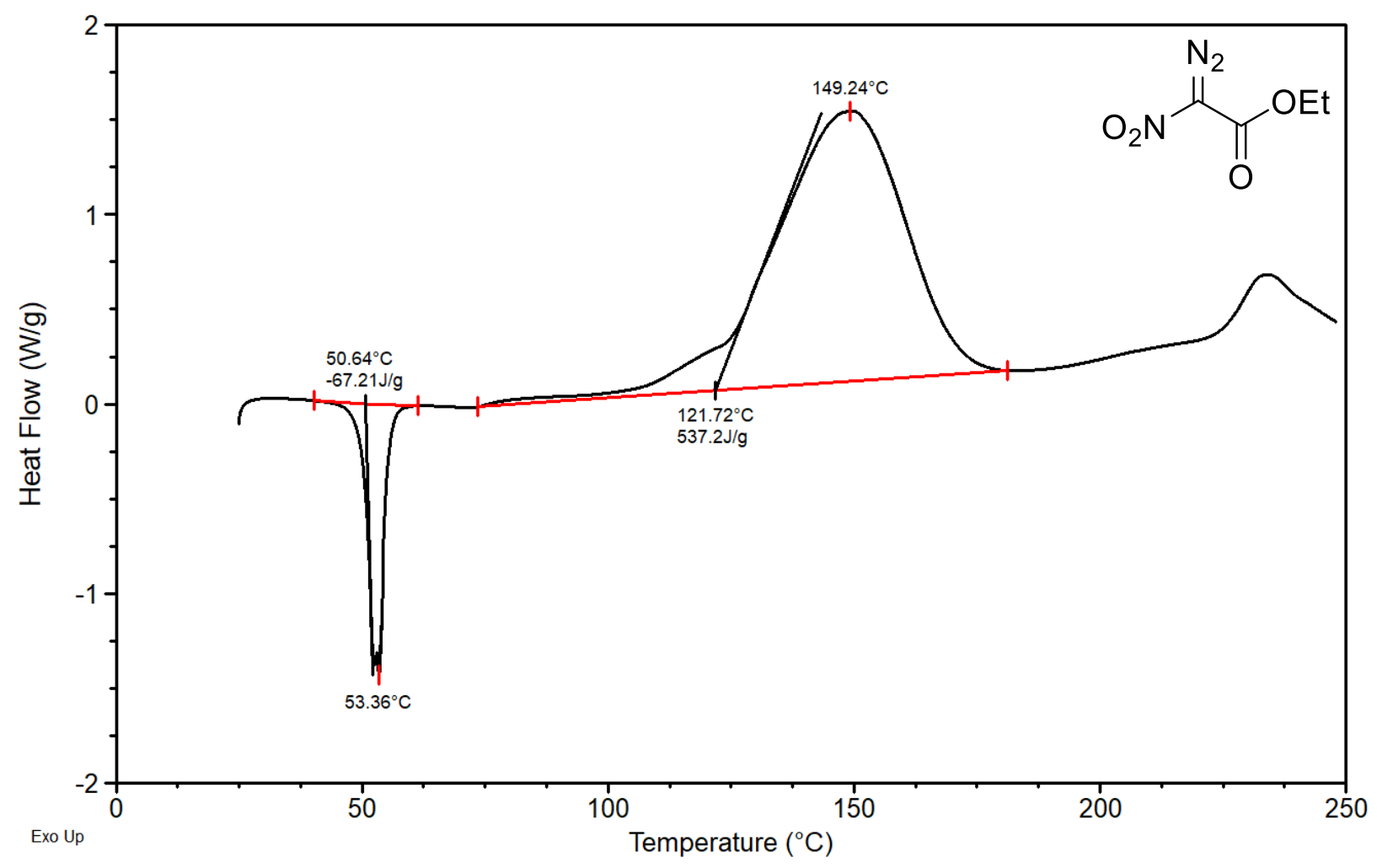

Figure S81. DSC Plot 2 for ethyl (nitro)diazoacetate (64)

Sample mass $-4.2 \mathrm{mg}$

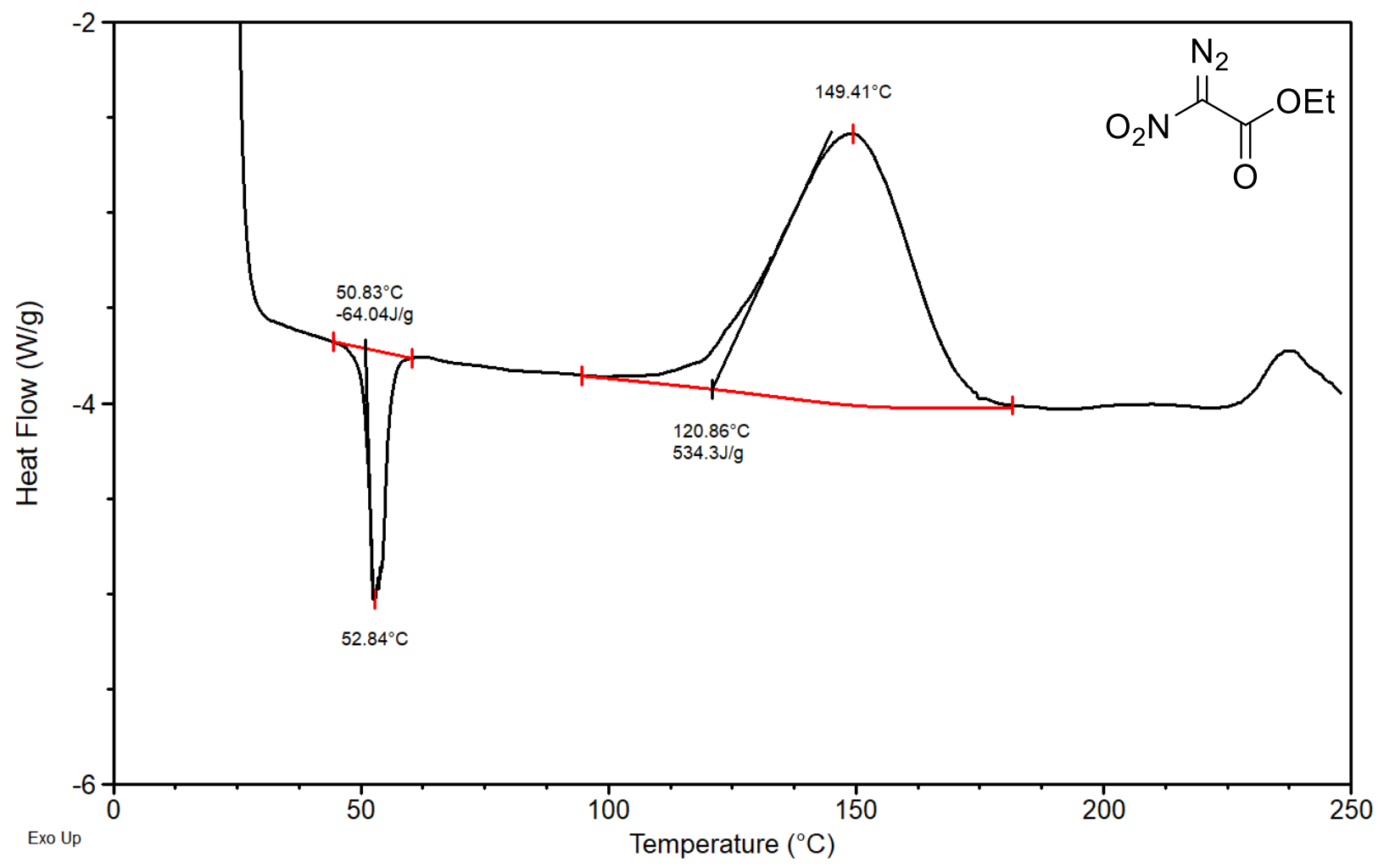


Figure S82. DSC Plot 1 for ethyl (phenylsulfonyl)diazoacetate (65)

Sample mass $-3.7 \mathrm{mg}$

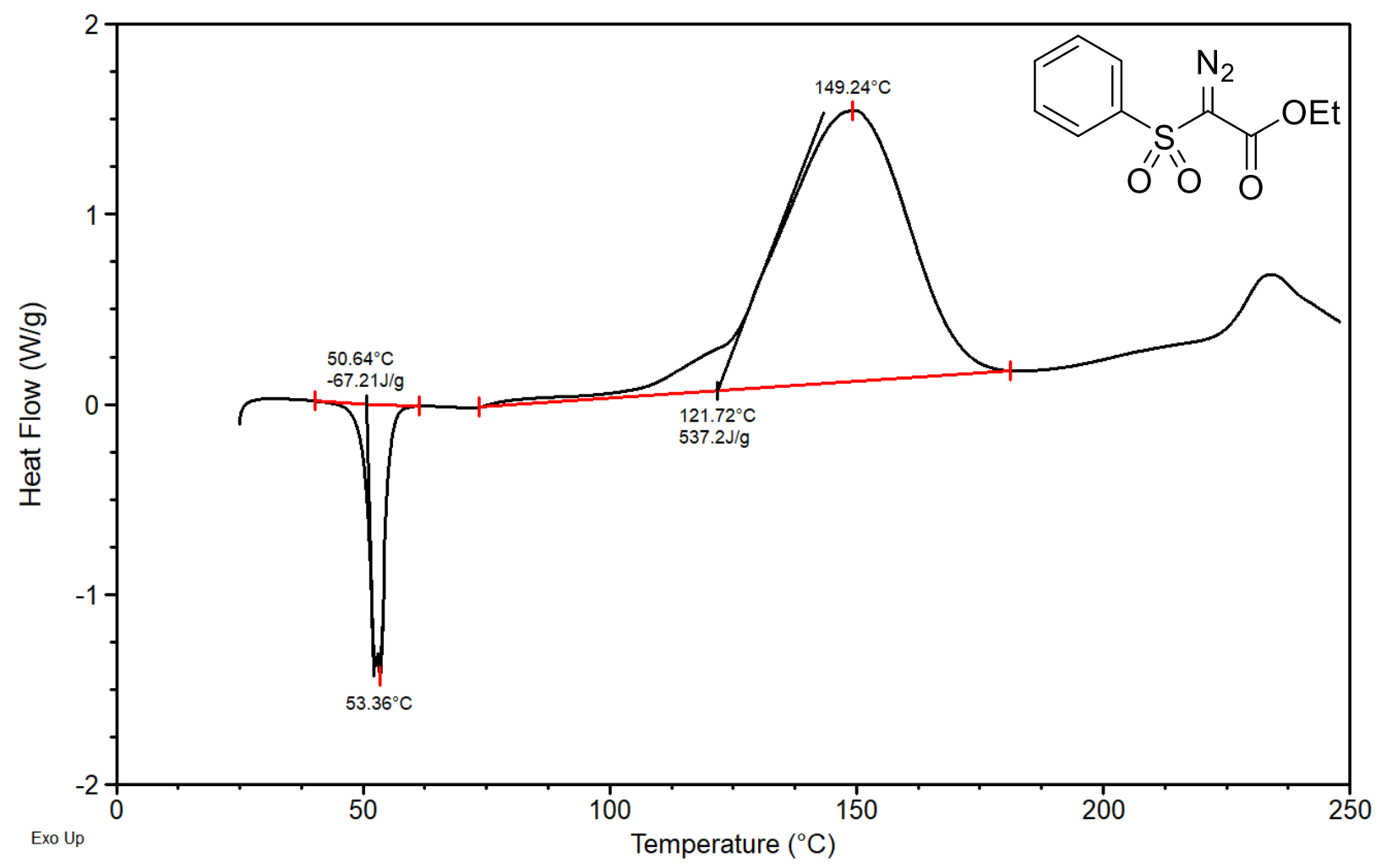

Figure S83. DSC Plot 2 for ethyl (phenylsulfonyl)diazoacetate (65)

Sample mass $-4.0 \mathrm{mg}$

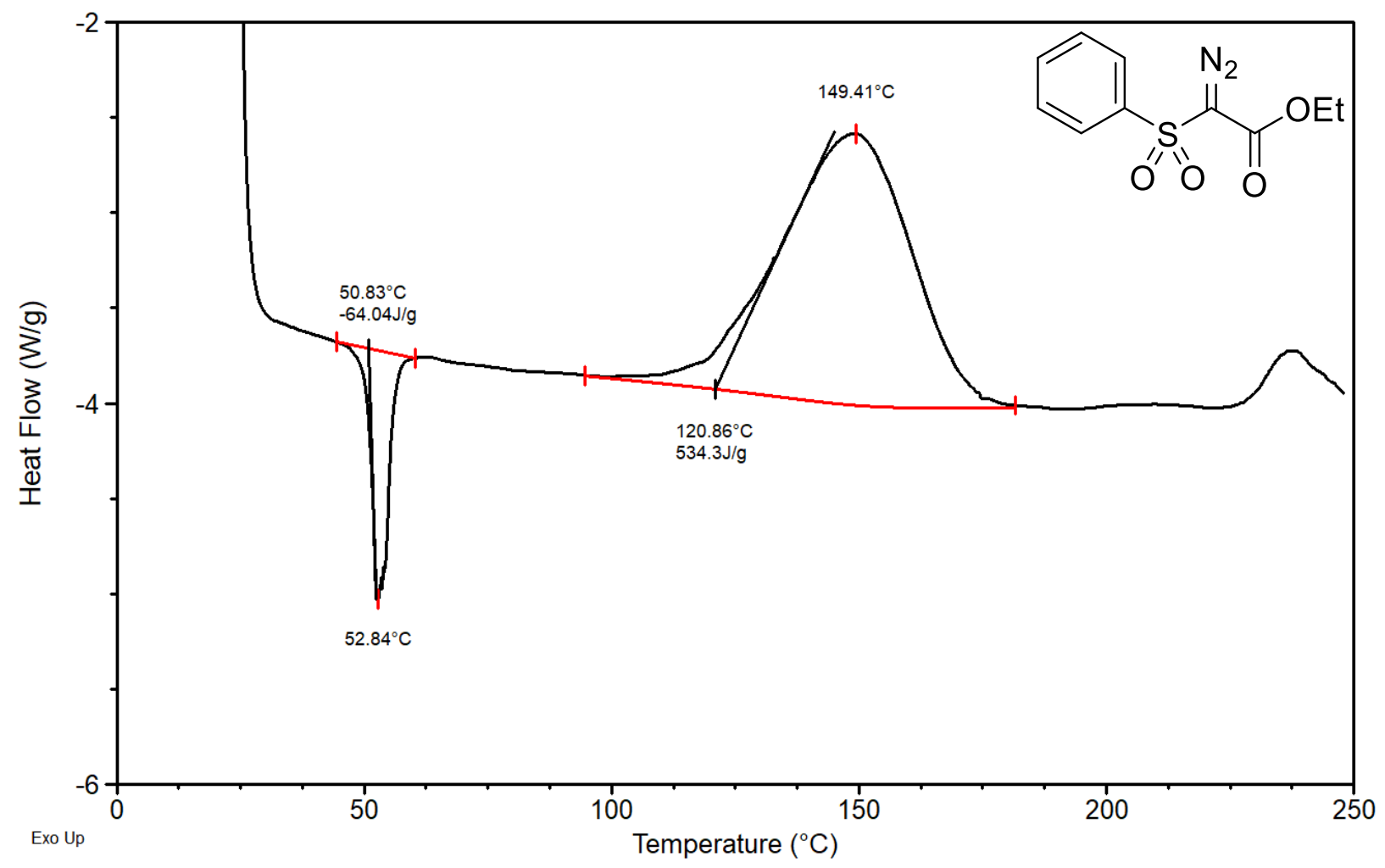


Figure S84. DSC Plot 1 for ethyl 2-diazo-3-oxobutanoate (66)

Sample mass $-4.4 \mathrm{mg}$

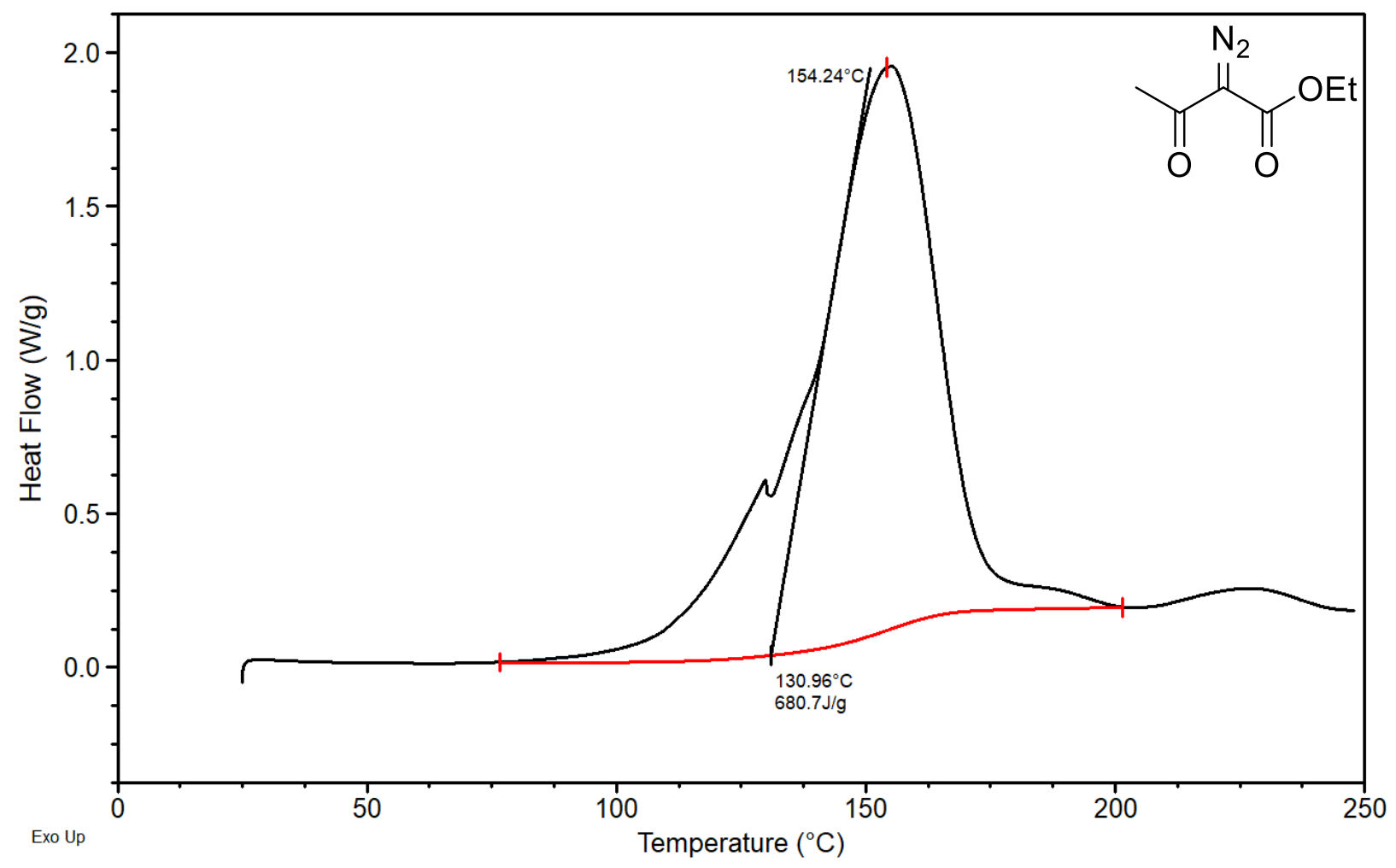

Figure S85. DSC Plot 2 for ethyl 2-diazo-3-oxobutanoate (66)

Sample mass $-5.2 \mathrm{mg}$

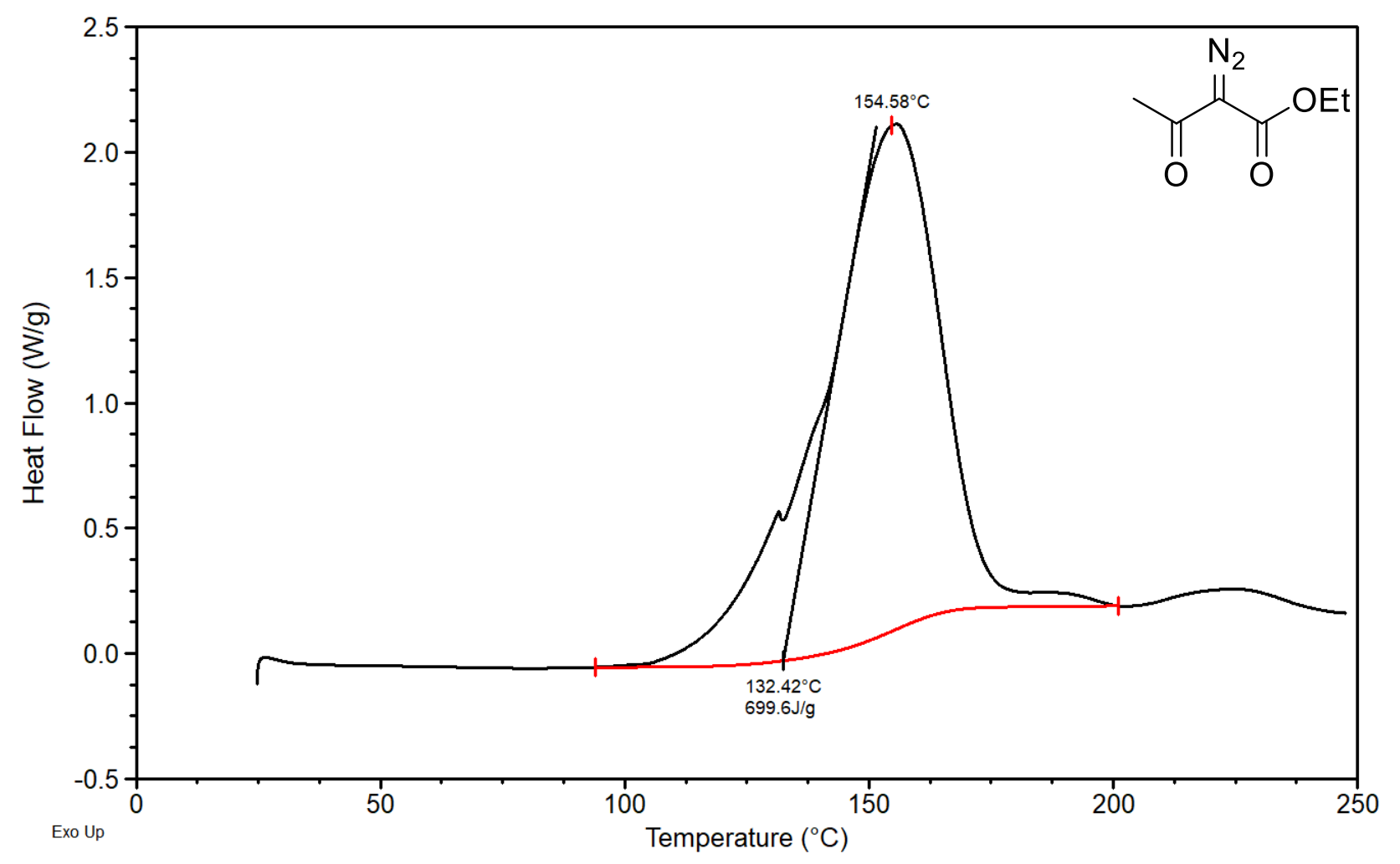


Figure S86. DSC Plot using Method B for ethyl 2-diazo-3-oxobutanoate (66)

Sample mass $-5.0 \mathrm{mg}$

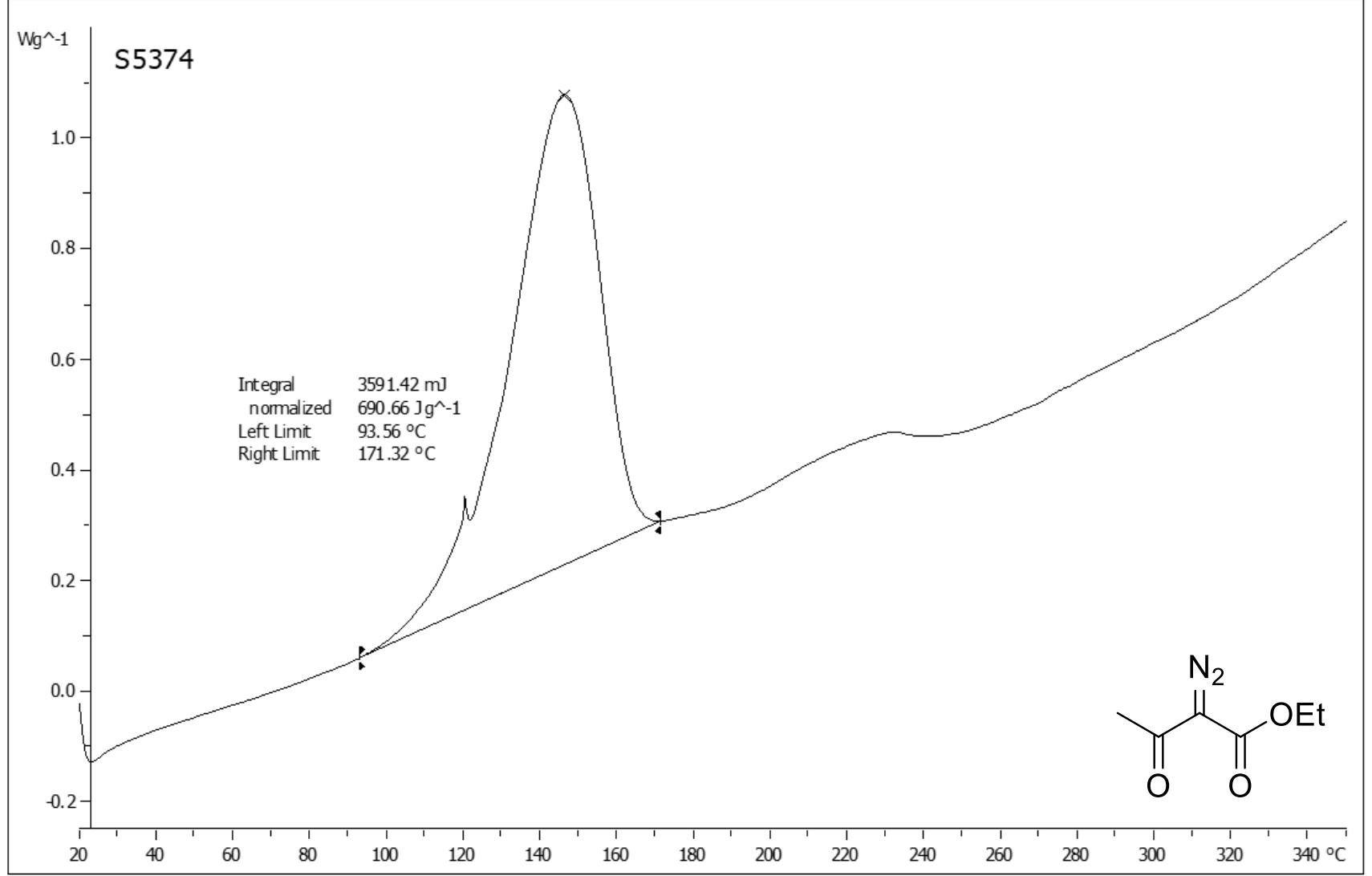


Figure S87. DSC Plot 1 for ethyl 2-diazo-3-oxo-3-(pyrrolidin-1-yl)propanoate (67) Sample mass $-4.5 \mathrm{mg}$

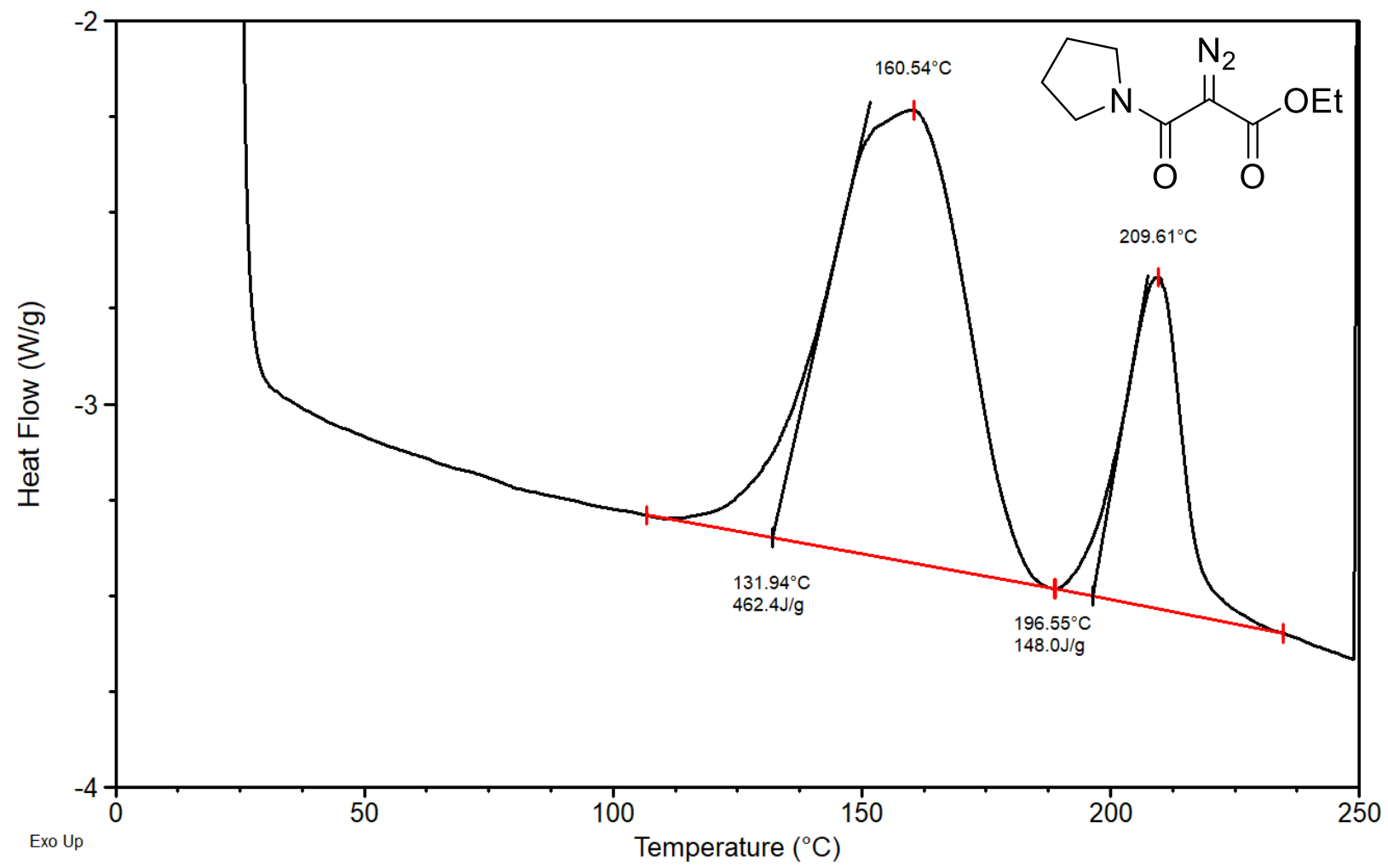

Figure S88. DSC Plot 2 for ethyl 2-diazo-3-oxo-3-(pyrrolidin-1-yl)propanoate (67) Sample mass $-4.1 \mathrm{mg}$

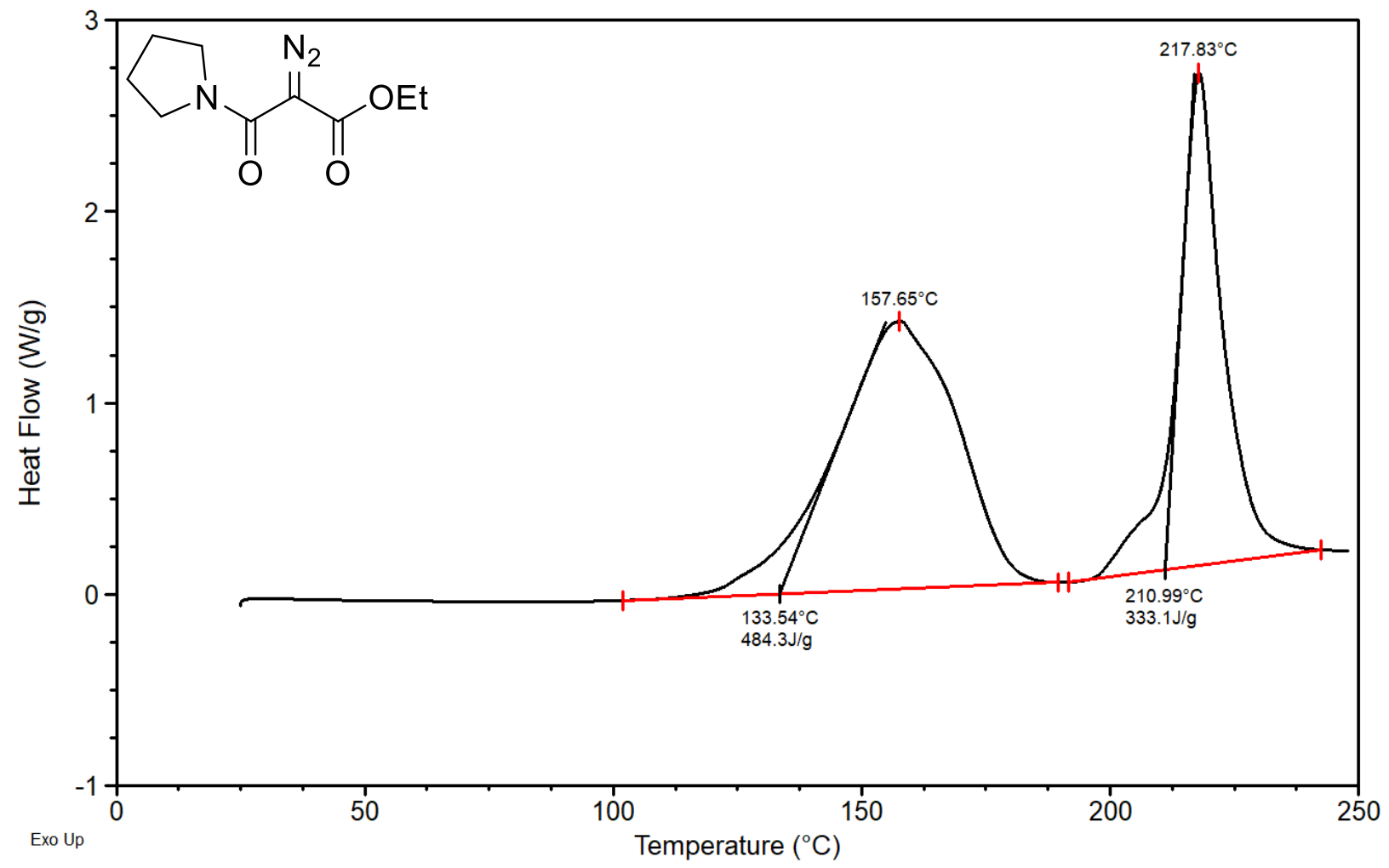


Figure S89. DSC Plot 1 for diethyl 2-diazomalonate (68)

Sample mass $-5.8 \mathrm{mg}$

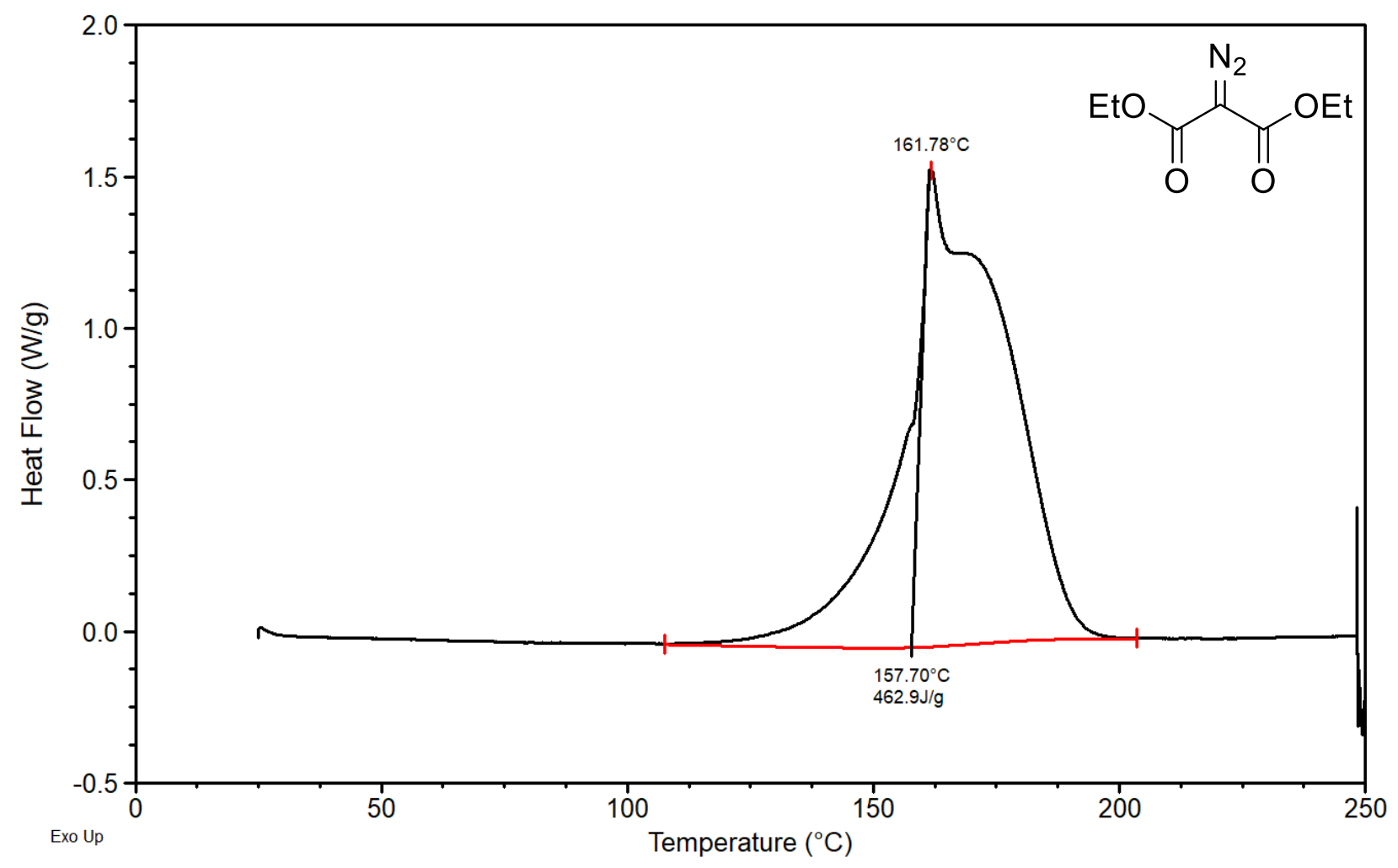

Figure S90. DSC Plot 2 for diethyl 2-diazomalonate (68)

Sample mass $-4.7 \mathrm{mg}$

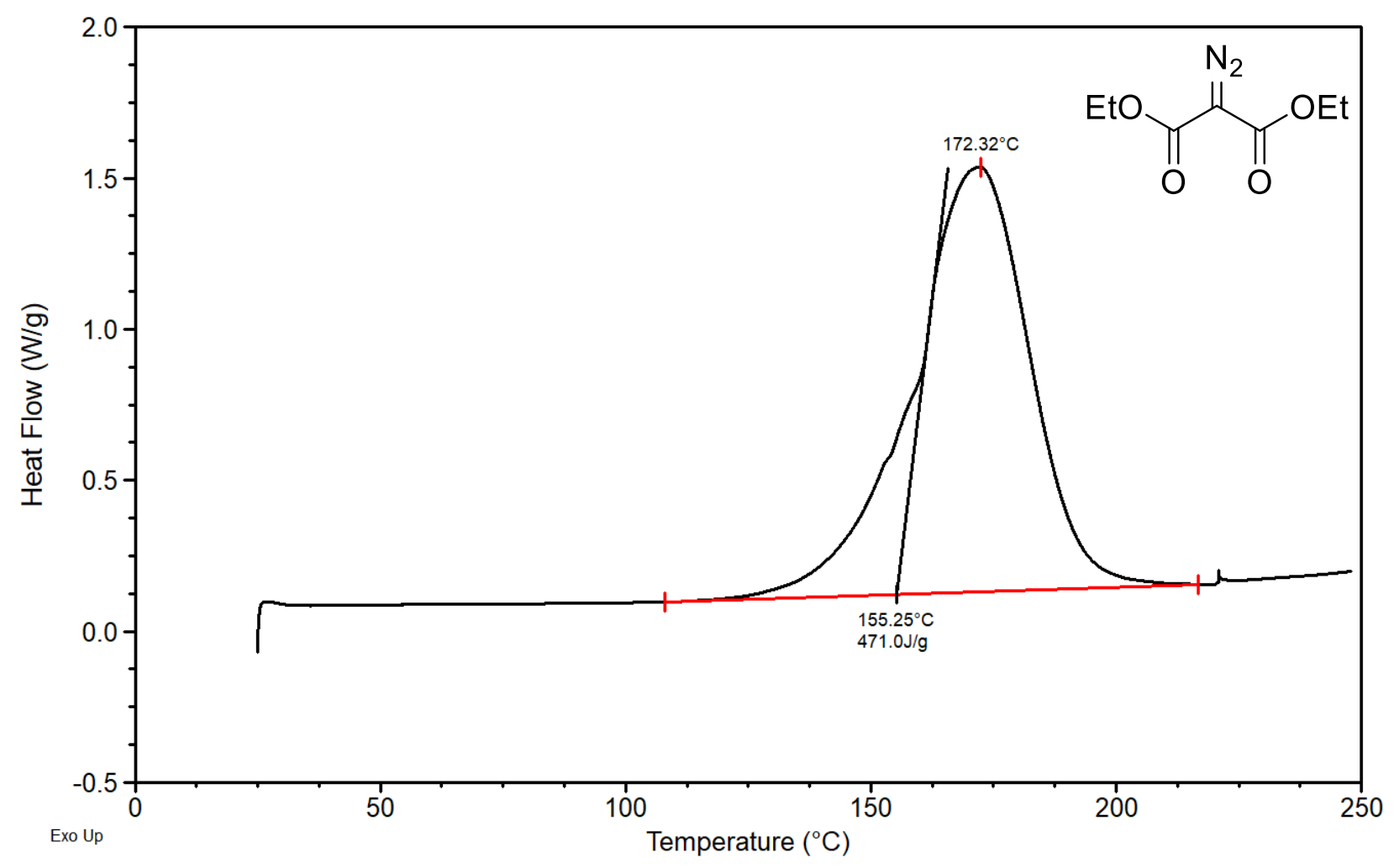


Figure S91. DSC Plot 1 for ethyl (diethoxyphosphoryl)diazoacetate (69)

Sample mass $-6.1 \mathrm{mg}$

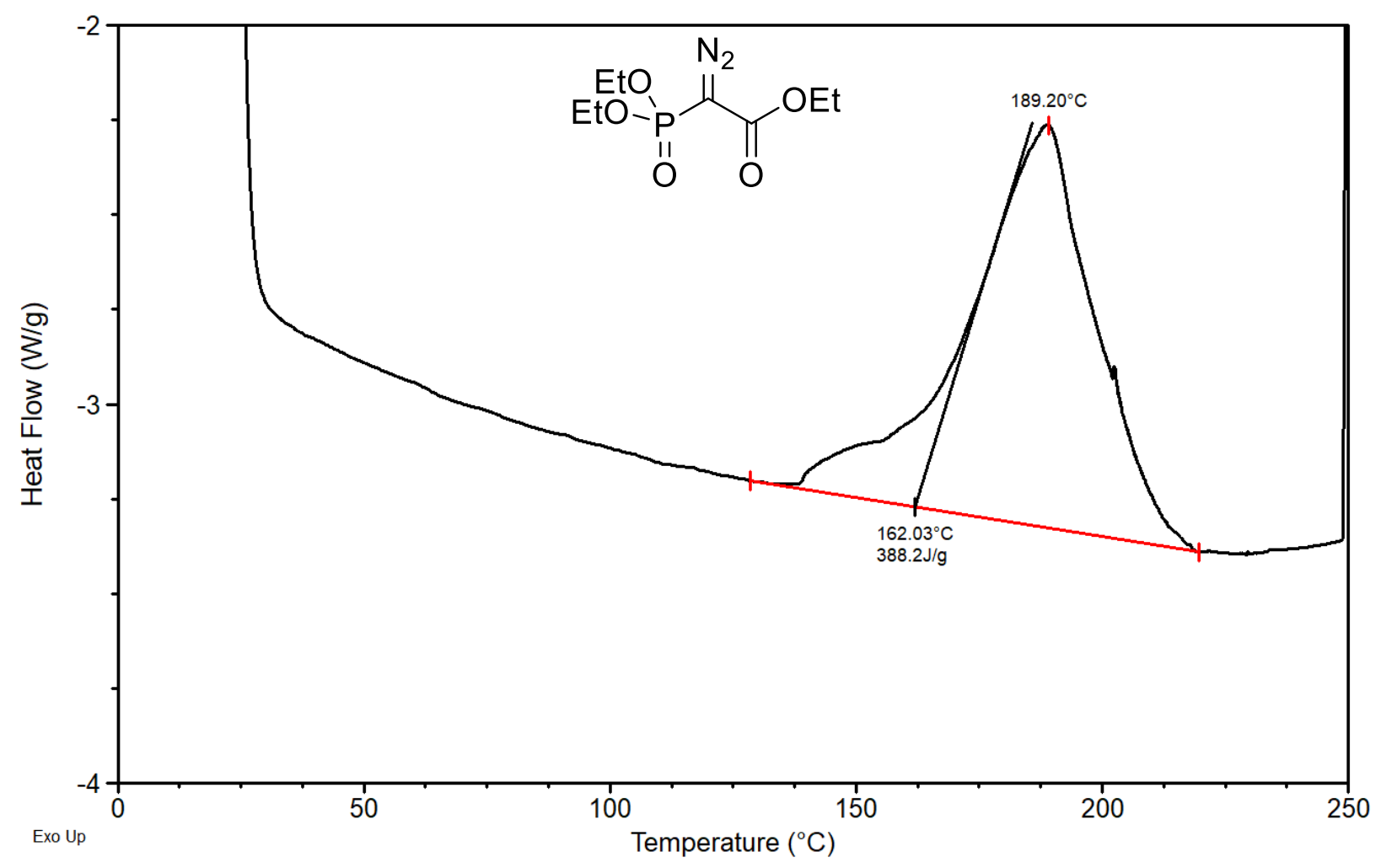

Figure S92. DSC Plot 2 for ethyl (diethoxyphosphoryl)diazoacetate (69)

Sample mass $-4.7 \mathrm{mg}$

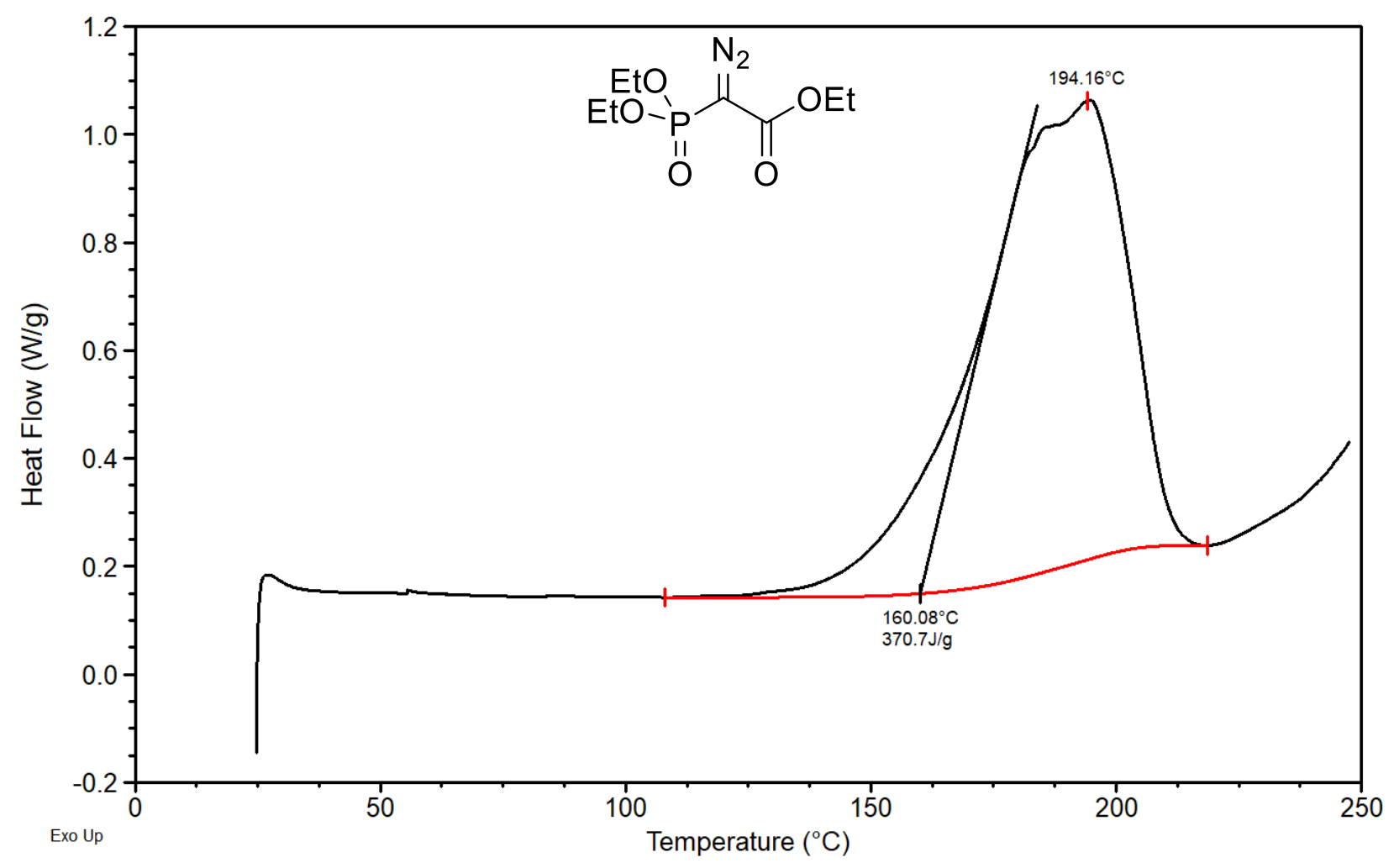


Figure S93. DSC Plot 1 for cyanodiazo-N,N-diethylacetamide (70)

Sample mass $-4.9 \mathrm{mg}$

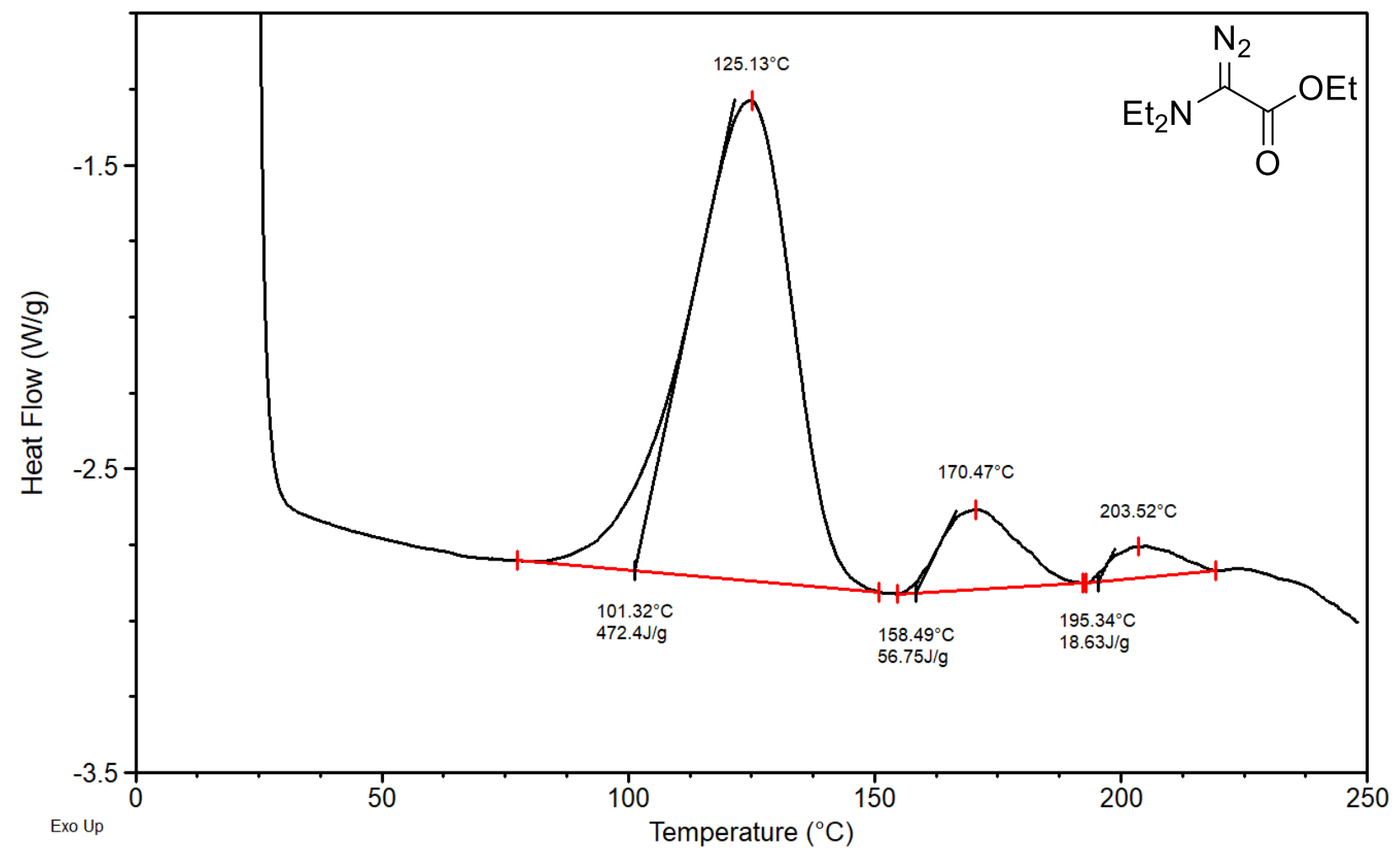

Figure S94. DSC Plot 2 for cyanodiazo-N,N-diethylacetamide (70)

Sample mass $-5.7 \mathrm{mg}$

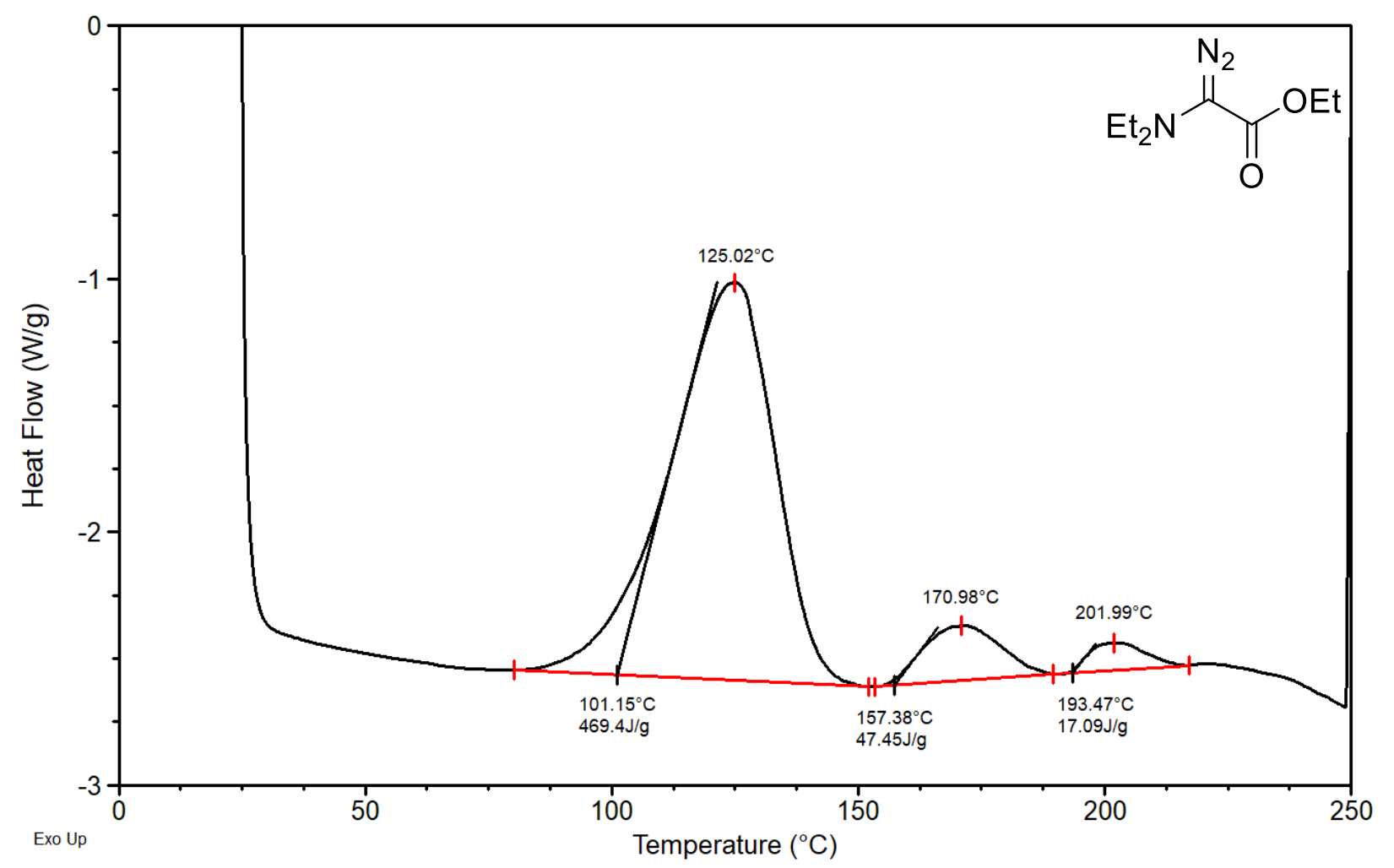


Figure S95. DSC Plot 1 for benzyl (cyano)diazoacetate (71)

Sample mass $-5.1 \mathrm{mg}$

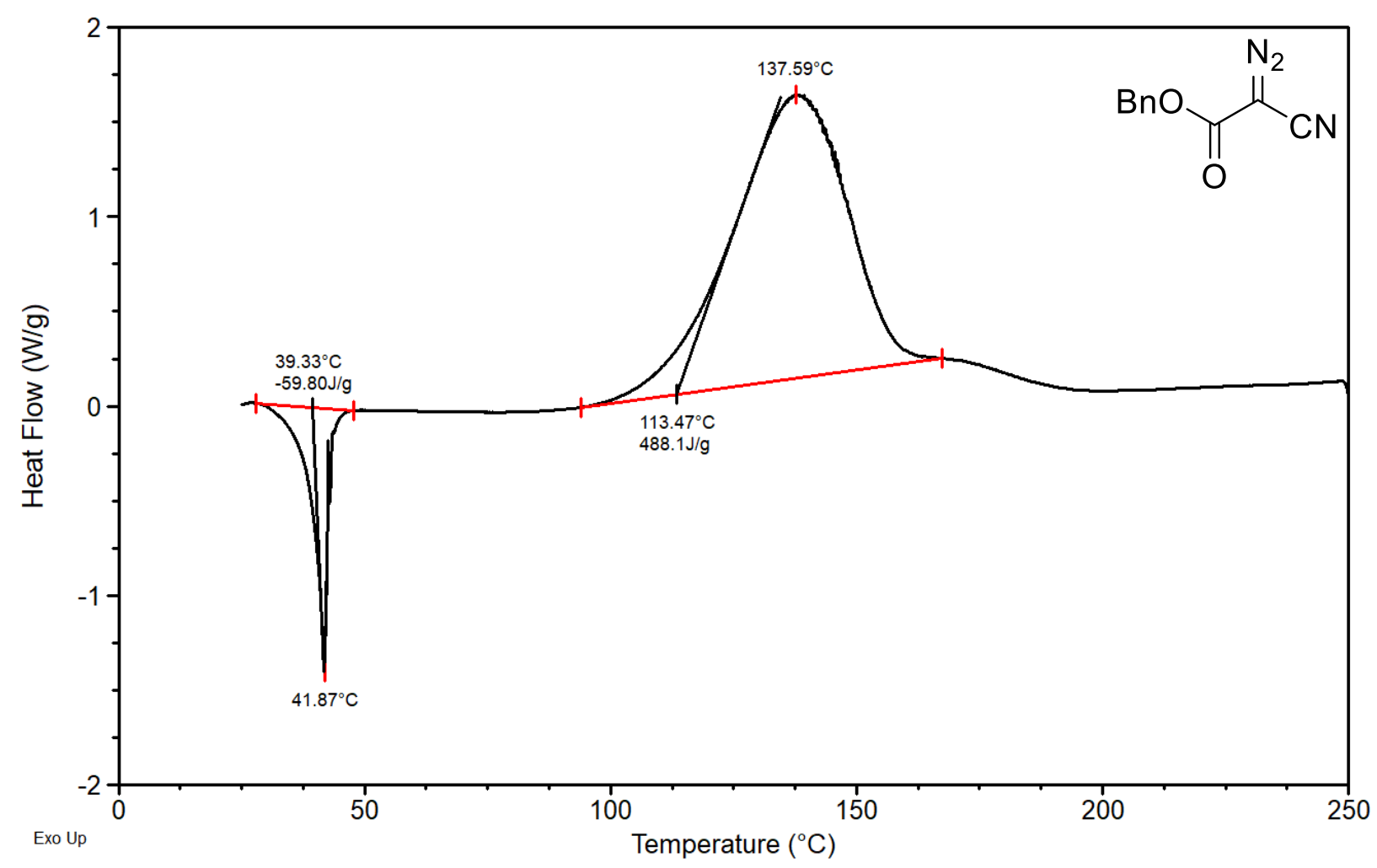

Figure S96 DSC Plot 2 for benzyl (cyano)diazoacetate (71)

Sample mass $-3.6 \mathrm{mg}$

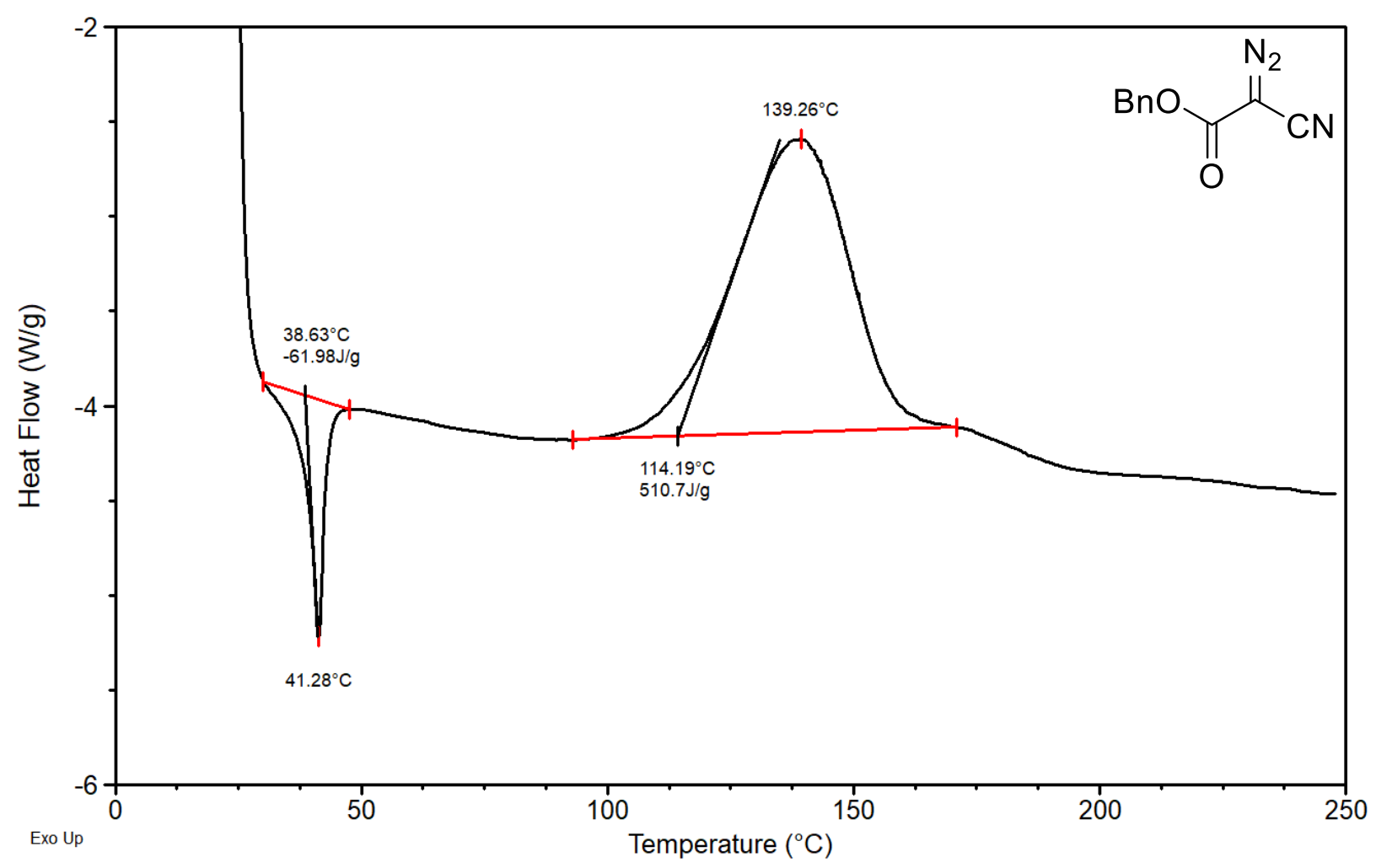


S. P. Green, K. M. Wheelhouse, A. D. Payne, J. P. Hallett, P. W. Miller and J. A. Bull

Figure S97. DSC Plot 1 for tert-butyl 4-(cyanodiazoacetyl)piperazine-1-carboxylate (72) Sample mass $-1.7 \mathrm{mg}$

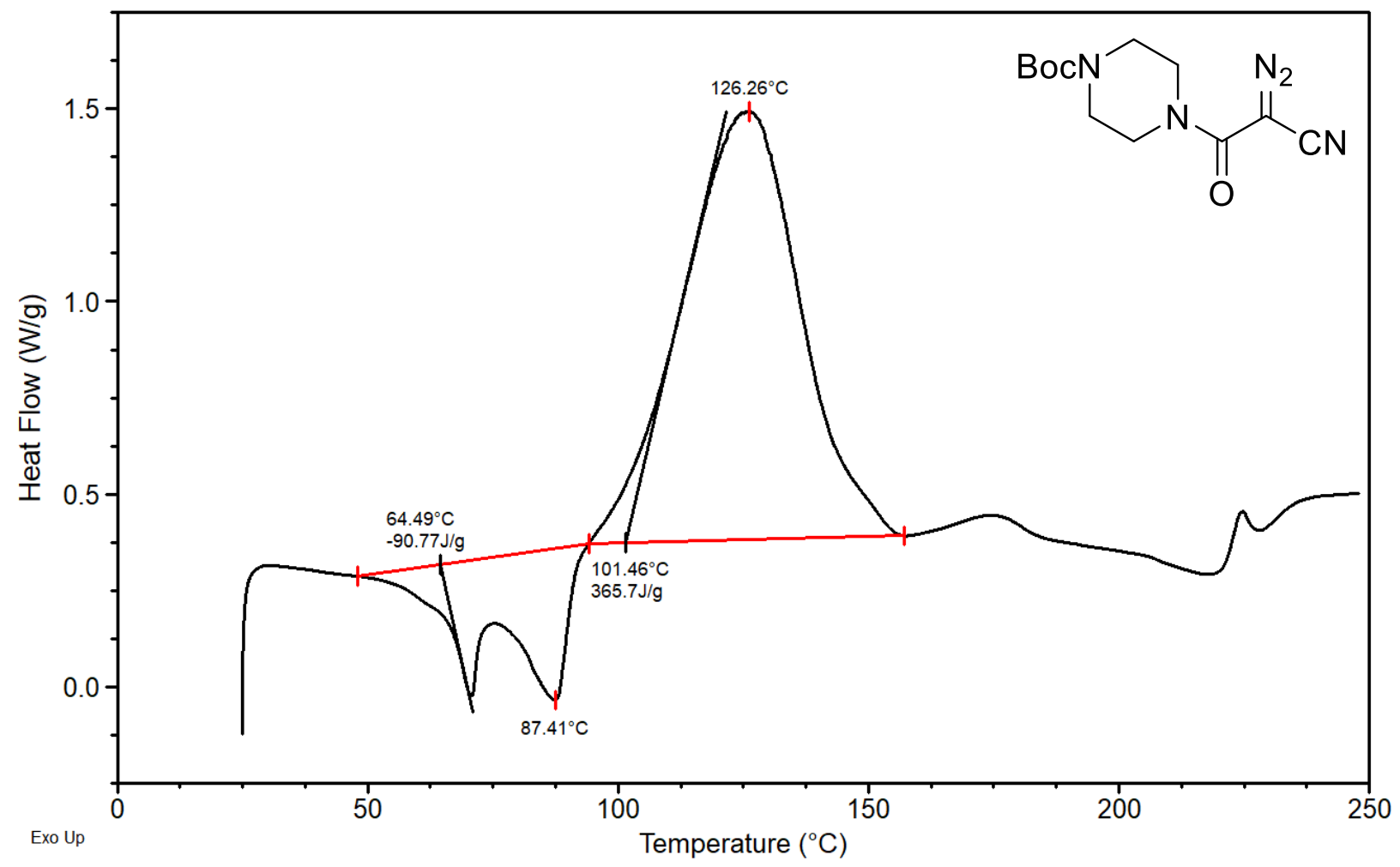

Figure S98. DSC Plot 2 for tert-butyl 4-(cyanodiazoacetyl)piperazine-1-carboxylate (72) Sample mass $-5.0 \mathrm{mg}$

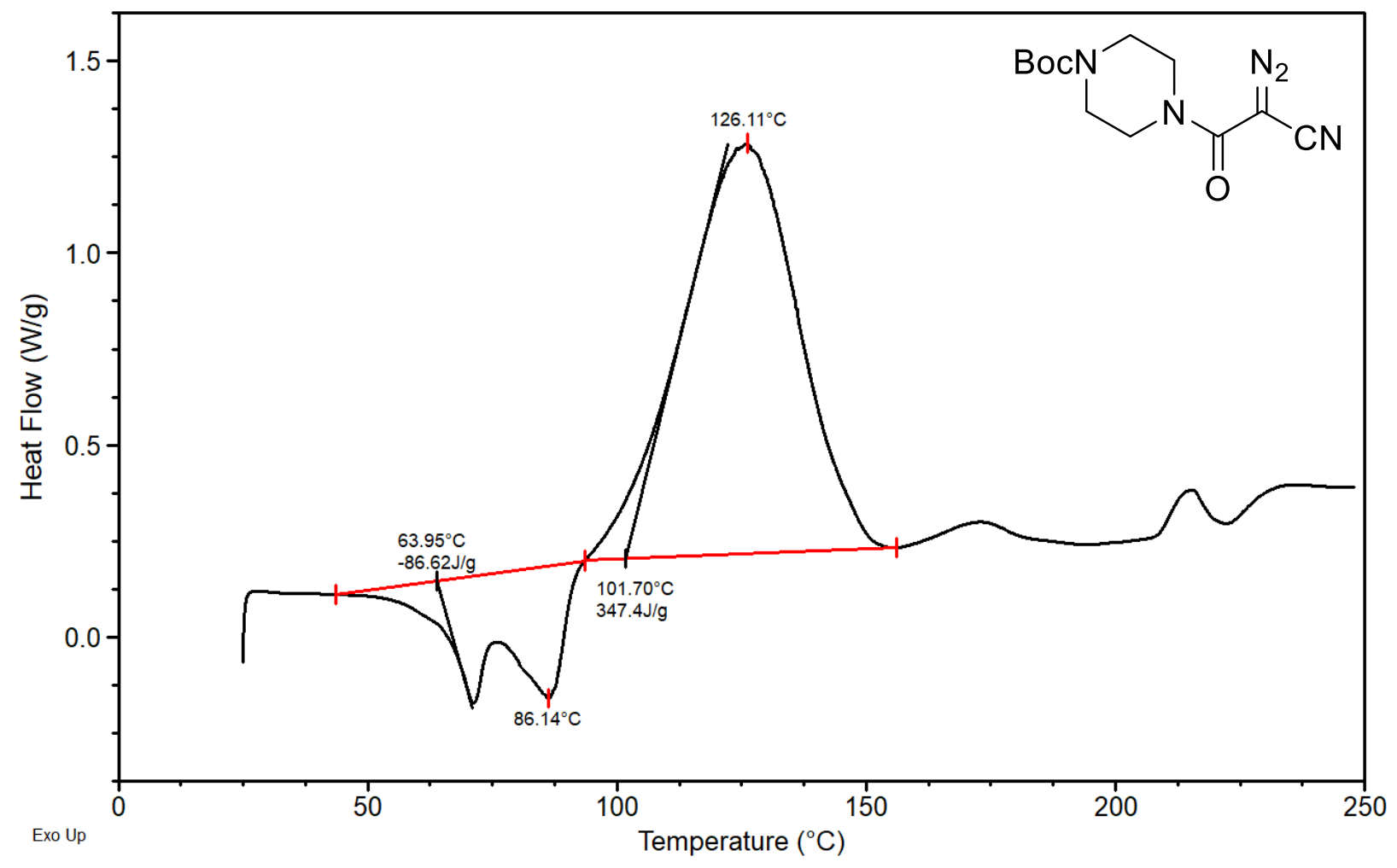


Figure S99. DSC Plot 1 for (1-diazo-2,2,2-trifluoroethyl)benzene (73)

Sample mass $-6.0 \mathrm{mg}$

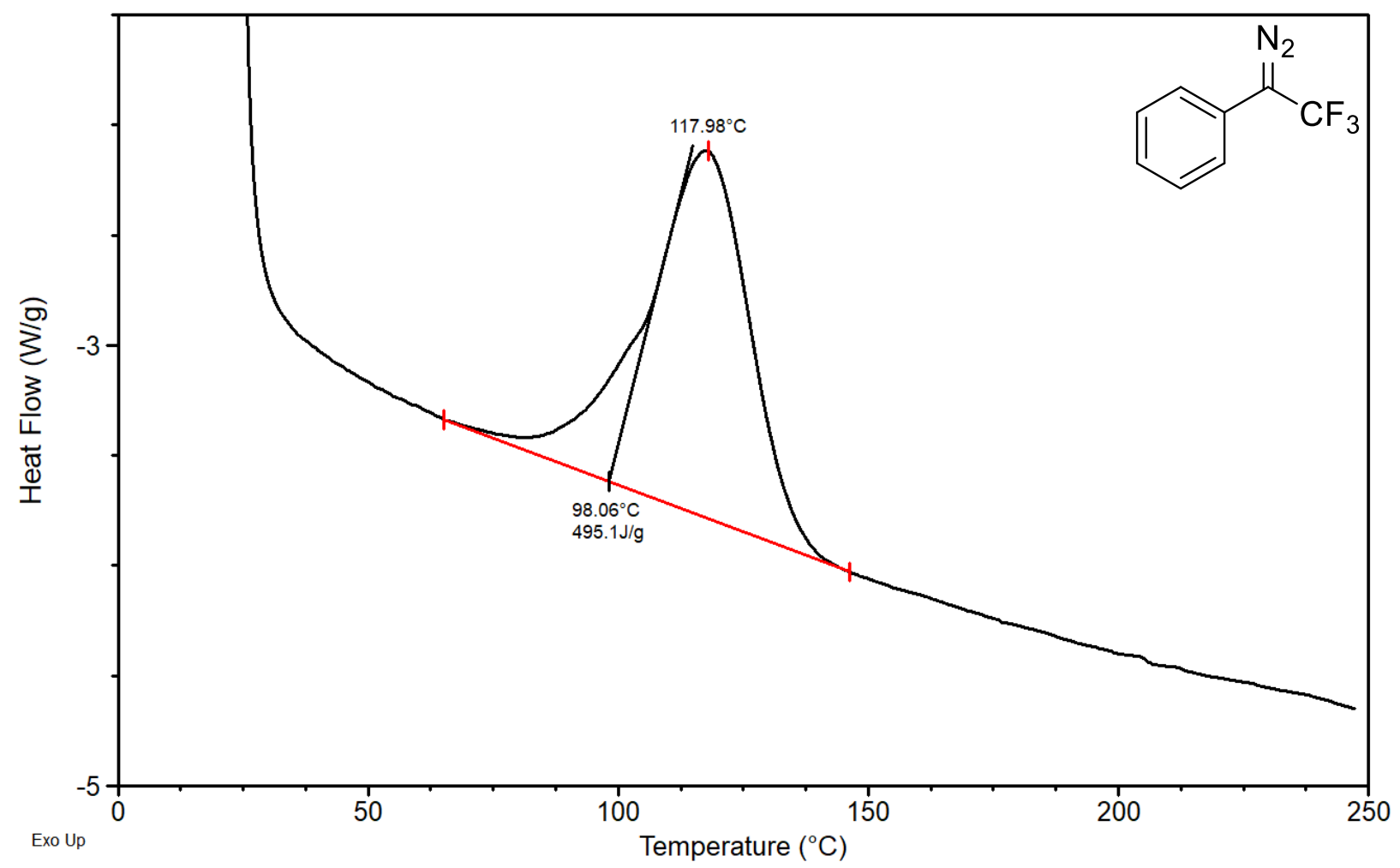

Figure S100. DSC Plot 2 for (1-diazo-2,2,2-trifluoroethyl)benzene (73)

Sample mass $-5.8 \mathrm{mg}$

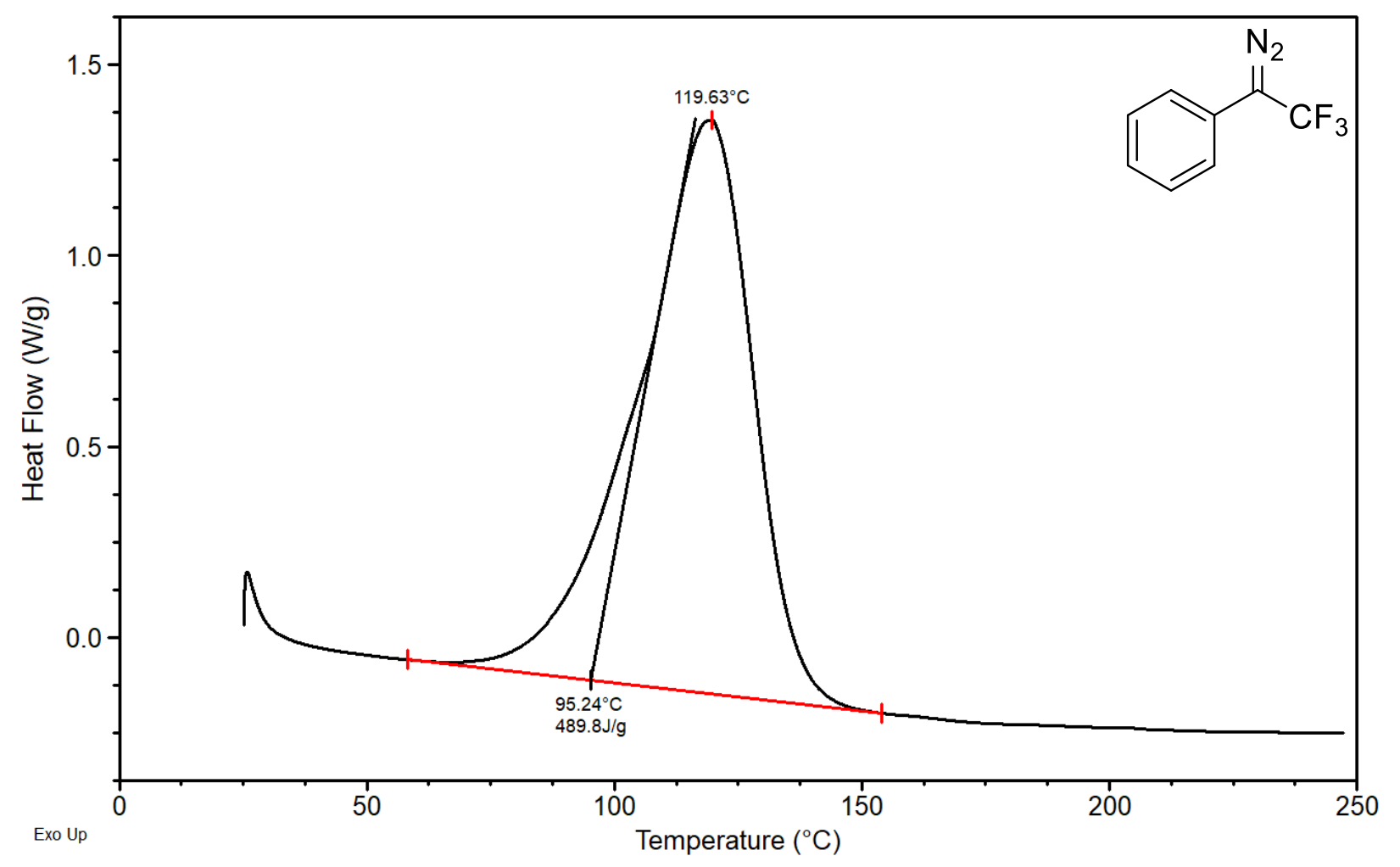


Figure S101. DSC Plot 1 for 1-bromo-4-(1-diazo-2,2,2-trifluoroethyl)benzene (74) Sample mass $-4.2 \mathrm{mg}$

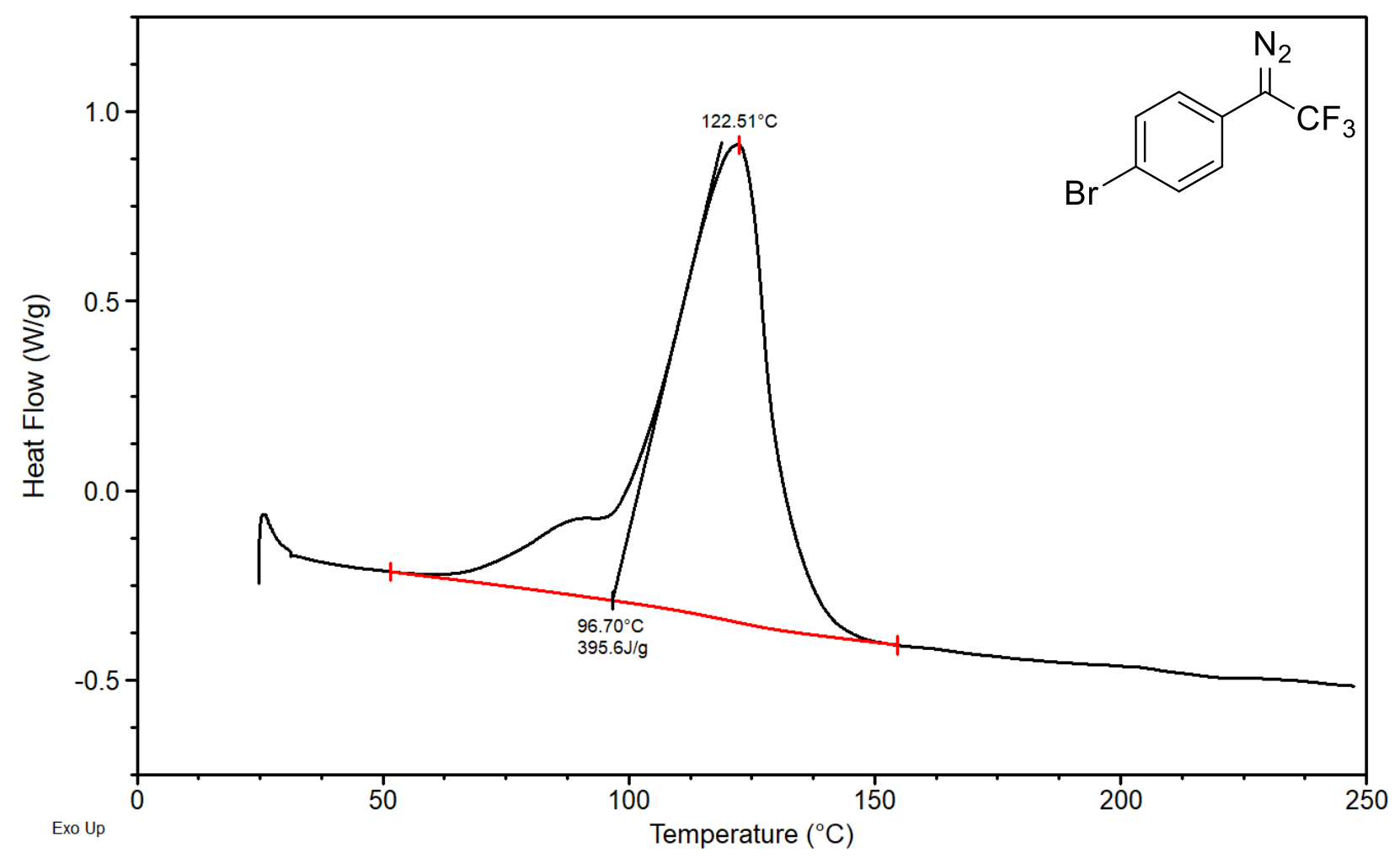

Figure S102. DSC Plot 2 for 1-bromo-4-(1-diazo-2,2,2-trifluoroethyl)benzene (74) Sample mass $-5.3 \mathrm{mg}$

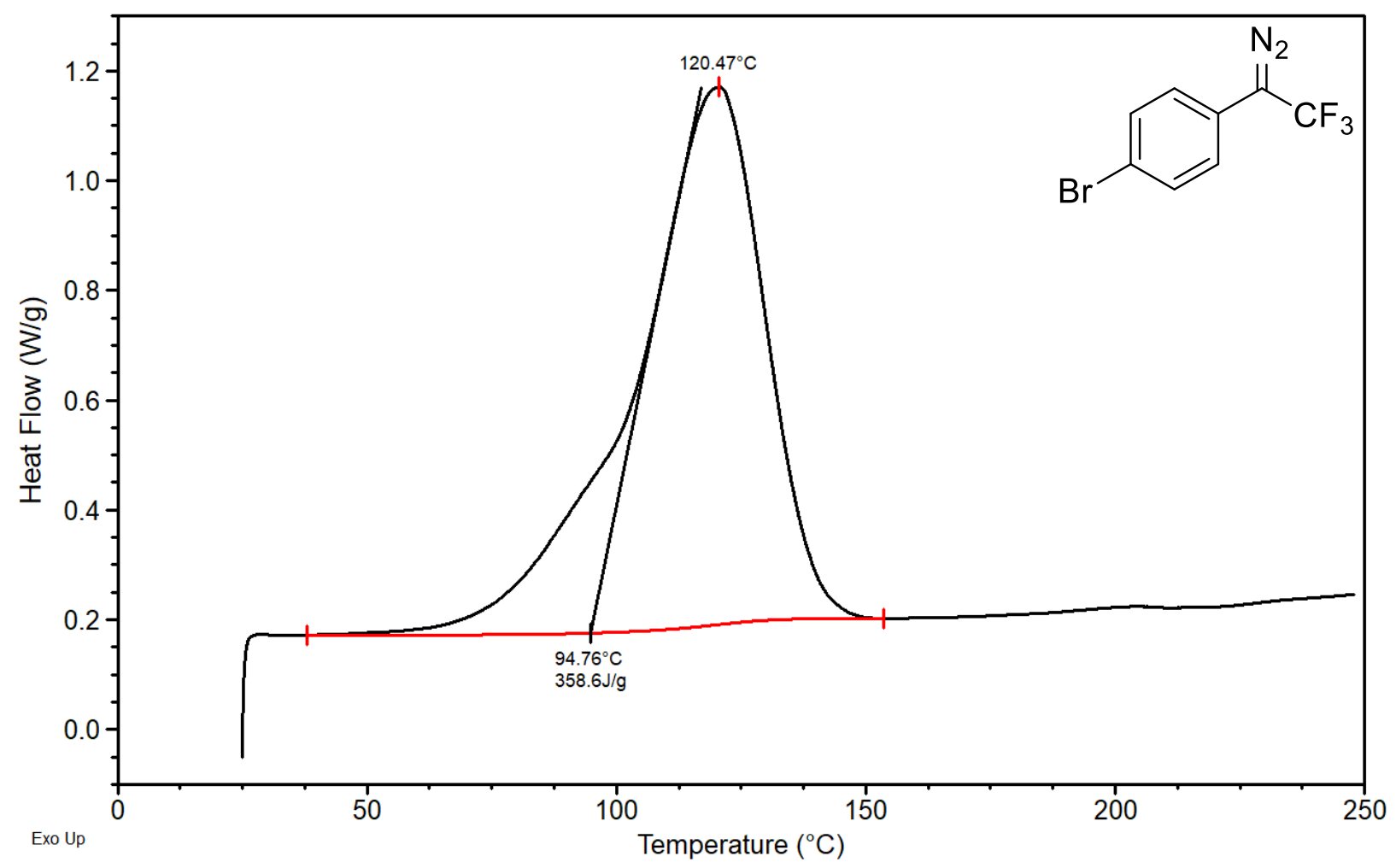


Figure S103. Abnormalities observed in DSC plots of Diazo Compounds
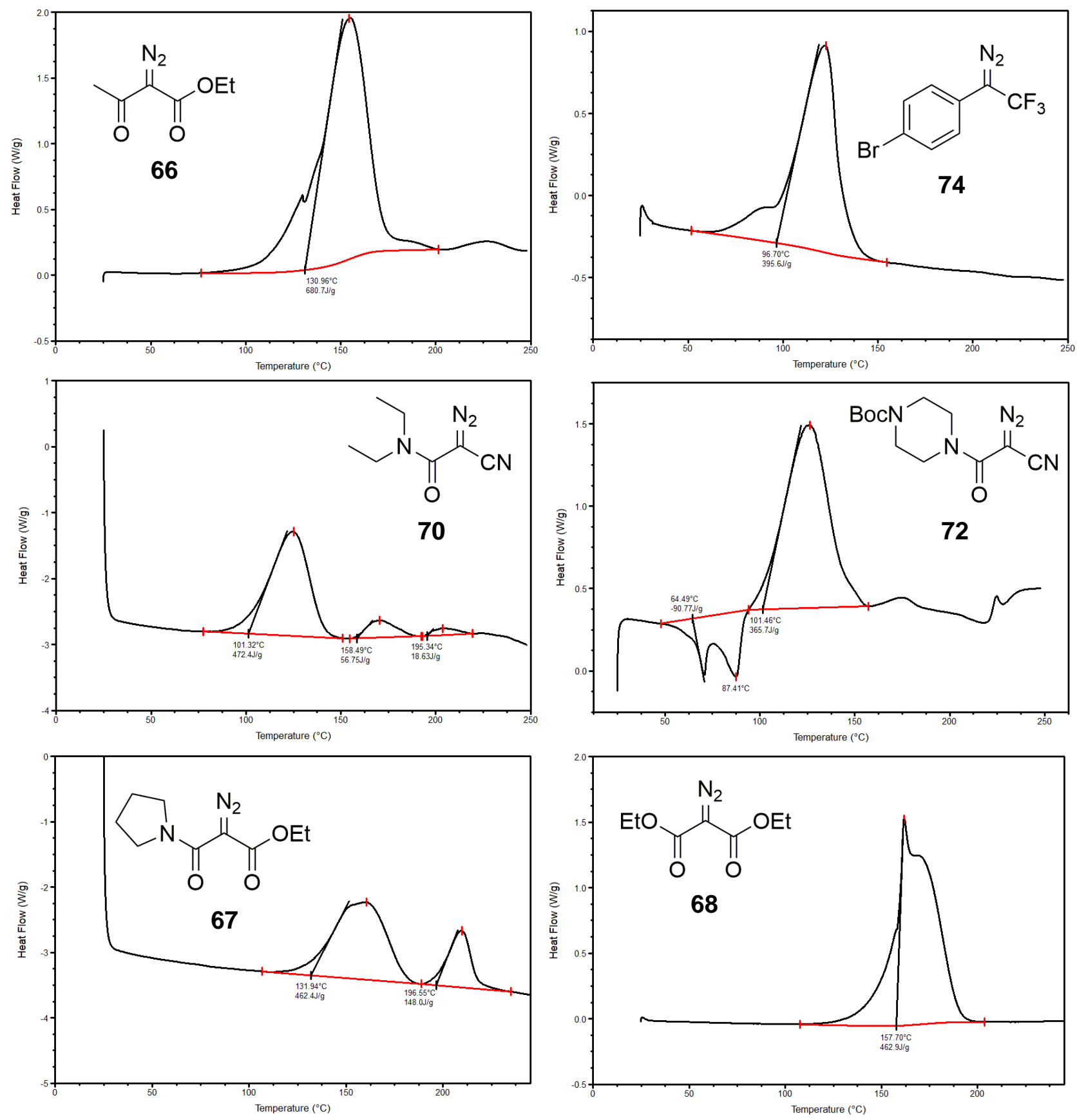

In several cases, abnormalities in the DSC plot are observed which affect thermal stability and enthalpy results, and indicate the measured transition was not only $\mathrm{N}_{2}$ dissociation (see Figure $\mathrm{S} 1$ for full details). Ester-ketone 66 and both aryl-- $\mathrm{CF}_{3}$ diazo compounds 73 and 74 had a small endotherm within the decomposition. As $\mathbf{7 3}$ and $\mathbf{7 4}$ were observed to be somewhat volatile, this is potentially endothermic vaporization. Many compounds experienced a minor second exotherm of around $5 \mathrm{~kJ} \mathrm{~g}^{-1}$ but the most notable were unsubstituted pyridyls 60 and 61 and amides 67 and 70 , with secondary exotherms of -32 , $-44,-54$ and $-9 \mathrm{~kJ} \mathrm{~mol}^{-1}$ respectively. Complex cyanodiazo-amide 72 has overlapping melting and decomposition, giving a complex DSC plot. Diazomalonate 68 has an unusual peak shape suggesting an additional endo- or exothermic transition during decomposition, which could be due to vaporization or $\mathrm{CO}_{2}$ off-gassing. As a result, the $T_{\text {onset }}$ is increased and $\Delta H_{\mathrm{D}}$ measurement is the sum of enthalpies for decomposition and the additional transitions. 


\section{Table S2. Yoshida IS and EP Correlations for Literature Diazo Transfer Reagent Data}

The Yoshida correlation was applied to the diazo transfer reagent data obtained from existing literature (Table $\mathbf{2}$ in the manuscript). The correlation prediction is compared to literature reports of impact sensitivity (IS) and explosivity in the table below. Values $>0$ (in red) indicate a positive prediction of the property; values $<0$ indicate a negative prediction.

\begin{tabular}{|c|c|c|c|c|c|c|c|}
\hline entry & $\begin{array}{l}\text { diazo transfer } \\
\text { reagent }\end{array}$ & $\begin{array}{l}\text { Yoshida } \\
\text { IS }\end{array}$ & $\begin{array}{l}\text { Yoshida } \\
\text { EP }\end{array}$ & $\begin{array}{l}\text { Pfizer-modified } \\
\text { IS }\end{array}$ & $\begin{array}{l}\text { Pfizer-modified } \\
\text { EP }\end{array}$ & $\mathrm{IS}^{a}$ & explosive $^{b}$ \\
\hline 1 & $\mathrm{TsN}_{3}$ & 0.20 & 0.19 & 0.56 & 0.37 & + & $+^{c}$ \\
\hline 2 & $\mathrm{TsN}_{3}$ & 0.07 & 0.10 & 0.53 & 0.34 & + & $+{ }^{c}$ \\
\hline 3 & $\mathrm{TsN}_{3}$ & -0.11 & -0.07 & 0.34 & 0.16 & + & $+{ }^{c}$ \\
\hline 4 & $\mathrm{MsN}_{3}$ & 0.33 & 0.32 & 0.69 & 0.51 & + & + \\
\hline 5 & $p$-DBSA & -0.27 & -0.25 & 0.11 & -0.05 & - & \\
\hline 6 & $p$-ABSA & -0.20 & -0.17 & 0.24 & 0.06 & $\sim d$ & \\
\hline 7 & $p$-CBSA & -0.13 & -0.08 & 0.30 & 0.14 & $\sim d$ & \\
\hline 8 & $m$-CBSA & -0.09 & -0.05 & 0.37 & 0.19 & & \\
\hline 9 & o-NBSA & 0.30 & 0.28 & 0.66 & 0.47 & + & + \\
\hline 10 & 18 & 0.09 & 0.10 & 0.46 & 0.30 & + & + \\
\hline 11 & 19 & 0.02 & 0.02 & 0.39 & 0.21 & + & \\
\hline 12 & 21 & -0.04 & -0.02 & 0.34 & 0.18 & + & \\
\hline 13 & 22 & -0.15 & -0.14 & 0.22 & 0.05 & - & \\
\hline 14 & 23 & -0.71 & -0.67 & -0.25 & -0.43 & - & \\
\hline 15 & $\mathrm{NfN}_{3}$ & -0.47 & -0.45 & -0.05 & -0.23 & - & - \\
\hline 16 & ADT & -0.14 & -0.08 & 0.31 & 0.16 & - & \\
\hline
\end{tabular}

a Classification defined by the borderline sensitive compound $m$-dinitrobenzene ( $m$-DNB) which has an IS of $39 \mathrm{~J} .{ }^{62}$ It is reported that the impact sensitivity observed in some sulfonyl azides is due to a small amount of impurity, which can derive from their synthesis in alcohols rather than acetone/water. ${ }^{63,64}{ }^{b}$ No statement can be made as to the explosivity of a substance if no data is available. ${ }^{c}$ The only literature report of the explosivity of $\mathrm{TsN}_{3}$ is from a private communication. ${ }^{65}$ d Classified as 'less sensitive'. 
S. P. Green, K. M. Wheelhouse, A. D. Payne, J. P. Hallett, P. W. Miller and J. A. Bull

\section{Table S3. Average Yoshida IS and EP Correlations for all Diazo Compound DSC Data}

The complete Yoshida correlation output for each average diazo compound result from Table 3. Values $>0$ (in red) indicate a positive prediction of the property; values $<0$ indicate a negative prediction.

\begin{tabular}{|c|c|c|c|c|c|}
\hline entry & compound $^{a}$ & Yoshida IS & Yoshida EP & Pfizer-modified IS & Pfizer-modified EP \\
\hline 1 & 9 & -0.26 & -0.29 & 0.16 & -0.07 \\
\hline 2 & $9^{b}$ & -0.23 & -0.27 & 0.21 & -0.04 \\
\hline 3 & 10 & -0.23 & -0.26 & 0.20 & -0.04 \\
\hline 4 & $10^{b}$ & -0.20 & -0.26 & 0.20 & -0.04 \\
\hline 5 & 34 & -0.30 & -0.34 & 0.16 & -0.10 \\
\hline 6 & 35 & -0.47 & -0.50 & -0.07 & -0.29 \\
\hline 7 & 36 & -0.43 & -0.47 & -0.01 & -0.25 \\
\hline 8 & 37 & -0.36 & -0.41 & -0.02 & -0.23 \\
\hline 9 & 38 & -0.19 & -0.29 & 0.22 & -0.07 \\
\hline 10 & 39 & -0.28 & -0.32 & 0.15 & -0.10 \\
\hline 11 & 40 & -0.26 & -0.31 & 0.20 & -0.07 \\
\hline 12 & 41 & -0.36 & -0.38 & 0.05 & -0.17 \\
\hline 13 & 42 & -0.43 & -0.45 & -0.03 & -0.24 \\
\hline 14 & 43 & -0.41 & -0.43 & 0.02 & -0.20 \\
\hline 15 & 44 & -0.30 & -0.30 & 0.11 & -0.08 \\
\hline 16 & 45 & -0.32 & -0.35 & 0.11 & -0.13 \\
\hline 17 & 46 & -0.30 & -0.34 & 0.14 & -0.11 \\
\hline 18 & 47 & -0.36 & -0.38 & 0.08 & -0.15 \\
\hline 19 & 48 & -0.35 & -0.37 & 0.07 & -0.15 \\
\hline 20 & 49 & -0.42 & -0.44 & 0.01 & -0.22 \\
\hline 21 & 50 & -0.16 & -0.25 & 0.28 & -0.02 \\
\hline 22 & $50^{b}$ & -0.18 & -0.29 & 0.23 & -0.07 \\
\hline 23 & 51 & -0.24 & -0.30 & 0.20 & -0.07 \\
\hline 24 & 52 & -0.26 & -0.32 & 0.17 & -0.10 \\
\hline 25 & 53 & -0.36 & -0.40 & 0.07 & -0.17 \\
\hline 26 & 54 & -0.44 & -0.46 & -0.03 & -0.25 \\
\hline 27 & 55 & -0.29 & -0.34 & 0.12 & -0.12 \\
\hline 28 & 56 & -0.24 & -0.32 & 0.14 & -0.12 \\
\hline 29 & 57 & -0.43 & -0.49 & 0.00 & -0.26 \\
\hline 30 & $57^{b}$ & -0.55 & -0.63 & -0.13 & -0.40 \\
\hline 31 & 58 & -0.34 & -0.41 & -0.01 & -0.23 \\
\hline 32 & 59 & -0.38 & -0.42 & -0.04 & -0.24 \\
\hline 33 & 60 & -0.43 & -0.43 & -0.06 & -0.24 \\
\hline 34 & 61 & -0.29 & -0.33 & 0.12 & -0.11 \\
\hline 35 & 62 & -0.34 & -0.36 & 0.06 & -0.16 \\
\hline 36 & 63 & -0.52 & -0.43 & -0.09 & -0.20 \\
\hline 37 & EDA & 0.10 & 0.04 & 0.41 & 0.22 \\
\hline 38 & 64 & -0.02 & -0.11 & -0.30 & 0.06 \\
\hline 39 & 65 & -0.30 & -0.32 & 0.15 & -0.08 \\
\hline 40 & 66 & -0.22 & -0.22 & 0.26 & 0.03 \\
\hline 41 & $66^{b}$ & -0.24 & -0.23 & 0.22 & 0.01 \\
\hline 42 & 67 & -0.39 & -0.39 & 0.02 & -0.17 \\
\hline 43 & 68 & -0.46 & -0.43 & -0.01 & -0.19 \\
\hline 44 & 69 & -0.56 & -0.52 & -0.12 & -0.29 \\
\hline 45 & 70 & -0.28 & -0.33 & 0.11 & -0.12 \\
\hline 46 & 71 & -0.31 & -0.33 & 0.09 & -0.12 \\
\hline 47 & 72 & -0.41 & -0.46 & 0.24 & -0.12 \\
\hline 48 & 73 & -0.25 & -0.30 & 0.20 & -0.07 \\
\hline 49 & 74 & -0.36 & -0.42 & 0.12 & -0.17 \\
\hline
\end{tabular}

${ }^{a}$ Results using DSC method A unless otherwise noted. ${ }^{b}$ Results using DSC method B. 
S. P. Green, K. M. Wheelhouse, A. D. Payne, J. P. Hallett, P. W. Miller and J. A. Bull

Figure S104. Plot of temperature and pressure during the ARC experiment with ADT.

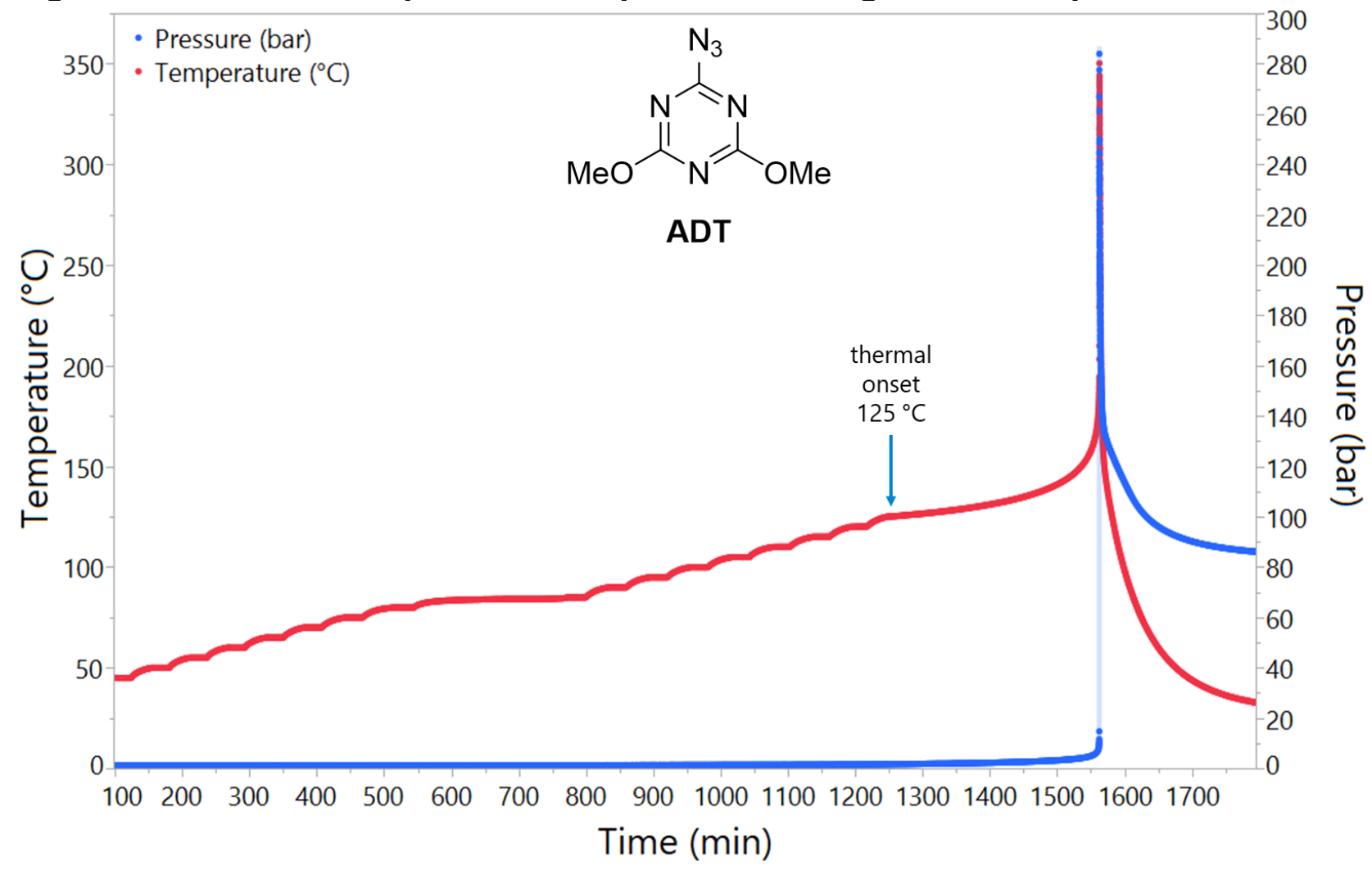




\section{Table S4. Impact Sensitivity of Well-Defined Explosives ${ }^{66}$}

The categories for impact sensitivity are as follows, as defined by Klapötke:67

- very sensitive: $\leq 3 \mathrm{~J}$

- sensitive: $3-4 \mathrm{~J}$

- less sensitive: $4-40 \mathrm{~J}$

- insensitive $>50 \mathrm{~J}$

This following are impact sensitivity results given for well-known compounds to give a comparison to the impact sensitivity results presented for the diazo transfer reagents in Table 2 of the manuscript.

\begin{tabular}{llr}
\hline entry & compound & Impact Sensitivity $(\mathrm{J})$ \\
\hline 1 & nitroglycerine (NG) & 0.2 \\
2 & lead azide & $2.4-7.5$ \\
3 & RDX & 7.5 \\
4 & TNT & 15 \\
5 & m-DNB & 39 \\
6 & ammonium nitrate & $>50$ \\
\hline
\end{tabular}


S. P. Green, K. M. Wheelhouse, A. D. Payne, J. P. Hallett, P. W. Miller and J. A. Bull

\section{${ }^{1} \mathrm{H}$ and ${ }^{13} \mathrm{C}$ NMR Spectra of Novel Compounds:}

Ethyl (3-trifluoromethylphenyl)diazoacetate (49)

${ }^{1} \mathrm{H} \mathrm{NMR}\left(400 \mathrm{MHz}, \mathrm{CDCl}_{3}\right)$

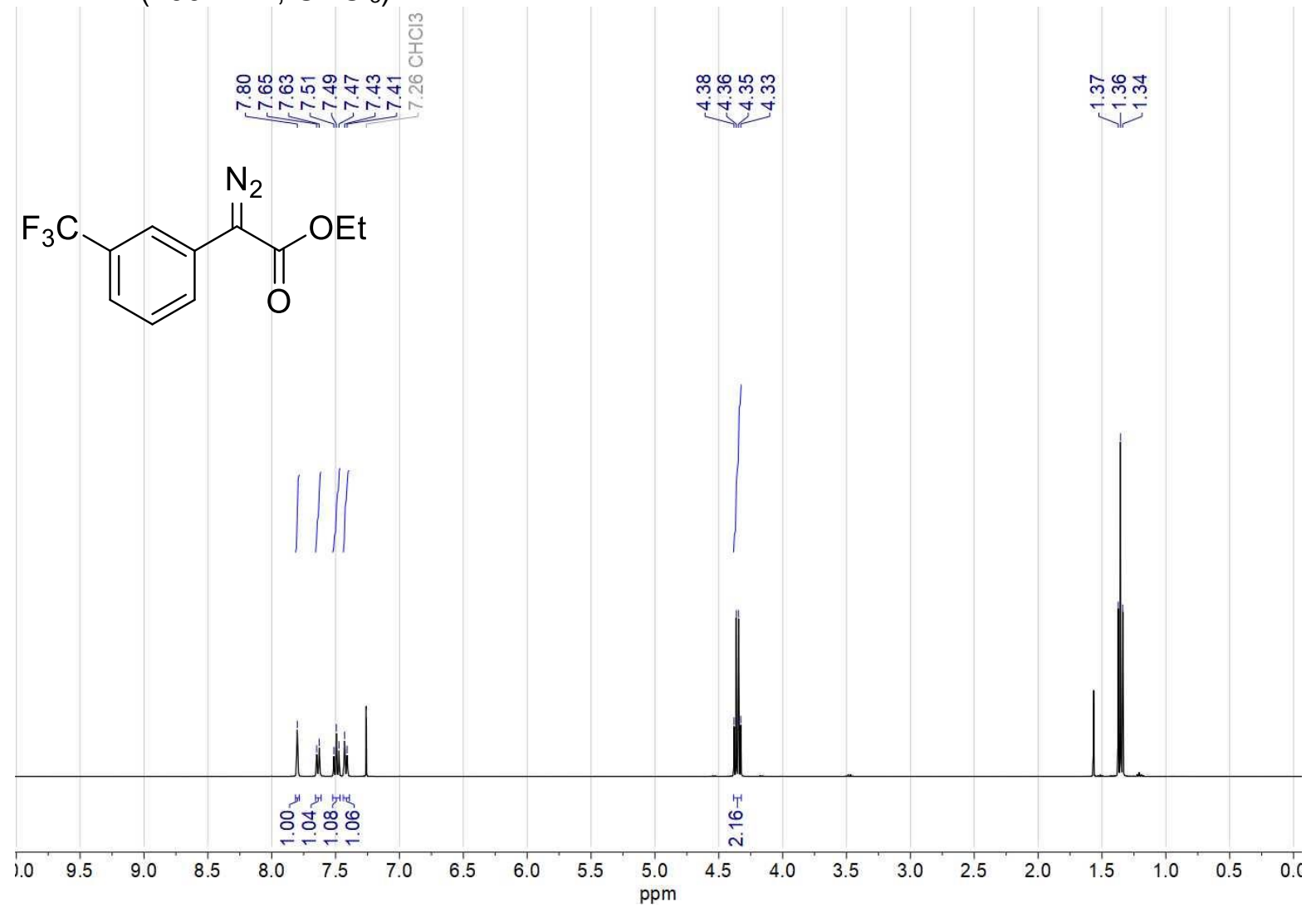

${ }^{13} \mathrm{C} \mathrm{NMR}\left(101 \mathrm{MHz}, \mathrm{CDCl}_{3}\right)$
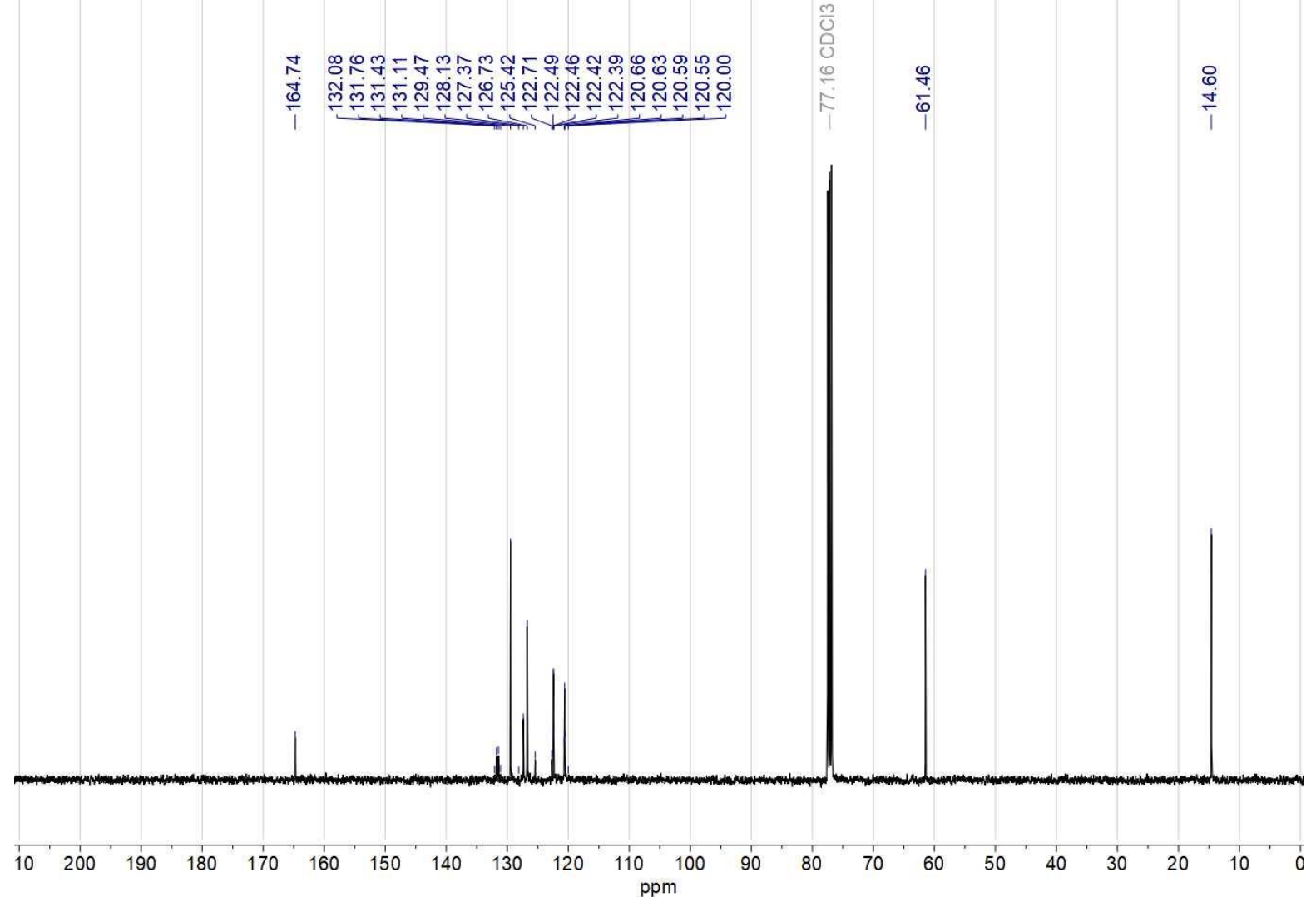


\section{Ethyl (4-phenoxyphenyl)diazoacetate (58)}

${ }^{1} \mathrm{H}$ NMR $\left(400 \mathrm{MHz}, \mathrm{CDCl}_{3}\right)$

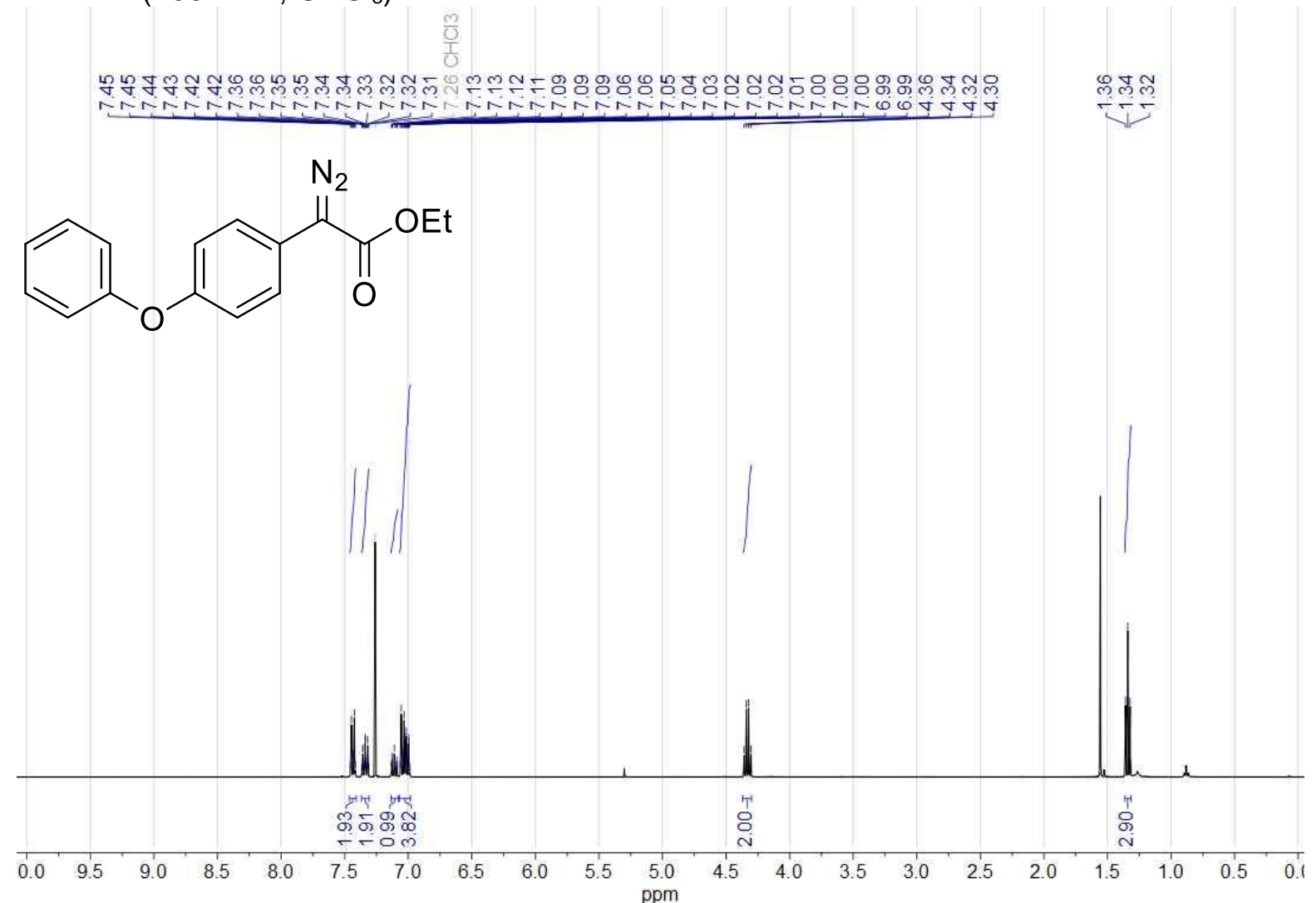

${ }^{13} \mathrm{C} \mathrm{NMR}\left(101 \mathrm{MHz}, \mathrm{CDCl}_{3}\right)$

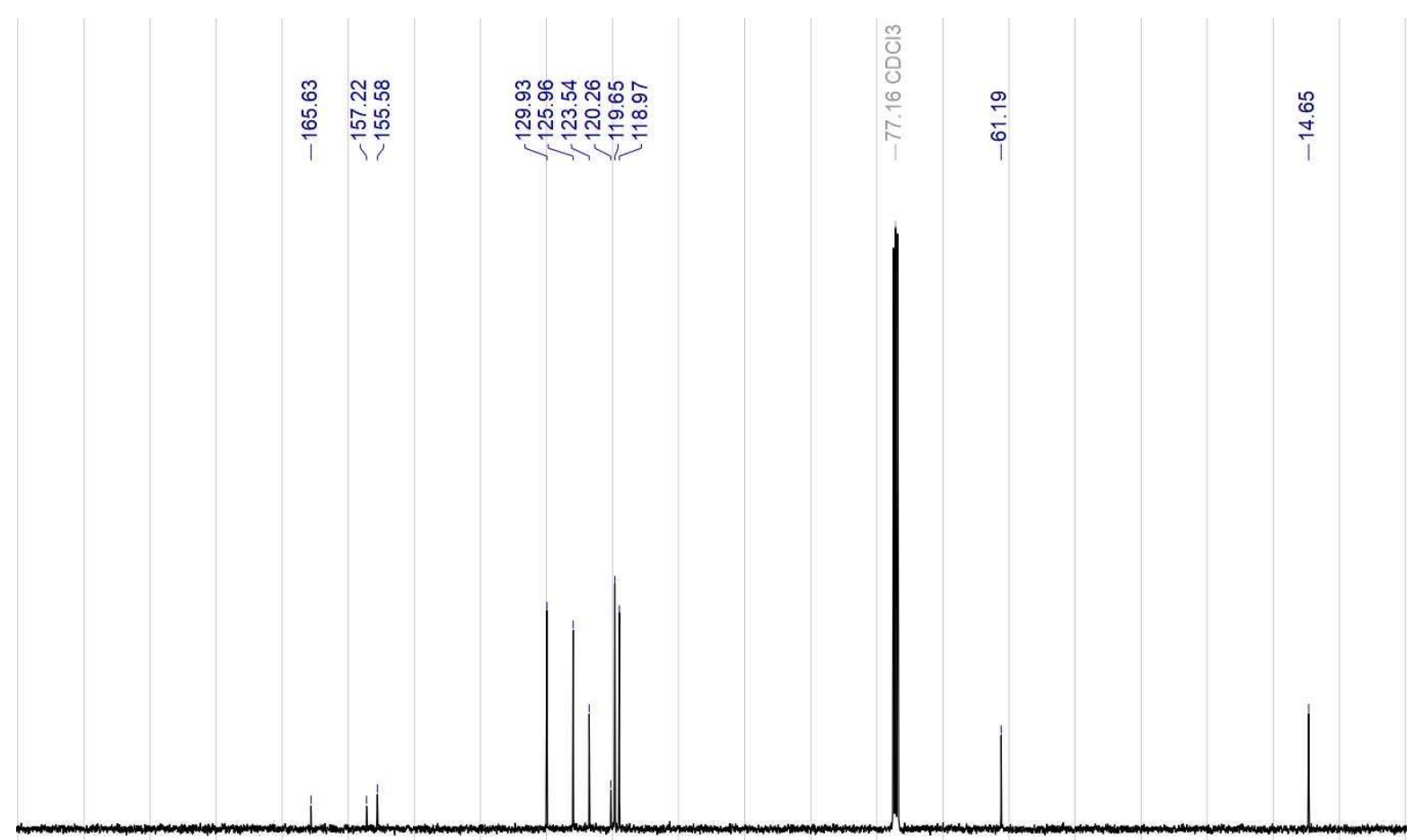




\section{References}

(1) Lee, S.; Beare, N. A.; Hartwig, J. F. Palladium-Catalyzed a-Arylation of Esters and Protected Amino Acids. J. Am. Chem. Soc. 2001, 123, 8410-8411.

(2) Pramanik, S.; Reddy, R. R.; Ghorai, P. Intramolecular Rearrangement of a-Azidoperoxides: An Efficient Synthesis of tert-Butyl Esters. Org. Lett. 2015, 17, 1393-1396.

(3) Periasamy, M.; Subramaniapillai, S. G.; Suresh, S. TiCl 4 Promoted Menthyl Ester Chiral Auxiliary Mediated Synthesis of Chiral Syn- $\beta$-Amino Esters and Applications of a Representative Syn- $\beta$-Amino Ester. Tetrahedron Asymmetry 2010, 21, 385-392.

(4) Takeda, K.; Tsuboyama, K.; Takayanagi, H.; Ogura, H. S,S'-Bis(1-phenyl-1H-tetrazol-5-yl) Dithiocarbonate: A New Esterification Reagent. Synthesis 1987, 560-562.

(5) Lee, J. I. A Novel Synthesis of Carboxylic Esters from 2-Methyl-4,6-pyrimidyl Dicarbonates and Grignard Reagents. Bull. Korean Chem. Soc. 2011, 32, 1765-1768.

(6) Katritzky, A. R.; Zhang, S.; Haleem, A.; Hussein, M.; Fang, Y.; Steel, P. J. One-Carbon Homologation of Carboxylic Acids via BtCH${ }_{2}$ TMS: A Safe Alternative to the Arndt-Eistert Reaction. J. Org. Chem. 2001, 66 , 5606-5612.

(7) Ke, J.; He, C.; Liu, H.; Xu, H.; Lei, A. Alcohol Assisted C-C Bond Breaking: Copper-Catalyzed Deacetylative $\alpha$-Arylation of $\beta$-Keto Esters and Amides. Chem. Commun. 2013, 49, 6767-6769.

(8) Ríos-Lombardía, N.; Busto, E.; García-Urdiales, E.; Gotor-Fernández, V.; Gotor, V. Enzymatic Desymmetrization of Prochiral 2-Substituted-1,3-Diamines: Preparation of Valuable Nitrogenated Compounds. J. Org. Chem. 2009, 74, 2571-2574.

(9) Best, D.; Jean, M.; Van De Weghe, P. Modular Synthesis of Arylacetic Acid Esters, Thioesters, and Amides from Aryl Ethers via Rh(II)-Catalyzed Diazo Arylation. J. Org. Chem. 2016, 81, 7760-7770.

(10) Artico, M.; Silvestri, R.; Stefancich, G.; Avigliano, L.; Di Giulio, A.; Maccarrone, M.; Agostinelli, E.; Mondovi, B.; Morpurgo, L. Aromatic Hydrazides as Specific Inhibitors of Bovine Serum Amine Oxidase. Eur. J. Med. Chem. 1992, 27, 219-228.

(11) Wu, G.; Deng, Y.; Wu, C.; Zhang, Y.; Wang, J. Synthesis of a-Aryl Esters and Nitriles: Deaminative Coupling of a-Aminoesters and a-Aminoacetonitriles with Arylboronic Acids. Angew. Chem. Int. Ed. 2014, 53, 10510-10514.

(12) Emer, E.; Twilton, J.; Tredwell, M.; Calderwood, S.; Collier, T. L.; Liégault, B.; Taillefer, M.; Gouverneur, V. Diversity-Oriented Approach to $\mathrm{CF}_{3} \mathrm{CHF}-, \mathrm{CF}_{3} \mathrm{CFBr}_{-}, \mathrm{CF}_{3} \mathrm{CF}_{2^{-}},\left(\mathrm{CF}_{3}\right)_{2} \mathrm{CH}$-, and $\mathrm{CF}_{3}\left(\mathrm{SCF}_{3}\right) \mathrm{CH}$-Substituted Arenes from 1-(Diazo-2,2,2-trifluoroethyl)arenes. Org. Lett. 2014, 16, 6004-6007.

(13) Davis, O. A.; Bull, J. A. Synthesis of Di-, Tri-, and Tetrasubstituted Oxetanes by Rhodium-Catalyzed $\mathrm{O}-\mathrm{H}$ Insertion and C-C Bond-Forming Cyclization. Angew. Chem. Int. Ed. 2014, 53, 14230-14234.

(14) Davis, O. A.; Croft, R. A.; Bull, J. A. Synthesis of Diversely Functionalised 2,2-Disubstituted Oxetanes: Fragment Motifs in New Chemical Space. Chem. Commun. 2015, 51, 15446-15449.

(15) Boddy, A. J.; Affron, D. P.; Cordier, C. J.; Rivers, E. L.; Spivey, A. C.; Bull, J. A. Rapid Assembly of Saturated Nitrogen Heterocycles in One-Pot: Diazo-Heterocycle "Stitching" by N-H Insertion and Cyclization. Angew. Chem. Int. Ed. 2019, 58, 1458-1462.

(16) Boddy, A. J.; Cordier, C. J.; Goldberg, K.; Madin, A.; Spivey, A. C.; Bull, J. A. Acid-Mediated Ring Expansion of 2,2-Disubstituted Azetidine Carbamates to 6,6-Disubstituted 1,3-Oxazinan-2-ones. Org. Lett. 2019, 21, 1818-1822.

(17) Padwa, A.; Sá, M. M.; Weingarten, M. D. Metal Substituted Diazo Esters as Substrates for Cross Coupling Reactions. Tetrahedron 1997, 53, 2371-2386.

(18) Davies, H. M. L.; Grazini, M. V. A.; Aouad, E. Asymmetric Intramolecular C-H Insertions of Aryldiazoacetates. Org. Lett. 2001, 3, 1475-1477.

(19) Gao, L.; Hwang, G. S.; Ryu, D. H. Oxazaborolidinium lon-Catalyzed Cyclopropanation of aSubstituted Acroleins: Enantioselective Synthesis of Cyclopropanes Bearing Two Chiral Quaternary Centers. J. Am. Chem. Soc. 2011, 133, 20708-20711.

(20) Hashimoto, T.; Naganawa, Y.; Kano, T.; Maruoka, K. Construction of Stereodefined 1,1,2,2Tetrasubstituted Cyclopropanes by Acid Catalyzed Reaction of Aryldiazoacetates and $\alpha$-Substituted Acroleins. Chem. Commun. 2007, 5143-5145.

(21) Peng, C.; Cheng, J.; Wang, J. Palladium-Catalyzed Cross-Coupling of Aryl or Vinyl lodides with Ethyl Diazoacetate. J. Am. Chem. Soc. 2007, 129, 8708-8709. 
(22) Luo, X.; Chen, G.; He, L.; Huang, X. Amination of Diazocarbonyl Compounds: N-H Insertion under Metal-Free Conditions. J. Org. Chem. 2016, 81, 2943-2949.

(23) Hahn, N. D.; Nieger, M.; Dötz, K. H. Efficient and Regioselective Chromium(0)-Catalyzed Reaction of 2-Substituted Furans with Diazo Compounds: Stereoselective Synthesis of (2E,4Z)-2-Arylhexadienedioic Acid Diesters. J. Organomet. Chem. 2004, 689, 2662-2673.

Ye, F.; Wang, C.; Zhang, Y.; Wang, J. Synthesis of Aryldiazoacetates through Palladium(0)Catalyzed Deacylative Cross-Coupling of Aryl lodides with Acyldiazoacetates. Angew. Chem. Int. Ed. 2014, 53, 11625-11628.

(25) Ye, F.; Qu, S.; Zhou, L.; Peng, C.; Wang, C.; Cheng, J.; Hossain, M. L.; Liu, Y.; Zhang, Y.; Wang, Z. X.; Wang, J. Palladium-Catalyzed C-H Functionalization of Acyldiazomethane and Tandem CrossCoupling Reactions. J. Am. Chem. Soc. 2015, 137, 4435-4444.

Hu, M.; Ni, C.; Hu, J. Copper-Mediated Trifluoromethylation of $\alpha$-Diazo Esters with TMSCF$_{3}$ : The Important Role of Water as a Promoter. J. Am. Chem. Soc. 2012, 134, 15257-15260.

(27) Zheng, J.; Lin, J. H.; Yu, L. Y.; Wei, Y.; Zheng, X.; Xiao, J. C. Cross-Coupling between Difluorocarbene and Carbene-Derived Intermediates Generated from Diazocompounds for the Synthesis of Gem-Difluoroolefins. Org. Lett. 2015, 17, 6150-6153.

(28) Doyle, K. J.; Moody, C. J. The Rhodium Carbenoid Route to Oxazoles. Synthesis of 4-Functionalised Oxazoles; Three Step Preparation of a Bis-Oxazole. Tetrahedron 1994, 50, 3761-3772.

(29) Lee, J. C.; Yuk, J. Y. An Improved and Efficient Method for Diazo Transfer Reaction of Active Methylene Compounds. Synth. Commun. 1995, 25, 1511-1515.

(30) Baum, J. S.; Shook, D. A.; Davies, H. M. L.; Smith, H. D. Diazotransfer Reactions with p-Acetamidobenzenesulfonyl Azide. Synth. Commun. 1987, 17, 1709-1716.

Marcoux, D.; Azzi, S.; Charette, A. B. TfNH 2 as Achiral Hydrogen-Bond Donor Additive to Enhance the Selectivity of a Transition Metal Catalyzed Reaction. Highly Enantio- And Diastereoselective Rhodium-Catalyzed Cyclopropanation of Alkenes Using a-Cyano Diazoacetamide. J. Am. Chem. Soc. 2009, 131, 6970-6972.

(32) Busman, S. C.; Cuny, G. D.; Zaklika, K. A.; Ellis, R. J. Diazo Compounds for Laser-Induced Mass Transfer Imaging Materials. US Patent 1998/5756689 A1, 1998.

(33) Green, G. M.; Peet, N. P.; Metz, W. A. Polystyrene-Supported Benzenesulfonyl Azide: A Diazo Transfer Reagent That Is Both Efficient and Safe. J. Org. Chem. 2001, 66, 2509-2511.

(34) Khare, A. B.; McKenna, C. E. An Improved Synthesis of Tetraalkyl Diazomethylenediphosphonates and Alkyl Diazo(dialkoxyphosphoryl)acetates. Synthesis 1991, 405-406.

(35) Wurz, R. P.; Lin, W.; Charette, A. B. Trifluoromethanesulfonyl Azide: An Efficient Reagent for the Preparation of [Alpha]-cyano-[alpha]-diazo Carbonyls and an [Alpha]-sulfonyl-[alpha]-diazo Carbonyl. Tetrahedron Lett. 2003, 44, 8845-8848.

(36) Ciganek, E. a-Aryl- and a-Cyanodiazoacetic Esters. J. Org. Chem. 1970, 35, 862-864.

(37) Keipour, H.; Jalba, A.; Delage-Laurin, L.; Ollevier, T. Copper-Catalyzed Carbenoid Insertion Reactions of $\alpha$-Diazoesters and $\alpha$-Diazoketones into Si-H and S-H Bonds. J. Org. Chem. 2017, 82, 3000-3010.

(38) Davies, H. M. L. L.; Hansen, T.; Churchill, M. R. Catalytic Asymmetric C-H Activation of Alkanes and Tetrahydrofuran. J. Am. Chem. Soc. 2000, 122, 3063-3070.

(39) Aller, E.; Brown, D. S.; Cox, G. G.; Miller, D. J.; Moody, C. J. Diastereoselectivity in the O-H Insertion Reactions of Rhodium Carbenoids Derived from Phenyldiazoacetates of Chiral Alcohols. Preparation of $\alpha$-Hydroxy and a-Alkoxy Esters. J. Org. Chem. 1995, 60, 4449-4460.

(40) Tortoreto, C.; Rackl, D.; Davies, H. M. L. Metal-Free C-H Functionalization of Alkanes by Aryldiazoacetates. Org. Lett. 2017, 19, 770-773.

(41) Creary, X.; Mehrsheikh-Mohammadi, M. E. Captodative Rate Enhancements in the Methylenecyclopropane Rearrangement. J. Org. Chem. 1986, 51, 2664-2668.

Lefebvre, Q.; Fava, E.; Nikolaienko, P.; Rueping, M. Hydrotrifluoromethylthiolation of a-Diazo Esters Synthesis of $\alpha-\mathrm{SCF}_{3}$ Substituted Esters. Chem. Commun. 2014, 50, 6617-6619.

Nicolle, S. M.; Moody, C. J. Potassium N-lodo $p$-Toluenesulfonamide (TsNIK, lodamine-T): A New Reagent for the Oxidation of Hydrazones to Diazo Compounds. Chem. Eur. J. 2014, 20, 4420-4425. Fu, L.; Wang, H.; Davies, H. M. L. Role of Ortho -Substituents on Rhodium-Catalyzed Asymmetric Synthesis of $\beta$-Lactones by Intramolecular $\mathrm{C}-\mathrm{H}$ Insertions of Aryldiazoacetates. Org. Lett. 2014, 16, 

3036-3039.

(45) Zha, G. F.; Han, J. Bin; Hu, X. Q.; Qin, H. L.; Fang, W. Y.; Zhang, C. P. Silver-Mediated Direct Trifluoromethoxylation of $\alpha$-Diazo Esters: Via the ${ }^{-} \mathrm{OCF}_{3}$ Anion. Chem. Commun. 2016, 52, 74587461.

(46) Santos, F. M. F.; Rosa, J. N.; André, V.; Duarte, M. T.; Veiros, L. F.; Gois, P. M. P. N -Heterocyclic Carbene Catalyzed Addition of Aldehydes to Diazo Compounds: Stereoselective Synthesis of N-Acylhydrazones. Org. Lett. 2013, 15, 1760-1763.

(47) Chiara, J. L.; Suárez, J. R. Synthesis of a-Diazo Carbonyl Compounds with the Shelf-Stable Diazo Transfer Reagent Nonafluorobutanesulfonyl Azide. Adv. Synth. Catal. 2011, 353, 575-579. O'Bannon, P. E.; Dailey, W. P. Nitrocyclopropanes from Nitrodiazomethanes. Preparation and Reactivity. Tetrahedron 1990, 46, 7341-7358.

(49) Charette, A. B.; Wurz, R. P.; Ollevier, T. Trifluoromethanesulfonyl Azide: A Poweful Reagent for the Preparation of $\alpha$-Nitro- $\alpha$-diazocarbonyl Derivatives. J. Org. Chem. 2000, 65, 9252-9254.

(50) Denton, J. R.; Sukumaran, D.; Davies, H. M. L. Enantioselective Synthesis of TrifluoromethylSubstituted Cyclopropanes. Org. Lett. 2007, 9, 2625-2628.

(51) Bélanger, D.; Tong, X.; Soumaré, S.; Dory, Y. L.; Zhao, Y. Cyclic Peptide-Polymer Complexes and Their Self-Assembly. Chem. Eur. J. 2009, 15, 4428-4436.

(52) O'Mahony, R. M.; Broderick, C. M.; Lynch, D.; Collins, S. G.; Maguire, A. R. Synthesis and Use of a Cost-Effective, Aqueous Soluble Diazo Transfer Reagent $-m$-Carboxybenzenesulfonyl Azide.

Tetrahedron Lett. 2018, 60, 35-39.

(53) Brodsky, B. H.; du Bois, J. Deacylative Oxidation Strategy for the Preparation of R-Functionalized Carbonyls. Org. Lett. 2004, 6, 2619-2621.

(54) Zhu, S.-Z. Synthesis and Reactions of Fluoroalkanesulfonyl Azides and N,NDichlorofluoroalkanesulfonamides. J. Chem. Soc. Perkin Trans. 11994, 113, 2077-2081.

(55) Green, S. P.; Payne, A. D.; Wheelhouse, K. M.; Hallett, J. P.; Miller, P. W.; Bull, J. A. Diazo-Transfer Reagent 2-Azido-4,6-Dimethoxy-1,3,5-Triazine Displays Highly Exothermic Decomposition Comparable to Tosyl Azide. J. Org. Chem. 2019, 84, 5893-5898.

(56) Xie, S.; Yan, Z.; Li, Y.; Song, Q.; Ma, M. Intrinsically Safe and Shelf-Stable Diazo-Transfer Reagent for Fast Synthesis of Diazo Compounds. J. Org. Chem. 2018, 83, 10916-10921.

(57) Hendrickson, J. B.; Wolf, W. A. The Direct Introduction of the Diazo Function in Organic Synthesis. J. Org. Chem. 1968, 33, 3610-3618.

(58) Raz, R.; Rademann, J. Fmoc-Based Synthesis of Peptide Thioacids for Azide Ligations via 2Cyanoethyl Thioesters. Org. Lett. 2012, 14, 5038-5041.

(59) Moreau, R. J.; Sorensen, E. J. Classical Carbonyl Reactivity Enables a Short Synthesis of the Core Structure of Acutumine. Tetrahedron 2007, 63, 6446-6453.

(60) Budinská, A.; Václavík, J.; Matoušek, V.; Beier, P. Nucleophilic Tetrafluoroethylation Employing in Situ Formed Organomagnesium Reagents. Org. Lett. 2016, 18, 5844-5847.

(61) Hansch, C.; Leo, A.; Taft, R. W. A Survey of Hammett Substituent Constants and Resonance and Field Parameters. Chem. Rev. 1991, 91, 165-195.

(62) Yoshida, T.; Yoshizawa, F.; Itoh, M.; Matsunaga, T.; Watanabe, M. Prediction of Fire and Explosion Hazard for Reactive Chemicals (I): Estimation of Explosive Properties of Self-Reactive Chemicals from SC-DSC Data. Kogyo Kayak. 1987, 48, 311-316.

(63) Breslow, D. S.; Sloan, M. F.; Newburg, N. R.; Renfrow, W. B. Thermal Reactions of Sulfonyl Azides. J. Am. Chem. Soc. 1969, 91, 2273-2279.

(64) Curphey, T. J. Preparation of $p$-Toluenesulfonyl Azide. A Cautionary Note. Org. Prep. Proced. Int. 1981, 13, 112-115.

(65) Hazen, G. G.; Weinstock, L. M.; Connell, R.; Bollinger, F. W. A Safer Diazotransfer Reagent. Synth. Commun. 1981, 11, 947-956.

(66) Meyer, R.; Köhler, J.; Homburg, A. Explosives, 6th ed.; Wiley-VCH Verlag GmbH: Weinheim, Germany, 2007.

(67) Fischer, N.; Goddard-Borger, E. D.; Greiner, R.; Klapötke, T. M.; Skelton, B. W.; Stierstorfer, J. Sensitivities of Some Imidazole-1-sulfonyl Azide Salts. J. Org. Chem. 2012, 77, 1760-1764. 